Frédéric Döhl

Musikgeschichte

ohne Markennamen

Soziologie und Ästhetik des Klavierquintetts
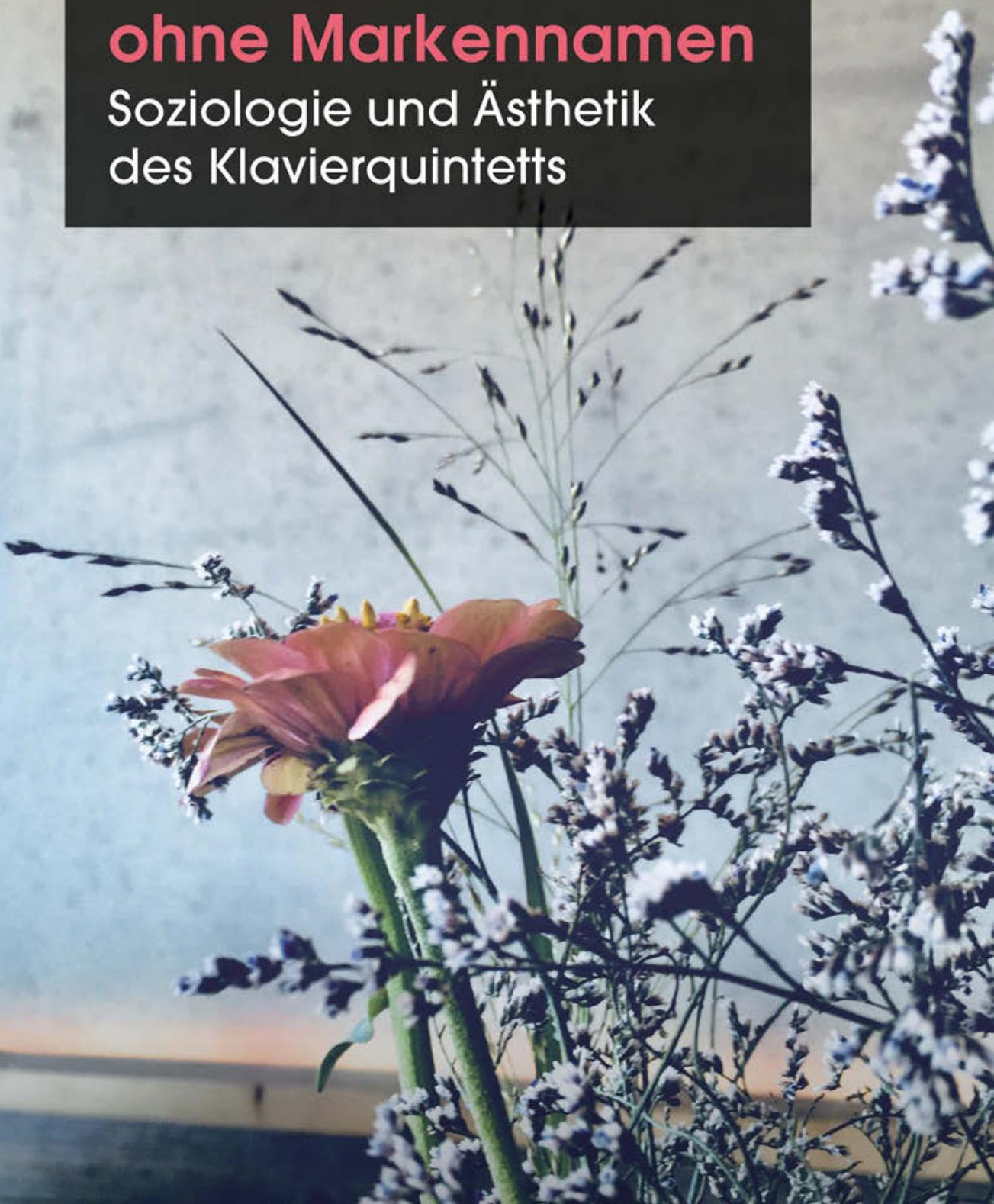

[transcript] Musik und Klangkuifur 
Frédéric Döhl

Musikgeschichte ohne Markennamen

Musik und Klangkultur | Band 26 
Für Bruno

Frédéric Döhl (PD Dr. phil. Ass. iur.), geb. 1978, ist Strategiereferent für Digital Humanities der Deutschen Nationalbibliothek und lehrt Musikwissenschaft und Musikjournalismus an der Technischen Universität Dortmund und der Freien Universität Berlin. Seine Arbeitsschwerpunkte sind Adaption Studies, Genretheorie und -geschichte, Digital Humanities, Musikjournalismus/-vermittlung sowie Urheber- und Medienrecht. 
FRÉDÉRIC DÖHL

\section{Musikgeschichte ohne Markennamen}

Soziologie und Ästhetik des Klavierquintetts

[transcript] 
Die frei zugängliche digitale Publikation wurde ermöglicht mit Mitteln des BMBF-Projektes OGeSoMo der Universitätsbibliothek Duisburg-Essen. In diesem Projekt wird Open Access für geistes- und sozialwissenschaftliche Monografien gefördert und untersucht. Informationen und Ergebnisse finden Sie unter https://www.uni-due.de/ogesomo.

\section{Bibliografische Information der Deutschen Nationalbibliothek}

Die Deutsche Nationalbibliothek verzeichnet diese Publikation in der Deutschen Nationalbibliografie; detaillierte bibliografische Daten sind im Internet über http://dnb.d-nb.de abrufbar.

\section{(c) $\underset{\mathrm{BY}}{(\mathrm{ND}}$}

Dieses Werk ist lizenziert unter der Creative Commons Attribution-NoDerivatives 4.0 Lizenz (BY-ND). Diese Lizenz erlaubt unter Voraussetzung der Namensnennung des Urhebers die Vervielfältigung und Verbreitung des Materials in jedem Format oder Medium für beliebige Zwecke, auch kommerziell, gestattet aber keine Bearbeitung. (Lizenztext: https://creativecommons.org/licenses/by-nd/4.o/deed.de)

Um Genehmigungen für Adaptionen, Übersetzungen oder Derivate einzuholen, wenden Sie sich bitte an rights@transcript-verlag.de

Die Bedingungen der Creative-Commons-Lizenz gelten nur für Originalmaterial. Die Wiederverwendung von Material aus anderen Quellen (gekennzeichnet mit Quellenangabe) wie z.B. Schaubilder, Abbildungen, Fotos und Textauszüge erfordert ggf. weitere Nutzungsgenehmigungen durch den jeweiligen Rechteinhaber.

\section{(C 2019 transcript Verlag, Bielefeld}

Umschlaggestaltung: Maria Arndt, Bielefeld

Umschlagabbildung: (C) 2018 Frédéric Döhl

Druck: Majuskel Medienproduktion GmbH, Wetzlar

Print-ISBN 978-3-8376-4183-7

PDF-ISBN 978-3-8394-4183-1

https://doi.org/10.14361/9783839441831

Gedruckt auf alterungsbeständigem Papier mit chlorfrei gebleichtem Zellstoff. Besuchen Sie uns im Internet: https://www.transcript-verlag.de Bitte fordern Sie unser Gesamtverzeichnis und andere Broschüren an unter: info@transcript-verlag.de 


\section{Inhalt}

\section{Vorwort |7}

Soziologie des Klavierquintetts:

Zur Spezifik eines Genres | 9

Exkurs I: What of That?

Zur Frage nach den Möglichkeiten und

Grenzen der Digital Humanities für die

Musikgeschichtsschreibung | 37

Ästhetik des Klavierquintetts:

Situatives Hören, Emotional Impact

und das Kuratieren alternativer

Musikgeschichte | 57

Situatives Hören als Perspektive | 57

Herleitung der Perspektive | 59

»Emotional Impact« als Fokus der Perspektive | 61

Bewusstes Hören, Hören mit Ziel | 70

Alternative Musikgeschichte |72

Mixtape: Eine imaginierte, virtuelle Ausstellung musikalischer Situationen $\mid 82$

\section{Exkurs II:}

Möglichkeiten und Strategien

des Sprechens über Musik | 89

Problemlage | 89

Vier Modi der Annäherung | 97

Annäherung über Vergleich, Zuordnung und Einflussanalyse | 97

Annäherung über die Beschreibung der Machart | 99

Annäherung über die Beschreibung des Ausdrucks | 100

Annäherung über die Beschreibung des Kontexts | 102 


\section{Emotional Impact:}

67 Situationen seit Schumann | 105

Die Ausstellung | 105

Verortungen: Annäherungen an ausgewählte Situationen | 114

Anmerkungen | 151 


\section{Vorwort}

Dieses Buch ist eine kleine Meditation über etwas, das mich schon lange beschäftigt: Die Rolle des eigenen ästhetischen Erlebens für das Schreiben von Musikgeschichte. Ich habe es verfasst, um mir einiger Sachen klar zu werden. Vielleicht nützt es anderen, es zu lesen, die mit ähnlichen Fragen ringen.

Es entstand im Rahmen meiner Aufgaben als Strategiereferent für Digital Humanities im Leitungsstab der Generaldirektion der Deutschen Nationalbibliothek und als wissenschaftlicher Mitarbeiter am Lehrstuhl für $\mathrm{Mu}-$ sikjournalismus am Institut für Musik und Musikwissenschaft der Technischen Universität Dortmund. Beide Aufgabengebiete haben deutliche Spuren in dem hinterlassen, was folgen wird. Ich danke insbesondere Thomas Becker, Thilo Braun, Mario Dunkel, Daniel Martin Feige, Alexander Gurdon, Ruprecht Langer, Peter Leinen, Elisabeth Niggemann, Holger Noltze, Klaus Oehl, Albrecht Riethmüller, Ute Schwens, Michael Stegemann, Ramon Voges und Björn Woll für die Ermöglichung der Durchführung dieses Projekts im Rahmen meiner Aufgaben bzw. die Diskussion einzelner Aspekte, die Eingang in dieses Buch gefunden haben. Sowie meinen Studierenden über die vergangenen Jahre, mit denen ich die Möglichkeiten des Sprechens über Musik erkundet und diskutiert habe. Teile des Manuskripts haben Mario Dunkel und Sonja Seidl vorab kommentiert, wofür ich mich herzlich bedanke.

Die Finanzierung der Druckkosten erfolgte über Leistungsmittel am Institut für Theaterwissenschaft der Freien Universität Berlin. Die Finanzierung der Open-Access-Mittel erfolgte aus dem BMBF-geförderten Projekt OGeSoMo zur Förderung von Open-Access-Publikationen in den Geistes- 
und Sozialwissenschaften. Für beides bedanke ich mich, insbesondere bei Kathrin Höhner und Natalie Leinweber.

Mein besonderer Dank gilt darüber hinaus meiner Frau Anke Myrrhe für ihre Unterstützung. Die Arbeit ist unserem Sohn Bruno in Liebe gewidmet, der täglich ein kompromissloses Plädoyer lebt für die maßgebliche Bedeutung von »emotional impact« als Zugang zu Musik und Musikgeschichte.

Berlin, im April 2019

Frédéric Döhl 


\section{Soziologie des Klavierquintetts: Zur Spezifik eines Genres}

Vor einem Vierteljahrhundert stolperte ich in der Musikabteilung der Lübecker Stadtbibliothek über eine Aufnahme von Gabriel Faurés Klavierquintett Nr. 2 c-Moll (1921). Eingespielt im Jahr 1985 gemeinsam mit Faurés Klavierquintett Nr. 1 d-Moll op. 89 (1906) durch das Quintetto Fauré di Roma und erschienen bei dem kleinen Schweizer Label Claves. Meine erste Begegnung mit diesem Genre.

Es war ein Zufallsfund. Ich weiß es nicht mehr. Aber ich vermute, dass es das Coverdesign war, das mich ermutigte, jene Fauré-CD auszuleihen. Es zeigt einen Ausschnitt aus einem späten Seerosenbild Claude Monets. Ich mochte Monet. Von Fauré kannte ich hingegen bis dato nur ein Stück. Es war seine Berceuse D-Dur op. 16 für Violine und Klavier. Auch selbst das zu kennen, war einem Zufall geschuldet. Während eines Schulpraktikums im traditionsreichen Lübecker Musikhaus Ernst Robert, das selbst schon lange Geschichte ist, verkaufte ich dort Tonträger. Faurés Berceuse befand sich auf einem Sampler, den man mir empfahl. Seine Eleganz und heitere Melancholie gefielen mir. Ich war 14. Und entdeckte sehr viel für mich neue Musik in dieser einen Woche. Meine Eltern waren sehr musikliebend. Klassik spielte immer eine wichtige Rolle dabei. Aber wenn, dann liefen bei uns Standards. Mozart. Beethoven. Chopin. Oder Wagner. Vertraute Markennamen der Klassischen Musik. Erste Reihe der Prominenz. Kernbestandteile des Kanons. Die kleinstädtische Bibliothek meines Heimatortes nahe Lübeck hatte ihre Stärken eher im Bereich Fünf Freunde, Drei Fragezeichen und Was ist Was. Da war nicht viel zu holen. Das Internet mit seiner heutigen unfassbaren Angebotsvielfalt von YouTube über Spotify bis SoundCloud gab es noch nicht. Jedenfalls in meinem bundes- 
deutschen Alltag. Die lokalen Konzertsäle in nahen Lübeck waren tatsächlich weit weg. Ohne Auto kam man dort nämlich abends nicht mehr weg, derart sportlich früh fuhr die letzte Regionalbahn gegen $22 \mathrm{Uhr}$ in Richtung Süden. Man ging gelegentlich zum Schleswig-Holstein Musik Festival. Aber das blieb ein saisonales Ereignis. Und zu Konzerten vor Ort. Im Ratzeburger Dom vor allem. Und es gab wohl NDR3, Vorläufer des heutigen NDR Kultur. Doch davon wusste ich nichts. Ich hörte mich stattdessen nach der Entdeckung der Lübecker Musikbibliothek einmal quer durch deren reiche Bestände. Einmal in der Woche ging es für Instrumentalunterricht in die Hansestadt. Und anschließend zum Tauschen von Musik.

Das Klavierquintett ist das Offensichtlichste, was mir als Interesse für ein ganzes Genre aus dieser Erkundungsphase blieb. Eine enorme Sammlung an Tonaufnahmen ist über die Jahre entstanden. Ein ganzes Regal voll. Seit langem dreistellig und immer noch anwachsend. Und ein korrespondierender Auswahlordner »Klavierquintette « auf meinem iPod. Ein oft gehörter Ordner. Von der Eigenart eben dieses Ordners möchte ich hier erzählen. Seiner Eigenart als kuratierter Musikgeschichte. Als persönlicher Musikgeschichtsschreibung mittels Auswahl und Zusammenstellung. Von ihm nehmen meine Beobachtungen ihren Ausgang. Er wird den Referenzpunkt bilden. In ihm ist versammelt, was sich mit Paul Guyers Begriff des »emotional impact « adressieren lässt. Dieser wird zentral werden für das Weitere. Für das, was mich hier umtreibt.

Für die Frage nämlich nach den Alternativen zu einer allein an Markennamen orientierten Konstruktion und Tradierung von Musikgeschichte, wie sie den Umgang der Leitmedien Klassischer Musik mit dem Klavierquintett prägt. Hilflos vor einem Genre stehend, das sich von wenigen Ausnahmen abgesehen mangels Markennamen den üblichen Erschließungsstrategien und Vermittlungsmechanismen entzieht, von der Heroen- über die Einflussbis zur Gattungsmusikgeschichtsschreibung.

Und darauf aufbauend für das Nachdenken über die Grenzen der Digital Humanities als Basis und Strategie für eine solche alternative Musikgeschichtsschreibung. An dieser Stelle hier nicht ein Nachdenken über die technischen und organisatorischen, rechtlichen und ethischen Grenzen. Sondern die inhaltlichen. Was werden der Aufbau und die Auswertung großer Datenmengen auf ein Genre wie das Klavierquintett hin historiographisch nicht leisten können? Was muss notwendige Ergänzung und produktives Korrektiv dazu bleiben, egal, wie gut sich die Möglichkeiten der Da- 
tensammlung, -vernetzung und -erschließung auch weiterentwickeln werden, an denen derzeit an anderer Stelle in den Digital Humanities gearbeitet wird? U.a. von mir selbst. Als längerfristig angelegtes Pilotprojekt zum Klavierquintett in Rahmen meiner Aufgaben als Strategiereferent für Digital Humanities an der Deutschen Nationalbibliothek.

Dies ist mein zweiter Beitrag, der in Flankierung meiner eigenen Projektarbeit in den Digital Humanities über die Grenzen dieser Form digitaler Geisteswissenschaft als Basis und Strategie für Musikgeschichtsschreibung reflektiert. ${ }^{1}$ Nicht als Kritik an den Digital Humanities. Oder gar als deren Zurückweisung. ${ }^{2}$ Sondern als Teil eines Diskurses, der nicht zuletzt auch in den Digital Humanities selbst zu führen ist und geführt wird. ${ }^{3} \mathrm{Um}$ ihr spezifisches Potential in den Griff zu bekommen. Um dieses möglichst gezielt für die passenden historiographischen Fragestellungen einzusetzen. Um überhaupt zu verstehen, wo es passt. Was diese Annäherung an Musikgeschichte kann. Was aber auch nicht. Bei einer dem Grundsatz nach performativen und ephemeren Kunst wie der Musik, die oberndrein einer weithin eigenen Semantik folgt.

In der ersten Studie stand ebenfalls die Auseinandersetzung mit einem Genre im Vordergrund. Gegenstand war dort eine Tradition populärer amerikanischer A-cappella-Musik namens Barbershop Harmony. Ein ähnlich speziell gelagertes Genre wie es das Klavierquintett für die Klassische Musik darstellt. Gezeichnet von allerlei Besonderheiten und Eigenarten. Abseits des musikalischen Mainstreams gelegen. Aber aufgrund seiner Spezifik besonders geeignet, sehr präzise historiographische Fragen zu stellen. An den Gegenstand. Aber mit ihm vor allem über ihn hinaus. So beobachtet mein auf die Barbershop Harmony bezogenes Langzeitprojekt die Entwicklung digitaler Archive. Und zeigt, dass die Akkumulation von Daten und die Verfeinerung der Möglichkeiten ihrer Auswertung nicht notwendig historiographische Streitstände aufzulösen vermögen. Im Fall der Barbershop Harmony ist das der für das Genre konstitutive Konflikt darüber, ob es sich bei dieser musikalischen Praxis um eine Historische Aufführungspraxis oder eine Invented Tradition handelt. ${ }^{4}$

Auch mein Fallbeispiel hier, das Klavierquintett, ist geprägt von Besonderheiten und Eigenarten. Das Klavierquintett ist in seiner über mehr als zwei Jahrhunderte gewachsenen musikalischen Vielfalt als Genre musikalisch zuvorderst durch seine Besetzung definiert: Klavier plus Streichquartett. Allein wissenschaftlich sind über 1000 Werke erfasst. ${ }^{5}$ Man stößt auf 
ein weites Spektrum an Erscheinungsformen. Von Ein- bis Fünfsätzigkeit, von kurzen, nur wenige Minuten langen Stücken bis zu monumentalen Opera an der Stundengrenze und darüber hinaus ist alles dabei. Ebenso an Stilen. Satztechniken. Ensemblebalancen. Musikalischen Milieus. Ausdrucksabsichten. Virtuositätsgraden. Zielgruppen. Doch die Besetzung ist fix: Sie hält das Genre zusammen.

Es handelt sich beim Klavierquintett also um ein Genre und damit um einen Genrebegriff, für den Züge besonders konstitutiv sind, die man im landläufigen akademischen Sprachgebrauch vielleicht an anderer Stelle alternativ unter Gattung besprochen sieht, gerade im Kontext Klassischer Musik. ${ }^{6}$ Und steht damit zugleich in Kontrast zu Genres, die eine starke Überschneidung zu Charakteristika aufweisen, die wiederum andernorts bevorzugt unter Begriffen wie Subkultur, ${ }^{7}$ Szene $^{8}$ oder Stil ${ }^{9}$ beschrieben werden. Nichtsdestotrotz spreche ich im Folgenden von Genre - und wenn von Gattung, dann nicht in Abgrenzung, sondern der Abwechslung halber und synonym hierzu gemeint -, weil Genre der tendenziell weiter gefasste Begriff ist. Der dabei besonders klar und konsequent in seinem Gebrauch soziale Aushandlungsfragen als konstituierend mit im Blick hat. Und diese sind für das Klavierquintett als Genre genauso prägend wie das Besetzungsgebot.

Versucht man, den Genrebegriff noch weiter einzugrenzen oder gar zu definieren, stößt man bei aller Alltäglichkeit des Sprechens in Genrebegriffen auf eine enorme Diversivität und Komplexität. Eine ausführliche Diskussion habe ich als Endnote beigefügt, denn sie ist nicht das eigentliche Anliegen hier. ${ }^{10}$ Letztlich zwingt einen die dort eingehend beschriebene Situation vor allem dazu, klar zu sagen, was man selbst im eigenen Forschungskontext mit dem Genrebegriff meint und warum man es nützlich findet, mit ihm zu arbeiten. Wenn ich hier nun also Genre sage, meine ich nicht nur eine Besetzung und die damit einhergehenden musikalischen Möglichkeiten. Sondern einen Cluster aus Orientierungen, Erwartungen und Konventionen. ${ }^{11}$ Ein solcher Cluster setzt sich, falls die Genreetablierung gelingt, als ein zur Identität fähiges Netzwerk aus Produktion, Zirkulation und Bedeutung durch. Der Produktion z.B. von Werken, Tonaufnahmen und Aufführungen. Der Zirkulation z.B. von Tonträgern, Noten und Diskursen. Aber eben auch der Bedeutung, die Akteure des Genres diesem zuweisen oder aus ihm ziehen. Zur sozialen Identifikation. Oder für ökonomischen oder pädagogischen Gewinn. Oder als intellektuelle Herausfor- 
derung. Oder für »emotional impact«, worum es mir hier gehen wird. Dieser Cluster integriert dabei mitnichten nur musikalische, sondern des Weiteren eben auch u.a. soziale, ökonomische, historische, technologische, mediale, visuelle und ideologische Faktoren zu einer relativ stabilen »Genrewelt «, wie Simon Frith sagt. ${ }^{12}$ In dieser Genrewelt wird in Pierre Bourdieus Sinne ökonomisches, kulturelles und soziales Kapital erworben und investiert. ${ }^{13}$ Sofern ihr eine Verstetigung gelingt, liegt der Schwerpunkt dieses Kapitalumschlags auf der Schaffung von Identifikationspunkten, auf der Strukturierung von Diskurs und, bar aller zur Verstetigung gleichfalls notwendigen Momente von Originalität und Dynamik, auf der Schaffung von Bedeutung durch die kontinuierliche Herstellung von Vertrautem. All dies erfolgt in musikalischen Genres natürlich nicht zuletzt auch durch das Normieren und Standardisieren musikalischer Traditionszusammenhänge, Verfahren und Materialien, die sich als genrekonform etablieren, wie beim Klavierquintett die Besetzungsfrage - ist aber eben nicht hierauf beschränkt oder gar damit identisch. Für die Verfasstheit des Genres des Klavierquintetts heutzutage ist das weitgehende Fehlen der Markennamen Klassischer Musik z.B. nicht weniger konstitutiv als die Besetzungsfrage.

Der Ausgangspunkt dafür, dass sich dieses Genre mit seinem Ensembleformat fest etablierte, wird gemeinhin an der Erstveröffentlichung von Robert Schumanns Klavierquintett Es-Dur im Jahr 1843 fest gemacht. ${ }^{14}$ Schumann hatte sein op. 44 einige Monate zuvor im September und Oktober 1842 als Geschenk für seine Frau, die Pianistin Clara Schumann, komponiert. Sie machte das Werk in den Folgejahren allgemein bekannt, indem sie damit durch ganz Europa tourte. ${ }^{15}$ Diese Arbeit initiierte nicht nur eine ganze Welle von Werken für Klavier plus Streichquartett. Der Trend blieb auch keine kurzzeitige Mode. Vielmehr stieß Schumann eine nachhaltig andauernde Produktivität an. ${ }^{16}$ Bis heute. So wird am Ende dieses Buches u.a. eine Reihe von Werken näher betrachtet, die nach dem Jahr 2000 entstanden sind. Mehr als anderthalb Jahrhunderte nach Schumann. Umgekehrt blickte das Klavierquintett zum Zeitpunkt von Schumanns genreprägender Komposition bereits auf ein gutes halbes Jahrhundert, wenn auch inkohärenter Vortradition zurück. ${ }^{17}$

Im Bereich des Klavierquintetts haben sich, von der Besetzungsfrage einmal abgesehen, im Gefolge Schumanns freilich keine allzu starken musikalischen Gattungsnormen ausgebildet. ${ }^{18}$ Das ist die erste Spezifik des Genres. Selbst die unmittelbare Vorbildfunktion der beiden meistgespiel- 
ten, -kommentierten und -aufgenommenen Kompositionen ihrer Art, Robert Schumanns op. 44 und Johannes Brahms' op. 34, ist begrenzt auf eine vergleichsweise kleine Gruppe eher unspektakulärer zentraleuropäischer Stücke der zweiten Hälfte des 19. Jahrhunderts. ${ }^{19}$ Die reiche Tradition im französischsprachigen Raum zum Beispiel entwickelte sich, wie die Forschung gezeigt hat, weitgehend unabhängig: Das gilt etwa hinsichtlich der in diesem Repertoire vorherrschenden, von raschen Akkordbrechungen geprägten Faktur des Klaviersatzes. ${ }^{20}$ Oder im Blick auf die Entwicklung »zur bestimmenden Gattung einer für den Konzertsaal geschriebenen Kammermusik « ${ }^{21}$ im letzten Drittel des 19. Jahrhunderts - eine Position, die in Deutschland das Streichquartett einnahm.

Die einzige Ausnahme als Gattungsnorm stellt eben der besagte Besetzungstypus dar, der sich mit Schumann durchsetzte. Klavier plus Streichquartett. Das Gros der Kompositionen, die man seit dem späten 18. Jahrhundert als Quintett für Klavier und Streicher publiziert und aufgeführt sieht, bedient sich noch des vollen Streichersatzes. Anstelle der zweiten Violine wie im Streichquartett ist ein Kontrabass vorgeschrieben. Das berühmteste Werk dieser Art ist Franz Schuberts sogenanntes »Forellenquintett« A-Dur D 667 von 1820, das einzige Werk diesen Besetzungszuschnitts, das sich noch im allgemeinen Konzert- und Tonaufnahmenrepertoire findet, dafür aber sehr häufig.

Der gattungsstiftende Erfolg von Schumanns op. 44 hat neben dem Umstand, dass es schlicht ein fulminantes Stück Musik ist, vor allem zwei Gründe: Erstens verbindet das Klavierquintett in seiner Besetzung zwei renommierte Standardformate des bürgerlichen Konzertlebens: den Solopianisten und das Streichquartett. Bis Mitte des 19. Jahrhunderts hatten sich feste Streichquartettensembles, die über Jahre in Ensemblenamen und Zusammensetzung der Instrumentalisten stabil blieben, bereits als ein solches Format etabliert. ${ }^{22}$ Klaviersolisten waren ohnehin seit langem fester Bestandteil des öffentlichen Konzertlebens.

Aber es war nicht nur eine strategisch kluge Formatidee, die Schumann nachhaltig popularisierte. Die vorausgegangenen Klavierquintette, gleich ob in der seinerzeit vorherrschenden Kontrabass- oder schon in der Streichquartettbesetzung, hatten bis Schumann durchweg konzertanten Charakter, nachdem sie sich um 1800 aus dem Kontext der Generalbassbegleitung emanzipiert und sich zugleich die zuvor oft unisono geführten tiefen Streicherpartien geteilt hatten. Es waren vorwiegend Miniaturklavierkonzerte, 
vielfach von führenden Klaviervirtuosen ihrer Zeit wie Jan Ladislav Dussek und Johan Baptist Cramer, Johann Nepomuk Hummel und Friedrich Kalkbrenner, John Field und Ferdinand Ries für den Eigengebrauch in räumlich kleineren Konzertrahmen verfasst oder als Arrangements von Klavierkonzerten oder Sinfonien angefertigt. Entsprechend fiel die Ensemblebehandlung aus, in welcher das Klavier mit virtuosen Passagen dominierte. Wechsel zwischen Klavier und Streichergruppe, wie sie typisch für virtuose Klavierkonzerte dieser Ära sind, prägen auch die Struktur dieser frühen Klavierquintette. Schumann brach mit diesem Modell radikal. Klavier und Streichquartett werden bei ihm nicht nur gleichberechtigt behandelt, sondern durch das ganze Werk hindurch miteinander verwoben, zu wenigstens der Tendenz nach gleichberechtigten Akteuren entwickelt. Dieser neue Ansatz erwies sich offenkundig als künstlerisch attraktiv, insofern er eine breite Produktion in Gang setze, die eben diese Idee aufgriff. Die Unabhängigkeit der fünf Instrumente und ihre gleichzeitige Balance in Kompositionsanlage und Klangbild ist ein zentrales Thema des Genres geblieben. ${ }^{23}$

Das Klavierquintett verbreitete sich als Gattung nach Schumann über ganz Europa. Umfängliche Repertoires entstanden im französischsprachigen und im deutschsprachigen Raum sowie vor allem ab der Jahrhundertwende in Russland, Großbritannien und später auch in den USA. Wir finden jedoch eine nennenswerte Produktion ebenso in Südeuropa von Spanien über Italien bis in den Balkan wie in Skandinavien oder in Tschechien und Polen vor. Das Klavierquintett ist auch in der sogenannten zeitgenössischen Musik respektive Neuen Musik nach dem Zweiten Weltkrieg ein regelmäßig genutztes und damit lebendiges Format geblieben. Selbst nach der Jahrtausendwende gibt es unermüdlich jedes Jahr Uraufführungen und Ersteinspielungen.

Das Klavierquintett ist also eine gut 200 Jahre alte Gattung der Klassischen Musik. Eine nachhaltige musikalische Praxis mit langer Tradition. Als Format immer noch aktuell und regelmäßig genutzt von Komponistinnen und Komponisten. Eine Erfolgsgeschichte, die seit langem kontinuierlich weiterläuft. Aber eine mit zwei Besonderheiten, konstitutiv für die Identität und Verfasstheit des Genres heute. Einer geradezu absurd engen Auswahl an Arbeiten, die die Aufmerksamkeitsschwelle der Leitmedien Klassischer Musik erreichen. Und einer Indie-Grass-Roots-Bewegung, die diese Zuspitzung seit gut einer Generation parallel zum Internetzeitalter systematisch unterläuft und das Genre über Tonaufnahmen erschließt. Für 
diese beiden Diagnosen interessiert sich mein besagtes Digital-HumanitiesProjekt.

Denn wie ich erst über die Jahre lernen sollte, ist jene Konstellation charakteristisch für die Gattung des Klavierquintetts und seine Rezeption insgesamt, auf die ich vor einem Vierteljahrhundert in der Musikabteilung der Lübecker Stadtbibliothek traf mit jener Aufnahme des Quintetto Fauré di Roma von Faurés Klavierquintett Nr. 2 c-Moll bei Claves. Musiker, von denen ich oftmals noch nie gehört habe, engagieren sich für Werke, die vielfach meine erste Berührung mit dem Schaffen einer Komponistin oder eines Komponisten darstellen. Und sie tun dies meist bei kleinen Labels, die ihre Nische jenseits der international gefeierten 〉Klassikstars` und säulenheiligen >Meisterwerke (gefunden haben. Oder werden gleich direkt im Internet veröffentlich, etwa auf Plattformen wie YouTube oder SoundCloud.

Wer sich über ein Format wie das Klavierquintett die Welt der Klassischen Musik erschließt, kann eine außerordentlich reiche mittelständische Musikpraxis entdecken. Wie bei spezialisierten Handwerksbetrieben oder Manufakturen wird hier exzellente Arbeit geleistet, in Komposition wie Interpretation wie Produktion. Doch sucht man nicht nach diesen Produkten, stößt man meist nur durch Zufall darauf. So wie ich damals in der Lübecker Stadtbibliothek. Oder wenn man gezielt nach ihnen fahndet, oft nur mit erheblichen Aufwand. So wie es nur Sammler tun. Sammler wie ich.

Tut man das, ist verschiedenes auffällig an dem dann sichtbar werdenden Genre: So z.B. der singuläre, oft bekenntnishafte Charakter der Werke, die Komponistinnen und Komponisten in diesem Format schaffen. Viele, für ihre jeweiligen Schöpfer zentrale frühe Werke finden sich z.B. in dieser Gruppe. Bei älteren Arbeiten ist dies oftmals sogar unmittelbar sichtbar anhand der Opuszahlen, so bei Alexis de Castillon (op. 1) und Jean Sibelius (o. op.), Joaquín Turina (op. 1) und Ernst von Dohnányi (op. 1), James Friskin (op. 1) und Louis Ferdinand von Preußen (op. 1), Miklós Rózsa (op. 2) und Christian Sinding (op. 5), Giovanni Sgambati (opp. 4 und 5) und Camille Saint-Saëns (op. 14), Josef Suk (op. 8), Ludwig Thuille (o. op.) und Ralph Vaughan Williams (o. op.). Diese Stücke stehen neben >letzten Worten reifer Künstler, so u.a. bei Gabriel Fauré und Edward Elgar, Sergej Tanejew, Morton Feldman und Olivier Messiaen, bei denen nach jahrzehntelangen Laufbahnen die jeweiligen Klavierquintette zu den allerletzten 
größeren vollendeten Arbeiten überhaupt gehören. Autobiographisch stark aufgeladene Stücke wie etwa bei Dmitri Schostakowitsch, Alfred Schnittke und Louis Vierne existieren ebenfalls viele. Genauso wie Kompositionen, an denen die jeweiligen Künstler oft Jahre, manchmal über Jahrzehnte hinweg arbeiteten wie bei Johannes Brahms und Nikolai Medtner, John Alden Carpenter und Wilhelm Furtwängler. Was es im Klavierquintett insbesondere ab Schumann hingegen wenig gibt, ist Ware von der Stange. Kaum lieblose Auftragswerke. Und nur selten Prestige suchende Stücke, die vor lauter Gattungstradition und Ringen um Aufnahme in den Kanon kaum laufen können. Eher scheint es so, dass wenn sich Komponistinnen und Komponisten dazu entschließen, ein Klavierquintett zu schreiben, es ihnen typischerweise ein Anliegen ist. Das Gros der Werke in diesem Genre, die ich kenne, wirkt zumindest so. Auf mich jedenfalls.

Ein weiterer Aspekt tritt an dieser Stelle verstärkend hinzu. Was ich zunächst nicht wusste, war, dass ich mit der Aufnahme von Faurés Musik durch das Quintetto Fauré di Roma eine Rarität in den Händen gehalten hatte: Es stellt eine Ausnahme dar, dass jemand wie Fauré gleich zwei oder gar noch mehr Klavierquintette hinterlässt. Es kommt vor, so wie bei Fauré. Vom 18. Jahrhundert bis ins 21. Jahrhundert hinein. Aber zwei scheint das Limit für Vielschreiber. So etwa bei Louise Farrenc (mit Bass), Franz Berwald und Antonín Dvořák, Giovanni Sgambati und Friedrich Gernsheim, Paul Juon und Ludwig Thuille, Charles-Marie Widor und Karl Goldmark, Ernst von Dohnányi und Max Reger, Cyril Scott und Hans Huber, Ernest Bloch und Grażyna Bacewicz, Mario Castelnuovo-Tedesco und Charles Wourinen, Migueal del Aguila und James Friskin, Vijay Iyer und Albin Fries. Schon ein zweites Stück in dieser Gattung zu schreiben, ist vergleichsweise selten. Darüber hinaus zu gehen aber handverlesen, beschränkt auf wenige Ausnahmen wie Salomon Jadassohn (3) und Bohuslav Martinů (3). Insgesamt ist dies eine Gattung, die von Solitären geprägt ist. Einzelstücken. Komponistinnen und Komponisten, die ganze Serien schreiben wie sonst an Opern oder Sinfonien, Streichquartetten oder Kunstliedern, Violinsonaten und Sinfonischen Dichtungen gibt es hier nicht mehr seit Luigi Boccherinis opp. 56 und 57 mit je 6 Kompositionen. So war es selbst bei Schumanns Klavierquintett Es-Dur op. 44 der Fall, das die Gattung revolutionierte. Denn trotz der großen Resonanz, den diese Arbeit unter Kollegen fand, sah sich weder Schumann noch ein anderer Komponist im näheren zeitlichen Umfeld veranlasst, eine Serienproduktion zu be- 
ginnen. Das war schon bei den Vorläufern zu Schumann so (Arrangements nicht mitgerechnet). Etwa den Quintetten für Klavier und Streicher von Komponisten wie Jan Ladislav Dussek und Friedrich Witt, Louis Ferdinand von Preussen und Ferdinand Ries, John Field und Johann Nepomuk Hummel, Antonín Reicha und Johann Baptist Cramer, George Onslow und Franz Limmer, Louis Spohr und Franz Schubert. Und so blieb es auch hiernach die Regel.

Wie all diese Namen von Komponistinnen und Komponisten bereits anzeigen und sich auf der Ebene von Interpreten und Labels, Spielstätten und Kommentatoren fortsetzt, ist das Klavierquintett aber vor allem weithin eine Welt jenseits der Markennamen Klassischer Musik, unter den Künstlern und Institutionen, den Kritikern wie den Wissenschaftlern. Selbst jenseits des musikgeschichtlichen Bewusstseins vieler, die sich mit Klassischer Musik professionell beschäftigen. All diese Werke und Tonaufnahmen erreichen nicht den Mainstream, die Leitmedien Klassischer Musik.

Unter dem Begriff Leitmedien fasse ich für hiesigen Zweck in einem weiten Sinne all jene Orte und Akteure zusammen, an bzw. von denen Musikgeschichte mit der Autorität, eine dafür maßgebliche Institution zu sein, geformt und tradiert wird. ${ }^{24}$ Dafür entlehne ich den Begriff der Publizistik und Medienwissenschaft, in welcher der Terminus für Medien gebraucht wird, die eine - Zitat des Medientheoretikers Udo Göttlich »Hauptfunktion in der Konstitution gesellschaftlicher Kommunikation und von Öffentlichkeit zukommt. $\ll^{25}$ Um eben jene Hauptfunktion geht es mir hier auch. Mich interessieren unter dem Begriff Leitmedien also jene Orte und Akteure, in bzw. von denen das gebildet und verhandelt wird, für das es in der Rechtswissenschaft einen vortrefflich präzisen Begriff gibt: die >herrschende Meinung $<$ - $\gg$ The artworld system ${ }^{26}$, wie es der Philosoph Noël Carroll in seiner Kritik an der Bestimmung des Kunstbegriffs durch Arthur C. Danto und George Dickie genannt hat. Der Mainstream des heutigen Klassikbetriebs.

Die Aufmerksamkeitsökonomie dieser Leitmedien mit ihren weitreichenden wirtschaftlichen, sozialen und kulturellen Konsequenzen ist in der Klassischen Musik in Werken wie Interpreten, Labels wie Spielstätten, Kommentatoren wie Publikationsrahmen ganz auf Markennamen zugeschnitten, die wiederum aus der Bedeutung der jeweiligen Instanz abgeleitet sind. ${ }^{27}$ Das jedenfalls führt das Genre des Klavierquintetts als Fallbeispiel vor. 
Überraschungserfolge von - aus Sicht des Mainstreams - No-NameProdukten gibt es auch in der Klassischen Musik. Ebenso wie One-HitWonder. Wie etwa die Einspielung von Henryk Góreckis Sinfonie Nr. 3 op. 36 (1977) im Jahr 1992 (Elektra Nonesuch 9 79282-2) von London Sinfonietta unter David Zinman mit der Sopranistin Dawn Upshaw, die sich siebenstellig verkaufte. ${ }^{28}$ Sie machte die Sängerin zum Star. Und das Werk eines polnischen Avantgardekomponisten zu einem Stück Popkultur. Aber dergleichen ist selten. Die Ausnahme, die die Regel bestätigt. Das Genre des Klavierquintetts hat in zwei Jahrhunderten keine einzige solche Ausnahme hervorgebracht. Die systemischen Mechanismen sind stark und selbst dann auf Markennamenprodukte konzentriert, wenn etwas Neues, Ungewöhnliches versucht wird, etwa, um mittels alternativer Marketingkonzepte oder Aufführungskontexte neue Hörerkreise für Klassische Musik zu erschließen. ${ }^{29}$ Auch das zeigt das Genre des Klavierquintetts in exemplarischer Weise.

Dergleichen im üblichen Blick der Leitmedien Klassischer Musik auf das Genre des Klavierquintetts wiederfinden und diagnostizieren zu können, ist natürlich nicht allzu überraschend. Vielmehr bestätigt sich hier vieles, was in einer kaum überschaubaren Fülle von kultursoziologischen Beobachtungen vom Kapitel Kulturindustrie Max Horkheimers und Theodor W. Adornos über Pierre Bourdieus Die feinen Unterschiede und Gerhard Schulzes Die Erlebnisgesellschaft bis zu Hartmut Böhmes Fetischismus und Kultur an vielen Stellen aufscheint. ${ }^{30}$ Das Interessante ist nicht der Befund an sich. Es ist die bedingungslose Konsequenz, mit der man diese Mechanismen im Fall des Klavierquintetts in den Leitmedien Klassischer Musik durchgeführt sieht. Das hat mit der besonderen Beschaffenheit dieses Genres zu tun. Den Beiträgen zu ihm über all die Jahrzehnte. Von wem diese stammen.

Dem Verständnis der Spezifik der Position des Klavierquintetts innerhalb jener Leitmedien kann man sich vielleicht am besten mittels eines Vergleichs annähern: Seiner Eigenart kommt recht nahe, wenn man an jemanden wie Birgit Fischer mit einer Sportart wie Kanu denkt. Deutschlands erfolgreichste Olympionikin überhaupt. Herausragend - aber allgemein unbekannt. Aufmerksamkeit Sport gegenüber ist ganz auf wenige Hauptsportarten konzentriert. Allen voran Fußball. Schon mit Abstand folgen Handball, Eishockey, Volleyball, Formel 1 oder Tennis. Bootsportarten sind demgegenüber eine Nische. Und innerhalb derer Kanu nochmals 
eine. Vom »Deutschlandachter« haben die meisten schon einmal gehört. Beim Kanu werden die Nichteingeweihten - also fast alle - erstmal überlegen, welche Paddelart damit gemeint ist. Und wie das Boot aussieht. Aber gesamtgesellschaftlich ist Kanu keineswegs zur Gänze irrelevant. Alle vier Jahre zu den Olympischen Spielen tritt es in den Vordergrund des Interesses. Bekommt Sendezeit und Berichterstattung. Sprich Aufmerksamkeit abseits der kleinen Spezialistenkreise. Ähnlich, wie es in den seltenen Fällen geschieht, in denen ein Klavierquintett von einem Meister Klassischer Musik mit berühmtem Namen verfasst wird, einem Robert Schumann etwa oder einem Johannes Brahms oder Antonín Dvořák. Bei einem Label wie der Deutschen Grammophon erscheint. Und dann Interpreten wie das Emerson String Quartet sein eigen nennt. Kanu wie Klavierquintett sind Nischenwelten mit einem deutlich sichtbaren, ritualisierten, aber letztlich punktuellen Zugang zu allgemeiner Relevanz, Resonanz und Anerkennung in ihren jeweiligen Bereichen: Sport hier, Klassische Musik dort. Über Großereignisse hier - und Markennamen dort.

Einige Beispiele mögen diesen Befund stellvertretend anhand von vier Bereichen näher belegen: Tonträgermarkt - Konzertwesen - Musikliteratur - Musikjournalismus. Ob in den Katalogen der Majorlabels oder bei YouTube. Ob in den Programmen der Carnegie Hall oder der kleinen Berliner Off-Klassik-Spielstätten von Schwartzscher Villa bis Heimathafen. Ob in kanonischen Musikgeschichtsbüchern oder in Kammermusikführern. Ob in den meinungsführenden Printfeuilletons oder auf speziell der Klassik gewidmeten Websites. Egal, wohin man schaut, gleichgültig, wie man die zu sammelnden Informationen dreht und wendet: Alle denkbaren Ansätze für derartige Stichproben führen zum selben Ergebnis. Das Genre des Klavierquintetts kommt in den Leitmedien Klassischer Musik nur in höchst selektiver Weise vor. Ungeachtet der Überfülle an verfügbaren Werken und mittlerweile auch an Tonaufnahmen, trotz über 200 Jahren Genregeschichte, trotz einer ungebrochen hohen kompositorischen Produktivität in diesem Format, trotz einer enormen Breite an vergleichbarer musikalischer Qualität: Besprochen, ediert, aufgenommen, aufgeführt, ja wahrgenommen sieht man in den Leitmedien Klassischer Musik nur Franz Schuberts »Forellenquintett« A-Dur D 667 (das schon seiner Besetzung mit Kontrabass wegen noch gar nicht recht zur Gattung gehört), Robert Schumanns op. 44 in EsDur, Johanes Brahms op. 34 in f-Moll, Antonín Dvořáks zweites Klavierquintett op. 81 in A-Dur und schließlich Dmitri Schostakowitschs Klavier- 
quintett op. 57 in g-Moll - >die üblichen Verdächtigen`, wie man sagen könnte. Mit schon einigem Abstand folgen heutzutage, quasi als zweite Reihe, die Klavierquintette von Edward Elgar in a-Moll op. 84, César Franck in f-Moll und Gabriel Fauré Nr. 2 in c-Moll op. 115 und vielleicht noch das zumindest häufig aufgenommene Klavierquintett von Alfred Schnittke stellvertretend für die Neue Musik nach 1945. Das war es. ${ }^{31}$

Tonträgermarkt - Eine klare Sprache spricht der Tonträgermarkt, d.h. die Quantität der Einspielungen und ihre Relation zueinander. Schon eine bloße Stichprobe über arkivmusic.com ermittelt für das Kernrepertoire stattliche Zahlen unterschiedlicher Einspielungen (zum Teil dort mit - hier herausgerechneten - Mehrfachveröffentlichungen vertreten):

- Schubert: 88

- Schumann: 64

- Brahms: 57

- Dvořák (Nr. 2): 44

- Schostakowitsch: 37

- Franck: 23

- Fauré (Nr. 2): 11

- Schnittke: 9

- Elgar: 8

Dass diese Zahlen dabei nur Stichproben sind, sieht man z.B. daran, dass arkivmusic.com für Schnittke 9 Tonträger dokumentiert, ich aber allein 20 kenne. ${ }^{32}$ Die tatsächlichen Zahlen sind also noch deutlich höher. Und das, bevor die übers Internet und die Rundfunkarchive zugänglichen Tonaufnahmen mitgezählt werden. Aber an dieser Stelle genügt, ein Gefühl für die Größenordnungen und ihre Relationen zu bekommen. Und da reichen bereits diese Zahlen. Denn die anderen Arbeiten, die im letzten Kapitel aufgelistet werden, verfügen nicht selten schon über keine Vergleichseinspielung. Ein Stück wie das Klavierquintett von Frank Bridge, von dem ich sechs Tonträger kenne, erscheint in diesem Kontext als ein vergleichsweise oft aufgenommenes. ${ }^{33}$ Das Gros der wissenschaftlich erfassten Klavierquintette wurde dagegen sogar noch gar nicht für den regulären Musikmarkt eingespielt. Man hat bei ihnen Glück, wenn man irgendwo eine Liveaufnahme im Internet oder einem Rundfunkarchiv dokumentiert findet. 
Stellvertretend illustrieren diese Aufmerksamkeitsökonomie die Kataloge der führenden drei Majorlabels, Universal, Sony und Warner, die gut Dreiviertel des Tonträgermarktes untereinander aufteilen. ${ }^{34}$ Eine strenge Hierarchie zwischen einem sehr eng begrenzten Kanon, den in der Regel die bekannten Interpreten der Häuser bedienen, wenn sie sich einmal dem Genre des Klavierquintetts zuwenden. Und dem Rest, der nicht systematisch gepflegt wird und oft nur zufällig, meist aber gar nicht die Programme dieser Firmen erreicht. Das Kernrepertoire des Genres ist bei allen drei Majors in dieser Weise jeweils klar als solches definiert mit dem Angebot von gleich mehreren, regelmäßig prominent besetzten Einspielungen von Schubert, Schumann, Brahms, Dvořák (Nr. 2) und Schostakowitsch.

Diese Bildung eines engen Kernrepertoires im Tonträgermarkt ist nicht neu für das Klavierquintett, wie man z.B. am Katalog des Deutschen Musikarchivs ablesen kann. In diesem werden seit einem halben Jahrhundert die von Unternehmen mit Sitz oder zumindest Niederlassung in Deutschland veröffentlichten Tonaufnahmen erfasst. Und dessen Bestand ist ebenfalls zunächst ganz auf die >üblichen Verdächtigen` hin konzentriert. ${ }^{35}$ Was umgekehrt anzeigt, dass dies das einzige war, was aus dem Genre heraus damals als Tonträger produziert wurde.

Daneben vertreiben die drei marktbeherrschenden Unternehmen zwar durchaus ausgewählte Aufnahmen weiterer Klavierquintette, so aktuell bei Universal Music Classical Aljabjew, Tanejew, Webern, Elgar, Bacewicz (Nr. 1 und 2) und Lewensohn, bei Sony Classical Franck, Fauré (Nr. 2), und Schmidt sowie bei Warner Classic Ries, Borodin, Franck, Zarębski, Fauré (Nr. 1 und 2), Dohnányi, Arensky, Tanejew, Bloch (Nr. 1), Granados, Korngold, Milhaud, Medtner, Tcherepnin und Adès. ${ }^{36}$ Die meisten dieser Aufnahmen finden freilich nur über größere CD-Boxen quasi der Vollständigkeit halber den Weg ins Programm. Oder sind gegenwärtig über die Majors lieferbar aufgrund der Übernahme älterer Kataloge Dritter. Oder sind als Einspielungsprojekte unschwer als persönliches Anliegen einem einzelnen prominenten Interpreten des jeweiligen Labels zuzuordnen und nicht als repertoireseitig liberale Programmpolitik zu interpretieren. So z.B. die Bacewicz-Aufnahmen ihres Landsmannes Krystian Zimerman bei der Deutsche Grammophon (Universal Music Classical). Das Album mit Einspielungen von Korngold (Klavierquartett für die linke Hand) und Franz Schmidt (Klavierquintett für die linke Hand) u.a. durch Leon Fleisher und Yo-Yo Ma bei Sony Classical. Oder die Darbietungen der Stücke von Ries, 
Borodin, Franck, Zarębski, Dohnányi, Arensky, Tanejew, Bloch, Granados, Korngold, Milhaud, Medtner und Schnittke, die allesamt dem jährlichen mehrteiligen Festivalbericht »Martha Argerich and Friends« vom Progetto Martha Argerich aus Lugano (2002-2016) zugehören (EMI bei Warner Classic). Das Beispiel Argerich zeigt obendrein, wie stark die Aktivitäten auch nur einer Künstlerin in einem in der Aufmerksamkeitsökonomie derart zugespitzt aufgestelltem Genre wie dem Klavierquintett das Gesamtbild beeinflussen, aber auch verzerren können.

Wo sich wenigstens die lieferbaren Tonträger im Allgemeinen und die Programme der Majors im Besonderen mit einiger Mühe noch einigermaßen gut aus verschiedenen Ressourcen zusammensuchen und auf die Frage nach der Teilhabe an diesem Teil der Leitmedien Klassischer Musik befragen lassen, wäre zusätzlich natürlich ein Abgleich mit anderen Hörangeboten sinnvoll. Also z.B. dem, was ausschließlich online zum Kauf angeboten wird, dem, was der Rundfunk an Repertoire überträgt und dem, was an Klavierquintetten über Streamingangebote genutzt wird, von Webradios bis YouTube und Spotify. Man vergleiche nur die zahlreichen, auf SoundCloud veröffentlichen zeitgenössischen Klavierquintette, von denen keine Aufnahmen den regulären Tonträgermarkt erreichen, um einen Eindruck davon zu bekommen, wie wichtig es wäre, auch solche Veröffentlichungsbereiche systematisch zu erfassen, wollte man insgesamt eine Bestandsaufnahme des Klavierquintettgenres erarbeiten. ${ }^{37}$ Hier sind aber auch noch viele weitere lohnende Folgefragen zu erkennen, etwa zum merklichen Kontrast zwischen den Verkaufszahlen von Tonaufnahmen klassischer Kammermusik, die sich typischerweise in den drei- bis maximal vierstelligen Größenordnungen von akademischen Publikationen bewegen, und den Streamingkennzahlen bei YouTube oder Spotify im regelmäßig fünf- und sechs- bis manchmal gar siebenstelligem Bereich gerade für die bekannteren Klavierquintette.

An dieser Stelle gibt es allerdings noch sehr viel zu tun für die Digital Humanities. Und an Music Data Mining im engeren Sinne ist da noch gar nicht gedacht. Etwa für Werkvergleiche oder Interpretationsforschung oder Höreranalysen. Denn schon auf der ganz basalen Ebene der Gewinnung von Primärinformationen über das gepflegte Repertoire sind die bislang ungelösten Herausforderungen enorm. Man denke nur an die eben angeführte Aufgabe, überhaupt einmal einen belastbaren Gesamteindruck von den medial verfügbaren Hörangeboten und ihrer Nutzung im Bereich des 
Klavierquintetts zu gewinnen. Etwa, um zu klären, inwiefern sich diese Aktivitäten zwar abseits der Majorlabels vollziehen, aber vielleicht aufgrund von Prestige und Resonanz auch zu den Leitmedien Klassischer Musik zu zählen wären. Bis dato lassen sich jedoch Annahmen darüber gar nicht so leicht überprüfen. Denn die Playlists der Rundfunksender der ARD-Anstalten sind z.B. nach gesendeten Stücken öffentlich nur für die jeweils vergangene Woche und dies mit erheblichem manuellem Aufwand zu durchsuchen. ${ }^{38}$ Oder man denke an YouTube, das via google allein für den Suchbegriff »Piano Quintet« 70.000 Treffer ausweist, die erst einmal technisch auf Einschlägigkeit hin zu sortieren und zu systematisieren wären. Von den erheblichen juristischen Herausforderungen für das Data Mining im Bereich der Tonaufnahmen ganz zu schweigen. ${ }^{39}$ Für hiesiges Anliegen stellt all das natürlich kein Problem und keinen Einwand dar. Ich weise dennoch hier explizit auch auf die Daten-, Forschungs- und letztlich Erkenntnislücken hin, weil es Perspektiven für digitale Musikwissenschaft auch jenseits ihres derzeitigen Kernarbeitsbereichs anzeigt, den hybriden und digitalen Ausgaben Klassischer Musik. ${ }^{40}$

Eine bereits jetzt mögliche Sortierung der Streamingkennzahlen nach quantitativer Resonanz, d.h. nach der Höhe der Aufrufe, zeigt z.B. exakt den skizzierten Kanon an. Und damit die ungebrochene, rezeptionsleitende Kraft der in den Leitmedien Klassischer Musik einzig unterstützten Markennamen auch unter den Bedingungen heutiger digitaler Musikdistribution im Internet. Bei YouTube z.B. sind die Top Ten, mit Aufrufzahlen zwischen 845.000 und 189.000, von Platz eins bis zehn runtergezählt Schubert (2x), Dvořák (Nr. 2), Brahms, Schuman, Elgar, Schubert, Dvořák (Nr. 2), Schostakowitsch und Brahms. ${ }^{41}$ Die nächsten Werke außerhalb dieser elitären Gruppe, die in der Liste erscheinen, sind dann wie zu erwarten, da insofern korrespondierend zum Tonträgermarkt, Fauré ( 168.000) und Franck ( 158.000) und, dann schon mit deutlicherem Abstand, Schnittke ( 62.000). Das Kernrepertoire wird erst viel später erstmals verlassen, mit Medtner ( 43.000) und Borodin ( 41.000), Ornstein ( 34.000, mit MarcAndré Hamelin am Klavier), Korngold ( 32.000) und Adès ( $\sim 31.000)$. Sowie einem kuriosen Gattungsbeitrag ( $\sim 33.000)$ aus dem später noch zu adressierenden Bereich der Neoklassik, das vom polnischen Pianisten Adam Sztaba stammt und in der polnischen Ausgabe der TV-Show Must Be The Music 2012 gezeigt wurde, in der Sztaba auch als Juror mitwirkte. ${ }^{42}$ Bis dahin und auch dazwischen erscheinen freilich noch zahlreiche weitere 
Interpretationen der >üblichen Verdächtigen`, die das Bild unübersehbar dominieren. Bei Spotify sieht es z.B. nicht anders aus.

Eine Stichprobe für das Jahr 2018 im Bereich Rundfunk, hier im öffentlich-rechtlichen Programm von BR Klassik, ergab ein korrespondierendes Bild. ${ }^{43}$ Die zu erwartenden Stücke dominieren. Ganz oder in Auszügen übertragen, entfiel auf vier der >Big Five mann (26), Dvořák (Nr. 2) (23), Schubert (16) und Brahms (7). Elgar (2) und Fauré (2 [Nr. 1], 1 [Nr. 2]) erhielten deutlich weniger. Ansonsten zu hören waren die Klavierquintette von Arensky (2), Dussek, Goldmark (Nr. 2), Hummel (2), Longo, Suk, Tanejew, Weinberg und Wolf-Ferrari. Wirkliche Überraschungen wie das Klavierquintett (1934) des Italieners Achille Longo (1900-1954) finden über Konzertübertragungen ihren Weg ins Programm, hier eines Konzerts mit der Pianistin Lauma Skride. Gemäß ihrer sonstigen Resonanz auffallend ist hingegen, dass Franck sowie die etwas >sperrigeren Modernen Schostakowitsch und Schnittke nicht gespielt wurden - eine statistische Auffälligkeit der Stichprobe, die in einer großen Datenerhebung zu überprüfen und ins Verhältnis zur allgemeinen Programmpolitik des jeweiligen Senders hinsichtlich Neuer und Neuester Musik zu stellen wäre. Die Dominanz von Schubert, Schumann, Brahms und Dvořák (Nr. 2) ist freilich überdeutlich.

Konzertwesen - Die Erstellung auswertbarer vernetzter Datenkorpora auch nur zu den wesentlichen, d.h. im Kontext der Leitmedien Klassischer Musik relevanten Aufführungsorte für Kammermusik in Deutschland steht noch aus. ${ }^{44}$ Die Informationslage ist noch viel schwieriger als im Bereich der Tonaufnahmen, das Potential für datengetriebene Geisteswissenschaft allein schon zur Herstellung eines differenzierten Gesamtbildes über die tatsächlich aufgeführte Musik innerhalb eines Genres wie dem Klavierquintett noch viel größer. Man schaue als Beispiel nur einmal, wie wenig statistisches Material das Deutsche Musikinformationszentrum derzeit an dieser Stelle zum Konzertwesen anbietet. ${ }^{45}$ Bislang fehlt es schon an einer zentralen Quellenerfassung für Ressourcen zu Konzertdaten, einem Pendant für Konzertprogramme zu RISM (schriftliche Primärquellen zur Musik), RILM (musikwissenschaftliche Sekundärliteratur) und RIdIM (musikalische Ikonographie). Hier gibt es tatsächlich für die Digital Humanities noch sehr viel Basisarbeit zu leisten. Insbesondere in der Vernetzung von Beständen. Aber auch auf der Ebene der Primärerschließung von Quellen hin zu computerunterstützt auswertbaren Informationen. Entsprechende Bemühungen 
laufen derzeit z.B. unter dem Stichwort musiconn.performance im Rahmen des Fachinformationsdienstes Musikwissenschaft und bei der GEMA. ${ }^{46}$ Der Bereich der datengetriebenen Musikereignisforschung steht jedoch noch recht am Anfang. ${ }^{47}$ Auch international ist er bislang auf Pilotprojekte beschränkt, die an Beispielen wie dem Wiener Musikverein oder der New Yorker Carnegie Hall aber bereits andeuten, was hiermit geleistet werden könnte. ${ }^{48}$ Etwa um zu verstehen, wie sich ein Genre wie das Klavierquintett im Konzertleben historisch entwickelt hat und heute platziert. Oder wie sich die Aufführungspolitik zu dem noch zu besprechenden aktuellen Trend im Tonträgermarkt verhält, das Genre des Klavierquintetts jenseits des Mainstream Klassischer Musik im Rahmen einer Indie-Grass-Roots-Bewegung zu erschließen. Oder um zu überprüfen, ob die behauptete markennamengetriebene Aufmerksamkeitsökonomie dem Klavierquintett gegenüber sich auch im Konzertwesen so wiederspiegelt. Das bereits bis 1891 zurück digital durchsuchbare Konzertarchiv der Carnegie Hall deutet z.B. genau hierauf hin. Die danach dort meistgespielten Stücke sind wenig überraschend: Schumann (47), Brahms (45), Dvořák (Nr. 2) (44), Schubert (37) und Schostakowitsch (16).

Bislang sind aber in der Regel nur aufwendig recherchierte Stichproben möglich, indem man z.B. die einzelnen Spielpläne einschlägiger Konzerthäuser und Festivals, Konzertagenturen und Kammermusikvereinigungen zu Rate zieht. Etwa sich stellvertretend durch die Programme der laufenden Spielzeit 2018/19 jener Institutionen arbeitet, die insoweit über das Deutsche Musikinformationszentrum erfasst sind. ${ }^{49}$ Oder sich durch ältere, z.B. partiell auch über den Datenbankdienst issuu.com zugängliche Saisonvorschauen liest. ${ }^{50}$

Für hiesigen Zweck genügen zur Illustration wieder einige repräsentative Schlaglichter auf typische Spielpläne in etablierten Häusern der Metropolen, um die grundsätzliche Situation des Genres zu belegen. Die nachfolgenden Konzerte sind alle Stand März 2019 über issuu.com in Saisonvorschauen nachgewiesen und wurden aufgrund der dortigen allgemeinen digitalen Zugänglichkeit für hiesigen Zweck exemplarisch ausgewählt. So waren z.B. zu hören in der

- Elbphilharmonie Hamburg:

- Spielzeit 2016/17: Arenksy und Schostakowitsch (11.4.2017) sowie Schubert (16.5.2017). 
- Spielzeit 2017/18: Schumann und Weinberg (19.10.2017), Dvořák (Nr. 2) (3.11.2017), Schostakowitsch (3.12.2017), Ginastera (25.1.2018 - Themenabend zum Komponisten), Dvořák (Nr. 2) (24.2.2018), Brahms (23.2.2018), Franck (3.4.2018) sowie Schumann (6.5.2018).

- Spielzeit 2018/19: Medtner (16.10.2018), Schubert (20.10.2018), Dvořák (Nr. 2) (27.11.2018), Fauré (Nr. 2) (9.12.2018) sowie Elgar (28.5.2019).

- Konzerthaus Berlin:

- Spielzeit 2014/15: Brahms (8.5.2015).

- Spielzeit 2018/19: Schubert (8.1.2019) sowie Brahms (11.5.2019).

- Spielzeit 2019/20: Brahms (19.11.2019) sowie Gubaidulina (29.3.2020).

- Philharmonie Berlin:

- Spielzeit 2018/19: Dohnányi (Nr. 2) (18.1.2019), Schumann (9.9.2018) sowie Trifonov (Artist-in-Residence) und Brahms (23.6.2019).

- Pierre-Boulez-Saal Berlin:

- Spielzeit 2018/19: Schumann (9.9.2018 - Boulez Ensemble XV in Philharmonie), Brahms (14.1.2019) sowie Schostakowitsch (23.2.2019).

- Wiener Konzerthaus:

- Spielzeit 2016/17: Weinberg und Brahms (4.12.2016), Schumann (26./27.2. 2017) sowie Dvořák (Nr. 2) (31.5.2017).

- Spielzeit 2017/18: Elgar und Schumann (27.4.2018) sowie Dvořák (Nr. 2) und Schostakowitsch (14./15.5.2018).

- Spielzeit 2018/19: Schumann (21.10.2018), Schumann (14.12.2018), Schostakowitsch (24./25.2.2019) sowie Schostakowitsch (7.4.2019).

- Tonhalle Zürich:

- Spielzeit 2014/15: Schnittke (5.2.2015).

- Spielzeit 2015/16: Fauré (Nr. 2) (26.11.2015), Schubert (17.1.2016), Dohnányi (Nr. 1) (29.5.2016) sowie Ives und Martin (2.6.2016).

- Spielzeit 2016/17: Schumann (17.4.2017) sowie Bloch (Nr. 2) (25.6.2017).

- Spielzeit 2017/18: Schostakowitsch (21.1.2018) sowie Dvořák (Nr. 2) (2.4. 2018).

- Spielzeit 2018/19: Enescu (14.10.2018) sowie Nowakowski (29.11.2018).

Korrespondierend weist z.B. die in der Hauptstadt sehr präsente KonzertDirektion Hans Adler per März 2019 auf ihrer Website für Berlin für die laufende und vorherige Spielzeiten Aufführungen der Klavierquintette von Schubert, Schumann, Brahms, Franck, Dvořák (Nr. 2) (3x), Dohnányi (Nr. 2), Tanejew und Schostakowitsch aus. ${ }^{51}$ Einen Eindruck für eine Langzeit- 
perspektive vermittelt hierneben z.B. ein Blick in das Repertoire, das bei der seit 1988 in Berlin bestehenden Kammermusikreihe Spectrum Concerts (mit Teilschwerpunkt US-amerikanische Musik) gegeben wurde: Arensky (3x), Bartók, Brahms (5x), Dvořák (Nr. 2) (3x), Franck, Korngold (2x), R. Liebermann, Schnittke, Schubert (4x), Schumann (2x), Schostakowitsch (7x), Tanejew (2x) und Toch (2x). ${ }^{52}$ Ein Blick stellvertretend in die vergangenen sechs Monate (11/2018-04/2019) der Regionalausgabe Berlin/Brandenburg des Veranstaltungsmagazins Concerti zeigt analoges, wonach in diesem Zeitraum, insbesondere auch in den hier zusätzlich erfassten kleineren Spielstätten, folgende Stücke zu hören waren: Brahms (4.12.2018), Bridge und Schumann (15./16.12.2018), Schubert (8.1.2019), Brahms (12.1.2019), Dohnányi (Nr. 2) und Dvořák (Nr. 2) (18.1.2019), Brahms (27.1.2019), Schostakowitsch (23.2.2019), Brahms (16.3.2019) sowie Dvóřak (Nr. 2) (3.4.2019)..$^{53}$

Schon in diesen wenigen Schlaglichtern zum Konzertwesen wird die Stabilität des Kernrepertoires deutlich sichtbar. Ebenso, dass die renommierten Interpreten fast ganz auf dieses konzentriert sind - und nicht, was anzunehmen vielleicht nahe läge, ihr Prestige nutzen, seltener zu hörende Stücke bekannter zu machen. Auch deutet sich hier bereits an, dass der derzeitige, noch näher zu beschreibende Boom im Genre des Klavierquintetts an Aufnahmen auch sehr weit abseits des Kanons die Konzertprogramme noch nicht in nennenswerter Weise erreicht. Gemessen am Status quo sind die Kammermusikkonzerte des Deutschen Symphonie Orchesters Berlin, die z.B. seit der Spielzeit 2014/15 Ankündigungen für Aufführungen der Klavierquintette von Fauré (Nr. 2) (10.4.2015), Kapustin (16.9. 2016), Vaughan Williams (7.5.2017), Brahms (15.6.2018) sowie Pfitzner (28.4.2019) enthielten, schon ein rares Beispiel für eine vergleichsweise progressive Programmpolitik. Und nur der fulminante russische Jazzpianist und -komponist Nikolai Kapustin dürfte hier für die meisten Klassikinteressierten wirklich ein unbekannter Künstler sein. Vertreten mit einem sehr originellen Genrebeitrag, auf den ich noch zu sprechen kommen werde.

Musikliteratur - Mit dem Musikschrifttum verhält sich nicht anders als mit dem Tonträgermarkt und dem Konzertwesen. Eher wird die Auswahl noch strenger gehandhabt. Das wird sichtbar, egal in welche Standardmonographien, -handbücher und -enzyklopädien zur allgemeinen Musikgeschichte man schaut. Auch hier mögen im Kontext des hiesigen Anliegens wieder stellvertretend einige Schlaglichter genügen. 
Je allgemeiner und zugleich komprimierter eine Musikgeschichte auftritt, desto unwahrscheinlicher ist es, dass das Klavierquintett überhaupt gestreift wird, von Hans Heinrich Eggebrechts Musik im Abendland über Werner Keils Musikgeschichte im Überblick bis zu Alex Ross' The Rest is Noise. ${ }^{54}$ Das von Carl Dahlhaus herausgegebene dreizehnbändige Neue Handbuch der Musikwissenschaft z.B. führt trotz eines 408-seitigen Registerbandes nicht einmal einen Registereintrag zum Klavierquintett. ${ }^{55}$

Wenn das Genre berührt wird, dann regelmäßig mit den >üblichen Verdächtigen . Von Richard Taruskins Oxford History of Western Music (Brahms, Dvořák und Franck) ${ }^{56}$ und J. Peter Burkholder, Donald Jay Grout und Claude V. Paliscas Nortons History of Western Music (Schubert, Brahms, Beach $)^{57}$ über Paul Griffiths $A$ Concise History of Western Music (Schumann und Franck) ${ }^{58}$ und Walter Frischs Music in the Nineteenth Century. Western Music in Context (Schubert, Brahms) ${ }^{59}$ bis $\mathrm{zu}$ den beiden Bänden der Cambridge History zur Musik des 19. und 20. Jahrhunderts (Schumann, Brahms). ${ }^{60}$ Überall dasselbe Bild. In populärwissenschaftlichen Werken verhält es sich im Übrigen erst recht nicht anders. Man vergleiche nur Matthew Ryes 1001 Klassik-Alben, die sie hören sollten, bevor das Leben vorbei ist, Arnold Werner-Jensens Das Reclam Buch der Musik oder Kurt Pahlens Die große Geschichte der Musik. ${ }^{61}$

An Monographien zum Genre oder zumindest zu Teilrepertoires sind, soweit ich sehe, in den vergangenen 50 Jahren überhaupt nur drei veröffentlich worden. ${ }^{62}$ Und auch hier sind Schumann und Brahms die Fixpunkte. Um ein Gefühl für die völlig anderen publizistischen Größenordnungen im Mainstream Klassischer Musik zu bekommen, erinnere man sich nur an die Hundertschaft neuer Monographien und Sammelbände zu Richard Wagner aus Anlass des Jubiläumsjahres 2013. ${ }^{63}$ Oder an den Umstand, dass auch ohne einschlägiges Komponisten- oder Werkjubiläum seit dem Mauerfall allein zu Beethovens Neunter Sinfonie im Schnitt alle zwei Jahre eine Monographie veröffentlicht wurde. ${ }^{64}$

Die Artikel zur Klavierkammermusik in der über 20.000 Artikel und 25.000 Seiten Umfang verfügenden zweiten, aktuellen Ausgabe der Enzyklopädie Die Musik in Geschichte und Gegenwart und Ludwig Finschers 121 Seiten starke Einführung »Gattungen und Besetzungen der Kammermusik» im über 1200 Seiten umfassenden Reclams Kammermusikführer widmen der Angelegenheit ein wenig mehr Aufmerksamkeit und nennen zumindest deutlich mehr Komponistennamen. ${ }^{65}$ Freilich stellen sie insgesamt keine 
Ausnahmen von der Regel dar. Die jeweiligen Einführungen in die Gattung gehen nicht tiefer als >Name-dropping〈. Wie sollen sie auch? Bar des Umfangs dieser beiden Gesamtwerke umfassen die Abschnitte zur Gattung des Klavierquintetts und seiner über 200jährigen Geschichte und Vorgeschichte in beiden Fällen jeweils knapp fünf Seiten zuzüglich der einen oder anderen Bemerkung in Komponistenartikeln bzw. Einzelwerkbesprechungen. Der Eintrag "piano quintet" von David Fenton im New Grove Dictionary of Music and Musicians ist noch kürzer und ganz auf Namenslisten konzentriert. $^{66}$

Ergänzt wird das Musikschrifttum um eine Reihe von Fachaufsätzen zu Einzelwerken, typischerweise wiederum mit Fokus auf die bekannten Komponisten und Werke. ${ }^{67}$ Ausnahmen wie Thomas Adès betreffen vor allem zeitgenössische Musik. Man vergleiche zu solchen wissenschaftlichen Artikeln und ihren Schwerpunkten stellvertretend die bibliographischen Angaben hierzu, die jeweils den Werkportraits am Ende des Buches beigegeben sind.

Und immer wieder findet man kurze Analysen zu Stücken oder Interpretationen in Büchern über einzelne Komponisten, Performer oder in den großangelegten, systematisch konzipierten Musikgeschichtsprojekten wie dem vierzehnbändigen Handbuch der Musik im 20. Jahrhundert oder dem siebzehnbändigen Handbuch der musikalischen Gattungen. ${ }^{68}$ Oder in National- und Regionalmusikgeschichten wie der Geschichte der russischen und sowjetischen Musik von Dorothea Redepenning. ${ }^{69}$ Informationen jenseits des Kernrepertoires sind jedoch meist mühsam zu ermitteln und mitunter sogar nur in archivbasierten, unveröffentlichten Dissertationen zu finden. Oder durch eigene Primärquellenforschung.

Selbst die dezidiert der Kammermusik gewidmeten Führer konzentrieren sich auf das gängige Repertoire, bestätigen und tradieren es dadurch zugleich, so z.B.

- Ingeborg Allihns Kammermusikführer: >Big Fiveく plus Borodin, Elgar, Farrenc, Meyer und Zarębski;

- Harenberg Kulturführer Kammermusik: >Big Five` plus Elgar, Fauré (Nr. 1 und 2), Franck, Reger (Nr. 1 und 2) und Spohr;

- Melvin Bergers Guide to Chamber Music: ‘Big Five〈;

- James M. Kellers Chamber Music: A Listener's Guide: >Big Fiveく plus Elgar, Fauré (Nr. 2) und Franck; 
- Lucy Miller Murrays Chamber Music: An Extensive Guide for Listeners: >Big Five` plus Adès, Bloch (Nr. 1), Dohnányi (Nr. 1 und 2), Elgar, Fauré (Nr. 1 und 2), Foote, C. Franck, E. Franck, Korngold, Schnittke und Vaughan Williams. ${ }^{70}$

Ein Musterbeispiel für all dies ist der gut informierte Artikel von Colin Lawson im Cambridge Companion to String Quartet. ${ }^{71}$ Die skizzierte Gewichtung zwischen ausführlicher betrachtetem Kernrepertoire und auf Namensnennung und Nebensätze reduzierter Genreperipherie ist hier geradezu idealtypisch umgesetzt.

Musikjournalismus - Die zentralen Feuilletons wie hierzulande DIE $Z E I T$, die $F A Z$ und die $S Z$ zeigen keinen anderen Befund. Ebenso wenig wie die auf Klassische Musik spezialisierten Magazine und Onlineangebote. Ein klarer Fokus auf das skizzierte Kernrepertoire mit einer Ausnahme hier und da ist auch hier vorherrschend. Bunter wird es meist erst, wenn das Medium wie nmbx.newmusicusa.org einen klaren Schwerpunkt auf zeitgenössischer Musik hat. Stellvertretend sei auf DIE ZEIT verwiesen, die in den vergangenen 20 Jahren in Konzert- und vor allem Plattenkritiken wiederholt das Genre des Klavierquintetts berührt hat, aber wie zu erwarten steht mit klarem Fokus auf das besagte Kernrepertoire: Schubert, Schumann (mehrfach), Brahms (mehrfach), Schostakowitsch sowie Elgar, Fauré (Nr. 1 und Nr. 2) und Schnittke, dazu als Raritäten Joachim Raff, August Klughardt, Anton Webern, Wilhelm Furtwängler, George Enescu und Thomas Adès. ${ }^{72}$ Eine erste Stichprobe im digitalen Tageszeitungsarchiv der Deutschen Nationalbibliothek in FAZ, Tagesspiegel und SZ ergab ein korrespondierendes Bild. Das seltene Auftauchen von Klavierquintetten abseits des Kernrepertoires beschränkt sich fast ausschließlich auf die Ankündigung von Aufführungen oder Übertragungen in TV und Rundfunk, ohne nähere musikjournalistische Besprechung oder Einordnung.

All dies sind natürlich Schlaglichter. Stichproben. Eine systematische empirische Untersuchung mit den Mitteln der Digital Humanities steht noch aus. In den zu diesen vier und weiteren Teilbereichen der Leitmedien und darüber hinaus bereits vorhandenen oder noch zu erstellenden thematisch einschlägigen Datenkorpora. Sie ließe sich geraume Zeit fortsetzen und datenseitig nach Leitmedien, Indieszene, Untersuchungszeitraum, Untersuchungsorten und -regionen usw. umfänglich ausweiten, ausdifferenzieren und vertiefen. Und um analoge Studien zu Vergleichsgenres wie dem Streichquartett ergänzen. $\mathrm{Zu}$ eruieren, wie weit man damit kommt, ist 
u.a. Aufgabe meines eingangs erwähnten laufenden Pilotprojekts an der Deutschen Nationalbibliothek. Um die empirische Basis für die vorherigen Aussagen zu stärken. Aber umgekehrt auch, um die Eigenart und Aussagekraft der hauseigenen Sammelschwerpunkte und digitalen Datenbanken zu verstehen.

Die Rezeptionssituation des Klavierquintetts stellt mit ihrem eben skizzierten Status quo keine Ausnahme dar. Vielmehr treten hieran Mechanismen zu Tage, der allgemein bestimmend sind für den heutigen, markennamengetriebenen Mainstream Klassischer Musik. Der im Übrigen schon seit langem so funktioniert. In der spezifischen Rezeption dieses Genres scheint also etwas Generelles auf. Deutlicher aber als vielleicht andernorts aufgrund einer Eigenart der Geschichte dieses Genres. Der Anomalie, dass in einer jahrhundertelangen Produktion ausgerechnet die berühmten Leitkomponisten so auffallend und konsequent fehlen. Selbst jene, die viel und profiliert Kammermusik verfasst haben.

Diese Anomalie selbst ist sicher zufällig. Dafür, dass man es mit etwas anderem zu tun hat, gibt es keinerlei belastbare geschweige denn verallgemeinerbare Hinweise. Das Klavierquintett genoss nie die schlechte Reputation einer Gattung, die höchsten Kunstansprüchen nicht genügen könne. Im Gegenteil liegt es nahe, für diese Gattung zu schreiben, da sowohl Solopianist als auch Streichquartett lange etablierte Standardformate des bürgerlichen Konzertlebens sind. Prädestiniert für Arbeiten von höchstem ästhetischem Prestige. Und reich hieran. Von vielen der stärksten Markennamen unter den Leitkomponisten auch bedient.

Aber mag diese Anomalie Zufall sein. Dass also gefühlt die meisten, an Kammermusik interessierten Komponistinnen und Komponisten zweiter, dritter, vierter und oft gar keiner Prominenz sich beteiligt haben. Und ausgerechnet die Markennamen nicht. Die Konsequenz, die daraus gezogen wird und in dem skizzierten Rezeptionsbild aufscheint, in den Leitmeiden Klassischer Musik ausschließlich Markennamen zu beachten, kann es aber nicht sein. Alle wenigen Werke von Komponisten ersten Ranges und Renommees sind in den Leitmedien präsent? Ohne Abstriche? Ausschließlich die? Nicht ein einziges >One-Hit-Wonder das es in diesen Kreis geschafft hat? Kein einziges Werk aus 200 Jahren eines ansonsten vielleicht unbeachteten Komponisten, das lohnt? Das auf Augenhöhe steht. Kein Alexis de Castillion, Wilhelm Berger, Frank Martin, Giuseppe Martucci, Nikolai Medtner, keine Addela Maddison, Amy Beach, 
Grażyna Bacewicz, Sofia Gubaidulina, kein Juliusz Zarębski, Sergej Tanejew oder Arnold Bax - um nur einige der von ihren Genrebeiträgen her durchaus naheliegenden Kandidatinnen und Kandidaten zu nennen?

Das nun ist eben keinem Zufall mehr geschuldet. Das hat System. Markennamen werden in der Klassik von der Vorstellung von Bedeutung getrieben und davon abgeleitet mit dem Versprechen auf besonders hochwertige Qualität verbunden. Sie seien besser. Historisch und ästhetisch, sozial und pädagogisch relevanter. Mit ihnen gilt es sich zu beschäftigen. Bedeutung kann dabei verschieden begründet sein, wie sich stellvertretend an Werken zeigen lässt. Etwa darüber, »die Stellung zu bestimmen, die [ein Werk] im geschichtlichen Prozess einnimmt « ${ }^{73}$ (Carl Dahlhaus) oder über eine »Vorstellung von musikalischen Kunstwerken, deren Wert gerade nicht verhandelbar, sondern intrinsisch ist» (Friederich Geiger/Tobias Janz). ${ }^{74}$ Auf die genaue Begründung der Bedeutung kommt es letztlich nicht an. So oder so wird Bedeutung der jeweiligen Instanz eingeschrieben und sie damit zum Markennamen verfestigt, die - das ist der an dieser Stelle entscheidende Aspekt daran - für eine besonders hochwertige Qualität steht. In den Markennamen finden wir also die »Stabilität, Identitätsstiftung und Wertbeständigkeit als Funktionsmerkmale des Kanons« wieder, der als ein Faktor neben anderen im Markennamensystem aufgeht. ${ }^{75}$ Das System ist in seinen Bestandteilen, sprich den jeweils prägenden Markennamen, dynamisch, so wie es das Kanondenken im engeren Sinne auch stets war. ${ }^{76}$ Und es sind eine Vielzahl an Akteuren und Einflussfaktoren zu bedenken, wie etwa Richard A. Peterson und Narasimhan Anand gezeigt haben. ${ }^{77}$ Aber das Spiel bleibt das Gleiche. Und es ist die Qualitätsunterstellung Markennamen gegenüber, die im Kontext Klassischer Musik in ihren Leitmedien entscheidend ist, das »unhintergehbare Argument ästhetischer Qualität« (Melanie Wald-Fuhrmann). ${ }^{78}$

Den Status quo des Klavierquintetts in den Leitmedien Klassischer $\mathrm{Mu}-$ sik zu hinterfragen, die Musikgeschichte des Klavierquintetts, die dort konturiert und tradiert wird, ist daher natürlich überhaupt nur interessant, wenn sich der Eindruck aufdrängen sollte, dass dort Ware zweiter Qualität bevorzugt behandelt wird, ausschließlich, weil Markennamen sie verantwortet haben. Oder weil die Produktion des Genres im Übrigen eben nicht durchweg spürbar schlechter wäre als das Kernrepertoire. Denn natürlich ist es grundsätzlich denkbar, dass die Markenprodukte, von Dvořák oder Schostakowitsch, Schumann oder Brahms, schlicht tatsächlich jene mit der 
höheren Qualität sind: Bessere Stücke. Bessere Interpretationen. Bessere Aufnahmen. Bessere Kommentierungen. Größere Wirkung.

Will man das Klavierquintett als Genre verstehen, seine Gegenwart beschreiben, seine Geschichte erzählen, ist diese Frage nach der Zuspitzung ausschließlich auf Markennamen in den Leitmedien zu stellen. Die heikle, selten einmal explizit gemachte, meist nur in der immer gleichen Auswahl implizit werdende Qualitätsunterstellung zu adressieren. Man kann ihr nicht ausweichen. So dominant ist in seiner öffentlichen Wahrnehmung die Bildung jenes Kernrepertoires >großer Namen für dieses Genre. Eine Auswahl, die jedenfalls zunächst einmal eben nur über die beteiligten Markennamen ausgezeichnet und als solche nachvollziehbar gemacht ist.

Ästhetische Qualität? Ästhetisches Erleben vielleicht sogar? An dieser Stelle kommt die Planung meines Digital-Humanities-Pilotprojekts natürlich an eine Grenze. Und setzt das Nachdenken über die selbige ein. Denn wenn der Schlüssel zum Verständnis der Verfasstheit der Gegenwart des Klavierquintetts als Genre besagte Qualitätsunterstellung ist, muss man sich dann nicht der Frage ästhetischer Qualität stellen? Sich fragen, was das heißen kann, auf Produktions- wie Rezeptionsseite? Das ist keine bloß rhetorische Frage. Auf letzteres werde ich tatsächlich später versuchen, eine konkrete Antwort anzubieten.

Man muss dies nicht tun. Die Anwendung digitaler Analyse- und Interpretationsmethoden auf große Datenmengen wie z.B. große Audio-, Video-, Noten- und Textarchive (hier z.B. Tageszeitungen, Konzertprogramme, Briefe, wissenschaftliche Fachliteratur, Foren- und Blogdiskussionen) als ein Kernbereich dessen, was unter dem Lemma Digital Humanities getan werden kann und getan wird, von Text-, Bild- und Audio- über Sentimentund Netzwerkanalyse bis Mapping, Stilometrie und Topic Modeling, bieten auch so einiges an, wenn man sich im Fall des Klavierquintetts dem skizzierten Rezeptionsbild mit seiner inhärenten Qualitätsunterstellung annähern will. ${ }^{79}$ Das gilt insbesondere, wenn man diesen Status quo als historischen und soziokulturellen Fakt hinnehmen, einen Schnitt machen und sich auf die Aufgabe konzentrieren, aber zugleich eben auch beschränken will, den angedeuteten Tanz um ein maximal zugespitztes Kernrepertoire möglichst umfassend zu beschreiben, zu belegen und zu dokumentieren. Es ist natürlich immer eine sinnvolle Aufgabe, Bauchgefühl und Stichproben sofern möglich auf eine verlässliche empirische Basis zu stellen. Aussagen werden belastbarer. Und oftmals Folgefragen mit ganz anderer Präzision 
stellbar, bisweilen überhaupt erst als solche sichtbar. Auch für die Musikwissenschaft und das bis ins musikalische Material hinein, wie z.B. das Projekt Dig That Lick für die Jazzforschung vorführt. ${ }^{80}$ Ebenso unmittelbar einschlägig erscheint der Mehrwert der Digital Humanities an dieser Stelle für Diskursanalysen, die über ausgewählte repräsentative Leitmedien wie die meinungsführenden Feuilletons und kanonischen Musikgeschichten hinausgehen wollen. Etwa zu der Frage, ob sich die Akteure des Genres über die Jahrzehnte hinweg im Zusammenhang mit ihren Aktivitäten in der Gattung korrespondierend zum skizzierten Status quo geäußert haben? $\mathrm{Ob}$ sie darauf reagiert haben? Ob sie ihn überhaupt als solchen, vielleicht sogar als Problem wahrgenommen haben? Oder es heute tun? Oder man könnte an die Frage denken, wie die diesen Status quo tragenden Leitmedien argumentativ und personell miteinander vernetzt sind? Oder was die Fachcommunity jenseits der wenigen kanonischen Leitautoren in Musikwissenschaft und Musikjournalismus sagt. ${ }^{81}$ Oder welche ästhetischen Kriterien auf Nutzerseite vorgebracht werden, etwa in Foren der Massenkommunikation wie den Kommentarlisten von YouTube. ${ }^{82}$ Hier lässt sich mit Gewinn in viele Richtungen denken. ${ }^{83}$ Was u.a. im besagten Pilotprojekt auch passiert.

Nur: Der für die Gegenwart des Genres essentiellen Regulierungswirkung der Markennamen und der mit ihnen verbundenen Qualitätsunterstellung dürfte man auf diese Weise am Ende nicht substantiell näher gekommen. Da muss man sich nichts vormachen. Es gibt keinerlei Anlass, anderes zu erwarten. Von mehr Daten, von einer besseren Datenerschließbarkeit. Zu einheitlich ist das Bild. $\mathrm{Zu}$ verlässlich erscheinen die Stichproben. $\mathrm{Zu}$ implizit - stillschweigend - regelmäßig das Handeln. Zu konstant aber in der Aussage. Egal, in welche Richtung man sich wendet: Je tiefer man bohrt, desto mehr verfestigt sich das Bild.

Das es das tut, ist auch nicht das Problem. Es ist halt, was es ist. Abgesehen davon, dass anderen vorzuhalten, wie unvertretbar ihr Kanon ist und nur den eigenen wertvoll und begründet - und sich selbst erleuchtet - zu finden, zwar ein traurig beliebter Zeitvertreib ist. Aber halt nicht nur zu nichts führt. Sondern vor allem in seiner Inkonsequenz unaufrichtig, anmaßend und unproduktiv ist, die Bruno Latour so beherzt beschrieben hat. ${ }^{84}$ Entsprechend motiviert mich keine Revisionsagenda, wenn ich bei besagter, durch Markennamen abgesicherter Qualitätsunterstellung stutze. Und weiterfragen möchte. Ich habe also kein Problem damit, wenn Leute nur 
Schumann und Brahms, Schubert, Dvořák und Schostakowitsch aufführen, aufnehmen und kommentieren wollen. Sollen sie. Dies ist kein Plädoyer für eine vermeintlich vernachlässigte Gattung und angeblich verkannte Kleinmeister. Mein Anliegen ist es nicht, einen Kanon durch einen anders zusammengesetzten auszutauschen. Mich interessiert an dieser Stelle im Gegenteil, dass die Dinge in der beschriebenen Weise sind wie sie sind. Nicht, um sie zu dekonstruieren. Und über digitale Methoden vielleicht Munition dafür zu gewinnen. Etwa, um das Markennamenspiel als Elitenprojekt jenseits der Hörer dieser Musik zu entlarven. Über den Vergleich der Leitmedienpolitik mit dann ihr möglicherweise widersprechenden Kommentaren im Internet oder Verkaufszahlen (was alle Stichproben nicht erwarten lassen). Nein, was mich interessiert, ist der Konflikt mit meinem so anderen eigenen ästhetischen Erleben dieses Genres. Das so gar nicht zu der skizzierten Situation passen will. Und von besagter Qualitätsunterstellung provoziert wird. Höre ich so falsch?

Wie soll diese Dimension des Ganzen aber ins Verhältnis gesetzt werden zu dem, was ich im besagten Pilotprojekt mit den Methoden der Digital Humanities anstrebe? Oder ist im Gegenteil auf diese Dimension zu verzichten, sie als irrelevant oder gar als wissenschaftlich inadäquat zurückzuweisen? 


\section{Exkurs I: What of That?}

\section{Zur Frage nach den Möglichkeiten und Grenzen der Digital Humanities für die Musikgeschichtsschreibung}

Das ist das Problem: Die Qualitätsunterstellung. Die Frage, ob die übrige Produktion des Genres tatsächlich durchweg derart schlechter ist, wie die Pflege primär des rigiden Kernrepertoires impliziert. Das Unbehagen, das daraus resultiert, dass das eigene ästhetische Erleben dieses Genres so gar nicht zu der skizzierten Rezeptionslage und Markennamenfixierung passen will. Und die Herausforderung, der ich mich deswegen gegenübersehe. $\mathrm{Zu}$ klären, ob das eigene ästhetische Erleben an dieser Stelle eine Rolle spielen darf. Vielleicht aber auch gerade muss. Für mich als Musikhistoriker. In einem musikhistorischen Projekt, das sich für die Entwicklung und Gegenwart eines Genres interessiert. Und dafür im Kontext der Digital Humanities operiert. Nach Erkenntnis in großen Datenmengen sucht. Hier scheint eine Grenze erreicht. Eine Konfliktlinie berührt, die Barbara Flückiger in aller Schärfe umrissen hat:

»Zwischen dem Anspruch ästhetische Phänomene detailliert zu analysieren und dem Einsatz von computer-gestützten Verfahren scheint es eine grundlegende Diskrepanz zu geben. Denn subtile Details der Gestaltung erfordern die Kontextualisierung und Differenzierung durch ein menschliches Subjekt, während digitale Systeme streng formalisierte Verfahren der Erfassung und Prozessierung von Daten verlangen. $\ll^{85}$ 
Sich über dieses Genre selbst hörend ein Urteil bilden zu können, um z.B. zu einer Einschätzung über diese musikalische Praxis zu gelangen wie ich sie eben vertreten habe - dass mein ästhetisches Erleben nicht zum kohärenten Leitbild des Mainstreams passt -, ist überhaupt erst seit kurzem möglich. Und damit zu der Herausforderung zu gelangen, vor der ich hier stehe. Entgegen der beschriebenen Verfasstheit der Leitmedien Klassischer Musik hat sich in den vergangenen gut 20 Jahren nämlich eine Art IndieGrass-Roots-Bewegung entwickelt. Die das in den Leitmedien ausgegebene Gebot eines streng im Fokus zu haltenden Kernrepertoires unterläuft. Und auch die Randbereiche des Genres inzwischen in einer Weise in Noten und insbesondere zum Hören erschlossen hat, dass die Grenze dessen erreicht ist, was ein Einzelner ohne Computerunterstützung, d.h. klassisch analog lesend und hörend verarbeiten kann. Welch scharfer Kontrast zu den zwei Handvoll Werken, die unverdrossen das Kernrepertoire bilden.

Über diese Indie-Grass-Roots-Bewegung als solche weiß man noch nicht viel. Es fehlt, soweit ich jedenfalls sehe, noch jede systematische Forschung. Über ihre Ökonomie und ihre Motivation, ihr Netzwerk und ihre Resonanz, worüber bis dato vor allem gelegentliche Interviews, Medienberichte und Diskussionen in sozialen Medien, Foren und Blogs etwas andeuten. Diese dezentral entstandene und organisierte Indie-Grass-RootsBewegung besser beschreiben zu können, ist ebenfalls Teil des Programms des eingangs erwähnten laufenden Pilotprojekts an der Deutschen Nationalbibliothek. An dieser Indie-Grass-Roots-Bewegung beteiligen sich zahllose kleine und kleinste Plattenfirmen, Musikverlage und Musiker, Blogger, Autoren von Wikipediaartikeln und Liebhaberseiten usw., bis in die Amateurkultur hinein (deswegen `Grass Roots $`$ ). Massiv befördert durch das Internet und sein Potential, unmittelbar Öffentlichkeit herstellen zu können, hat sich hier in der Zwischenzeit potenziert, was Anselm Gerhard schon zur Jahrtausendwende als Trend wahrnahm:

»Dass es gerade um die letztgenannte Eigenschaft [Neugierde ist gemeint, Anm. d. A.] in der akademischen Musikforschung nicht (mehr) zum Besten bestellt ist, zeigt der Umstand, dass inzwischen [...] weit mehr Entdeckungen durch forschende Interpreten auf Tonträgern präsentiert werden als in den Publikationsorganen der musikhistorischen Forschung selbst. $\ll^{86}$ 
Man muss nur auf die Plattenfirmen, Künstlernamen und Veröffentlichungsdaten in den diskographischen Angaben des Schlusskapitels schauen, um hiesige Diagnose bestätigt zu finden. Inzwischen ist ein signifikanter Teil des über die Jahrzehnte enorm angewachsenen Repertoires in gedruckten Noten greifbar. Mehr noch sind über diese Indie-Grass-RootsBewegung mittlerweile Klavierquintette in dreistelliger Zahl als Tonträger allein schon über die üblichen Handelshäuser wie Amazon und jpc oder über iTunes unschwer erhältlich oder über allgemeine Streamingportale wie YouTube und Spotify oder speziell auf Klassische Musik ausgelegte wie die Naxos Music Library oder Idagio abrufbar. Zahlreiche, nie auf Tonträger veröffentlichte Klavierquintette kommen in Liveaufnahmen hinzu, zugänglich über das Internet. Während in den 1990er Jahren meine Liste der Stücke in diesem Genre, die ich gerne einmal hören wollte, ausgesprochen lang war, sind nun nicht mehr allzu viele verblieben, bei denen ich in der Zwischenzeit nicht auf die eine oder andere Weise Gelegenheit dazu hatte, weil nirgendwo im Netz eine Studioaufnahme oder mitgeschnittene Liveaufführung zu finden war. Wo ich in den 1990er Jahren vor dem allgemeinen Zugriff auf das Internet über die meisten Werke in meist schon ziemlich vergilbten Büchern kaum mehr als einen Namen finden konnte, oft versteckt in irgendwelchen Nebensätzen, Fußnoten oder gar Chroniken und Listen, habe ich heute vielfach mit ein paar Klicks Noten und Tonaufnahmen verfügbar. Mitunter nicht in der Qualität kritischer wissenschaftlicher Ausgaben oder der Einspielungen der besten Ensembles auf den renommierten Labels, in exzellenten Studios oder Konzertsälen von führenden Produzenten aufgenommen. Aber doch regelmäßig gut genug. Und nicht selten sogar hervorragend. Viele Werke, oft als Erstveröffentlichungen, mitunter als reine Netzpublikationen, erreichen mittlerweile über diese Indie-Grass-Roots-Bewegung auch den regulären Tonträger- und Notenmarkt. Auf seine nerdig-verquere Art in den Größenordnungen, die Kammermusik überhaupt möglich sind, boomt dieses Genre also. Nur tut es dies eben konsequent abseits des Mainstreams Klassischer Musik (deswegen $>$ Indie $<$ ). Der sich nicht bewegt.

All diese Musik hören zu können, erlaubt es einem nun, sich selbst ein Urteil zu bilden, ob der skizzierte rigide Status quo sich in einer nachvollziehbaren Weise einer offensichtlich geringeren Qualität derjenigen Werke und Akteure verdankt, die einem in diesem Genre abseits der wenigen Markennamen in Komponist und Interpret, Aufführungsort und Label be- 
gegnet. Für mich jedenfalls ist das Klavierquintett keineswegs die 3. Liga der Klassischen Musik, beschenkt nur mit wenigen Lichtblicken. Hier geht es nicht um Schundliteratur, leere Kommerzware oder handwerklich dilettantische Amateurkunst. Wer sich Zutritt verschafft zu diesem Genre abseits des Mainstreams Klassischer Musik merkt schnell: Das Gegenteil ist der Fall. Mir jedenfalls ging es so. Das Missverhältnis zwischen Qualität und Aufmerksamkeit erscheint mir geradezu eklatant. Jedenfalls im heutigen Musikbetrieb, um den er mir hier geht. Ich jedenfalls erfahre das Klavierquintett als ein Genre mit einer hohen Dichte an hörenswerter Musik, wie im letzten Teil des Buchs eingehend verhandelt werden wird. Einer Dichte, die in keinem Verhältnis steht zur extrem zugespitzten markennamengetriebenen Aufmerksamkeitsökonomie in den Leitmedien heutiger Klassischer Musik.

Wie immer man das auch sieht: Die Entwicklung besagter Indie-GrassRoots-Bewegung im Bereich des Klavierquintetts ist jedenfalls ein Faktum. Und gehört zu einem weit größeren Trend. Bereits 1985 wies Joseph Kerman in Contemplating Music: Challenges to Musicology, seiner klassischen Studie zur Verfasstheit der Musikwissenschaft, auf einen Umstand hin, der ihm für die Musik der Gegenwart besonders wesentlich erschien - das Anwachsen der zum Hören verfügbaren Musik:

\begin{abstract}
»But the crucial thing, I believe, was that now listeners could and did obtain great masses of music of all kinds and were able to browse through it on recordings, in something like the way they were used to browsing through literature of all kinds in books. Previously only professional musicians had been able to move around in music with such (actually less) flexibility by reading scores. The range and sheer amount of music known to non-musicians and musicians alike went up exponentially; musical composition, musical performance, and musical consumption were all affected by the electronic revolution of the 1950s, but consumption was affected most. $\ll^{87}$
\end{abstract}

Das quantitative Ausmaß dieses Anwachsens seit Thomas Alva Edisons Erfindung der Tonaufnahme gut ein Jahrhundert zuvor im Jahre 1877 kann man sich nicht deutlich genug vor Augen halten, wie Anselm Gerhard 15 Jahre nach Kerman anmerkte: 
»seit der Begründung der modernen Musikgeschichtsschreibung durch Guido Adler [sind] mehr als 100 Jahre vergangen, und über Kataloge, Bibliografien, Editionen und Tonträger haben wir leichtesten Zugang zum Mehrhundertfachen an historischer Musik als unsere Urgroßeltern [...]...18

Die Dynamik dieser Entwicklung hat sich in den vergangenen Jahren durch das Internet nochmals geradezu exponentiell potenziert. Allein hierzulande stehen derzeit Hörern fast 2.380.000 digitaler Alben und gut 280.000 physische Alben auf Tonträgern zur Verfügung, bei gut 20.000 Neuerscheinungen jährlich, davon 20-25 \% im Bereich Klassischer Musik. ${ }^{89}$ Der Schwerpunkt liegt dabei digital inzwischen auf Streaming, wo Anbieter wie Apple, Pandora und Spotify riesige Musikbibliotheken von zwischen 35 und 45 Millionen Einzeltracks anbieten - von YouTube und SoundCloud ganz zu schweigen. ${ }^{90}$ Noch nie war so viel Musik zu haben. Nicht nur in der kleinen Genrewelt des Klavierquintetts. Selbst das Gesamtangebot physischer Tonträger hat sich seit Gerhards Kommentar zur Jahrtausendwende binnen einer Generation nochmals verdoppelt. ${ }^{91}$ Es ist eine »alexandrinische Bibliothek der Musik «"22 (Peter Gülke) entstanden.

Dabei ist für einen Zugang zu dieser >Bibliothek` meist keine aufwendige Anreise mehr erforderlich. Oder eine nennenswerte Investition. In weiten Teilen steht sie einem stattdessen mit nur wenigen Klicks zum Anhören zur Verfügung. Vieles für mittlerweile sehr geringe Anschaffungsoder Abonnementgebühren. Oder gleich ganz umsonst. Nach dem Tauschbörsenboom der Jahrtausendwende inzwischen sogar vielfach ganz legal frei zugänglich. Werbefinanziert. Oder als historische, rechtefreie Tondokumente. Oder weil die Künstler es von sich aus kostenlos ins Netz stellen. Für jemanden wie mich, der Musik liebt, der ganz unterschiedliche Arten von Musik hört, der dem Entdecken und Erkunden neuer Musik mit Leidenschaft nachgeht, aber in den Mühen und engen Grenzen der vordigitalen Musiksuche in der norddeutschen Provinz aufgewachsen und sozialisiert wurde, ist all dies schlicht ein Traum.

Nur: »She speaks, yet she says nothing: what of that?«, wie sich Romeo beim Anblick von Julia zu Beginn der Balkonszene von William Shakespeares gleichnamigem Drama fragt. ${ }^{93}$ Ein klassisches Beispiel für einen traumhaften Moment, einen Moment wie im Traum - bevor die Protagonisten die Konsequenzen ihres Traums mit voller Wucht trifft, als sie versuchen, den Traum zu leben, mit Sinn zu erfüllen. 
What of that? Die Frage an dieser Stelle ist also nicht, wie man sseine Julia findet . Eine zentrale Frage. Aber nicht die, die ich verfolgen werde. D.h. in meinem Fall hier nicht, wie man in dieser Überfülle zu Musik kommt, die für einen von Belang ist. Einen mit der Intensität angeht wie Julia Romeo. Intellektuell, funktional, emotional - weswegen auch immer man nach Musik sucht, wonach auch immer in Musik. Das Gesuchte möglicherweise sogar in Kontexten entdeckt, in denen man es gar nicht erwartet. ${ }^{94}$ Vielleicht gar nicht danach gesucht hat. Weil man nichts davon ahnte. Oder in der Überfülle nicht wusste, wo man zu suchen anfangen soll. Oder man nie auch nur daran gedacht hätte, dass es an der einen oder anderen Stelle überhaupt etwas für einen zu finden geben könnte. Wider den eigenen Vorurteilen etwa. So wie Romeo Julia auf einer Feier der Erzfeinde seiner eigenen Familie begegnet. Of all places.

All das sind Themen, die mich in meinem Umgang mit Musik sehr beschäftigen. Und enorme Herausforderungen für die Erschließung besagter alexandrinischer Bibliothek bergen. Für die Orientierung in ihr. Für ihre Lebendigkeit. Für all die Algorithmen und Playlists etwa, deren Stärke bislang doch vor allem darin liegt, passende, aber regelmäßig eben erwartbare Ergebnisse zu produzieren, über Ähnlichkeiten etwa. ${ }^{95}$

Aber die Analogie zu Romeo und Julia, das Shakespeare-Zitat, die Frage What of that? - all das zielt auf etwas anderes, das mich gleichermaßen umtreibt und dem ich hier nachgehen werde: Wie soll man für sich Sinn ziehen aus dieser unüberschaubaren, undurchdringlich wirkenden Fülle an stetig neu erschlossener und neu entstehender Musikgeschichte? Schon im Fall des Klavierquintetts mittlerweile in der erreichten Erschließungsquote an Noten, Aufnahmen, Kommentaren usw. für einen Einzelnen mit den manuellen Mitteln des Hörens, Spielens und Lesens eigentlich nicht mehr $\mathrm{zu}$ beherrschen? Bleibt einem heutzutage überhaupt noch etwas anderes, um Orientierung, ja überhaupt eine eigene Meinung über eine Sache wie ein solches Genres zu gewinnen, als sich der Herrschaft und Führung durch Algorithmen und Statistiken zu überlassen? Getreu Gerhard Lauers Prognose:

»Die schiere Datenmenge solcher und ähnlicher Vorhaben braucht den Computer. [...] Die aktuelle Transformation der Geisteswissenschaften betrifft weniger die Tiefe, in der Kultur mithilfe neuer Ansätze erschlossen werden kann, sondern die Breite der entsprechenden Unterfangen. Im analogen Zeitalter waren 
kulturellen Beständen enge Grenzen gesetzt, weshalb man sich auf einen abgeschlossenen Kanon konzentrieren musste. Das digitale Zeitalter macht nun (fast) die gesamte Kultur zugänglich. Kulturelle Evolution statt Geschichte - so könnte man zugespitzt die Richtung umschreiben, welche die geisteswissenschaftliche Forschung in den nächsten Jahren bestimmen wird. $\ll^{96}$

Versperrt einem also der >Traum dieser vor einer Generation nicht absehbaren Erschließung des Klavierquintettgenres, die einem Musikhistoriker nun die einst ebenso plausible wie gängige Entschuldigung nimmt, den Rest abseits der Kernrepertoires nicht kennen und entsprechend beachten zu können, jede andere Form, Musikgeschichte adäquat und produktiv zu erzählen, mit Sinn zu erfüllen, als mit den großen Datenmengen zugewandten Analysezielen und -methoden der Digital Humanities? Jenem derzeit politisch so stark geförderten Zugriff auf das Feld der Geisteswissenschaften, für den ich eben thematisch verantwortlich bin im Leitungsstab der Generaldirektion eben einer der auch für die Musik zentralen Gedächtnisinstitutionen und Kulturerbeeinrichtungen dieses Landes, der Deutschen Nationalbibliothek. In dessen Diskurse ich entsprechend vertieft bin. Und mit dessen Entwicklungen und Herausforderungen ich ringe. ${ }^{97}$

Digital Humanities ist leicht gesagt. Die überbordende Dynamik der Digital Humanities dieser Tage geht aber bei genauem Hinsehen einher mit einer großen Unsicherheit über ihre Verfasstheit, ihren Auftrag und ihre Leistungsfähigkeit. ${ }^{98}$ Hier lohnt also ein kurzes Innehalten. Denn schon die

»Gretchenfrage, ob es sich bei den Digital Humanities um eine Disziplin, eine Sammlung an Methoden oder eine in den jeweiligen [geisteswissenschaftlichen] Disziplinen verankerte Grundwissenschaft handelt «, ${ }^{99}$

wie Mareike König erläutert, d.h. u.a. eben, ob man im Fall der Digital Humanities Infrastrukturfragen und Forschungsinhalte, hilfswissenschaftlichen Auftrag und eigenständig-disziplinären theoretisch-methodischen Anspruch sauber trennen kann oder gar sollte, ist bis in die Digital Humanities hinein weithin umstritten. ${ }^{100}$ Definitionsversuche sind ein Genre für sich. ${ }^{101}$ Aber ebenso Witze und Äußerungen des Unbehagens über die Schwierigkeit, genau dies nicht präzise leisten zu können. ${ }^{102}$ Diese ungebrochene Verunsicherung darüber, was Digital Humanities sind und in welchem Verhältnis traditionelle Geisteswissenschaften wie die Musikwissenschaft 
dazu stehen, d.h. womit man es beim Digital Turn eigentlich zu tun hat, hat vor allem drei Gründe:

Erstens ist das Feld viel älter als sein heutiger Name Digital Humanities. ${ }^{103}$ Die Bezeichnung etablierte sich erst im Gefolge eines für dieses Feld und seine jüngere Entwicklung grundlegenden Sammelbandes, des Companion to Digital Humanities von Susan Schreibman, Ray Siemens und John Unsworth aus dem Jahr 2004. ${ }^{104}$ Heute überstrahlt der Begriff als geradezu omnipräsentes Buzzword, als der nächste große Turn, den Wissenschaftsdiskurs geisteswissenschaftsübergreifend geradezu - oder überschattet ihn, je nach Sichtweise und Position. Die Association for Literary and Linguistic Computing (ALLC), seit 2012 die European Association of Digital Humanities [EADH], hatte sich als europäischer Zentralverband freilich bereits 1973 gegründet, die amerikanische Association for Computers and the Humanities (ACH) war nur fünf Jahre später gefolgt. Die einschlägige IMS Study Group, die heute Digital Musicology heißt, stammt immerhin auch schon aus dem Jahr $1987 .{ }^{105}$ Da war das erste Fachjournal des Feldes, Computers and the Humanities (1966), bereits in seinem 21. Erscheinungsjahr, genauso alt, wie die ersten Konferenzreihen und universitären Forschungszentren. Auch Fachbegriffe wie »Computational Musicology« und »Music Information Retrieval« erscheinen bereits in den 1960er Jahren in der Literatur. ${ }^{106}$ Die konzeptuellen Anfänge des traditionell als Gründungsprojekt computergestützter Geisteswissenschaft angeführten Index Thomisticus zu den Schriften Thomas von Aquins, erarbeitet von Roberto Busa, hatten sogar bereits in der Mitte der 1940er Jahre gelegen und waren schon 1949 in eine langanhaltende Kooperation mit IBM überführt worden. D.h., dass sich heute unter dem Lemma Digital Humanities vieles wiederfindet, das früher anders hieß bzw. eingeordnet war.

Zweitens ist das Feld von einer großen, aber einigermaßen ungeordneten Dynamik geprägt, gerade in Deutschland, wo die Entwicklung eigentlich erst zu Beginn des noch laufenden Jahrzehnts so richtig an Fahrt aufgenommen hat. ${ }^{107}$ Digital Humanities sind derzeit eine akademische »growth industry«. ${ }^{108}$ Diese Dynamik der Digital Humanities, international wie hierzulande, zeichnet sich an vielen unschwer von außen erkennbaren Entwicklungen ab. Das BMBF hat z.B. die Digital Humanities im August 2018 zur eigenständigen akademischen Disziplin ausgerufen. ${ }^{109}$ In den vergangenen zehn Jahren wurden allein im deutschsprachigen Hochschulbereich gut 60 Digital-Humanities-Professuren besetzt. ${ }^{110}$ Der Mittelbauar- 
beitsmarkt ist ebenfalls ausgesprochen lebendig. Digital-Humanities-Kompetenzen werden jedenfalls als Softskill inzwischen in geisteswissenschaftlichen Stellenausschreibungen regelmäßig nachgefragt. ${ }^{111}$ Das war zuletzt von München bis Kiel auch in der Musikwissenschaft zu beobachten. Der Schwerpunkt auf Digital Humanities im Bereich der Drittmittelförderung ist ebenfalls nicht mehr zu ignorieren. ${ }^{112}$ Ebenso wenig wie der Umstand, dass Digital-Humanities-Projektstrategien inzwischen auch formal zu Antragsvoraussetzungen bei Drittmittelverfahren gemacht werden, etwa hinsichtlich der Nachnutzbarkeit von Daten. ${ }^{113}$ Bis 2011 gab es überhaupt nur zwei grundständige Studienprogramme, die Digital Humanities im Titel führten - inzwischen eröffnen jedes Wintersemester drei bis vier neue. ${ }^{114}$ Lange Zeit gab es nur wenige universitäre Kompetenzzentren wie GCDH (Göttingen), $\mathrm{CCeH}$ (Cologne), Trier Center for Digital Humanities (Trier) und Austrian Centre for Digital Humanities (Graz). ${ }^{115}$ Inzwischen kommen ständig neue hinzu, wie in Hamburg, Heidelberg, Mainz, Münster, Stuttgart oder Würzburg. ${ }^{116}$ Gleichzeitig werden regionale Netzwerke erprobt, wie der ifDHb in Berlin/Brandenburg und die AG Digital Humanities Mitteldeutschland in Mitteldeutschland. ${ }^{117}$ Die traditionellen Fachgesellschaften der Geisteswissenschaften etablieren ebenfalls Working Groups, so wie neben der Fachgruppe Digitale Musikwissenschaft der Gesellschaft für Musikforschung (2016) zuletzt z.B. auch die Amerikanistik, Germanistik, Geschichtswissenschaft, Kunstgeschichte, Mediävistik, Romanistik und Semiotik. ${ }^{118}$ Der europäische Dachverband der Akademien ALLEA hat eine E-Humanities Working Group, die Union der deutschen Akademien ebenso wie z.B. das europäische Pendant im Bibliothekswesen, LIBER, oder der deutsche Bibliothekarsverband VDB. ${ }^{119}$ Inzwischen fällt als geisteswissenschaftliche Institution eher auf, wer keine Digital-Humanities-AG hat. Darüber hinaus wurden neue Fachzeitschriften wie Digital Humanities Quarterly (2007), Digital Studies/Le Champ Numérique (2008), Journal of Digital Humanities (2011-2014), DH Commons (2015) und Zeitschrift für digitale Geisteswissenschaften (2015) etabliert bzw. im Fall des führenden Field Journals von Literary and Linguistic Computing (1986) zu Digital Scholarship in the Humanities (2015) umbenannt, was gleichfalls die geisteswissenschaftlich übergreifende Expansion des Feldes markiert. ${ }^{120}$ Überhaupt ist die Publikationsdichte sehr hoch. ${ }^{121}$ All das heißt natürlich, dass derzeit in großer Zahl aus unterschiedlichster Richtung neue Akteure mit verschiedensten Hintergründen und Zielen zum Feld dazustoßen oder in- 
nerhalb dessen ihre Position modifizieren. Die skizzierte Dynamik befördert nicht nur das aktuelle Momentum der Digital Humanities, sondern zugleich natürlich auch die Heterogenität des Feldes. Zum Beleg hierfür muss man sich nur anschauen, an wie vielen verschiedenen Fakultäten die besagten neuen Digital-Humanities-Professuren und -Studienprogramme angebunden sind und wie verschieden ihre Curricula gestaltet sind.

Drittens schließlich wirkt der Begriff wie ein Schwamm. Praktisch jede Betätigung in den Geisteswissenschaften, die mit einem Computer zu tun haben, findet sich aufgesogen und als Teil in die Digital Humanities eingemeindet. Da kaum noch etwas in den Geisteswissenschaften ohne Computer vonstatten geht, ist das daraus entstehende Problem unvermeidlich: Digital Humanities ist zu einem ausnehmend heterogenen Sammelbegriff geworden für unterschiedlichste Aktivitäten im Bereich digitaler Geisteswissenschaft. ${ }^{122}$ Dazu gehören die wichtigen und oftmals ganz praktisch sehr nützlichen, aber eben klar hilfswissenschaftlichen Bereiche der Digitalisierung und Aufbereitung nicht-digitaler Daten wie Handschriften (insb. Fragen der Automatisierung der Erfassung nicht-digitaler Daten und der Fehlerreduktion hierbei), der Strategien zur Erfassung und Erhaltung originär digitalen Kulturerbes (einschließlich des Diskurses, was digitales Kulturerbe ist), der technischen und inhaltlichen Standards für digitale Daten, der Langzeitarchivierung und Langzeitnachnutzbarkeit (digitalisierter und originär) digitaler Daten, des Zugangs zu digitalen Daten (einschließlich Fragen des Designs von Suchmaschinen, des Urheberrechts, des Datenschutzes und der Forschungsethik), der Verknüpfung digitaler Datenbestände, der digitalen Philologie und Editionswissenschaft, der Möglichkeiten des Text und Data Mining (TDM), des digitalen Veröffentlichens (einschließlich Open Access), der Weiterentwicklung von Evaluationsstandards für Forschung unter digitalen Bedingungen (einschließlich neuer Publikationsformen), der computergestützten Visualisierung von Forschungsresultaten, des digitalen, crossmedialen Storytellings, der Öffnung von Forschung für Nichtwissenschaftler (Crowdfunding, Crowdsourcing, Wikitools etc.), der Auseinandersetzung mit dem Einfluss digitaler Rahmenbedingungen wie User Interfaces auf die Wahrnehmung und Erschließung von Daten einschließlich des Einbringens in die Weiterentwicklung von Computerhard- und -software sowie schließlich die Weiterentwicklung der digitalen Lehre. In diesem weiten Sinne betreibt letztlich jeder Musikwissenschaftler, der für seine Arbeit einen Computer verwendet, und sei es auch 
nur für die Recherche oder die Präsentation seiner Forschungsresultate oder Lehrinhalte, zumindest bis zu einem gewissen Grad Digital Humanities. Die Sache wird dadurch weiter verkompliziert, dass Infrastrukturentscheidungen in diesem Kontext immer wieder inhaltliche Implikationen aufweisen und hiervon nicht sauber zu trennen sind. ${ }^{123}$ Sogar noch herausfordernder ist das vielfach formulierte Versprechen der Digital Humanities, sneue Fragestellungen, Methoden und Antworten bereitzuhalten. ${ }^{124}$ Mitunter wird sogar der Maximalanspruch formuliert, insgesamt »das gesellschaftliche Wissen neu zu organisieren « und zugleich »eine paradigmatisch alternative Form der Wissensgenerierung darzustellen ${ }^{125}$, wie Ramón Reichert referiert.

Als Begriff und Feld lassen sich die Digital Humanities in Verfasstheit, Auftrag und Leistungsfähigkeit derzeit also aus vielerlei Gründen nicht klar bestimmen. Das gilt erst recht im Fall eines anders als bei Literaturwissenschaft oder Archäologie spät hinzustoßenden Gegenstandsbereichs wie jenem der Historischen Musikwissenschaft. Wo bislang also vergleichsweise wenig passiert ist, abseits des Kerngeschäfts von Editionsprojekten. ${ }^{126}$ Doch ungeachtet all dieser Dynamiken und Unsicherheiten, trotz all der offenen Fragen zum Vorgehen: Erscheint der Rekurs auf die Digital Humanities aufgrund ihres Griffs nach großen Datenmengen nicht letztlich dennoch bereits jetzt geradezu alternativlos, so muss man fragen, will man heutzutage Musikgeschichte adäquat und produktiv erzählen, mit Sinn erfüllen? Eine Frage, die sich nicht nur aufdrängt angesichts der genannten, einen unvermeidlich überfordernden Zahlen zur nun in Schrift, Ton und Bewegtbild verfügbaren Musik. Sondern auch in Anbetracht der skizzierten ungebrochen dominierenden Art und Weise, wie Musikgeschichte in den Leitmedien Klassischer Musik konstruiert und tradiert wird. Die so aus der Zeit gefallen ist in der Enge ihres Zugriffs auf Musik. Wo es geschafft wird, aus einer mehr als zwei Jahrhunderte langen Produktion von Klavierquintetten lediglich einem guten Dutzend Arbeiten überhaupt nennenswert Aufmerksamkeit zu schenken. Während das Genre über einen Bestand an Werken im vierstelligen Bereich verfügt. Müsste man nicht all diese Musik erst einmal in den Griff bekommen, bevor man etwas über das Genre als Genre sagen, gar eine Geschichte und ein Gegenwartsportrait dieses Genres schreiben kann? Denn was ist das überhaupt für Musik? Und was ist das für eine musikalische Praxis darum herum, von Komponisten bis Interpreten, von Verlagen bis Labels, in der Vergangenheit wie heute? 
Angesichts dieses Fallbeispiels in Richtung Digital Humanities zu denken, drängt sich also schon deswegen auf, weil das Genre des Klavierquintetts als geradezu paradigmatischer Gegenstand erscheint, für die Musik einmal jenes große Versprechen und Potential der Digital Humanities zu erkunden, das Fotis Jannidis für die Literaturwissenschaft so treffend beschrieben hat, das aber gleichermaßen für die Musikwissenschaft gilt:

»Formelhaft könnte man davon sprechen, dass die traditionelle Literaturwissenschaft sehr viel über sehr wenige Texte weiß, die digitale Literaturwissenschaft dagegen sehr wenig über sehr viele Texte. Literaturgeschichte kann auf diese Weise z.B. den dunklen Kontinent der einstmals gelesenen, heute aber weitgehend unbekannten Texte wenigstens in Grundzügen erschließen.« ${ }^{127}$

Schon auf den ersten Blick erscheint das Genre des Klavierquintetts in den Leitmedien Klassischer Musik als genau so ein >dunkler Kontinent<. Geradezu als Inkarnation dessen angesichts der Enge des Kernrepertoires. Über dies als ein Musterbeispiel, das sich aufgrund seiner Spezifik als Genre den üblichen Mechanismen zur Rechtfertigung der hinter dieser Dunkelheit stehenden, konzentrierten historiographischen Auswahl entzieht, von der Heroen- über die Gattungs- bis zur Einflussmusikgeschichtsschreibung. Schon alleine deswegen, weil sich eine Annäherung an Geschichte und Gegenwart dieses Genres in Meistern und genredefinierenden Meister- und Referenzwerken eben nicht sinnvoll finden lässt. Weil aus historischem Zufall die Markennamen Klassischer Musik fast vollständig fehlen.

Sich dem Klavierquintett in dieser Weise als Fallbeispiel für digitale Musikwissenschaft anzunähern, mehr Licht ins historiographische Dunkel dieses Genres zu bringen, ist genau deswegen eines der Anliegen meines derzeit laufenden Pilotprojekts an der Deutschen Nationalbibliothek. Dessen Ergebnisse werden dereinst Thema an anderer Stelle werden. Hier bedarf es noch viel Entwicklungsarbeit. Schon auf der Ausgangsebene der Erstellung auswertbarer vernetzter Datenkorpora. ${ }^{128}$ Hierfür entscheidende Rahmenbedingungen wie das Urheberrecht (vgl. nur $\S 60 d$ UrhG und Art. 3 der EU-Richtlinie über das Urheberrecht im digitalen Binnenmarkt) oder das nun von Bund und Bundesländern aufgelegte Programm zum Aufbau von Nationalen Forschungsdateninfrastrukturen (NFDI) haben überhaupt gerade erst begonnen, sich in dafür erforderlicher Weise zu ändern. 
Im Folgenden werde ich das Pferd jedoch von der anderen Seite aufzäumen. Den Blick schon einmal auf jene genau in diesem Zusammenhang auftretende, aber bereits jetzt adressierbare Vorfrage zu richten: Jene nach der ästhetischen Qualität. So wie sie sich u.a. eben in meinem eigenen ästhetischen Erleben des Genres zeigt. Ein für den angestrebten Versuchsaufbau essentieller Schritt. Was soll ich damit anfangen? Soll ich überhaupt etwas damit anfangen? Das ist etwas, das von vornherein mitbedacht werden muss. Denn verantwortlich für eben jene Digital Humanities bei der Deutschen Nationalbibliothek, ringe ich derzeit eben nicht nur in institutioneller und forschungspolitischer Hinsicht mit all den angeführten Herausforderungen. Vielmehr noch stehe ich in Auseinandersetzung mit den Potentialen und Grenzen, Versprechen und Risiken, Erfolgen und Enttäuschungen, Best-Practice-Beispielen und versandten Einzelprojekten digitaler Geisteswissenschaften. Und eine der Fragen, die ich mir in diesem Kontext stelle, ist quasi die ultimative Gegenfrage schlechthin. Jene nach der Reichweite eines solch digitalen, großdatengetriebenen Forschungsansatzes. Lohnt die Mühe überhaupt? Worauf lässt sich hoffen, dass auf diesem Wege erreicht werden kann? Und was vor allem erscheint unerreichbar, egal, wie gut die Datenkorpora, gleichgültig, wie aussagekräftig die Analysemethoden werden? Unerreichbar, aber eben nicht vernachlässigbar? Wie hier, so ist die Frage, eben das Unbehagen über die vorgefundene Situation des Genres in den Leitmedien Klassischer Musik im Abgleich mit meinem eigenen ästhetischen Erleben dieser musikalischen Praxis?

Das ist die Vorfrage, um die es mir hier geht. Wie sähe also eine $\mathrm{Mu}-$ sikgeschichte des Klavierquintetts aus, die nicht den großen Datenmengen zugewandten Analysezielen und -methoden der Digital Humanities folgt? Als radikaler Gegenentwurf quasi zu Gerhard Lauers Postulat beim Akademientag 2018: »Die Zukunft der Geisteswissenschaften ist die Mathematik «? ${ }^{129}$ Als radikaler Gegenentwurf aber auch zu meinem eignen Pilotprojekt? Lohnt es, einen solchen Gegenentwurf überhaupt zu denken? Gar dafür zu streiten? Als Korrektiv? Als Ergänzung? Als gleichfalls unverzichtbarer Teil eines historiographisch zu schaffenden Gesamteindrucks von der Sache, dem Genre des Klavierquintetts also? Ist das zu tun vielleicht sogar zwingend notwendig? Erst recht angesichts der überbordenden Dynamik und Reichweite der Versprechen, die derzeit in den Digital Humanities stecken? 
Denn was ist, so ließe sich im Angesicht von Lauers zugespitztem Postulat zurückfragen, wenn es einem als Musikhistoriker einmal nicht um das Ermitteln von und Berichten über Korrelationen und Muster, Daten und Zahlen, Fakten und Tatsachen, Standards und Normen, Statistiken und Kanons geht. Sondern um das, was Bruno Latour schlagend »matters of concern, Dinge, die uns angehen oder Dinge von Belang « genannt hat? ${ }^{130}$ Musik etwa, für die eine Leidenschaft besteht. Die für einen zählt. Die einen erreicht. Für die einem aber völlig egal ist, ob sie statistisch wichtig oder repräsentativ ist, ob sie soziales Prestige hat oder historische Relevanz, wissenschaftlichen Wert, politische Bedeutung oder ökonomische Signifikanz. Hinsichtlich derer einem gleichgültig ist, ob viele sie mögen oder man sich ganz allein damit fühlt. Ob sie zum Kanon gehört oder nicht. Oder all diese Ebenen durcheinander gehen und einander abwechseln, von Track zu Track. Etwa in einem Ordner auf dem eigenen iPod. Den man ganz für sich zusammenstellt und anhört. Als gelebte, persönlich kuratierte Musikgeschichte quasi.

Vom eigenen ästhetischen Erleben von Musik auszugehen, erscheint mir nicht nur mit Barbara Flückiger einen Grenzkonflikt zwischen traditionell-hermeneutischer und digital-empirischer Geisteswissenschaft aufzurufen. Sondern eine maximale Gegenposition zu formulieren. Sowohl zu den Digital Humanities als im Übrigen auch, wie noch zu sehen sein wird, zur herkömmlichen Historischen Musikwissenschaft. Die radikalste Basis also, um einen Gegenentwurf zu denken. D.h. dort anzufangen, wo einen selbst eine Musik emotional erreicht. Beim Nachdenken über die selbst kuratierte Musikgeschichte. Nicht, um die ästhetischen Wertungen Anderer heute oder gar in der Vergangenheit an den eigenen gegenwärtigen Erfahrungen und Maßstäben zu messen - ein in der Musikgeschichtsschreibung verbreitetes, aber deprimierend ahistorisches Unternehmen, wie Frank Hentschel anmahnt. ${ }^{131}$ Sondern weil empirische Annäherungen jeder Art, von physiologischen und neurologischen Messdaten über Befragungsstatistiken bis hin zu Verkaufs- und Klickzahlen, die Reflexion über das eigene ästhetische Erleben und dessen Verortung in den musikalischen Bezugspunkten anreichern, informieren und herausfordern, aber als Instanz eben nicht ersetzen können. Und deren Relevanz schlicht zu leugnen, wie immer wieder allerorts in musikwissenschaftlicher Literatur zu lesen, widerspricht zumindest meinem eigenen Umgang mit Musikgeschichte, meinem Bewegen in Mu- 
sikgeschichte. Das setzt sich in meinem Ringen um die Möglichkeiten und Grenzen der Digital Humanities fort. Hierfür möchte ich streiten.

$\mathrm{Ob}$ Geisteswissenschaft das eigene ästhetische Erleben und die damit einhergehenden Werturteile überhaupt berücksichtigen, geschweige denn ihnen historiographisch folgen darf, ist nicht nur in der Musikwissenschaft bekanntlich heiß umstritten. ${ }^{132}$ Von der Perspektive der Digital Humanities aus gesehen muss es abgelehnt werden, wie Lauers Aussage impliziert. ${ }^{133}$ So wie es zuvor schon von Geschichts- und Kulturwissenschaft, Philosophie, Psychologie und Soziologie abgelehnt wurde, wenn sie von ihrer jeweiligen disziplinären Position aus die Musik in den Blick nahmen. Aber mich irritiert mein Unbehagen, wenn ich Aussagen wie jene von Lauer höre. Und das gerade, weil ich viel wissenschaftlich ausgesprochen ertragreich finde, was unter dem Lemma Digital Humanities erarbeitet wird - so wie es mir schon mit den Ansätzen und Erkenntnisinteressen gegenüber Musik von Geschichts- und Kulturwissenschaft, Philosophie, Psychologie und Soziologie ging. Derzeit wie angesprochen etwa im Blick darauf, eine Vorstellung von den heute potentiell zum Hören verfügbaren Musikmassen zu gewinnen. Nicht nur der Musik überhaupt, den Abermillionen nichtgehörten Tracks bei Spotify, YouTube und SoundCloud etwa - eine mich sehr interessierende Frage. Sondern schon beim Klavierquintett, wo das inzwischen erreichbare Material beginnt, sich meinen manuellen Erschließungskapazitäten zu entziehen. Um z.B. die außerordentliche musikalische Produktivität der Gegenwart nachvollziehen zu können. Etwa die besagte Indie-Grass-Roots-Bewegung. Und wie soll dies anders gehen als mittels algorithmischer Analyse, will man sich eben nicht auf die Position einer über Markennamen vorgerechtfertigten Auswahl zurückziehen?

Für mich bleibt aber bei alledem, beim Ruf nach dem Primat der Mathematik, der algorithmischen Erschließung großer Datenmengen, eine besonders deutlich spürbare und daher zu adressierende Leerstelle zurück. Und zwar eben genau dann, wenn man dergleichen >matters of concern`, wie sie Latour im Blick hat, nicht nur zufällig finden will, sondern man in Musikgeschichte aktiv nach ihnen sucht und zugleich anderen als Musikgeschichte zeigen will in dem Sinne, den Ben Ratliff in seiner Studie Every Song Ever, Twenty Ways to Listen to Music Now als Fragen aufgeworfen hat: 
»Now we can hear nearly everything, almost whenever, almost wherever, often for free: most of the history of Western music and a lot of the rest. We know all that music is there. Some of us know, roughly, how to encounter a lot of it. But once we hear it, how can we allow ourselves to make sense of it? [...] The most significant progress in the recent history of music has to do with listening. How we listen to music could be, for perhaps the first time in centuries, every bit as important to its history and evolution [...]. How are we going to access it, and how can we listen to it with purpose - meaning, how can we pay just enough attention to it so that it could change our lives? «134

Auf eben diese Fragen steuert diese kleine Abhandlung im Folgenden zu. Auf Ratliffs »purpose«. Um für mich selbst einmal zu klären, welche Art Musikgeschichte ich eigentlich wirklich schreiben und lesen will. Keineswegs immer. Und erst recht nicht gegen die Digital Humanities. Aber in Ergänzung dazu. Im Zusammenspiel damit. Vor dem Hintergrund der Auseinandersetzung mit der dortigen Perspektive eben doch immer dann, wenn mir an >matters of concern $<$ gelegen ist. Meinen >matters of concern ২. Und die Frage in den Raum tritt: What matters in >matters of concern`, was geht einen an bei Dingen von Belang? Das Nachdenken darüber ist mein Anliegen hier.

So zählt, was nun als zweiter Hälfte dieses Buches folgt, als Gegenfrage zum Diskurs um die Digital Humanities. Und ist eben keine Position gegen den Wert der Digital Humanities. Ganz im Gegenteil, wie Ted Underwood in einer Verteidigung digitaler Literaturwissenschaft schon vor einem guten Jahrzehnt erklärt hat, als er auf jene anstehenden Konflikte um die Digital Humanities vorausschaute, in denen wir uns tatsächlich jetzt am Ende des Jahrzehnts befinden:

»Over the next several years, I predict that we're going to hear a lot of arguments about what the digital humanities can't do. They can't help us distinguish insightful and innovative works from merely typical productions of the press. They can't help us make aesthetic judgments. They can't help students develop a sense of what really matters about their individual lives, or about history. [...] The best way to get people to pay attention to the humanities is for us to have a big, lively argument about things that matter - indeed, I would go so far as to say that no humanistic project matters much until it gets attacked. And critics of the digital humanities will be pointing to things that really do matter. We ought 
to be evaluating authors and works, and challenging students to make similar kinds of judgments. We ought to be insisting that students connect the humanities to their own lives, and develop a broader feeling for the comic and tragic dimensions of human history. Of course, it's not as though we're doing much of that now. But if humanists' resistance to the digitization of our profession causes us to take old bromides about the humanities more seriously, and give them real weight in the way we evaluate our work - then I'm all for it. (135 $^{13}$

Am Ende gehört das Folgende aber über die Frage nach den Möglichkeiten und Grenzen der Digital Humanities hinaus zugleich auch zu dem, was Lawrence Kramer umtreibt, wenn er schreibt: »The topic is the formation of auditory experience in and through the accounts we give of it. $"{ }^{136} \mathrm{Im}$ Anschluss an Latours >matters of concern gründung im Schlussteil dieses Buches daher einen ausgewählten Aspekt meines eigenen ästhetischen Erlebens des Klavierquintetts in den Mittelpunkt rücken. Nämlich das, was Paul Guyer in seiner History of Modern Aesthetics »emotional impact« genannt hat. ${ }^{137}$ Mein >matter of concern hier. Eine der auf Rezeptionsseite erkundbaren ästhetischen Qualitäten der Sache. Und diese ins Verhältnis setzen zur besagten Qualitätsunterstellung der skizzierten Kernrepertoirebildung, die das Genre so sehr prägt. Dabei wird es darum gehen, zu prüfen, ob ich beschreiben kann, wo genau der »emotional impact « in dem zu verorten ist, was ich höre. Und ob ich dies so beschreiben kann, dass zumindest ich selbst mein eigenes ästhetisches Erleben in den Beschreibungen wiedererkenne. ${ }^{138}$ Oder um dieses Ziel nochmals mit Latour zu bündeln: »My question is thus: Can we devise another powerful descriptive tool that deals this time with matters of concern $\ll ?^{139}$ Um im Anschluss in Ludwig Wittgensteins Sinne den Bezugspunkt dieses »emotional impact« in der jeweiligen Musik zeigen zu können. ${ }^{140}$ »Betrachten« zu ermöglichen, wie Wittgenstein sagt. ${ }^{141}$ Nicht bloß um seiner selbst willen. Sondern um dieses Zeigen dann als eine alternative Zugangsweise zu Musikgeschichte nicht nur zu erkunden, sondern als legitime und produktive, nicht bessere, aber für mich am Ende eben insbesondere notwendige parallele Zugangsweise zu reklamieren und zu verteidigen. In einer Argumentationsline von Joseph Kerman und Albrecht Riethmüller bis Lawrence Kramer und Nicholas Cook als Perspektive auf Musikgeschichte stark zu machen. ${ }^{142}$ Nicht obwohl, sondern gerade weil mein primäres Aufgabenfeld derzeit - und dies mit großem Interesse - die 
Digital Humanities sind. ${ }^{143}$ Und damit eine dem hiesigen Anliegen genau entgegengesetzte Annäherungsweise an Musik.

Die Spezifik der Soziologie des Klavierquintettgenres prädestiniert es als Fallbeispiel für dieses Unternehmen hier. Denn das Genre des Klavierquintetts erlaubt es aufgrund seiner Spezifik schlicht nicht, wie andere Gattungen Klassischer Musik als Einflussgeschichte erzählt zu werden. Oder als Heroenmusikgeschichte. Es wäre nicht viel zu holen. Man kann hier aber auch nicht einfach auf selbstevident tun. Markennamen lassen sich nicht vorschieben. Wie es so typisch ist für Musikgeschichtskonstruktion und -tradierung. Gerade für solche, die das eigene ästhetische Erleben als historiographisch relevante Kategorie zurückweist. In dem hier regelmäßig die eigenen Werturteile als tragendes Moment für Auswahlentscheidungen verschwiegen werden, wie Frank Hentschel herausgearbeitet hat:

»Musikgeschichtsschreibung, so lautet die kurz gefasste These der Arbeit, stützt sich immer auf ästhetische Urteile, deren Begründung jedoch nach Johann Nikolaus Forkel von den Musikhistorikern nicht mehr ausdrücklich vorgenommen wurde. $\aleph^{144}$

Im Fall des Klavierquintetts lässt sich die Autoritätslücke für eigene Auswahlentscheidungen aber eben nicht durch Autoritätstransfer von Markennamen schließen, wie Nicholas Cook und Mark Everist am Beispiel des Theoriediskurses als übliches Vorgehen beschrieben haben:

»Not surprisingly, then, many musicologists have sought to resolve - or at least to evade - such uneasy tensions through an unquestioning adherence at all costs to some fixed theoretical point; not just Schenker or Marx, but Adorno, Benjamin, and even Dahlhaus have acquired the status of authorities who do not require $[\ldots]$ question or challenge. [...] And it is the notion of authority that is cen$\operatorname{tral}[\ldots] . \ll^{145}$

Man muss beim Klavierquintett also nach alternativen Zugangsweisen suchen und für diese streiten. Das Problem ist also nicht das Auswählen. So wie es die Leitmedien mit ihrem Kernrepertoire tun. Es muss sein. Die Tradierung von Musikgeschichte kann aufgrund der Überfülle von Musikern und Musiken, der dazugehörigen Ressourcen samt dem diesbezüglichen Fortschreiten der Forschungsarbeit und schließlich dem Umstand, 
dass ständig neue Musikgeschichte geschaffen wird, die nach Aufmerksamkeit ruft, gar nicht anders als auswählend vorgehen. Historiographisch muss Auswählen sein, um sich nicht in Datenbanken, Chroniken und Statistiken zu verlieren. Aber ohne die Möglichkeit des Autoritätstransfers über Markennamen ist man plötzlich angehalten, aktiv zu rechtfertigen, was man warum auswählt. Dafür zu streiten und zu werben. Gerade auch als $\mathrm{Mu}-$ sikhistoriker. Darüber nachzudenken, wovon man eigentlich erzählen will. Die Eigenart des Klavierquintettgenres zwingt einen dazu. Das hat etwas ungemein Befreiendes. 



\section{Ästhetik des Klavierquintetts: Situatives Hören, Emotional Impact und das Kuratieren alternativer Musikgeschichte}

\section{Situatives Hören als Perspektive}

Der zweite Teil dieses Buches schließt an den ersten an, hat aber ein völlig anderes Anliegen. Die verschiedenen Kapitel markieren diametral entgegengesetzte Annäherungsweisen an denselben Gegenstand. In Ästhetik des Klavierquintetts geht es um das Genre als spezifische musikalische Praxis. Um die Kombination von Klavier und Streichquartett als charakteristisches Soundscape. Um individuelle künstlerische Lösungen, die innerhalb dieses vorgegebenen Settings operieren. Ganz konkret in Gestalt einer Auswahl von Werken als Tonaufnahmen, ja einzelner musikalischer Passagen, Episoden, Momente in diesen Realisierungen.

Nachdem ich eben also ein hunderte Werke starkes, über zwei Jahrhunderte altes Genre in gewisser Weise als kohärente Entität betrachtet habe, interessiert im Folgenden das genaue Gegenteil: ausgewählte musikalische Situationen in einzelnen Klavierquintetten, oft nur wenige Sekunden und Takte lang. Einzelbeobachtungen also. Situatives Hören als Perspektive auf den Gegenstand.

Als wenn ich bei Vincent Van Goghs Gemälde Die Brücke von Langlois bei Arles (1888, Fassung mit Dame und Sonnenschirm) nur über die filigrane Struktur und Linienführung der Seile der hölzernen Zugbrücke spräche. ${ }^{146}$ 
Oder bei Elia Kasans Verfilmung (1951) von Tennessee Williams' Theaterstück $A$ Streetcar Named Desire (1947) nur über die Intensität von Vivien Leighs alias Blanche DuBois' Gesichtsausdruck, wenn sie vor Karl Malden alias Mitch kniet und ihm im Streit entgegenschleudert: »I don't want realism! I want magic! « ${ }^{147}$

Oder bei der Realisierung der Choreographie von Kenneth MacMillan für Covent Garden (1965, Filmfassung von 1966) von Sergej Prokofjews Ballett Romeo und Julia op. 64 (1938) auf William Shakespeares Theaterstück (1597) durch Margot Fonteyn alias Julia und Rudolf Nurejew alias Romeo nur über den Dialog der Gesten zwischen den beiden, wenn sie sich in der Balkonszene das erste Mal berühren, nachdem Julia die Treppe heruntergeeilt ist, Romeo nicht sofort sieht und er von hinten an sie herantritt. $^{148}$

Oder nur über den Augenblick in F. Scott Fitzgeralds The Great Gatsby (1925), wenn in der Plaza-Szene Daisy Buchanan Gatsby entgegenruft:

") Oh, you want too much!< she cried to Gatsby. >I love you now - isn't that enough? I can't help what's past.< She began to sob helplessly. \I did love him once - but I loved you too. « ${ }^{149}$

- und er schlagartig alles verloren hat und seine sorgsam etablierte Position der Stärke für alle sichtbar mit einem Wimperschlag in sich zusammenfällt, nur für ihn selbst noch nicht.

Mein Anliegen hier ist also gerade keine Analyse, die vollständige Klavierquintette in den Blick nimmt. Oder gar deren Gesamtinterpretation. Im Gegenteil. Auch Vergleiche verschiedener Darbietungen erfolgen nur punktuell. Erst recht schreibe ich keine umfassende Kompositionsgeschichte des Genres, etwa durch Gegenüberstellung von verschiedenen Klavierquintetten. So wie es für diese musikalische Praxis am ehesten noch Basil Smallman 1994 unternommen hat. ${ }^{150} \mathrm{Ob}$ alle >wichtigen` Werke und Komponistennamen vorkommen, was auch immer sie in Historiographie, Aufführungspraxis und Musikwirtschaft dazu macht, spielt dementsprechend keine Rolle. Anders als im ersten Kapitel. Und es wird tatsächlich am Ende auch nicht hiernach ausgewählt worden sein. Eine Anleitung und Orientierungshilfe im Repertoire für Aufführende mit Darbietungshinweisen zu Schwierigkeitsgrad etc., wie sie z.B. Wilhelm Altmanns klassisches Handbuch zum Klavierquintett von 1936 anbietet, ist dies ebenfalls nicht. ${ }^{151}$ 
Wenn Carl Dahlhaus die Konzentration auf das Schreiben eines historiographischen Metanarrativs - für mich stets Metahistory im Sinne Hayden Whites ${ }^{152}$ - mit den Worten beschreibt und begründet,

»Die Frage, was ein Werk für sich, als isoliertes, in sich geschlossenes Gebilde sei und bedeute, wird abgelöst von dem Versuch, die Stellung zu bestimmen, die es im geschichtlichen Prozess einnimmt «, ${ }^{153}$

so ist es genau das, wovon er diesen übergeordneten - oder besser: überordnenden - Zugriff auf Musikgeschichte abgrenzt, was mich hier interessiert. Klavierquintette als isolierte, in sich geschlossene Gebilde. Genauer noch: einzelne Situationen in eben diesen Gebilden.

\section{Herleitung der Perspektive}

Warum diese diametral entgegengesetzte, fast schon mikroskopisch konzentrierte Annäherungsweise an denselben Gegenstand?

Der erste Teil dieses Buches war eine musiksoziologische Annäherung an die Mechanismen hinter einer - so die Diagnose - markennamengetriebenen Musikgeschichtstradierung. Innerhalb derer der Fokus gerade nicht, wie im Musikleben weithin suggeriert, auf den (wie auch immer ermittelt) >besonders guten`Werken liegt, sondern auf einem vergleichsweise kleinen Kanon von Genres und vor allem Komponistennamen, die wiederum diese Genres dominieren. Genres wie Oper, Sinfonie oder Streichquartett. Man trifft auf Mechanismen, die im Gegenteil mit verblüffendem Desinteresse gegenüber der Qualitätsfrage jenen des Managements von Produktmarken auffallend nahe kommen. Bis hin zu einer Verehrung irgendwo zwischen quasi-religiöser Überhöhung und Fetisch. Das Produkt mag exzellent sein oder nicht. Was im Zweifel zählt ist aber der Wert der Marke. Sie ist die eigentliche Basis des Kommunikationshandelns. Sie gilt es zu reklamieren und $\mathrm{zu}$ verteidigen.

Getragen war der erste Teil dabei von der Diagnose, dass diese generell greifenden Mechanismen aufgrund der Geschichte des Klavierquintetts anhand Spezifik dieses Genres hier in besonders pointierter Weise sichtbar werden. Der Soziologie des Klavierquintetts war es also um verallgemeinerbare Aussagen über typische, im herrschenden Diskurs dominante Um- 
gangsweisen mit Klassischer Musik und ihre Folgen gegangen. Kontrastiert mit dem Versuch, auf aktuelle Veränderungen hinzuweisen, welche die Macht dieser Mechanismen herausfordern. Entsprechend ging es im ersten Teil um eine Makroperspektive auf das Klavierquintett. Auf das einzelne Werk kam es hier nicht an, um besagte Diagnose zu stützen, ebenso wenig wie auf eine bestimmte Aufführung oder Aufnahme. Letztlich muss die Diagnose im Gegenteil, sollte sie denn wie von mir behauptet belastbar sein, sogar implizieren, dass es am Ende nicht einmal auf das Klavierquintett als solches ankommt, d.h. auf seine ästhetische Spezifik als Genre insgesamt. Dieses hat an der dortigen Stelle viel mehr exemplifikativen Charakter.

Das Klavierquintett ist für mich aber mehr als abstrakte Gattung, oft genutztes Format, langlebiges Genre - und als Datensammlung wissenschaftliches Mittel zum argumentativen Zweck. Für die Beweisführung des ersten Kapitels bleibt das Klavierquintett zwangsweise in seinen einzelnen Beiträgen austauschbar. Reduziert auf die schiere Quantität und Kontinuität seiner Gesamtproduktion, die Namen seiner Protagonisten, seine Präsenz im Konzertleben und unter den Notenausgaben und Tonaufnahmen Klassischer Musik. Aber das ist eben nicht alles. Ich höre einen Großteil dieser Musik tatsächlich. Seit langem. Immer noch. Und als Hörer ist das Genre für mich in seinen einzelnen Beiträgen gerade nicht austauschbar. Das Gegenteil ist der Fall. Ich habe hier »matters of concern«, wie Latour sagt. ${ }^{154}$

Der so anders geartete Blickwinkel des zweiten Teils nimmt von dieser Selbstbeobachtung seinen Ausgang. Deswegen ist das Folgende derart zugespitzt und konzentriert auf bestimmte musikalische Situationen in einzelnen Klavierquintetten. Suchte das eröffnende Kapitel etwas zu beschreiben, das mir ganz generell das Metier der Klassischen Musik zu prägen scheint, suche ich im Folgenden musikalische Besonderheiten für mich zu verstehen und soweit mir möglich für andere zu beschreiben. Und zwar eben nicht das Besondere im Verhältnis zum Allgemeinen, so Martin Seel, wie es die übliche Aufgabe der Ästhetik ist. ${ }^{155}$ Nicht das absolut Besondere, objektiv Besondere also, das kompositionsgeschichtlich Besondere etwa - sondern schlicht das für mich Besondere. Die musikalischen Situationen, an denen ich stets hängen bleibe, die meine Aufmerksamkeit binden, mich als Hörer emotional involvieren. ${ }^{156}$ Mich beschäftigt also, wie Albrecht Wellmer schreibt, 
»die Frage, was die Musik nicht nur für den komponierenden und aufführenden Musiker, sondern auch für die Musikhörer bedeutsam und wichtig macht « ${ }^{157}$.

In meinem Fall mehr noch: nicht auch bedeutsam macht für Musikhörer, sondern gerade. Und nicht für irgendwelche Musikhörer. Oder den $\mathrm{Mu}-$ sikhörer an sich als objektivierte, normalisierte Größe. Sondern für mich als Musikhörer.

»Ziel dieses Erkennens ist nicht das - durch Klassifikation und Generalisierung zu erfassende - Allgemeine, sondern die Beachtung des Besonderen. Das Besondere in seiner Besonderheit zu erkennen, $[\ldots] \ll^{158}$

wie Seel seine Lektüre von Alexander Gottlieb Baumgarten zusammenfasst - darum geht es mir.

\section{„Emotional Impact« Als Fokus der Perspektive}

Der zweite Teil dieses Buches ist damit unverkennbar »academic music criticism « im Geiste Joseph Kermans. ${ }^{159}$ Akademisch zwar, da stets wissenschaftlich gemeint in Richard Taruskins Sinne einer »proper doubting, interrogatory, and consciousness-raising role« dessen, was hier sogleich versucht werden wird. ${ }^{160}$ Aber doch etwas anderes als z.B. das erste Kapitel. Nämlich nun als ausdrücklich wertorientierte, da wertende Musikkritik gemeint. Ich sage, was ich gut finde und warum. Offen. Ohne Netz und doppelten Boden. Ganz so, wie Noël Carroll es in On Criticism als Aufgabe des Kritikers beschrieben hat:

»For me, the primary function of the critic is not to eviscerate artworks. Rather, I hypothesize that the audience typically looks to critics for assistance in discovering the value to be had from the works under review. «161

Dabei ist mein Unternehmen in seiner Suche nach ästhetischem Wert versehen mit einem klaren Fokus auf die Relevanz der Sache für das Hier und Jetzt. Für mich in meiner Gegenwart. Darin folge ich Elijah Walds zentralem Charakteristikum für »academic music criticism«: 
»The critic's job is to assign value and importance on an artistic level, which necessarily is a judgement about how the work stands up in the present. ${ }^{162}$

D.h., es geht hier gerade nicht darum, die Vergangenheit in ihrer Zeit verstehen zu wollen - ein oft faszinierendes Unterfangen, aber eben doch ein dezidiert ganz anders geartetes. Sondern die Gegenwart des Klavierquintetts. In dieser Hinsicht also ganz so wie im ersten Kapitel auch.

Ausgehend von diesen beiden Prämissen Carrolls und Walds, ist es dann ein ganz spezifischer ästhetischer Wert für das Hier und Jetzt, der von mir mit Paul Guyer in den Blick genommen wird: »emotional impact $\ll .{ }^{163}$ Guyer verwendet »emotional impact« als einen zentralen Begriff in seiner monumentalen History of Modern Aesthetics. Für Guyer ist »emotional impact« eine der wesentlichen Neuerungen der Ästhetik des 18. Jahrhunderts (die beginnend mit Alexander Gottlieb Baumgartens unvollendet publizierter Schrift Aesthetica von 1750/58 überhaupt erst diesen Terminus ausprägt). Eine Neuerung, die sich zu einer »distinct line of thought« in der Ästhetik verdichtet - freilich mit einer problematischen Geschichte, wie Guyer ausführlich beschreibt. ${ }^{164}$

Aus meiner Sicht ist »emotional impact« eine sehr glückliche Formulierung. Denn sie bezeichnet als Terminus in sich schon sehr genau, worum es geht. ${ }^{165}$ Klarer etwa, als wenn man, wie klassischer Weise üblich, von "schönen Stellen« spricht. D.h. von musikalischen Momenten, die - neutral gesprochen - die eigene ästhetische Aufmerksamkeit in herausgehobener Weise binden, wie Theodor W. Adorno erläutert. ${ }^{166}$ Darum geht es mir zwar auch. Aber eben nur in einer bestimmten Hinsicht, mit einem spezifischen Fokus. »Emotional impact« macht unmissverständlich klar, dass die Rezeptionsperspektive gemeint ist. Momente intellektueller Faszination an den Gegenständen, an ihrer Innovationskraft oder ihrer technischen Meisterschaft oder ihrem historischen Einfluss, sind hier also nicht das Thema. Auch nicht, wie jener Ausdruck impliziert, »Schönheit« als Qualität der »schönen Stellen« selbst. Nicht nur haben die Moderne wie die Postmoderne dies aus so vielfältigen Gründen und in so interessanten Weisen als künstlerisch relevante Kategorie diskreditiert, wie Arthur C. Danto zeigt. ${ }^{167}$ »Schönheit« als beschreibbarer Qualität der Gegenstände selbst nachzuspüren, erscheint mir mit David Hume überhaupt einigermaßen müßig: 
"All sentiment is right; because sentiment has a reference to nothing beyond itself, and is always real, wherever a man is conscious of it. [...], a thousand different sentiments, exited by the same object, are all right: Because no sentiment represents what is really in the object. It only marks a certain conformity or relation between the object and the organs or faculties of the mind; [...]. Beauty is no quality in things themselves: It exists merely in the mind which contemplates them; and each mind perceives a different beauty. ${ }^{168}$

Aber nicht nur geht es nicht um die Schönheit »schöner Stellen«. Es geht in keiner Hinsicht um die emotionale Expressivität einer bestimmten Musik. Einer Expressivität, die ihr eingeschrieben ist, ob ich sie selbst nun so nachempfinde oder nicht. ${ }^{169}$ »Emotional impact« als Begriff macht klar, dass die Rezeptionsperspektive im Vordergrund steht. Er betont ferner, dass dies ausschließlich auf der Ebene der sinnlichen Wahrnehmung geschieht. Anliegen ist nicht, Emotion oder Schönheit oder was auch immer im Gegenstand selbst dingfest zu machen. Das ästhetische Erleben ist hier das Thema. Von spezifischen musikalischen Situationen, die etwas sind, das Hans Ulrich Gumbrecht treffend »Augenblicke der Intensität« nennt. ${ }^{170}$ Und Ben Ratliff als »perfect moment« näher umschreibt:

»A perfect moment is often wordless, or indirect if it has words. It is the song blushing: an unplanned or perhaps only semiplanned occurrence in which the music suddenly embodies its own meaning. The conscious mind of the singer or the instrumentalist temporarily goes out the window. It can contain everything of value about the piece of music in one note or chord, or a very short sequence of time. It communicates a complicated human gesture, feeling, or interaction that could not be literally demonstrated or sung; it transcends or surpasses the listener's expectation set up by context in order to create a moment of beneficial confusion or satisfaction. It is the song turning itself inside out for you, sleepwalking or convulsing or speaking a temporary truth. ${ }^{171}$

Letztlich geht es mir also bei »emotional impact«, mit Heinz Schlaffer gesprochen, um die »Wirkung schöner Stellen, [...] deren intensive Präsenz und die oft lebenslang anhaltende Zuneigung zu ihnen «. ${ }^{172}$ Man könnte mit Gumbrecht traditioneller auch von Epiphanien sprechen. ${ }^{173}$ Nur, dass diese in besagten musikalischen Situationen für mich in ihrem »emotional impact« gezielt wiederholbar, ja durch wiederholtes und hierdurch vertiefend 
informiertes Hören in der spezifischen Erfahrung, die sie mir ermöglichen, intensivierbar sind.

Die Qualifizierung lebenslang ist im Übrigen wichtig. Sie zeigt eine Nachhaltigkeit an. Alle später betrachteten musikalischen Situationen haben sich in der Tat in ihrem »emotional impact« als ausgesprochen nachhaltig und belastbar erwiesen. Ich habe nur solche aufgenommen. Es gibt andere, die nur eine temporäre Wirkung entfaltet haben. Und auf die man mit Abstand zurückschaut und nicht mehr recht weiß, warum man sie einmal geliebt hat. Das hierarchisiert die Fälle nicht. Das eine ist nicht wichtiger oder betrachtenswerter oder beschreibungswürdiger als das andere. Aber es ist eben etwas anderes. Und es ist das Erste, worauf ich mich hier konzentriere. Der Frage nachzugehen, warum einem manches bleibt und anderes nicht, wäre ein Thema für sich. Nicht weniger spannend, weil man wahrscheinlich nicht weniger über sich und seinen Verhältnis zur Musik dabei lernt. Aber doch vorbehalten für einen anderen Tag.

Zwar spreche ich damit in meinen nachfolgenden Exkursen in und über diverse musikalische Situationen in Klavierquintetten, wie Stefan Majetschak präzisiert, zuvorderst

»gar nicht über eine Eigenschaft des Gegenstandes, sondern bloß über eine im Zuge der Reflexion über dessen Wahrnehmung sich einstellende Befindlichkeit des urteilenden Subjekts selbst $\ll .{ }^{174}$

Aber es hat eben doch zugleich wieder mit diesen musikalischen Reflexionsgegenständen selbst zu tun, wie Martin Seel hervorhebt, denn

»Kunstwerke sind Objekte, die in einer bestimmten Weise aufgefaßt werden. Das aber, was in diesen unterschiedlichen Arten der Wahrnehmung zur Erfahrung kommt, ist keine Projektion; es ist nichts, was nicht wirklich da wäre. «175

Guyer beschreibt entlang seines Begriffs vom »emotional impact«, wie eben dieser im ausgehenden 18. Jahrhundert in der ästhetischen Theorie beginnt, mehr und mehr außen vor zu bleiben. Spätestens ab Kant hat die Emotion in der Ästhetik einen schweren Stand in der Begründung der Spezifik und Relevanz ästhetischer Erfahrung wie des Wertes von Kunst. ${ }^{176}$ (Und in der Musikwissenschaft seit Eduard Hanslick, wie Nicholas Cook ergänzt. ${ }^{177}$ ) Abgeschoben in die vermeintlichen Untiefen von Journalismus 
und Alltagsgespräch. Und verpönt als wissenschaftliche, an Erkenntnis interessierte Zugangsweise. Mit Joseph Kerman halte ich dies für einen Fehler, eben weil »emotional impact«, mit Seel gesprochen, mit der Sache selbst zu tun hat und keineswegs von außen an sie herangetragen, lediglich auf sie projiziert ist:

»But with the majority of them [musicologists], in my experience, it is not so much a matter of inherent unmusicality as of a deliberate policy of separating off their musical insights and passions from their scholarly work. I believe this is a great mistake; musicologists should exert themselves towards fusion, not separation. When the study of music history loses touch with the aesthetic core of music, which is the subject matter of criticism, it can only too easily degenerate into a shallow exercise. ${ }^{178}$

Die Folgen sind weitreichend, wie Susan McClary ergänzt:

»Consequently, >the music itself^ continues in most quarters to maintain its status as ineffable, immune from the kinds of debates that take place as a matter of course within literary, film, ort art-historical studies. « ${ }^{179}$

Sich auf »emotional impact« als Agenda einzulassen, ist daher für mich als Zugangsweise wesentlich. Aber es ist keine Selbstverständlichkeit. Dass stattdessen meist sogar »Ästhetiker dafür optieren, die Werke selbst unabhängig davon $\mathrm{zu}$ untersuchen, wie sie erlebt werden «, ${ }^{180}$ hat vielfältige Gründe, einigen Ertrag und eine lange Tradition, wie Albrecht Riethmüller ausführlich beschrieben hat, ebenso wie die damit zusammenhängende

»Melange von sich überschneidenden und gegenseitig überlagernden, sich stützenden und zugleich ineinander opponierenden Gedanken - einerseits eine Art Objektivitätsideal des Komponierens und Musizierens, andererseits einer Reserviertheit gegenüber Gefühl und subjektivem Ausdruck «. ${ }^{181}$

Freilich handelt es sich dabei oftmals um eine lediglich vorgeschobene Position, wie Frank Hentschel ergänzt. ${ }^{182}$ Die unglaubwürdig ist. Denn wer »emotional impact« nicht fühlen, »Augenblicke der Intensität« nicht zulassen und »perfect moments« nicht erkennen kann, sei schlicht »taub«, wie Adorno in seiner Ästhetischen Theorie anmerkt. ${ }^{183}$ Und das dürfte den 
wenigsten zu unterstellen sein. Vielmehr handelt es sich um eine strategische Entscheidung, wie Kerman anmerkt. D.h., dass es sich in den meisten Fällen um mit Absicht gesetzte Leerstellen im Sinne der vierten von Michel-Rolph Trouillots »silences« handelt,

»silences enter the process of historical production at four crucial moments: the moment of fact creation (the making of sources); the moment of fact assembly (the making of archives); the moment of fact retrieval (the making of narratives); and the moment of retrospective significance (the making of history) «. ${ }^{184}$

Bevorzugt ersetzt schließlich von jener »Vorstellung von musikalischen Kunstwerken, deren Wert gerade nicht verhandelbar, sondern intrinsisch ist ${ }^{185}$, wie Friedrich Geiger und Tobias Janz schreiben.

Mir jedoch scheint dies nicht nur in vielen Fällen unaufrichtig und vorgeschoben zu sein, sondern vor allem auch unproduktiv. Eine solche Position verpasst nämlich notwendig wesentliche Facetten des Ganzen, wie Kerman und Seel anmahnen. Schon Baumgarten hat hierauf in seiner Aesthetica hingewiesen, wenn er reklamiert, dass die sinnliche Wahrnehmung auch erkenntnisfördernd ist. ${ }^{186} »[\mathrm{~A}]$ uf die Wahrnehmung komplexer Phänomene spezialisiert«, letztlich gar zwingende Voraussetzung ist für ein »>vollständiges` Erkennen«, das »nur durch wissenschaftliches und ästhetisches Denken erreicht werden « kann, wie Seel zuspitzt. ${ }^{187}$

Ich jedenfalls will Offenheit. Will verstehen, warum jemand für etwas streitet. Wo seine Faszination liegt. Vielleicht überzeugt es mich ja? Zeigt mir einen Zugang, den ich so noch nicht gesehen habe? Und da ist wenigstens für mich »emotional impact« ein genauso legitimes, valides und gleichwertiges Argument, etwas zur näheren, gar wiederholten Beschäftigung auswählen, wie das Maß an Kunstfertigkeit oder der Innovationsgrad, der Einfluss als Vorbild auf die Geschichte eines Genres oder die soziale Resonanz einer Arbeit, um nur einige der anderen denkbaren Blickwinkel zu nennen. Oder wie im ersten Kapitel angedeutet, als strategisches Mittel der Beweisführung zur Dekonstruktion von letztlich unaufrichtig auftretenden sozialen Umgangsweisen mit Musik.

Etwas als besonders auf andere Weise als durch Abgleich mit dem Allgemeinen >zeigen zu wollen`, ist natürlich riskant, wie Ratliff warnt: 
»Perhaps here we're on the thinnest ice of subjectivity. Is my perfect moment your perfect moment? Possibly. No guarantees there. $\ll^{188}$

Man kann nicht sagen: 〉Es ist.২ Sondern nur: >Ich finde.২ Und versuchen, dies nachvollziehbar, im besten Fall vielleicht durch die Qualität der Beschreibung als Erfahrung sogar nacherlebbar zu machen. Man muss nicht gleich auf eine Ekphrasis hoffen. Aber in der Verbindung von Beschreibung und Nachhören scheint doch erreichbar, die eigene Hörperspektive für jemand anderen einnehmbar zu machen.

Das macht natürlich nur Sinn, wenn man von der Kantschen Idee der Teilbarkeit der dahinter stehenden ästhetischen Erfahrungen und Urteile ausgeht. ${ }^{189}$ Und auch das ist allemal eine heikle Angelegenheit, worin ich A. O. Scott uneingeschränkt beipflichte, wenn er einwendet:

") Subjective universality< - the notion that what is beautiful to one is beautiful to all [...]. Our age does not feel comfortable with the idea of subjective universality. The phrase sounds a bit too presumptuous, too coercive, for our sensitive, pluralistic postmodern ears. $\ll^{190}$

Unter anderem deswegen beanspruchen meine nachfolgenden Situationsbeschreibungen für sich auch nicht, mehr zu sein als das, was Scott in Form einer Gegenfrage formulierte:

»[Criticism] has always been part of the landscape, though, arising from our desire - nearly as strong as the urge toward pleasure itself - to think about, recapture, and communicate our delights, to make them less solitary, less ephemeral. The origin of criticism lies in an innocent, heartfelt kind of question, one that is far from simple and that carries enormous risk: Did you feel that? «191

Genau diese Frage zu stellen, ist mir jedoch wichtig. Eine ganze Reihe Gründe kommen dabei zusammen, warum ich ihr hier einmal nachgehe. Warum ich an dieser Stelle mit Absicht anders verfahre. Diametral anders als im ersten Kapitel für mein laufendes Pilotprojekt an der Deutschen Nationalbibliothek skizziert. Anders aber auch als im Gros meiner sonstigen Forschungsarbeit. Insbesondere die Übernahme jener Verantwortung für den Bereich der Digital Humanities im Leitungsstab der Generaldirektion der Deutschen Nationalbibliothek hat zuletzt meinen Blick für die 
konkrete ästhetische Einzelheit, die Spezifik des künstlerisch-expressiven Details, und ihre ganz persönliche Wahrnehmung paradoxerweise enorm geschärft und mein reflexives Interesse hieran potenziert. Denn sich mit Digital Humanities zu beschäftigen, heißt eben, sich mit computerunterstützter Forschung auf Basis großer Datenmengen auseinanderzusetzen. ${ }^{192}$ Oft faszinierender Forschung. Auf der Suche nach Mustern, Trends und Korrelationen verschwindet aber rasch gerade die konkrete ästhetische Einzelheit, wird ihre Spezifik wie ihr ästhetisches Erleben zum statistisch Vernachlässigbaren. Noch weit über jene Verallgemeinerung des Klavierquintettgenres hinaus, die hier aus musiksoziologischer Perspektive schon das erste Kapitel trug. Auch deswegen wird im hiesigen Abschnitt im Vergleich zum vorangegangenen der Blickwinkel auf den Gegenstand von der Makro- zur Mikroperspektive radikal verschoben. Aufgrund der Provokation der Digital Humanities, die Berechtigung zu hinterfragen, sich überhaupt noch als Wissenschaftler für die konkrete ästhetische Einzelheit zu interessieren. ${ }^{193}$ Dies zu tun zu marginalisieren mit dem Gegenpostulat eines »metrischen Paradigmas «. ${ }^{194}$ Manche Forschungsperspektiven scheinen mir aber unbeeindruckt von Big Data jedweder Art »subjectivity, aesthetics, interpretation, and emergent phenomena « zu fordern und »qualitative, interpretive, experiential, emotive, and generative in nature « zu sein, wie Julie Thompson Klein im Rekurs auf Johanna Drucker reklamiert. ${ }^{195}$ Das, worum es mir in diesem Kapitel geht, gehört für mich genau hierzu.

In Zeiten, in denen mit Nachdruck institutionell und finanziell geförderte disziplinäre Zugriffe auf Musik von Digital Humanities bis Neuroästhetik eine Vernaturwissenschaftlichung der Auseinandersetzung betreiben, spüre ich, dass mir etwas fehlt. Dass ich in Baumgartens Geiste einen anderen Zugang als Korrektiv und Ergänzung des Bildes mir neben das halten möchte, was auf anderem methodisch-theoretischem Weg an Verallgemeinerung und - meist wertvollem, oft gar faszinierendem - Allgemeinen gewonnen wird. Ich bin also an einem ganz ähnlichen Punkt wie dem, den Bruno Nettl beschrieben hat, als er von einem Rat des Anthropologen Bronisław Malinowski an ihn berichtet:

»But Malinowski warned: Do not just accumulate data but constantly interpret. Thus, instead of amassing a lot of data, which should certainly be done, I decided that I might best contribute something by taking an interpretive perspective. ${ }^{196}$ 
Die in meinem Fall von mir an dieser Stelle gewählte, dem allgemeinen Trend entgegengesetzte interpretative Perspektive folgt nun der Erfahrung, dass ich oft besonders großen Gewinn davontrage, wenn mir jemand konkret zeigt, was sie oder ihn ganz persönlich erreicht. D.h.: »emotional impact« entfaltet. Meinen Blick genau darauf lenkt. Sicht traut, öffnet und mir im besten Fall einen Nachvollzug ermöglicht. Und so vielleicht meine »Anschauungsweise« ändert dahingehend, »einen gegebenen Fall anders zu betrachten«, wie es bei Ludwig Wittgenstein heißt. ${ }^{197}$

Das sagt sich leicht, ist aber eine große Sache. Und entsprechend selten. Umso seltener, je gebildeter und qualifizierter der Umgang von Autoren oder Gesprächspartnern mit Musik ist. Viele mögen es nicht, diese Perspektive zuzulassen. Schließlich macht man sich auf gewisse Weise angreifbar. ${ }^{198}$ Festzustellen nämlich, dass das, was einen berührt, jemand anderen kalt lässt, ist eine einigermaßen frustrierende Erfahrung. Als ob es die eigene Emotion in Frage stellen würde. Dagegen z.B. über eine formale Frage uneins zu sein, ist ein intellektuelles Problem. Uneinigkeit scheint hier leichter zu ertragen zu sein. Hinzukommt, wie Elijah Wald anmerkt, die Tendenz des »academic music criticism«, musikalische Phänomene und Musik insgesamt höher zu schätzen und stärker zu beachten, je mehr sie etablierten, ausgefeilten Analyseinstrumenten zugänglich ist und eine vertiefte Annäherung auf diesem Weg gesichert scheint. ${ }^{199}$ »Emotional impact« tut sich hier schwer. Aber es muss nicht so bleiben: »One person’s trying it may encourage others to do the same in their own words«, wie Lawrence Kramer anmerkt. ${ }^{200}$

»Emotional impact« ästhetischer Erfahrung ist jedenfalls genau das, was ich zumindest in Musik am liebsten finde und am häufigsten suche. Andere wenden sich hierfür vielleicht eher einem Buch oder einem Theaterstück zu. Einer Landschaft oder einem Fußballspiel. Mitmenschen oder Religion. Wechselnd allem zusammen. Oder zwar Musik, aber vielleicht ganz anderer als jener, die mich erreicht. Und »emotional impact « ist beileibe auch nicht das einzige, was mich an Musik interessiert, wie das erste Kapitel illustriert. Dafür bin ich zu sehr Wissenschaftler. Aber dass »emotional impact« in der akademischen Rezeption des Klavierquintetts argumentativ als Wert eine derart untergeordnete Rolle gegenüber z.B. dem Wert von Markennamen spielt, wie mir evident zu sein scheint und wie ich zu beschreiben versucht habe, hat mich bei der Arbeit am ersten Kapitel in 
ganz grundlegender Weise provoziert. ${ }^{201}$ Auch deswegen gibt es diesen zweiten Teil. Und wurde der erste nicht ein solitär bleibender Aufsatz.

Letztlich bin ich mit der hier erkundeten Perspektive an einer Stelle angelangt, die Susan Sontag schon vor einem halben Jahrhundert beschrieben hat. In ihrem Essay Against Interpretation in der Argumentation, die der berühmten, viel zitierten Schlusszeile »In place of a hermeneutics we need an erotics of art« direkt vorausgeht und argumentativ zu ihr hinführt:

»Interpretation takes the sensory experience of the work of art for granted, and proceeds from there. This cannot be taken for granted, now. Think of the sheer multiplication of works of art available to every one of us, superadded to the conflicting tastes and odors and sights of the urban environment that bombard our senses. Ours is a culture based on excess, on overproduction; the result is a steady loss of sharpness in our sensory experience. All the conditions of modern life - its material plenitude, its sheer crowdedness - conjoin to dull our sensory faculties. And it is in the light of the condition of our senses, our capacities (rather than those of another age), that the task of the critic must be assessed. What is important now is to recover our senses. We must learn to see more, to hear more, to feel more. $\ll^{202}$

Genau darum geht es mir hier. Mit Sontag bewusster zu hören und zu fühlen. Um dann im Wittgensteinschen Sinne zu zeigen, um anschließend mit Scott zu fragen: Fühlst Du das auch?

\section{Bewusstes Hören, Hören mit Ziel}

Im Nachdenken über Sontags »hear more« ist mir erst das Ausmaß bewusst geworden, mit dem ich inzwischen auf dem iPod unterwegs oder im Konzert oder zu Hause, während ich arbeite, Musik nur mit halber Aufmerksamkeit höre. Wie oft dies im Alltag der Fall ist. Das provozierte mich noch viel mehr. Denn wenn ich doch heutzutage mit dem Drücken weniger Tasten die Auswahl zwischen gefühlt unendlich vielen faszinierenden Musiken habe. Viel mehr, als Sontag schon bei Begriffen wie Exzess und Überproduktion vor Augen hat. Viel mehr aber selbst noch, als Gerhard Schulze Anfang der 1990er Jahre erahnen konnte, als er Die Erlebnisgesellschaft an die Diagnose einer »Gesellschaftsbildung durch Überfluß« knüpft. ${ }^{203}$ Und 
persönlich tatsächlich auch seit jeher einen breit diversifizierten Musikgeschmack pflege: Warum kehre ich als Hörer dann doch immer wieder zum Klavierquintett zurück? Ja, warum eigentlich? Schließlich hat man nur endlich Zeit zur Verfügung. Und davon auch nur einen deprimierend kleinen Teil zum Hören von Musik. Irgendetwas muss mich motivieren, immer wieder ausgerechnet zum Klavierquintett zu greifen.

So habe ich mir das Suchen nach einer Antwort nach dem »Warum Klavierquintett?« zum Anlass genommen, mich bewusst wieder auf das Hören überhaupt zu konzentrieren. Und dies eben nicht für ein oder zwei Gelegenheiten für ein oder zwei Stunden. Sondern gezielt als längeres Projekt. Allein die für dieses Buch näher betrachteten Werke und Darbietungen summieren sich auf über 50 Stunden Musik. Voneinander oft sehr verschiedene und in sich ausnehmend heterogene Musik. So ist das Folgende in gewisser Weise auch Dokument eines Selbstversuchs in »close attention «, wie es Lawrence Kramer nennt. ${ }^{204}$ Und zugleich Selbstversuch im Selbstverstehen im Sinne Georg W. Bertrams:

»Was der Mensch ist, ist er immer auch dadurch, dass er Stellung nimmt, und zwar zu sich [...] [Menschen] entwickeln durch ihre Auseinandersetzung mit Kunstwerken Verständnisse von sich und bestimmen damit, was sie als Menschen sind. $\ll^{205}$

Ein Selbstversuch, den das interessiert, was Stefan Zwinggi »Musik als affektive Selbstverständigung« genannt hat. ${ }^{206}$ Und Matthias Vogel, auf Populäre Musik bezogen, beschreibt als »Entwicklung und Transformation der personalen Identität«. ${ }^{207}$ Ein Nachdenken darüber, dass in meinem Fall von den eingangs zitierten Fragen angestoßen wurde und ausgeht, die Ben Ratliff in seiner Studie Every Song Ever, Twenty Ways to Listen to Music Now aufgeworfen hat:

»Now we can hear nearly everything, almost whenever, almost wherever, often for free: most of the history of Western music and a lot of the rest. We know all that music is there. Some of us know, roughly, how to encounter a lot of it. But once we hear it, how can we allow ourselves to make sense of it? [...] The most significant progress in the recent history of music has to do with listening. How we listen to music could be, for perhaps the first time in centuries, every bit as important to its history and evolution [...]. How are we going to access it, and 
how can we listen to it with purpose - meaning, how can we pay just enough attention to it so that it could change our lives? «208

»Listen to it with purpose« in Ratliffs Sinne, ist also die Aufgabe, die ich mir als Selbstversuch gestellt habe: Wenn ich mir Zeit nähme und bewusst hinhörte, ob ich wohl identifizieren könnte, was mich als Hörer an diesem Genre reizt? Und warum? Nicht an ein, zwei oder drei Arbeiten oder Performances. Und sicherlich auch nicht an allem, was innerhalb dieses Genres je gemacht worden ist. Aber doch in auffallend weiten Teilen? Bei welchen musikalischen Situationen kann ich eigentlich fragen: Fühlst Du das auch? Und bei welchen ist es mir wirklich wichtig? Bei welchen will ich das überhaupt? Welche schaffen es, ob ich will oder nicht?

\section{Alternative Musikgeschichte}

Schaut man auf die Enge des Kernrepertoires, auf die skizzierte Aufmerksamkeitsverteilung im Falle des Klavierquintetts in den Leitmedien Klassischer Musik, auf die geballte Kompetenz, die mit ihrer Autorität hinter diesem Narrativ steht, könnte man meinen, links und rechts davon gäbe es nichts interessantes an Musikgeschichte zu holen. Als Musikgeschichte zu erzählen. Das ist nicht nur langweilig. Sondern vor allem falsch. Den Weg über »emotional impact« zu gehen ist also keineswegs eine Notlösung, um einen über den engen Status quo hinausgehenden historiographischen $\mathrm{Zu}$ griff auf den Reichtum der Geschichte und Gegenwart dieses Genres zu finden.

Nehmen wir als Beispiel für einen solchen möglichen Ansatzpunkt den vorhin diskutierten Auszug der Videos mit den höchsten Klickzahlen aus der Trefferliste »Piano Quintet« bei YouTube. Der so überdeutlich das Kernrepertoire der Leitmedien Klassischer Musik als das von den Streamingnutzern dieser Plattform auch bevorzugt gewählte Repertoire bestätigte. Allerdings ist die Liste etwas verfälscht. Denn sie weist nur Videos von Musik aus, die als Klavierquintett gekennzeichnet ist. Das trifft aber nicht auf alle einschlägige Musik zu. So z.B. nicht auf »Advantage Points« vom Album Chambers (2015, Gentle Threat Gentle018CD), komponiert von Chilly Gonzales und eingespielt gemeinsam mit dem Kaiser Quartett. Dieses Stück ist für klassisches Klavierquintett komponiert. Wie 
das ganze Album. Und »Advantage Points« kommt auf gut $~ 580.000$ Streams. ${ }^{209}$ Das wäre Platz 3 in der Trefferliste »Piano Quintet«, direkt nach zwei Mal Schubert und vor allen anderen. Platz 3!

Das Stück »Advantage Points« wie das ganze Album Chambers gehören zum Genre Neoklassik. Neoklassik steht als jüngste Variante in einer langen Popularisierungstradition Klassischer Musik. ${ }^{210}$ Als Genrebezeichnung setzte sich der Begriff in den 2010er Jahren durch. Es gibt dabei gewisse Überschneidungen mit dem zeitlich parallelen, in Künstlern wie Musik aber nur eingeschränkt überlappenden Diskurs um das Genre Indie Classical. ${ }^{211}$ Während Neoklassik deutlich Richtung Pop- und Filmmusik neigt, hat Indie Classical weit mehr mit junger, aber popkulturinformierter Neuer Musik zu tun. Dem ungeachtet verbindet jedoch beides als Marketing- und Kommunikationsstrategie das Ziel, Aufmerksamkeit für Musik abseits des Mainstreams Klassischer Musik zu gewinnen.

Neoklassik steht inzwischen freilich im Kontext der Bemühungen der Leitmedien Klassischer Musik selbst, insbesondere der Musikindustrie und der diese prägenden Majorlabels, neue, vor allem jüngere Hörerschichten überhaupt für den Bereich Klassischer Musik zu interessieren. Jene, die nicht ohnehin über die Bildungsbürgerschiene kommen und vergleichsweise leicht für ein kernrepertoireorientiertes Angebot zu interessieren sind. Aber dafür vielleicht sphärische Filmmusik mögen. Oder meditative Klaviermusik. Oder stark repetitive elektronische Clubmusik.

Neoklassik sucht in seinen Aufführungsstätten gleichermaßen die Respektabilität renommierte Konzerthäuser und die Wildheit angesagter Clubs, das Prestige traditionsreicher Klassiklabels und den Flair progressiver Indielabels. Neben neuen Werken wird remixed und gesampled, recomposed und von DJs aufgelegt. Das eigentliche Zuhause dieser Musik ist jedoch die Welt der userkuratierten Playlists in Streamingportalen. Chilly Gonzales, seines Zeichens immerhin Grammy-Gewinner für ein Crossover-Projekt mit dem französischen Elektronik-Duo Daft Punk, steht in einer langen Reihe von Namen. Neben Nils Frahm, Ólafur Arnalds, Joep Beving, Max Richter, Sven Helbig, Ludovico Einaudi, Jóhann Jóhannsson, Hauschka, Dustin O'Halloran, Grandbrothers oder Francesco Tristano (wie in der Klassik generell üblich, finden sich selbst in ihrer heutigen Popvariante, der Neoklassik, nur ausnahmsweise Frauen wie Anna Meredith oder Micachu/ Mica Levi). Angenehm auftretende Hipstermänner. Angenehm auftretende 
Musik. Musik, die wie die anderen vollbärtigen Kreuzberger Papas klingt, die ich samstags in Berlin beim Vater-Kind-Sport treffe.

Die Sache hat einen Markt. Hübsch. Meditativ. Repetitiv. Zeitgemäß inszeniert. Dass sich die Sache oft verhält wie der New Age der 1970er und 1980er Jahre zu seinen Auslösern, insbesondere den lyrischen Passagen in den Soloimprovisationen des Jazzpianisten Keith Jarrett und Teilen der Minimal Music, ohne Interesse an den Details und den Ausdruckskontrasten, aber auch der Virtuosität und den Widerständigkeiten der Vorbilder, stört die Zuneigung des Publikums nicht. Mitunter klingt Neoklassik wie die Bonusinstrumentalversionen, die sich gerne als Dreingabe auf Singles befanden, als man noch auf Vinyl und dann CD Singles veröffentlichte. Vor allem aber scheinen alle Michael Nymans Soundtrack zu The Piano und Yann Thiersens Score zu Die fabelhafte Welt der Amélie zu lieben. Ohne freilich im Dialog mit deren kinematographischer Ästhetik, deren eindringlichen Drehbüchern und deren beeindruckend spielenden Besetzungen ihre Atmosphärik entfalten zu können.

Das Klavier ist entsprechend das zentrale Instrument des Genres. Und damit ist der Weg nicht weit zum Format des Klavierquintetts. Insbesondere, wenn man bedenkt, dass viel dieser Musik sich an Emotionen wie Traurigkeit, Melancholie und Einsamkeit abarbeitet. Und daher die Sehnsucht nach Streichern groß ist. Die Synthese des Klavierquintetts liegt nicht fern. Alben der Neoklassik operieren daher immer wieder nahe der Klangspezifik des Klavierquintetts. Klassische Platten des Genres wie Ludovico Einaudis Eden Roc (1999, BMG Ricordi 74321 707172), Ólafur Arnalds' Eulogy for Evolution (2007, Erased Tapes Records ERATP4CD = 2017, Erased Tapes Records ERATP101CD - Remastered durch Nils Frahm) oder Max Richters Infra (2010, FatCat Records CD13 $11=2017$, Deutsche Grammophon 4797006 - Reissue). Nur, dass diese Alben zwar an vielen Stellen die Idiomatik des Klavierquintetts suchen und andeuten, aber die Besetzung nicht strikt achten - Einaudi besetzt einen Kontrabass (und bisweilen ein Duduk), Arnalds eine zusätzliche Violine (und gen Ende noch traditionelle Rockbandinstrumentation) und Richter ein Cello mehr. Arnalds und Richter nutzen zudem alle möglichen Elektronika und alle drei arbeiten mit Postproduktionseffekten. Vor allem aber bleibt der Klavierquintettbezug stets Nebensache. Oft nur klangliche Allusion. Es geht nicht zuvorderst um das Ensembleformat oder gar seine spezifische Tradition. 
Immer steht das Instrument des Komponisten, das Klavier, im Zentrum, der Alben. Aber die Allusion zum Klavierquintett ist doch stets gegenwärtig.

Das ist bei Gonzales' Chambers anders. Er inszeniert tatsächlich das klassische akustische Klavierquintett als solches. Ohne Schnickschnack. Ohne Extras. Nur eben hier als Popband. Kurze, meist prägnante Nummern in Popformen. Künstler wie Einaudi, Arnalds und Richter operieren irgendwo zwischen Ambient und Easy Listening, New Age und Minimal Music. Oft sehr langsame, sphärische Musik. "Advantage Points« oder etwa »Green's Leaves« von Chambers sind anders. Sie sind charmante, frohgestimmte Popmusik, die auch gut auf Indieplatten wie jenen von The Style Council gepasst hätten und nicht weit weg sind von The Smiths - nur ohne den doppelten Boden der bitterbösen Lyrics von Morrissey.

Gonzales' Chambers ist ziemlich einfache Musik, in Komposition wie Arrangement. Was innerhalb der Neoklassik in der Symbiose von populären Idiomen und klassischen Spielweisen möglich wäre für Klavierkammermusik, wenn die Arrangements der spieltechnischen und interpretatorischen Qualität exzellenter klassischer Interpreten gerecht werden, hat freilich schon vor Jahren ein von Sven Helbig produziertes Album für Klavierquartett vorgeführt. Popsongs des Fauré Quartett (2009, Deutsche Grammophon 476 361-0). Voll an B-Seiten und versteckten Albumtracks etablierter, oft aber auch nur in ihren jeweiligen Genres prominenter Popkünstler. Dieser Tonträger klingt auch nach 10 Jahren noch frisch, verspielt, detailreich, überraschend, virtuos, fantasievoll, einnehmend. Anspruchsvoll, interessant und zugänglich zugleich. Bei allem Charme muss Gonzales' Chambers gegenüber dieser Musik für Klavierquartett flach wirken. Aber nichtsdestotrotz markiert sein Album ein Extrem im Spielraum des Klavierquintetts heutzutage. Der so viel größer ist als das omnipräsente Kernrepertoire. Einen Spielraum, der eben auch Resonanz in Größenordnungen populärer Musik provozieren kann. Wie eben bei »Advantage Points« bei YouTube.

Die Sache hat allerdings eine Konsequenz. Diese lässt sich z.B. am Klavierquintett Alba de Los Caminos von Antón García Abril erläutern, eines, das ich später wieder aufgreifen werde. Es stammt aus dem Jahr 2007. Verfasst von einem über 70jährigen Spanier. Provokant könnte man sagen, es ist die Art von Musik, von der die mit dem Flair der Jugend flirtende Neoklassik träumt, sie zu schreiben, in ihrem Versprechen, Hörer mit einer primären Sozialisation in populärer Musik dort abzuholen und für 
Traditionen Klassischer Musik zu gewinnen. Traditionen wie das Klavierquintett. Die Sache wird zum Problem, weil Stücken wie Alba de Los Caminos kein Raum für Aufmerksamkeit verbleibt. Wenn nämlich die Intellektuellen nur die aggressive Moderne der Klavierquintette eines Xenakis, Messiaen oder Carter und die eher depressiv-sphärische Variante eines Schnittke oder Feldman, Henze oder Adès gelten lassen und überhaupt beachten. Und zugleich die großen, prestigeträchtigen Plattenfirmen und Interpreten sowie der sie begleitende Musikjournalismus umgekehrt nur Klassiker berühmter Namen im Blick haben. Oder eben Neoklassik. Carter und Schnittke, Feldman und Henze, Adès und Chilly Gonzales: Sie alle finden gleichberechtigt ihren Platz in diesem Buch. Neben Schumann und Brahms. Faszinierend auf ihre Art. Völlig gegensätzlich in ihren ästhetischen Zielen und kompositorischen Mitteln, spieltechnischen Voraussetzungen und Wissensanforderungen an den Hörer. Was aufgerieben wird zwischen alldem ist aber der Platz für etwas, das im Englischen middlebrow genannt wird. Und sich in meinem eigenen Zugriff als Hörer auf die Gegenwart dieses Genres völlig selbstverständlich gleichberechtigt daneben gesellt. Das Zugängliche mit Anspruch. Das Anspruchsvolle, das den Zugang nicht zu einem extrem aufwändigen Unterfangen macht. Musik wie den New Yorker zu lesen oder Quality-TV-Serien zu schauen wie Breaking Bad oder The Wire. Alba de Los Caminos ist genau dies: middlebrow. Wie die in diesem Buch aufgegriffenen, wieder im Vergleich miteinander jeweils völlig anders gearteten zeitgenössischen Stücke von Bent Sørensen (2014) oder Jefferson Friedman (2014), Nikolai Kapustin (1998) oder Morten Gaathaug (2005), Vijay Iyer $(2005,2014)$ oder Ian Venables (1995), Fazil Say (2017) oder Albin Fries $(2007,2008)$ auch. Der Bereich des middlebrow ist nicht nur groß. Er ist heterogen. Er ist spannend. Er ist Kunst. Und er ist verdammt schwer zu erkunden.

Dieses Fallbeispiel ist keine Ausnahme. Ohne auf die Kategorie des »emotional impact« verwiesen zu sein, lassen sich viele vergleichbar produktive Ansatzpunkte finden, vom Klavierquintett zu erzählen, ohne bei Schubert, Schumann und Brahms zu verharren - oder im Zusammenspiel mit ihnen. So könnte man darüber berichten, bei wie vielen Komponisten von Brahms und John Alden Carpenter über Hans Werner Henze und Konstantia Gourzi bis Bent Sørensen und Fazil Say das jeweilige Klavierquintett in eine komplizierte, aber instruktive Fassungsgeschichte gehört. ${ }^{212}$ Weil man nie zufrieden mit der Gestalt der Sache war (Brahms, Carpenter). 
Weil das Klavierquintett als Vorstufe eines größeren Werkes diente (Henze). Oder weil absichtsvoll verschiedene Varianten desselben musikalischen Materials nebeneinander gestellt und gleichberechtigt veröffentlicht wurden (Sørensen, Gourzi, Say). Und all dies erlaubt, Varianten, manchmal auch nur noch Spuren der gleichen Musik in verschiedenen Gewändern zu hören. Was etwa gestattet, im direkten Vergleich viel über die Eigenart des Klavierquintetts als Genre zu lernen.

Oder man könnte seine Aufmerksamkeit darauf richten, die angesprochene Indie-Grass-Roots-Bewegung zu beschreiben, in ihren Akteuren und Zielen, ihrer Strategie und Resonanz. Oder sich einmal systematisch durch all die No-Name-Komponisten arbeiten, die jetzt, hier und heute, Klavierquintette schreiben und diese auf Plattformen wie SoundCloud oder YouTube zum Hören anbieten, erkunden, was für Musik das ist und wer sie warum macht, ohne jede realistische Chance auf ein größeres ökonomisches oder kritisches Echo.

Oder man könnte sich mit extremen Fällen und ihrem historiographischen Potential beschäftigen. Etwa Wilhelm Furtwänglers krudem Klavierquintett in C-Dur WF 112 (1912-35) von gut 80 Minuten Aufführungsdauer. ${ }^{213}$ Das ein Engagement jenseits des Werks belohnt. Nach seinem 1909 komponierten und im November 1910 in Breslau uraufgeführten Te Deum hatte Wilhelm Furtwängler für zweieinhalb Jahrzehnte kein fertiges Werk mehr vorgelegt. In jenen Jahren erarbeite er sich stattdessen den Ruf des neben Arturo Toscanini wohl bedeutendsten Dirigenten seiner Zeit, ab 1922 insbesondere am Pult der Berliner Philharmoniker. Ein Ruf, der bis heute nachhallt. Der Fokus auf das Dirigieren änderte sich erst, als der sogenannte »Fall Hindemith« Furtwängler Ende 1934 vorrübergehend aus dem öffentlichen Musikleben zwang. Furtwängler war öffentlich für Paul Hindemith eingetreten, der in den Monaten zuvor unter erheblichen Druck der nationalsozialistischen Kulturpolitik und Presse geraten war. Erst ab diesem Zeitpunkt, nach einer Pause von immerhin gut einem Vierteljahrhundert, beschäftigte sich Furtwängler wieder mit dem Komponieren, jedenfalls in einer Ernsthaftigkeit und Konsequenz, die veröffentlichungsfähige Werke entstehen ließ. Die ersten beiden der nun entstehenden auffallend zahlreichen späten Schöpfungen waren zwei Kammermusikstücke: die Violinsonate Nr. 1 d-Moll und eben das Klavierquintett C-Dur, die beide 1935 fertiggestellt wurden. Sie griffen Vorarbeiten auf, die zum Teil mehr als 20 Jahre zurückreichten. Letzteres ein Stück, das kein Ende nimmt. Ohne klare 
Richtung. Und vor allem anderen laut. Dabei wirkt es in allem bitterernst. Ganz wie die Bildaufnahmen Furtwänglers und seine ästhetischen Schriften. Mich lässt es ratlos zurück. Bis auf einen kurzen Augenblick, an dem ich immer lachen muss. Ob diese Stelle wohl so gemeint war? Mitten im zweiten Satz wird vom Klavier wie aus dem Nichts der »Walkürenritt« aus Richard Wagners Oper Die Walküre zitiert. ${ }^{214}$ Es sind nur wenige Sekunden. Aber es drückt in dieser einen Geste aus, was diese Musik sein will: Ring des Nibelungen. Maximal große Geste. Dass dieses Zitat dabei wenigstens für meine Ohren leider der prägnanteste Augenblick in der ganzen Partitur ist, illustriert jedoch, voran all dies scheitert. Das ist komisch. Vielleicht unfreiwillig komisch. Aber dafür doch ziemlich. Es lohnt sich, dass Werk allein für diese Passage anzuhören. Das zu tun aber, ist musikhistoriographisch in anderer Hinsicht viel interessanter: Denn es ist durchaus produktiv, der engen Verzahnung von Dirigent und Komponist nachzugehen, die Furtwängler selbst oft hervorgehoben hat. Roman Brotbeck hat darauf hingewiesen, dass

"gewisse Momente, die beim Komponisten [Furtwängler] als Defizite in Erscheinung treten, sowohl zu den spezifischen Qualitäten des Dirigenten gehören als auch dessen historische Grenzen anzeigen. $\ll^{215}$

Angesicht des unstrittigen Umstandes, dass Furtwängler ein bedeutender, origineller und einflussreicher Dirigent war, als Komponist all dies jedoch nicht verkörperte, ist der historiographische Mehrwert ungleich höher, wenn man den Komponisten Furtwängler nutzt, um dem Dirigenten Furtwängler näher zu kommen. Und die auf diese Weise zu gewinnenden Erkenntnisse sind tatsächlich weiterführend. So scheinen etwa in den ausführlichen Tempobeschreibungen in Furtwänglers Partituren der Art wie »Sehr allmählich das Tempo steigern«, »Kaum merklich überleiten« oder »Immer dasselbe fieberhaft vorwärtsdrängende Tempo (ohne eigentlich schneller zu werden)« Interpretationscharakteristika des Dirigenten durch. Nicht umsonst waren das Tempo rubato oder die Preisgabe großformaler Tempodispositionen zugunsten momentaner Spannung Hauptkritik- bzw. Bewunderungspunkte am Dirigierstil Furtwänglers, je nach Position des Betrachters. Auch Charakteristika wie Pathos, Emphase und Streben nach Monumentalität finden sich hier wie dort. Was jedoch dem Dirigenten zur Individualität verhilft, scheint dem Komponisten zu schaden, da er hier nicht mit einer 
dramaturgisch wirkungsvoll gestalteten Vorlage arbeiten und diese um seinen Interpretationsansatz ergänzen kann, sondern auf diesen vollständig verwiesen bleibt. Das jedoch erweist sich als Zuviel des Guten. Ob Furtwängler dies wohl selbst ahnte, als er feststellte: »Die Gefahr beim monumentalen Kunstwerk ist die Ermüdung « $?^{216}$

All dies sind nur Beispiele für Angebote, die das Genre des Klavierquintetts dem Musikhistoriker macht. Um über die Enge des Kernrepertoires hinauszuschauen. Musikgeschichte kann man dabei auf sehr viele Arten schreiben. Als kompositorische Innovationsgeschichte etwa. Oder als Geschichte von Einflüssen, der Übernahmen, aber insbesondere auch dem, was in Auseinandersetzung mit Bestehendem jeweils gerade nicht übernommen wurde. Man kann mittels Biographik vorgehen. Oder von technisch-mediale Entwicklungen berichten. Ereignisse wie Konzerte oder Tonaufnahmen in den Blick nehmen. Oder Erinnerungsorte aufsuchen. Eine Erzählung entwerfen, für die Relevanz durch die Quantität von Resonanz begründet wird, in Gestalt von Verkaufszahlen oder Kritikerreaktionen oder politischer Wirkung etwa. Oder in Epochen oder Ideen denken. Sich an Genres, Stilen oder Subkulturen orientieren. Oder Musikgeschichte entlang von Dokumenten, Institutionen oder Werken erkunden. Dies sind nur einige der gangbaren und häufig gewählten Wege. Jede Herangehensweise rückt andere Aspekte des Gegenstands in den Vordergrund. Lässt einen eine andere Perspektive auf ihn einnehmen. Und es ist der Vergleich pointiert eingenommener und konsequent durchgeführter Perspektiven, aus dem ich jedenfalls oft am meisten Gewinn ziehe. Die Enge des Kernrepertoires verstellt den Blick darauf, dass all dies auch mit dem Klavierquintett geht und lohnt.

Schaue ich freilich auf meinen iPod, sehe ich noch eine ganz andere Musikgeschichte. Eine, die selten vorkommt und die ich selbst zuvor auch wenig reflektiert habe. Die mehr oder weniger einfach passiert ist. Eine Musikgeschichte in »emotional impacts«. Eine persönliche Musikgeschichte zwar. Aber doch eine Musikgeschichte.

Dass ich diesen Selbstversuch aufschreibe, hat daher nicht nur mit dem zu tun, was Haruki Murakami so eindrücklich in der Einleitung seines Buches Wovon ich rede, wenn ich vom Laufen rede schildert: »[...], ich kann viele Dinge nur begreifen, indem ich meine Gedanken zu Papier bringe. Ich muss verfassen, um zu erfassen. $\ll^{217}$ Ich spüre der Selbstbeobachtung vielmehr mit einer bestimmten Stoßrichtung nach. Ganz wie im ersten 
Kapitel mit der Beschreibung der Leitmedien Klassischer Musik wird versucht, zu erschließen und nachzuvollziehen, was für ein Zugang zu Musikgeschichte durch die jeweilige Zugangsweise geformt wird. Welche Mechanismen am Werk sind. Mit welchen Konsequenzen. Nur während es mir im ersten Kapitel um das Tun anderer ging, geht es mir hier um mein eigenes Tun. Zu erkunden, wie jenseits gängiger historiographischer Mechanismen und ihrer Ausgangs- und Ansatzpunkte letztlich etwas entsteht, das man mit Elijah Wald >Alternative Musikgeschichte` nennen kann. ${ }^{218}$ Auswählen aber nicht wie im Fall von Wald über das alternative, vermeintlich objektivierbare Kriterium dessen, was zu einer gegebenen historischen Zeit jeweils quantitativ gesehen das Populärste war. Sondern aufgrund eines jahrzehntelangen Erkundens eines jeweiligen Repertoires entlang dessen, was »emotional impact« auf mich entfaltet. Meine >Alternative Musikgeschichte des Klavierquintetts. So wie ich für mich in meinem alltäglichen Umgang mit Musik für ganz unterschiedliche musikalische Praxen >Alternative Musikgeschichten` geschrieben habe. In meinem Hörverhalten lebe. Für das Broadway-Musicals. Oder den britischen Indierock. Oder die amerikanische Gegenwartsoper. Oder das Indiefolk. Oder des zeitgenössischen Jazz. Um andere Ordner auf meinem iPod zu nennen. Andere Auswahlen, die ich treffe.

Guyers Kategorie des »emotional impact« wurde deswegen bestimmend für mein Nachdenken, weil sich mein Auswahlhandeln als einfacher Hörer bei genauer Selbstbeobachtung als eben von »emotional impact« geleitet herausstellte. Nicht immer. Aber eben doch oft. So oft, dass mich interessierte, dieses Auswahlhandeln besser zu verstehen. So wie im ersten Kapitel die Lenkungswirkung von Markennamen auf das Auswahlhandeln.

Dabei zeigte sich mehrerlei recht schnell: »Emotional impact« wird nicht durch Namen von Komponisten oder Interpreten, Verlagen oder Plattenfirmen getriggert. Den zentralen Akteuren des ersten Kapitels also. Das Auswahlhandeln bleibt zudem weitgehend unbeeinflusst von Fragen kulturellen und sozialen Kapitals in Pierre Bourdieus Sinne. ${ }^{219}$ Allein schon schlicht deswegen, weil solch kulturelles und soziales Kapital nach meiner langjährigen Erfahrung von Wissenschaftskollegen bis Social Media mit einer solch randständigen musikalischen Praxis abseits der Markennamen wie dem Klavierquintett allenfalls ausnahmsweise zu generieren ist. Praktisch ist das aber sehr selten. Aus diesem Grund teile ich in diesem Fall auch nicht das generelle Misstrauen der Emotionsforschung gegenüber 
Selbstbeobachtungsberichten über »emotional impact«, das stark auf den Einfluss des sozialen Befragungsrahmens abstellt - neben der mutmaßlichen Unfähigkeit des Beschreibenden, nicht zwischen eigener Emotion und Emotion in der Musik differenzieren zu können, eine Befürchtung, die mir hier keine Sorge bereitet. ${ }^{220}$

Aber nicht nur soziale Faktoren haben sich als vernachlässigbar gezeigt. Auch kompositorische Gesamtarchitekturen treten entgegen Adornos entschiedener Forderung als Faktor deutlich in den Hintergrund, zugunsten eben von oft erstaunlich kurzen musikalischen Situationen. ${ }^{221}$ Deren »emotional impact« in diesem Fall schließlich auch nicht von außermusikalischen Faktoren provoziert und gelenkt wurde, die ich natürlich auch kenne, aber eben nicht an dieser Stelle. D.h., dass »emotional impact« hier nicht ausgelöst oder verstärkt oder geformt wurde etwa durch die Verbindung der Musik mit herausgehobenen autobiographischen Momenten oder über die Bedeutung der Musik für eine Gruppe und meine Zugehörigkeit zu dieser und damit letztlich über meine Identifikation mit ihr. ${ }^{222}$ Schon, weil in meiner Welt - Freunde und Familie, Fachkollegen, Studierende, Social Media usw. - sich wirklich niemand für das Thema begeistert. Das Hören von Klavierquintetten ist in meinem Fall also tatsächlich ein ziemlich privates Privatvergnügen. Anders als in den meisten anderen Musikbereichen, die mich involvieren. Diese Spezifik des Klavierquintetts für mich erlaubt es mir aber wiederum in der Selbstbeobachtung, gezielter die Frage nach den musikalischen Situationen zu stellen, die hier einmal ganz klar für den »emotional impact« verantwortlich zu machen sind. Ein zentraler Grund für die Auswahl ausgerechnet des Klavierquintetts als Fallbeispiel.

Diese Selbstbeobachtungen lege ich später im abschließenden Kapitel offen. ${ }^{223}$ Und spüre ihnen nach. Nach, um zu erkunden, wie hierdurch eine >Alternative Musikgeschichte $<$ des Klavierquintetts entsteht. So ist jenes Kapitel dann ein Unternehmen ganz im Geiste dessen, was Albrecht Riethmüller vor einem Vierteljahrhundert in abstracto als Ansatz für >Alternative Musikgeschichte< beschrieben hat:

»Was änderte sich, wenn wir uns einigten, die Namen der Komponisten nicht mehr zu verwenden, sondern nur noch die Musikwerke so bezeichneten, wie wir auch von Büchern des Alten Testaments nur Titel, keine Verfasser kennen und wie das Buch Hiob nicht von Hiob und das Seikilos-Lied nicht von Seikolos stammt: Namen für Werke, keine Namen für Autoren. [...] Durch die Privation 
der Namen zerbräche die fraglose Gleichung Genie = Verfassername, zerbräche unsere von diesen Wörtern bzw. Namen bestimmte Sicht der Musik und von Teilen ihrer Geschichte. Man müsste sich dann mit der Sache selbst abgeben $[\ldots] \ll^{224}$

Ich kontrastiere mit den Teilen dieses Buches also diametral entgegengesetzte Zugänge auf denselben Gegenstand miteinander. Mit Absicht. Auf Augenhöhe. Um im Vergleich sichtbar zu machen, welch unterschiedliche Musikgeschichten des Klavierquintetts sich auf diese Weise ergeben. Und was für einen Unterschied macht es hier! Denn während das erste Kapitel mit dem Klavierquintett eine musikgeschichtlich ziemlich nebensächliche Angelegenheit beschreibt und erklärt, warum ihm dieser Charakter des Nebensächlichen anhaftet, offenbart das abschließende Kapitel einen ungemein reichen Strang an Musikgeschichte, eine Hauptsache sozusagen. Für mich.

\section{MIXTAPE: EINE IMAGINIERTE, VIRTUELLE Ausstellung MUSiKALISCHER SituAtionen}

Die Auswahl an Musik, über die ich sogleich sprechen werde, ist bewusst und ausdrücklich subjektiv gemeint. Sie kommt ohne jeden Anspruch auf Verallgemeinerbarkeit, historische Korrektheit, kompositions- und/oder interpretationshistorische oder gar universelle ästhetische Relevanz. Dies ist kein Kanon. Es geht nicht um Meisterwerke und ihre Schlüsselstellen. Nicht um ihre Rolle für die Entwicklung des Genres. Das Folgende steht einem Mixtape mit Lieblingsliedern ungleich näher. Ich trage schlicht jene musikalischen Situationen zusammen, auf die ich verweise, d.h. z.B. in Noten zeige oder als Tonaufnahme vorspiele oder in Worten beschreibe, wenn ich gefragt werde, warum ich mich für das Klavierquintett als Genre nicht nur als eine wissenschaftlich ertragreiche Praxis interessiere. D.h. so, wie ich das Genre im ersten Teil dieses Buchs argumentativ genutzt habe, um hierüber eine soziale Praxis zu beschreiben. Sondern abweichend zu vielen anderen meiner wissenschaftlichen Arbeitsfelder tatsächlich seit langem und immer noch oft höre. Daher ist Musikgeschichtsschreibung eigentlich das falsche Wort für mein Anliegen hier. Denn genauer gesagt wird nicht geschrieben, sondern kuratiert, wie schon die Analogie zum 
Mixtape nahelegt. ${ }^{225}$ Getreu Hans Ulrich Gumbrechts Definition des Kuratierens:

»Daneben - und wohl vor allem - bezieht sich das Verb >kuratieren Entfaltung eines Themas oder eines Phänomens in all seinen Dimensionen und auf verschiedenen organisatorischen Ebenen: durch die Auswahl, das grundlegende Verständnis und die spezifische Auslegung dieses Themas; durch die kurzen Texte, mit denen die Gegenstände der Ausstellung für ihre Besucher identifiziert und in den weiteren Kontext einer Konzeption eingeordnet werden; aber auch durch die Art und Weise, wie der Kurator die Ausstellungsgegenstände im zur Verfügung stehenden Raum verteilt und zueinander in Beziehung setzt.. ${ }^{226}$

Kuratieren in all der entschiedenen Subjektivität, die Dorothea von Hantelmann als Kerncharakteristikum des Kurators ausmacht: »The curator emerges as a figure who exemplarily constitutes himself or herself through aesthetic choices $[\ldots] . \ll^{227}$

Es geht mir also darum, zu erkunden, wie diese >Alternative Musikgeschichte des Klavierquintetts entsteht, indem ich jene musikalischen Situationen offenlege, die bei mir »emotional impact» hervorrufen. Und hierdurch wiederum eben jener >Alternativen Musikgeschichte< Kontur verleihen, ja diese überhaupt erst als solche entstehen lassen, die der Ordner »Klavierquintette« auf meinem iPod darstellt. Weil das Vorhandensein einer solchen musikalischen Situation in diesem Fall der einzige Grund für die Aufnahme einer bestimmten Interpretation eines bestimmten Werks in eben diesen Ordner ist. Das ist von Hantelmanns ıästhetische Auswahlı, so wie sie hier getroffen wird. Diese Auswahl wird dann in Gumbrechts Sinne ausstellungsgleich lediglich mit einer kurzen Beschreibung versehen. Mit Wittgenstein sollen diese Angaben einzig leisten, den Standort zu markieren - die situativen musikalischen Anlässe für »emotional impact« nämlich -, von dem ich aus vorschlage, den jeweiligen Ausstellungsgegenstand wie die imaginierte, virtuelle Ausstellung insgesamt als Zusammenhang zu betrachten, d.h. genauer: zu hören.

Die Gesamtheit dieser Ausstellungsgegenstände formt jene >Alternative Musikgeschichte` des Klavierquintetts, als die diese Musik über den Ordner »Klavierquintette« zusammen gehört wird. In dieser Zusammenstellung Gemeinsamkeiten und Unterschiede betont. Hinsichtlich dessen, was »emotional impact« verantwortet. Aber natürlich auch darüber hinaus. Eine sol- 
che Auswahl sieht je nach Anlass und Ziel des Kuratierens ganz unterschiedlich aus. Das ist keine Best-of-Liste. »Emotional impact« ist an dieser Stelle nur eine Option. Ein Zugangsmodus. Eine Perspektive auf die Sache. Alternative Blickwinkel, der mich an anderer Stelle beschäftigen, sind z.B. die Genderfrage, der Mangel an Frauen in diesem musikalischen Milieu, oder die Frage, was die besagte Indie-Grass-Roots-Bewegung wann, wo und warum an Repertoire erschließt. Jedes Kuratieren stellt Musik nebeneinander und setzt sie ins Verhältnis. Was in Relation zueinander wieder andere Gesichtspunkte betont. Manchmal überhaupt erst sichtbar macht. Ich kenne keinen objektiven Zugang zur Geschichte und Gegenwart des Genres. Keine neutrale Position. Nur offengelegte Gründe für den jeweiligen Blickwinkel. Verschiedene Blickrichtungen. Und Unterschiede in dem, was je nach Betrachterposition erkennbar werden kann und wird.

Mich interessiert hier hingegen nicht, warum die ausgewählten musikalischen Situationen psychisch, physiologisch oder neurologisch einen »emotional impact« auf mich haben. Warum ich körperlich einen Unterschied spüre. Warum es sich anders anfühlt - was es tut. Es mich berührt. Deswegen ist dieses Kapitel auch kein Beitrag zu eben diesen Bereichen der Emotionsforschung geworden. ${ }^{228}$

Mich interessiert an dieser Stelle gleichfalls nicht, ob die Komponistinnen und Komponisten der nachfolgenden Werke oder ihre musikalischen Interpretinnen und Interpreten intendiert haben, dass die musikalischen Situationen, die ich herausgreifen werde, einen besonderen »emotional impact« auf einen Hörer wie mich entfalten. Manche musikalische Situation ist klar so inszeniert. Manch andere nicht. Das ist mir zwar wichtig, im Blick zu halten. Weil es mitunter einen Unterschied macht. Ob es intendiert ist, scheint mir jedoch mit William K. Wimsatt und Monroe C. Beardsley, Roland Barthes und Michel Foucault sekundär. ${ }^{229}$ In diesem konkreten Fall sekundär, weil es die einschlägigen musikalischen Situationen für mich nicht hierarchisiert. Ob diese herausgehobene Wirkung auf mich oder $\mathrm{Mu}-$ sikhörer ganz allgemein nun von Komponistin oder Komponist, Interpretin oder Interpret intendiert war oder nicht, spielt letztlich keine Rolle. Der »emotional impact« ist für mich da, so oder so. Und ihn möchte ich benennen. Dafür werben, dies im Kontext von Musikgeschichtsschreibung offen zu tun. Und in Kermans Sinne gelten zu lassen. Dabei prüfen, ob ich eruieren kann, wo genau der »emotional impact« in dem zu verorten ist, was ich höre. Und ob ich dies so beschreiben kann, dass zumindest ich selbst mein 
eigenes ästhetisches Erleben dieser musikalischen Situationen in den Beschreibungen wiedererkenne. Damit ich überhaupt wagen kann, zu versuchen, dies jemand anderem zu zeigen. Und um Zeigen können im Wittgensteinschen Sinne geht es am Ende.

Deswegen interessiert an dieser Stelle schließlich ebenfalls nicht, wie viele Menschen diesen »emotional impact« am Ende teilen, heute oder in der Vergangenheit. Ich zeige nur auf etwas, das ich sehe. Und schaffe Raum für die Frage: Fühlst du das auch? Doch die Antwort ist nicht entscheidend. Mit Kant genügt, dass ich ästhetische Erfahrung und Urteil für teilbar halte. Sie muss es nicht empirisch sein. Die Antwort interessiert mich zwar. Aber sie spielt für den Ansatz selbst keine Rolle. Die Frage zu stellen, die Frage überhaupt zuzulassen, darauf kommt es mir an. Den Blick auf den »emotional impact« dieser musikalischen Situationen zu lenken. Und diese Perspektive in ihren persönlichen historiographischen Konsequenzen zu verfolgen. Um diese einmal nicht gleich wieder hinter dem Bemühen um Objektivier- und Verallgemeinerbarkeit verschwinden zu lassen. Hinter den Messdaten von Musikpsychologie und Neuroästhetik etwa und den Befragungsstatistiken der Musiksoziologie. ${ }^{230}$ Oder hinter den sich auf formale Aspekte konzentrierenden Analysestrategien in Musikwissenschaft und Musiktheorie. ${ }^{231}$ Oder hinter der Beschreibung der kulturellen Rahmenbedingungen und Einflussfaktoren auf mein ästhetisches Erleben, wie etwa im Sensory Turn insbesondere der Anthropologie, Ethnologie, Geschichts- und Kulturwissenschaften in den Vordergrund gerückt ist oder, der Musik oft am nächsten, in den Sound Studies. ${ }^{232}$ Um nur stellvertretend einige alternative, wissenschaftlich produktive Annäherungsweisen zu nennen. Sondern eben hierneben als Perspektive »emotional impact« in seiner ganzen Subjektivität zuzulassen. Zu erkunden, wo es hinführt, wenn man beachtet, wohin und worauf das, was in der Selbstbeobachtung hervortritt, in der jeweiligen Musik bezogen, ihr zugewandt ist. »Zugeeignet der Welt«, wie es in Maurice Merleau-Pontys Verteidigung dieser Position, seiner Phänomenologie der Wahrnehmung, heißt. ${ }^{233}$ Nicht um seiner selbst willen, sondern um in meinem Fall daran anschließend gerade hierüber eine >Alternative Musikgeschichte` des Klavierquintetts zu erzählen. Den entsprechenden Ordner auf meinem iPod überhaupt als solche zu erkennen und zu reflektieren. Denn genau eine solche entsteht hier nämlich: eine >Alternative Geschichte` des Klavierquintetts. Nicht etwa eine `bessere`, im Kontrast etwa zu der im ersten Kapitel skizzierten Art von Musikgeschichte - 
was erzählen $\mathrm{zu}$ wollen in jeder Hinsicht ein deprimierendes kritisches Unternehmen wäre, wie Bruno Latour gezeigt hat. ${ }^{234}$ Vielmehr wird lediglich eine parallel hierzu, aber eben nicht weniger real existierende Musikgeschichte offengelegt.

Ebenso beiläufig wie unvermeidlich entsteht mit dem hier gewählten kuratorischen Ansatz natürlich mit der anschließenden Sammlung an Beschreibungen ausgewählter musikalischer Situationen etwas, das Lydia Goehr mit kritischem Verve ein simaginiertes Museum musikalischer Werker genannt hat. ${ }^{235}$ Und nicht nur von Werken, sondern zugleich auch von bestimmten, als Tonaufnahmen materialisierten Interpretationen dieser Stücke, seien diese Aufführungen nun durch den Tonträger dokumentiert oder - was, wie Nicholas Cook beschrieben hat, die Regel ist - als Aufführung in der Postproduktion modifiziert, bisweilen über Schnitte überhaupt erst als kohärente Darbietung simuliert. ${ }^{236}$ Genau so wirkt das Verzeichnis zu Beginn des letzten Kapitels auf mich: Wie ein >imaginiertes Museum musikalischer Werker. Wie ein Ausstellungskatalog.

Aber: So folgenreich und limitiert das Werkkonzept in vielerlei Hinsicht sein mag. So sehr dies gleichermaßen für sein Pendant, das Sprechen von Referenzinterpretationen gilt, das Tonaufnahmen letztlich wie Werke behandelt. So sehr die Künstewissenschaften in einer endlosen Kette an »Turns« in den vergangenen Jahrzehnten andere Aspekte von Musik und Musikgeschichte in den Vordergrund gerückt haben oder hierfür genutzt werden können, von Aesthetic Turn, Spatial Turn, Material Turn, Body Turn, Linguistic Turn, Cultural Turn, Affective/Emotional Turn, Biographical Turn, Iconic/Pictorial Turn über Historic Turn, Hermeneutic Turn, Ecological Turn, Reflexive/Literary Turn, Mnemonic Turn, Neurological Turn, Postcolonial Turn, Translational Turn sowie Sociological Turn, Post-human Turn, Praxeological Turn, Interpretative Turn, Performative Turn, Sensory Turn, Ethical Turn, Realistic Turn bis zum wohl aktuellsten Turn, dem Digital Turn der Digital Humanities. ${ }^{237}$ Das virtuelle Museum, das so entsteht, hat ungeachtet aller aus dem einen oder anderen Blickwinkel sicher zurecht möglichen Einwände viel mit jedenfalls meiner Lebenswirklichkeit zu tun, meinem Umgang mit Musik - und sei es eben in der lapidaren Gestalt des besagten Ordners »Klavierquintette« auf meinem iPod. Ich mache nicht Spotify an und lasse mir per Zufallsmodus irgendwelche Musik vorspielen. Oder gehe zu Konzerten nach Location, gleichgültig, wer spielt und was. Ich wähle aus. Auch wenn ich weiß, dass es nur ein Ausschnitt bleibt: Ich 
baue mir so eine eigene Musikgeschichte des Klavierquintetts. Oder eben des Broadway-Musicals. Oder des britischen Indierocks. Oder der amerikanischen Gegenwartsoper. Oder des Indiefolk. Oder des zeitgenössischen Jazz.

Es mag ein imaginiertes, virtuelles Museum sein, das so entsteht. Und gewiss nicht der vollkommenste Bau zum Verstehen dieser Musik. Oder gar der einzig legitime Zugang. Oder der alleinig produktive. Aber es ist beileibe kein totes Konstrukt, lediglich unreflektiert einer überkommenen Art der Musikgeschichtsschreibung als Werkgeschichte geschuldet. Sondern ein sehr lebendiges, nämlich in meinem Alltag ungebrochen präsentes. Es ist ein imaginiertes, virtuelles Museum, wie es letztlich jeder für sich baut. Und zu dem man bei Gelegenheit anderen Zugang gewährt. So wie ich hier.

Wobei Goehrs Begriff des Museums hier eigentlich der falsche Ausdruck ist, so er zu viel Dauerhaftigkeit impliziert. Die Angelegenheit ist flüchtiger. Vergleichbar eher einer imaginierten, virtuellen Ausstellung, die temporär auf einen bestimmten Ausstellungszweck hin kuratiert, zusammengetragen und gehängt ist. Nicht von Wiederholbarkeit der Ausstellung ausgeht. Oder sich wichtiger nimmt als andere Ausstellungen im gleichen Museumsbau der Musikgeschichte, davor und danach. Aber doch einem spezifischen Ausstellungszweck folgt, in dem Fälle von »emotional impact« in einem allen Exponaten gemeinsamen Metier - Klavierquintett einander gegenübergestellt werden. In Neugierde darauf, was in dieser Verbindung über den jeweiligen einzelnen Ausstellungsgegenstand hinaus sichtbar wird beim Flanieren durch diese imaginierte, virtuelle Ausstellung. Und was ich selbst über mich und die Sache lerne in dieser Selbstbeobachtung meines eigenen Kuratierens.

Für mich jedenfalls ist »emotional impact« eine relevante Qualität von Musik. Die eine solch imaginierte, virtuelle Ausstellung als Anlass, Thema und Auswahlkriterium tragen kann. Die als derart motivierte Ausstellung wiederum einen alternativen Zugang zu Musikgeschichte vorführt. Dabei keineswegs ein künstliches Setting schafft. Sondern einen ganz und gar alltäglichen Zugang zu ihr aufgreift, wie Joachim Jacob und Wolfgang Braungart erläutern, wenn sie in analoger Weise über »schöne Stellen« in Literatur schreiben: 
»Stellenweise trifft uns die Literatur, suchen wir aus, naiv oder versiert, was uns gefällt; so lange, wie Aufmerksamkeit und Konzentration anhalten. Wenn wir uns an Literatur erinnern, erinnern wir uns an Stellen; wenn wir uns über sie unterhalten, machen wir uns auf schöne Stellen aufmerksam. Stellen legen wir genau aus. Darum muss sich auch eine literarische Ästhetik für Stellen interessieren, stärker und vielleicht auch unvoreingenommener, als sie es bislang getan hat. [...] In der schönen Stelle schließlich findet sich alles aufs Engste beisammen. [...] Die schöne Stelle vereint demnach das Finden wie das SichÜberlassen. Sie ist viel mehr als nur eine `schöne〈 Stelle. Sie provoziert die Frage nach dem Gelungenen, das sie einlöst und zugleich begrenzt, und nach dem Verhältnis des Einzelnen zum Ganzen. Die schöne Stelle schließlich zielt auch auf den Augenblick des Glücks in der Lektüre, der, wie im Leben, nicht dauern kann. Darum kann man aber doch von ihm sprechen. «238 


\section{Exkurs II:}

\section{Möglichkeiten und Strategien des Sprechens über Musik}

\section{Problemlage}

Das Sprechen über Musik, die Auseinandersetzung damit, wo wann welche Musik »emotional impact « für einen bereithält, also das sich mit der Sache selbst abgeben in Riethmüllers Sinne, ist freilich gar nicht so leicht. Erst recht das Sprechen über die Wirkung von Musik auf einen selbst und die Rückbeziehung dieses »emotional impact« auf das Gehörte.

Letzteres zu tun ist besonders heikel. Das also, was hier versucht wird. D.h. nachzuspüren, ob man den »emotional impact« irgendwo in der Musik konkret dingfest machen kann. Denn an dieser Stelle gilt es sauber zu trennen: Bei dem, was mich hier interessiert, geht es nämlich nicht um eine Beschreibung von etwas, das als solches objektiv Teil einer Musik ist. Wie ein Metrum oder ein Intervallverhältnis, eine Form oder eine Besetzung. Einzig wird hier mit Martin Seel in Anspruch genommen, dass nicht lediglich etwas als Projektion an die Musik herangetragen wird, sondern eine Reaktion auf etwas in der Musik den Ausgangspunkt für den jeweiligen »emotional impact« bildet. ${ }^{239}$ Nur deswegen lohnt es sich überhaupt, die Rückbeziehung dieses »emotional impact« auf das Gehörte zu versuchen. Das freilich - und darauf sei hier nur kurz hingewiesen - ist eben etwas anderes als auf eine der Musik eingeschriebene Emotion zu verweisen oder deren Widerspiegelung in den eigenen Emotionen in den Fokus zu rücken (Affektenlehre) oder gar zum Maßstab zu machen. ${ }^{240}$ Ganz wie Jerrold Levinson betont: 
»Note also, as many writers on this subject have affirmed, that perceiving emotional expressiveness in music does not require feeling the corresponding emotion, or any emotion at all, while listening. $\ll^{241}$

Der Unterschied hat Folgen. Es gibt z.B. keine Kausalität zwischen Musik und Hörer, die etwa derart ausgestaltet ist, dass wenn ich eine Emotion, die der Musik eingeschrieben ist, nicht fühle, ich die Musik quasi falsch oder defizitär höre und umgekehrt - wie Levinson an anderer Stelle ergänzt. ${ }^{242}$

Die jüngeren Arbeiten von Peter Rinderle, Stefan Zwinggi und Alexander Wilfing zur >musikalischen Expressivität , die Beiträge zu Musik und Emotion in den aktuellen Handbüchern von Patrik N. Juslin und John A. Sloboda sowie Susan Hallam, Ian Cross und Michael Thaut bieten einen guten Einstieg in diesen komplexen und weitverzweigten Themenbereich des Verhältnisses von Musik und Emotion. ${ }^{243}$ Für einen ersten Eindruck eignet Juslins Diskussion von sieben Kernproblemen dieses Forschungsbereichs. ${ }^{244}$ Versehen mit umfassenden Weiterverweisen in die kaum mehr überschaubare, von Musikwissenschaft über Philosophie und Soziologie bis Neurobiologie viele Disziplinen überspannende Literatur. Wie eng diese disziplinären Diskurse mittlerweile miteinander verbunden sind, zeigt stellvertretend der jüngste Streit zwischen den Philosophen Peter Kivy und Noël Carroll zu Emotion, Stimmung und Musik unter Rückgriff auf die naturwissenschaftlichen Debatten. ${ }^{245}$

Mir freilich genügt an dieser Stelle ein kleiner Ausschnitt aus diesem weiten Feld als Aufgabe, in meinem Bestreben, den »emotional impact« auf mich beim Hören bestimmter musikalischer Situationen über bewusstes Hören in der Musik konkret zu verorten und hinterher diesen Ort mit den Mitteln der Sprache zu zeigen. Alles andere sind Folge- und Parallelfragen, die hier außen vor bleiben können.

Das Problem des Sprechens über Musik wird hierdurch freilich nicht kleiner. Im Gegenteil. Das sich mit der Sache selbst abgeben in Riethmüllers Sinne - oder besser: das auf die Sache selbst zurückgeworfen sein tritt als Problem noch stärker in den Vordergrund. Die Herausforderung ist an dieser Stelle groß, will man planen, wie ein zielgerichtetes Flanieren durch die besagte imaginierte, virtuelle Ausstellung aussehen, ja überhaupt erst ermöglicht werden könnte. Nicht wenige streiten bekanntlich zur Gänze ab, dass dies überhaupt ginge - über Musik zu sprechen -, obwohl sie an der Möglichkeit eines solchen Sprechens für andere Künste überhaupt keine 
Zweifel hegen, wie Susan McClary berichtet. ${ }^{246}$ Und ziehen sich zu Verteidigung und Beleg auf Stereotypen zurück wie dem viel zitierten Satz >Über Musik zu schreiben sei wie über Architektur zu tanzen $\varsigma^{247}$ - eine Sichtweise, die Lawrence Kramer eine offensichtliche kulturelle Fiktion und Robert Christgau nicht ohne Grund schlicht ignorant nannte, letzteres unter Hinweis auf die Rolle von Raum in Tanz wie Architektur. ${ }^{248}$ Gedankenfaul wäre vielleicht noch treffender. Denn was nützt es, abzustreiten, dass es geht, um es dann allenthalben doch zu tun?

Denn all dies ist natürlich eine seltsame Situation. Man bedenke nur, in wie vielen Konstellationen das Sprechen über Musik Kernaufgabe einer etablierten Berufstätigkeit ist. Vom Musikpädagogen über den Musikwissenschaftler bis zum Musikjournalisten und Musikvermittler. Man vielmehr mit Albrecht Riethmüller sagen muss, dass die »Flut der (sprachlichen) Information, die wir über Musik erhalten, [...] heute tatsächlich massenhaft geworden $\ll$ ist. ${ }^{249}$ Und das Kommunikation zwischen Musikern ausschließlich nonverbal und allenfalls unterstützt von Notationen jedweder Art ablaufe, taugt nicht einmal zur Legende. Jede Probe ist Beleg für das Gegenteil. Abgesehen davon, dass Partituren meist voll sind an Worten und im Übrigen diskursive Sprache schlicht nicht ersetzen können, wie Nelson Goodman ausführlich erläutert hat. ${ }^{250}$

Nichtsdestotrotz ist das Problem da. Ein Problem, über das Einigkeit besteht. Und dem auch ich in meiner sonstigen wissenschaftlichen Arbeit sonst oft ausweiche. Auch ausweichen kann, oft will und bisweilen gar muss, etwa wenn wie im ersten Kapitel ganz andere Gesichtspunkte interessieren. Aber doch ist es für mein Anliegen hier ein vorab adressierungsbedürftiges Problem. Schon, um der imaginierten, virtuellen Ausstellung eine gewisse Kohärenz verleihen zu können. Was kann man eigentlich über Musik sagen? Allzumal, wenn einen keine technische oder statistische Frage, sondern etwas vermeintlich vages wie ihr »emotional impact« interessiert? Und wie? Will man etwas im Wittgensteinschen Sinne zeigen, möchte man, dass es zumindest dem Grunde nach die Chance erhält, nachvollzogen werden zu können. Man muss daher zumindest eine Annäherung an »den Zustand des Sprechens über Musik und damit [an] die Interaktion von Vorstellung und Urteil, Begriff und Sache« versuchen, wie es bei Riethmüller heißt. ${ }^{251}$ Sich seiner Optionen versichern.

Sprechen über Musik ist ein altes Problem. Letztlich ungelöst, seitdem wir Aufzeichnungen über Musik haben. Also seit gut zweieinhalbtausend 
Jahren. Obwohl unaufhörlich über Musik kommuniziert wird, ist uns, wie Riethmüller unter Rekurs auf Hans Heinrich Eggebrecht diagnostiziert, »an der Musik - und das ist nicht zu bestreiten - das Sprechen über Musik das Fremdeste von allem « ${ }^{252}$ Freilich entmutigt einen das Ausmaß der Herausforderung nur bis zu dem Augenblick, in dem man die Erfahrung macht, dass Sprechen über Musik funktionieren kann, wie Robert DiYanni so treffend resümiert:

»Good music writing, nonetheless, somehow succeeds in making sense; it makes sense in what it says directly about music, and in what it suggests indirectly, in what circumscribes the music as well. $\ll^{253}$

Die Frage ist halt nur, wenn man den Kommunikationserfolg nicht völlig dem Zufall oder der Intuition überlassen will, ob man irgendwo systematische Hilfe bekommen kann hinsichtlich der sprachlichen Mittel, die sich zur Nutzung anbieten?

Ein Blick in die verfügbare Literatur zum Thema enttäuscht allerdings rasch. Sucht man nämlich nach Orientierung, findet man überraschend wenig an systematischen Angeboten für dieses spezielle Anliegen. Dafür muss man zum Beleg nur in musikologische Standardlehrbücher schauen. Hier, wo man dies vielleicht zu allererst zu finden erwarten würde, stehen stattdessen Fragen des wissenschaftlichen Arbeitens und Recherchierens sowie der Methodologie im Vordergrund. ${ }^{254}$ Auch Einführungen, die >Schreiben über Musikı als Titel führen, konzentrieren sich weithin auf Rahmenfragen wie die Gewinnung von belastbaren Informationen und beschäftigen sich ansonsten mit der Einführung in Standardformate wie Essay und Rezension und diesbezügliche allgemeine stilistische Hinweise. ${ }^{255}$ Selbst eine so fulminante Zurückweisung des Topos von der Unbeschreibbarkeit der Musik wie Lawrence Kramers Interpreting Music und The Thought of Music enthalten nur Hinweise hier und da, wie man das denn tun könne. ${ }^{256}$ Nicht anders als die gar nicht so geringe Literatur zu typischen Professionen des Sprechens über Musik, z.B. dem Arbeitsbereich der akademischen und journalistischen Musikkritik. Auch diese steigt oft nicht in die konkreten Möglichkeiten und Konventionen der sprachlichen Auseinandersetzung mit Musik ein, sondern konzentriert sich auf andere Aspekte wie die soziale, ökonomische und technische Entwicklung des Berufsfelds. ${ }^{257}$ Insofern vermeidet man den Versuch einer systematischen 
Annäherung unter Verweis darauf, dass dies dem Feld fremd wäre, wie es z.B. Susan Holtfreter vertritt, aber auch in Schriften wie jenen von Robert D. Schick sowie Marc Woodworth und Ally-Jane Grossan zum Ausdruck gebracht wird:

»Insgesamt entstand jedoch der Eindruck, dass prototypische Strukturtypen für die inhaltliche Ebene der Musikkritik kaum von Relevanz sind. Wenn, dann sind diese in keiner Weise vergleichbar mit der Verbindlichkeit der thematischen Struktur der Nachricht. Vielmehr kann davon ausgegangen werden, dass etablierte Strukturen nahezu variiert und aufgebrochen werden müssen. Die bisher mehrfach festgestellte kreative Selektion der sprachlichen Mittel trifft auch auf die inhaltliche Struktur der Musikkritik zu. Insofern fordert die Musikkritik hohe Schreibkompetenz, da kaum auf verbindliche Strukturmuster zurückgegriffen werden kann, sondern diese für jeden Text aus dem Gegenstand neu abgeleitet werden. $\ll^{258}$

Punktuell findet man natürlich rasch Vertiefungen. Etablierte Klassiker, die einem in Teilaspekten dabei weiterhelfen, Grund unter den Füßen zu gewinnen, um genaues sagen zu können. Etwa zu formalen Standards des Sprechens über Musik wie D. Kern Holomans Writing about Music oder zur musikalischen Begriffsgeschichte wie das Handwörterbuch der musikalischen Terminologie. ${ }^{259}$ Überhaupt werden gerade technische Begriffe natürlich in Legionen von Lehrbüchern vermittelt, die seit jeher und ungebrochen zu allen möglichen Bereichen des musikalischen Handwerks verfasst werden, von der Instrumentalpraxis über die Produktionstechnik bis zur Musiktheorie.

Aber all dies bleibt Teilaspekt. Der Überblick über die Optionen fehlt. Doch der ist wichtig. Denn es gibt ganz unterschiedliche Motivationen und soziale Anlässe, über Musik sprechen zu wollen, mit ganz verschiedenen Vermittlungszielen und mit einem korrespondierend heterogenen Feld an potentiellen Adressaten, ihren Interessen an Musik und dem bei ihnen voraussetzbaren Wissen über Musik. ${ }^{260}$ Entsprechend ausdifferenzierte, bisweilen gar entgegengesetzte Traditionen existieren, wie das sich mit der Sache selbst abgeben in Riethmüllers Sinne darstellungsseitig zu geschehen habe. ${ }^{261}$ Man denke nur an den geradezu dogmatischen Verzicht auf technisches Sprechen über Musik in journalistischen Kontexten. Oder umgekehrt 
die Abneigung gegen Metaphern im Kontext musiktheoretischer Analyse. Man hat also ganz offensichtlich Optionen zur Hand. Nur eben welche?

Die Suche nach Orientierung wird auch nicht dadurch erleichtert, dass sich unter dem Lemma Musik und Sprache ein großer Teil der Literatur zunächst einmal überhaupt gar nicht mit dem Sprechen über Musik beschäftigt, sondern mit der Frage, inwiefern eine Annäherung an Musik in Analogie zur Sprache gefunden bzw. Musik überhaupt als Sprache verstanden werden kann. ${ }^{262}$ Man denke nur an die überkommene metaphorische Rede von der >Tonsprache $<$ und der $>$ Klangrede $<.{ }^{263}$ Oder an die hartnäckigen Topoi von `Musik als Universalsprache und $\gg$ Musik als Sprache der Gefühle $<.{ }^{264} »$ Dass Musik eigentlich das Unsagbare verwalte «, wie Diedrich Diederichsen am Ausgangspunkt einer längeren Dekonstruktion schreibt. ${ }^{265}$

Der hingegen ganz konkrete Bereich der tatsächlichen >Tonsprachen in der Sprachwissenschaft und der Ethnologie stellt wieder ein ganz anderes Forschungsfeld dar, auf das man aber gleichfalls unter dem Lemma Musik und Sprache stößt. Ebenso wie jenes der Sprachförderung durch Musik. Des Weiteren sind unter dieser Oberkategorie zahlreiche Studien zu Prosa und Lyrik über Musik versammelt. Mit Fokus z.B. auf der Verwendung musikalischer Mittel, Formen und Strategien in Literatur - und umgekehrt. Und natürlich verorten sich an dieser Stelle endlos viele Schriften über Vokalmusik im Allgemeinen und Vertonungen im Besonderen mit Fokus auf das Verhältnis von Musik und Sprache hierbei.

All das sind nur Beispiele für Themenbereiche unter dem Stichwort Musik und Sprache. Dabei allesamt weithin ausdifferenzierte, zum Teil recht alte Forschungsfelder. Musik und Sprache ist ein weit verzweigtes Gebiet. Und meine Aufgabe hier, vorab eine systematische Annäherung an meine Optionen zu gewinnen, mit den Mitteln der Sprache »emotional impact « in ausgewählten musikalischen Situationen in Klavierquintetten verorten zu können, sprich mich meines Handwerkzeugs zu versichern, ist nur ein kleiner Bereich hierin. Obendrein ein vergleichsweise wenig erkundeter. So wie umgekehrt die Kommunikation über Musik mit den Mitteln der Sprache nur ein Teilgebiet der Musikvermittlung darstellt, die vom Zeigen durch Vorspielen über multimediale Erläuterungsansätze bis zu partizipativen Mitmachstrategien reicht.

Aber die greifbaren Orientierungsangebote vor allem aus Sprachwissenschaft, Pädagogik und der Analyse des Arbeitsfelds Musikkritik erlauben in Verbindung mit der eigenen Untersuchung von Praxisbeispielen, die 
einem besonders gelungen und ertragreich erscheinen, doch wenigstens eine erste Annäherung an eine Systematik. Was einem die Möglichkeit eröffnet, sich der anschließenden Aufgabe doch von einem gewissen Reflexionsniveau aus zuzuwenden. Zielgerichtet also. Mit einer wenigstens gewissen Sicherheit hinsichtlich der einem zur Verfügung stehenden Optionen. Ihrer Stärken und Schwächen.

Warum all die Mühe? Manchmal finden Ausstellungsgegenstände ihren Weg in solche imaginierten, virtuellen Ausstellungen natürlich auch bei mir auf anderem Wege. Durch Zufall etwa. Oder über das von der Faszination an der Sache selbst weithin verselbstständigte historische Metanarrativ des Kanons. Oder über die Mathematik automatisierter Algorithmen, etwa Playlists auf Spotify oder Ähnlichkeitsangebote auf YouTube. Und ab und zu sogar über die anonymisierende Großdatenpolitik der digitalen Geisteswissenschaften, wenn hierdurch in Datenbaken plötzlich etwas auftaucht, von dem man noch nie gehört und das den Anfang einer Spurensuche bildet, an dessen Ende etwas mit »emotional impact« auf mich steht. Sprechen über Musik bringt an dieser Stelle aber mit Abstand den größten Ertrag. Für mich am häufigsten. Etwas mit Hilfe von Worten in Musik konkret gezeigt zu bekommen, als Text, im Gespräch. Solch Wittgensteinsches Zeigen kann freilich bei mir mit ganz unterschiedlichen Sprachstrategien zum Erfolg führen, so zeigt die Erfahrung. Von technischem Sprechen bis hin zu metaphorischem, von musikalischen Vergleichen bis zu außermusikalischen Kontextualisierungen. Jedenfalls für mich, an dieser Stelle, hat sich keine überlegene Sprechstrategie durchgesetzt. Es gibt eine Balance. Eine Gleichwertigkeit. Und oft greift sogar erst eine Kombination der spezifischen Stärken der verschiedenen verbalen Zeigestrategien. Technisches Sprechen und Zeigen allein jedenfalls, das, was ich als Musikwissenschaftler gelernt habe, reicht nicht. Jedenfalls nicht für mich. Nicht für sich allein. Nicht an dieser Stelle. Wenn es um etwas wie »emotional impact« geht. Egal, ob ich typische Darstellungsweisen der Musikanalyse zu Rate ziehe oder statistische Auswertungen oder Tabellen mit Messdaten. Da ist meine Selbstbeobachtung ganz bei Lawrence Kramers Analyse:

»Either way, musical meaning forfeits in advance any possible claim to represent musical knowledge. Music appears in this scenario as an intrinsically selfmystifying phenomenon. The more it incites subjectivity - that is, does just what it is supposed to do - the less responsive it becomes to description. The features 
we can describe, form and technique, cannot account adequately - if at all - for music's subjective effects. ${ }^{266}$

Nicht ohne Bedauern bin ich hier ganz bei Kramer. Denn wäre es anders, würde technisches Sprechen und Zeigen reichen, ich könnte das Problem über den Erwerb und das Einüben von fachspezifischem Wissen und wissenschaftlichen Beschreibungsstandards lösen. Was bei anderen Aufgabenstellungen in meiner akademischen Arbeit auch so funktioniert. Nur hier eben nicht.

Für meine Tätigkeiten, in der Lehre wie im Schreiben über Musik, habe ich jedenfalls vor dem Hintergrund dieser Selbstbeobachtung eine Systematik erstellt, mit der ich arbeite. Die keineswegs für sich beansprucht, abschließend zu sein. Im Gegenteil ein work in progress darstellt. Aber mir doch eine erste strukturierte Annäherung an die Aufgabe erlaubt, über Musik zu sprechen. In ihr etwas Ästhetisches wie z.B. »emotional impact« zu zeigen. Mir Orientierung gibt. Mich meiner Optionen versichert. Und erlaubt, Vertiefungen zu Teilaspekten wie technischen Begriffen oder metaphorischen Strategien gezielt darin aufzuhängen und weiterzuverfolgen.

Diese Systematik mag vielleicht auch anderen nützen. Im Suchen nach etwas, dass Lawrence Kramer constructive descriptions nennt, »a statement, often only a sentence or two, in which interpretive energy is concentrated. $\aleph^{267}$ Ich zumindest habe nach einer solchen gesucht und keine gefunden. Jedenfalls ist sie Basis der nachfolgenden Ausstellungsbeschreibungen, des dortigen Herantastens an den »emotional impact« ausgewählter musikalischer Situationen in Klavierquintetten. So mag dieser hier nur in aller Kürze durchgeführte Exkurs in die besagte Systematik zumindest helfen, das je nachdem Gelingen oder Scheitern der nachfolgenden Situationsbeschreibungen nachzuvollziehen.

In der Lehre verwende ich zur Erläuterung der Systematik wie ihrer Bestandteile regelmäßig einen journalistischen Text. Einen Nachruf auf David Bowie, der unter dem Titel Der Unfassbare am 12. Januar 2016 im Berliner Tagesspiegel erschienen ist, von Kai Müller stammt und frei im Internet abrufbar ist. ${ }^{268}$ Dieser Text ist für diesen Zweck besonders gut geeignet, da der Autor Bowies charakteristische Vielfalt darin spiegelt, dass er enorm viele verschiedene sprachliche Annäherungsstrategien an Bowies Musik zumindest andeutet. Bisweilen aber auch extensiv benutzt und kombiniert, was hier als Schreibtechnik außen vor bleiben kann, dem Text aber zusätz- 
lichen Mehrwert als Fallbeispiel für die Lehre im Bereich Musikvermittlung verleiht. Über kurze Auszüge weise ich in den Endnoten zum Beleg und zur Illustration jeden Teilpunkts der Systematik jeweils auf Beispiele aus Müllers Text hin, sofern dort vorhanden.

Die Systematik gliedert sich in vier Bereiche (vgl. Tab. 1):

- Annäherung über Vergleich, Zuordnung und Einflussanalyse

- Annäherung über die Beschreibung der Machart

- Annäherung über die Beschreibung des Ausdrucks

- Annäherung über die Beschreibung des Kontexts

\section{VIER MOdi DER ANNÄHERUNG}

\section{Annäherung über Vergleich, Zuordnung und Einflussanalyse}

Größte Herausforderung in der Kategorie Annäherung über Vergleich, Zuordnung und Einflussanalyse ist, dass alle Annäherungsstrategien dieser Rubrik Wissen auf Seiten des Adressaten voraussetzen. Je mehr Wissen vorausgesetzt werden kann, desto präziser kann ich auf diesem Wege etwas über Musik sagen. Je weniger vorausgesetzt werden kann, desto vager bleibt die Vergleichsebene. Je vager jedoch die Vergleichsebene konturiert ist, desto breiter sind die Auslegungsmöglichkeiten auf Seiten des Adressaten und damit das Risiko auf Seiten des Kommunizierenden, dass nicht oder nicht vollständig ankommt, was er vermitteln will:

GENREBEGRIFFE: Annäherung an die ästhetischen Qualitäten einer Musik mittels Genreeinordnung/-zuordnung. ${ }^{269}$

MusikeRVERGLEICH: Annäherung an die ästhetischen Qualitäten einer Musik durch ins Verhältnis setzen dieser Musik zur Musik anderer Musiker, um hierüber Gemeinsamkeiten oder Unterschiede zu benennen. ${ }^{270}$

WERKVERGLEICH: Annäherung an die ästhetischen Qualitäten einer Musik durch ins Verhältnis setzen dieser Musik zu (eigenen wie fremden) anderen Werken, um hierüber Gemeinsamkeiten oder Unterschiede zu benennen. $^{271}$ 


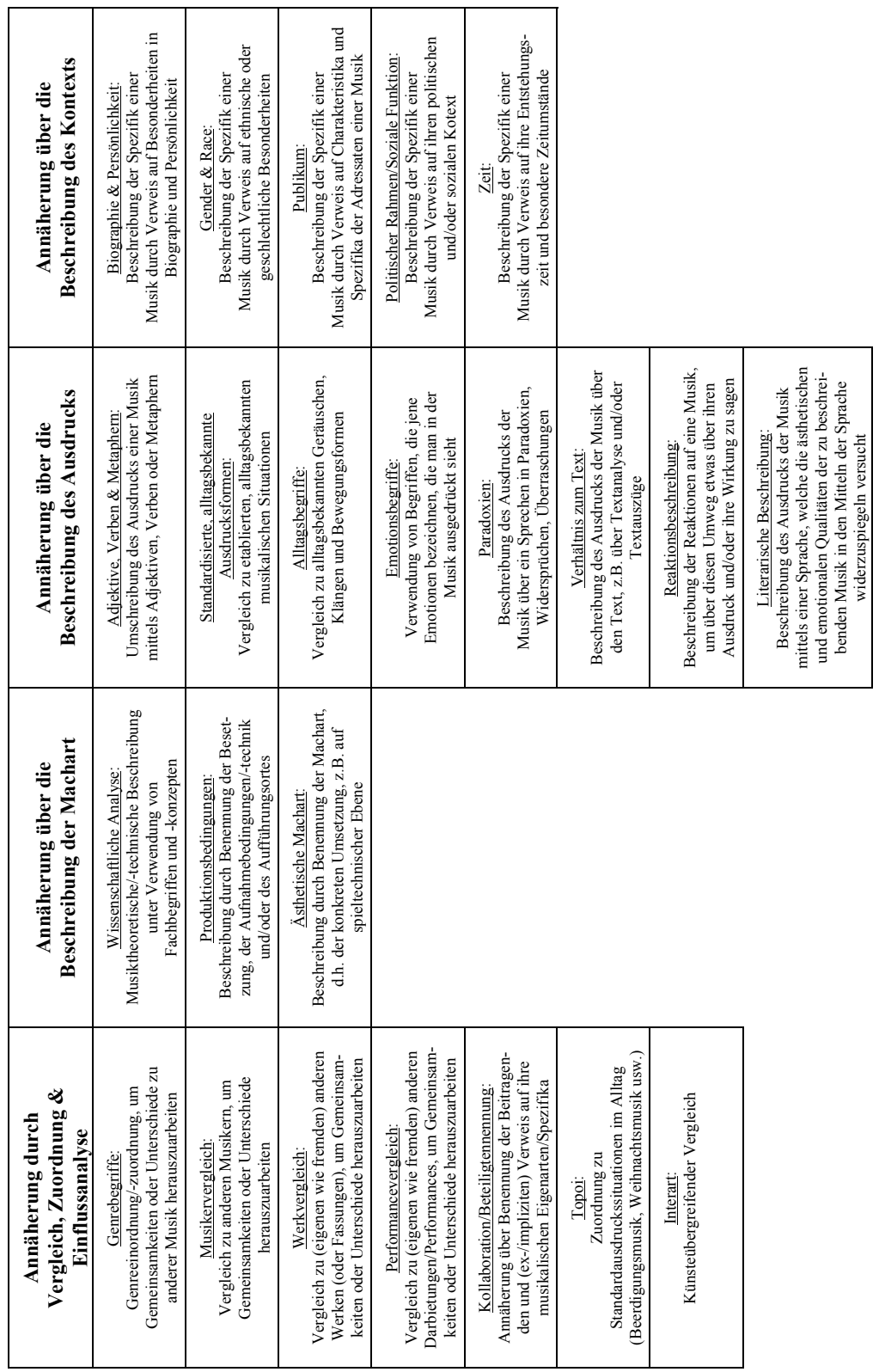

Tab. 1: Strategien des Sprechens über Musik: Vier Modi der Annäherung. 
PerformanceVergleich: Annäherung an die ästhetischen Qualitäten einer Musik durch ins Verhältnis setzen der Darbietungsweise dieser Musik zu (eigenen wie fremden) anderen Darbietungen/Performances, um hierüber Gemeinsamkeiten oder Unterschiede zu benennen. ${ }^{272}$

Kollaboration/Beteiligtennennung: Annäherung an die ästhetischen Qualitäten einer Musik über Benennung der Beitragenden, deren künstlerische Eigenarten/Spezifika beschrieben werden oder das Wissen darüber als bekannt vorausgesetzt wird. ${ }^{273}$

TOPOI: Annäherung an die ästhetischen Qualitäten einer Musik durch ins Verhältnis setzen zu Standardausdruckssituationen im Alltag (Beerdigungsmusik, Weihnachtsmusik, Hochzeitsmusik usw.). ${ }^{274}$

INTERART: Annäherung an die ästhetischen Qualitäten einer Musik durch künsteübergreifenden Vergleich. ${ }^{275}$

\section{Annäherung über die Beschreibung der Machart}

Größte Herausforderung in der Kategorie Annäherung über die Beschreibung der Machart ist ebenfalls, dass alle Annäherungsstrategien dieser Rubrik Wissen auf Seiten des Adressaten voraussetzen. Hier geht es um ein Wissen, das anders als die z.B. im Musikjournalismus noch häufig genutzte erste Kategorie Annäherung über Vergleich, Zuordnung und Einflussanalyse diesmal ausdrücklich musikalische Fachleute erwartet. `Nerd talkı. Man findet diese Annäherungsweisen daher besonders oft in der Kommunikation von musikalischen Fachleuten untereinander:

WISSENSCHAFTLICHE ANALYSE: Annäherung an die ästhetischen Qualitäten einer Musik über eine musiktheoretische/-technische Beschreibung unter Verwendung von Fachbegriffen und -konzepten. ${ }^{276}$

Produktionsbedingungen: Annäherung an die ästhetischen Qualitäten einer Musik über die Benennung der Besetzung, der Aufnahmebedingungen/-technik und/oder des Aufführungsrahmens, z.B. der Akustik des Aufführungsortes. ${ }^{277}$

ÄSTHETISCHE MACHART: Annäherung an die ästhetischen Qualitäten einer Musik über durch Beschreibung ihrer Machart, d.h. ihrer konkreten Umsetzung durch die Musiker, z.B. auf spieltechnischer Ebene. ${ }^{278}$ 


\section{Annäherung über die Beschreibung des Ausdrucks}

Die dritte Kategorie Annäherung über die Beschreibung des Ausdrucks ist qua ihrer Beschaffenheit vermeintlich am nächsten an dem, was ich hier als »emotional impact« verfolge. Und gewiss die gerade im alltäglichen Sprechen über derlei ästhetisches Erleben die am häufigsten gebrauchten Annäherungsweise, insbesondere unter Nichtfachleuten. Auch sind die literarischen Möglichkeiten in dieser Kategorie am größten, die Nähe zur Literatur am stärksten ausgeprägt.

Paradoxerweise ist es aber zugleich die unsicherste, instabilste Annäherungsweise, wie Frank Sibley in seinem grundlegenden Essay Aesthetic Concepts erläutert hat, da derartige ästhetische Termini begrifflich nie klar bestimmt sind und zum Zwecke der Beschreibung daher nicht unstrittig und verlässlich verwendet werden können. Die Vertiefung von Fachwissen hilft daher hier anders als im Fall der ersten beiden Kategorien also nur bedingt weiter und kann einem lediglich zu einem empirisch abgesicherten Gespür für Konventionen verhelfen:

»[...], ich möchte in diesem Aufsatz zeigen, daß es keine nicht-ästhetischen Merkmale gibt, die unter irgendwelchen Umständen als logisch hinreichende Bedingungen für die Anwendung ästhetischer Termini fungieren. Ästhetische Begriffe oder Geschmacksbegriffe sind in dieser Hinsicht keinerlei Bedingungen unterworfen.

Man ist kaum versucht anzunehmen, ästhetische Termini ähnelten Wörtern wie >quadratisch`, die gemäß einer Anzahl von notwendigen und hinreichenden Bedingungen angewandt werden. Denn während ein Quadrat stets auf Grund derselben Bedingungen ein Quadrat ist (vier gleiche Seiten und vier rechte Winkel), haben ästhetische Termini für ganz verschiedene Objekte Gültigkeit. Ein Ding ist anmutig, ein anderes wegen jener Merkmale, und so fast endlos weiter. [...]

Es gibt keine hinreichenden Bedingungen, keine nicht-ästhetischen Merkmale derart, daß das Vorhandensein einer Reihe oder einer gewissen Anzahl von ihnen die Anwendung eines ästhetischen Terminus logisch rechtfertigen und auBer jeden Zweifel setzen könnte. «279 $^{27}$

Dieser Vorbehalt ist jedoch kein grundsätzlicher Einwand gegen die nachfolgend aufgeführten Annäherungsweisen. Denn wie Sibley im selben 
Atemzug erläutert, können wir nicht auf sie verzichten und uns etwa auf die ersten beiden Kategorien zurückziehen und uns schlicht auf die bessere Ausbildung unserer Adressaten konzentrieren, wenn uns jedenfalls an ästhetischen Dimensionen der Sache wie ihrem »emotional impact« gelegen ist:

»Die Dinge können uns mit nicht-ästhetischen Termini ausführlich beschrieben werden wie wir wollen, wir werden dadurch nicht in die Lage versetzt, zugeben zu müssen oder nicht leugnen zu können, daß die Dinge zart, anmutig, grell oder ungewöhnlich ausgewogen sind. « $^{280}$

AdJeKtive, Verben \& MEtaPHERN: Annäherung an die ästhetischen Qualitäten einer Musik durch Umschreibung ihres Ausdrucks mittels Adjektiven, Verben oder Metaphern. ${ }^{281}$

StAndardisierte, AlltagsbeKanNTE AusdrucKSfORmEn: Annäherung an die ästhetischen Qualitäten einer Musik durch Vergleich zu etablierten, alltagsbekannten musikalischen Ausdrucksformen. ${ }^{282}$

AlltaGSBEGRIFFE: Annäherung an die ästhetischen Qualitäten einer Musik durch Vergleich zu alltagsbekannten Geräuschen, Klängen und Bewegungsformen. ${ }^{283}$

EMOTIONSBEGRIFFE: Annäherung an die ästhetischen Qualitäten einer Musik durch Verwendung von Begriffen, die jene Emotionen bezeichnen, die man in der Musik ausgedrückt sieht. ${ }^{284}$

PARADOXIEN: Annäherung an die ästhetischen Qualitäten einer Musik durch Beschreibung des Ausdrucks der Musik über ein Sprechen in Paradoxien, Widersprüchen, Überraschungen. ${ }^{285}$

VERHÄLTNIS ZUM TEXT: Annäherung an die ästhetischen Qualitäten einer Musik über den Text, z.B. durch Textanalyse und/oder Textauszüge. ${ }^{286}$

REAKTIONSBESCHREIBUNG: Annäherung an die ästhetischen Qualitäten einer Musik durch Beschreibung der Reaktionen auf eine Musik, um über diesen Umweg etwas über ihren Ausdruck und/oder ihre Wirkung zu sagen. $^{287}$

LITERARISCHE BESCHREIBUNG: Annäherung an die ästhetischen Qualitäten einer Musik mittels einer Sprache, welche die ästhetischen und emotionalen Qualitäten der zu beschreibenden Musik in den Mitteln der Sprache widerzuspiegeln versucht, etwas zum Ausdruck der Musik sprachlich Analoges zu schaffen versucht. ${ }^{288}$ Gerade im Musikjournalismus wird an dieser 
Stelle viel über den persönlichen Stil - den 〉Sound ` oder 〉Sprachrhythmus - eines Autors in seinem Sprechen über Musik reflektiert und räsoniert, ebenso wie über die Leidenschaft für die Sache, die er zu übertragen in der Lage ist. ${ }^{289}$ Im Akademischen ist vielleicht Jacques Derrida der prominenteste Vertreter dieser Strategie, durch die Art des Schreibens über etwas beim Leser Erkenntnis auszulösen, nicht dadurch, dass er direkt anspricht, was es zu Erkennen gilt (>différance`), wie z.B. sein Text Das Gesetz der Gattung in exemplarischer Weise vorführt. ${ }^{290}$

\section{Annäherung über die Beschreibung des Kontexts}

Die Annäherung über die Beschreibung des Kontexts scheint mir die vagste Option zu sein. Gerade in politisch sensiblen Bereichen stößt man aber immer wieder auf sie. Oft mit Vehemenz. Etwa bei der Reklamation von Hautfarbe und ethnischer Zugehörigkeit als wesentlich in der Historiographie von Jazz oder Hip-Hop. Oder von Geschlechtsspezifik in Genderkontexten, gerade wenn es um Stimmen/Gesang geht. Daneben erscheinen solche Äußerungen auch in traditionell gestimmten Kontexten, die Musik vor allem autobiographisch deuten, etwa hinter Stellvertreterbegriffen wie Authentizität. Und schließlich kommen solche Umschreibungen häufig in Vermittlungskontexten vor, in denen davon ausgegangen wird, dass man beim Adressaten keinerlei Fachwissen voraussetzen kann.

Hier seien an möglichen Kategorien stellvertretend nur drei Beispiele für kontextuelle Annäherungen genannt, so wie sie eben in Müllers BowieText erscheinen. Die Annäherung über die Beschreibung des Kontexts ist jedoch die potentiell offenste Kategorie mit dem in der Praxis größten Reichtum an Varianten. ${ }^{291}$ Korrespondierend zu ihrer Vagheit. Andere typische Annäherungsweisen an dieser Stelle (vgl. Tab. 1) konzentrieren sich z.B. auf die politischen Bedingungen, unter denen eine Musik entsteht, oder ihre soziale Funktion. Oder rekurrieren auf die Entstehungszeit einer Musik und besondere Zeitumstände. ${ }^{292}$ Zentral an dieser Stelle ist nur, dass mit den jeweiligen kontextbezogenen Aussagen die Absicht verbunden ist, zumindest auch etwas über das zu Hörende selbst zu sagen:

BIOGRAPHIE \& PERSÖNLICHKEIT: Annäherung an die ästhetischen Qualitäten einer Musik durch Verweis auf Charakteristika und Spezifika in Biographie und Persönlichkeit des zu Hörenden. ${ }^{293}$ 
GENDER \& RACE: Annäherung an die ästhetischen Qualitäten einer Musik durch Verweis auf ethnische oder geschlechtliche Besonderheiten des zu Hörenden. ${ }^{294}$

PUBLIKUM: Annäherung an die ästhetischen Qualitäten einer Musik durch Verweis auf Charakteristika und Spezifika der Adressaten einer Musik. 295 



\section{Emotional Impact: 67 Situationen seit Schumann}

\section{Die Ausstellung}

BRAHMS, Johannes (1833-1897): Klavierquintett f-Moll op. 34 (1864).

ReferenZaufnahme: 2013, Live beim Festival Wissembourg (Nikita Mndoyants, Quatuor Ebène). ${ }^{296}$ NoTEN: Leipzig: Breitkopf \& Härtel 1926 (via IMSLP). ${ }^{297}$ SituAtion: II. Satz: Andante, un poco adagio (T. 118-126), ca. 24.02-24.45 $\mathrm{min}$.

Castillon de Saint-Victor, Marie-Alexis de (1838-1873): Klavierquintett Es-Dur op. 1 (1864).

ReferenZaufnahme: 2009, Lidi 0303310-10 (Laurent Martin, Quator Satie). Noten: Paris: Durand o.J. (ca. 1890) (via IMSLP - Erstveröffentlichung Paris: Flaxland 1865). SitUATION: Satz II: Scherzo (T. 278-300), ca. 3.48-4.25 $\min$.

RAFF, Joachim (1822-1882): Klavierquintett a-Moll op. 107 (1864).

Referenzaufnahme: 2003, MDG 304 1181-2 (Ensemble Villa Musica). Noten: Leipzig: Schuberth 1864 (via IMSLP). SituAtion: Satz III: Andante, quasi larghetto mosso (Buchstabe A, T. 7-10), ca. 1.52-2.04 min.

SgambatI, Giovanni (1841-1914): Klavierquintett Nr. 1 f-Moll op. 4 (1866).

ReFEREnZAufnAhme: 1998, ASV CD DCA 1029 (Francesco Caramiello, Ex Novo Quartet). Noten: Mainz: Schott 1877 (via IMSLP). Situation: Satz III: Andante sostenuto (Buchstabe E, T. 19-22), ca. 9.45-10.10 min. 
MARTUCCI, Giuseppe (1856-1909): Klavierquintett C-Dur op. 45 (1878). Referenzaufnahme: 1992, Claves CD 50-9210 (Mario Borciani, Giovane Quartetto Italiano). Noten: Leipzig: Kistner 1893 (via IMSLP). SitUATION: Satz II: Andante con moto (T. 80-91), ca. 3.41-4.09 min.

FRANCK, César (1822-1890): Klavierquintett f-Moll (1879).

ReferenZaufnahme: 2014, Live beim Festival Wissembourg (Vyacheslav Gryaznov, Quatuor Ebène). ${ }^{298}$. NOTEN: Paris: Hamelle 1880 (via IMSLP). SITUATION: Satz I: Molto moderato quasi lento (Buchstabe E, T. 5-8), ca. 4.47$4.54 \mathrm{~min}$.

SINDING, Christian (1856-1941): Klavierquintett e-Moll op. 5 (1885).

ReferenZaufnahme: 1994, Edition Abseits EDA 007-2 (Pihtipudas Kvintetti). Noten: Kopenhagen: Wilhelm Hansen 1888 (via IMSLP). SitUATION: Satz III: Intermezzo (T. 541-560), ca. 5.46-5.59 min.

ZARĘBSKI, Juliusz (1854-1885): Klavierquintett g-Moll op. 34 (1885).

Referenzaufnahme: 2005, DUX 0530 (The Warsaw Quintet). Noten: Warschau: Towarzystwo Wydawnicze Muzyki Polskiej 1931 (via IMSLP). SITUATION: Satz IV: Finale: Presto (T. 320-327), ca. 5.37-5.45 min.

DvořÁK, Antonín (1841-1904): Klavierquintett Nr. 2 A-Dur op. 81 B. 155 (1888).

Referenzaufnahme: 2006, Philips E4757560 (Swjatoslaw Richter, Borodin Quartet - Reissue, Aufnahme von 1982). Noten: Berlin: Simrock 1888 (via IMSLP - zahlreiche aktuelle Notenausgaben erhältlich). SitUATION: Satz III: Scherzo (Furiant) (T. 134-161, Poco tranquillo), ca. 1.27-1.50 min.

SibELIUS, Jean (1865-1957): Klavierquintett g-Moll (1890).

Referenzaufnahme: 1994, EDA 007-2 (Pihtipudas Kvintetti). Noten: Kopenhagen: Wilhelm Hansen 1993. SituAtion: Satz II: Intermezzo, ca. 1.32$1.44 \mathrm{~min}$.

FRÜHLING, Carl (1874-1947): Klavierquintett fis-Moll op. 30 (1892).

REFERENZAUFNAHME: Es ist keine vollständige Aufnahme öffentlich frei zugänglich, aber zumindest Ausschnitte auf der Website Edition Silvertrust. ${ }^{299}$ Noten: Leipzig: Leuckart 1894 (via IMSLP). SituATION: Satz II: Andante cantabile, T. 1-8, ca. 0.00-0.30 min.

SuK, Josef (1874-1935): Klavierquintett g-Moll op. 8 (1893).

Referenzaufnahme: 2004, Hyperion CDA67448 (The Nash Ensemble). Noten: Prag: Hudební Matice 1921 (via IMSLP). SituAtion: Satz I: Allegro energico (Ziffer 8, T. 1-8), ca. 2.53-3.07 min. 
GranAdOS, Enrique (1867-1916): Klavierquintett g-Moll op. 49 (1894). Referenzaufnahme: 2001, Columna Música 1CM0082 (Mac McClure, Quartet Glinka). Noten: Barcelona: Editorial de Música Boileau 2004. SitUATION: Satz III: Largo - Molto Presto, ca. 1.19-2.01 min.

NovÁK, Vitězslav (1870-1949): Klavierquintett a-Moll op. 12 (1897).

Referenzaufnahme: 1994, Centaur CRC 2191 (Jírí Skovasja, The Kubín Quartet). Noten: Berlin: Simrock 1904 (via IMSLP). SituAtion: Satz III: Slovakisch: Allegro risoluto (Buchstabe H, T. 1-8), ca. 2.04-2.18 min.

RYELANDT, Joseph (1870-1965): Klavierquintett a-Moll op. 32 (1901).

Referenzaufnahme: 2008, Phaedra, DDD $92055 »$ In Flanders Fields, Vol. 55« (Jozef de Beenhouwer, Spiegel String Quartet). Noten: Liége: Léopold Muraille o.J. (ca. 1903) (via IMSLP). SituATION: Satz II: Adagio religioso (T. 19-26, 102-110), ca. 1.02-1.1.28, 5.08-5.33 min.

ThuiLle, Ludwig (1861-1907): Klavierquintett Nr. 2 Es-Dur op. 20 (1901). ReferenZaufnahme: (Oliver Triendle, Vogler Quartett). Noten: Leipzig: Kistner 1901 (via IMSLP). SitUATION: Satz I: Allegro con brio (T. 1-10), ca. 0.00-0.16 min.

VAUGHan WiLliams, Ralph (1872-1958): Klavierquintett c-Moll (1903).* ReFerenZaufnahme: 2002, Hyperion CDA673817/2 (The Nash Ensemble). Noten: London: Faber Music 2002 (via IMSLP). SituAtion: Satz I: Allegro con fuoco (Buchstabe Q, T. 8-12 [T. 277-281]), ca. 6.06-6.19 min.

BERGER, Wilhelm (1861-1911): Klavierquintett f-Moll op. 95 (1905).

ReFerenZaufnahme: 1994, MDG 308 0506-2 (Jost Michaels, Verdi-Quartett). Noten: Leipzig: Kahnt 1905 (via IMSLP). SitUAtion: Satz I: Allegro non troppo ed energico (Buchstabe B, T. 8-13), ca. 2.52-3.05 min.

WALTER, Bruno (1876-1962): Klavierquintett fis-Moll (1905).

Referenzaufnahme: 2016, Naxos 8.573352 (Le Liu, Patrick Vida, Lydia Peherstorfer, Sybille Häusle, Stefanie Huber). NOTEN: Wien: Universal Edition 2013. ${ }^{300}$ Situation: II. Satz: Ruhig und heiter, ca. 0.59-1.09 min.

BRIDGE, Frank (1879-1941): Klavierquintett d-Moll H. 49a (1907/1912).

ReFerenzaufnahme: 1996, ASV CD DCA 678 (Allan Schiller, Coull String Quartet). Noten: London: Augener 1919 (via IMSLP). SitUAtion: Satz I: Adagio - Allegro moderato (Ziffer 4 = T. 72-83), ca. 2.47-3.12 min. 
PFITZNER, HANS (1869-1949): Klavierquintett C-Dur op. 23 (1908).

Referenzaufnahme: 1993, Orfeo C 281931 A (Consortium Classicum). Noten: Leipzig: Peters 1909 (via IMSLP). SituAtion: Satz III: Adagio (Buchstabe K, T. 1-16), ca. 10.50-12.03 min.

SCHMITT, Florent (1870-1958): Klavierquintett h-Moll op. 51 (1909).

Referenzaufnahme: 2000, Musidisc 465 810-2 (Werner Bärtschi, Quatuor de Berne - Reissue, Aufnahme von 1981, Ersteinspielung, Erstveröffentlichung 1982, Accord 149 528). Noten: Paris: Mathot 1910 (via IMSLP). Situation: Satz II: Lent (6 T. vor Ziffer 15 bis Ende Ziffer 16), ca. 9.03-10.03 min.

LE FLEM, Paul (1881-1984): Klavierquintett e-Moll (1910).

Referenzaufnahme: 2004, Timpani 1C1077 (Alain Jacquon, Quatuor Louvigny). Noten: Paris: Mutuelle 1911 (via IMSLP). Situation: Satz I: Lent - Modérément animé (T. 19-24), ca. 1.30-1.51 min.

DupOnT, Gabriel (1878-1914): Poème (1911).

Referenzaufnahme: 2003, Timpani 1C1072 (François Kerdoncuff, Quatuor Louvigny). Noten: Paris: Heugel 1911 (via IMSLP). SitUATION: Satz II Claire et calme (Ziffer 5, T. 1-Ziffer 6, T. 1), ca. 3.49-4.21 min.

SCHARWENKA, Philipp (1847-1917): Klavierquintett h-Moll op. 118 (1911). Referenzaufnahme: 1999, MDG 336 0889-2 (Thomas Duis, Mannheimer Streichquartett). NOTEN: Leipzig: Breitkopf \& Härtel 1911 (via IMSLP). SITUATION: Satz II: Adagio con intimo sentimento (T. 1-10), ca. 0.00-0.42 min.

TANEJEW, Sergej (1856-1915): Klavierquintett g-Moll op. 30 (1911).

Referenzaufnahme: 2007, Brilliant Classics 93081/2 (Staffan Scheja, Christiaan Bor, Paul Rosenthal, Rainer Moog, Nathaniel Rosen - Reissue, Aufnahme von 1995). Noten: Berlin: Russischer Musikverlag 1912 (via IMSLP). SituATION: Satz IV: Finale: Allegro vivace (Ziffer 226, T. 1-8), ca. 5.39-5.55 $\mathrm{min}$.

HuRÉ, Jean (1877-1930): Klavierquintett D-Dur (1912).

Referenzaufnahme: 2009, TimPAni 1C1166 (Marie-Joséphe Jude, Quator Louvigny). Noten: Paris: Mathot 1913 (via IMSLP). SituAtion: Satz I (Ziffer 47 bis Takt 9 nach Ziffer 49), ca. 26.42-28.30 min.

RÓŻYCKI, Ludomir (1883-1953): Klavierquintett c-Moll op. 35 (1913).

Referenzaufnahme: 2012, Acte Préable APO253 (Jerzy Godziszewski, Wilanów String Quartet). Noten: Krakau: PWM Edition o.J. ${ }^{301}$ SitUATION: II. Satzes: Adagio, ca. 5.34-8.14 min. 
CATOIRE, Georgy (1861-1926): Klavierquintett g-Moll op. 28 (1914).

Referenzaufnahme: 2009, Aliud ACD HN 033-2 (Rian de Waal, Christian Bor, Boris Tsoukkerman, Michèle Sidener, Godfried Hoogeveen). NOTEN: Moskau: Muzgiz 1921 (via IMSLP). SitUATION: Satz III: Allegro con spirito (Ziffer 60 bis zum Ende), ca. 6.21-7.11 min.

ElgAR, Edward (1847-1934): Klavierquintett a-Moll op. 84 (1918).

Referenzaufnahme: 1997, Naxos 8.553737 (Peter Donohoe, Maggini String Quartet). Noten: London: Novello 1919 (via IMSLP). SitUATION: Satz II: Adagio (Ziffer 35), ca. 5.35-6.32 min.

FRIEDMAN, Ignacy (1882-1948): Klavierquintett c-Moll (1918).

Referenzaufnahme: 2016, Hyperion CDA68124 (Jonathan Plowright, Szymanowski Quartet). Noten: Kopenhagen: Wilhelm Hansen 1918 (via IMSLP). SitUATION: Satz II: Larghetto, con somma espressivo (T. 399-441), ca. 12.00-14.58 $\mathrm{min}$.

MARTIN, Frank (1890-1973): Klavierquintett (1919).

Referenzaufnahme: 1997, ASV CD DCA 1010 (The Britten-Pears Ensemble). Noten: Genf: Henn 1922 (via IMSLP). SitUAtion: Satz III: Adagio ma non troppo (T. 7 vor Ziffer 50 bis Ziffer 60), ca. 3.17-4.19 min.

ZILCHER, Hermann (1881-1948): Klavierquintett cis-Moll op. 42 (1918).

ReferenZaufnahme: 1999, Largo 5144 (Carl-Heinz März, Nicolay Quartett). Noten: Leipzig: Breitkopf \& Härtel 1919 (via IMSLP). SituAtion: Satz I: Leidenschaftlich bewegt (Ziffer 7), ca. 2.56-3.35 min.

PIERNÉ, Gabriel (1863-1937): Klavierquintett e-Moll op. 41 (1919).

Referenzaufnahme: 2000, Musidisc 365 807-2 (Akiko Ebi, Quatuor Parisii). Noten: Paris: Hamelle 1919 (via IMSLP). SituAtion: Satz II: Sur un rythme de Zortzico (Ziffer 31, T. 1-8), ca. 1.22-1.32 min.

BAX Arnold (1883-1953): Klavierquintett g-Moll GP 167 (1920).

Referenzaufnahme: 1990, Chandos Chan 8795 (David Owen Norris, The Mistry Quartet). Noten: London: Murdoch 1922 (via IMSLP). SituAtion: Satz III: Moderate tempo (T. 59-75), ca. 2.25-3.05 min.

MADDISON, Adela (1862-1929): Klavierquintett (1920).

Referenzaufnahme: 2009, Dutton Epoch CDLX 7220 (The Fibonacci Sequence - Ersteinspielung). NOTEN: London: Curwen 1925. SituATION: Satz IV: Allegro vivo, ca. 2.14-3.09 $\mathrm{min}$. 
FAURÉ, Gabriel (1845-1924): Klavierquintett Nr. 2 c-Moll op. 115 (1921). Referenzaufnahme: 1986, Claves CD 50-8603 (Quintetto Fauré di Roma). Noten: Paris: Durant 1921 (via IMSLP). Situation: Satz III: Andante moderato (T. 4 nach Ziffer 4), ca. 3.20-3.22 min.

KoEChlin, Charles (1867-1950): Klavierquintett op. 80 (1921 - UA erst 1934; Veröffentlichung 1985).

Referenzaufnahme: 2013, AR Ré-Sé AR20091 (Sarah Lavaud, Antigone Quartet). Noten: Paris: Eschig 1985 (via IMSLP). SituATION: Satz III: La Nature consolatrice... Andante, très calme, lent, trés doux (T. 4-8 [bis Ziffer 1]), 0.39-1.12 $\mathrm{min}$.

CRAS, Jean (1879-1932): Klavierquintett C-Dur (1923).

REFERENZAUfNAHME: 2001, Timpani 1C1066 (Alain Jacquon, Quatuor Louvigny). Noten: Paris: Senart 1923 (via IMSLP). SitUATION: Satz II: Calme et paisible (Ziffer 20 bis T. 8 nach Ziffer 21), ca. 4.55-6.16 min.

HAHN, Reynaldo (1874-1947): Klavierquintett fis-Moll (1923).

Referenzaufnahme: 2001, Hyperion CDA67258 (Stephen Coombs, Chilingirian Quartet). NOTEN: Paris: Heugel 1923 (via IMSLP). SituATION: Satz II: Andante (non troppo lento) (Ziffer 4, T. 1-20), ca. 3.29-4.17 min.

KoRNGOLD, Erich Wolfgang (1897-1957): Klavierquintett E-Dur op. 15 (1923).

Referenzaufnahme: 1992, Marco Polo 8.223385. Noten: Mainz: Schott 1924 (via IMSLP). SitUATION: Satz I: Mässiges Zeitmass, mit schwungvoll blühendem Ausdruck (T. 1-11), ca. 0.00-0.26 min.

D’INDY, Vincent (1851-1931): Klavierquintett g-Moll op. 81 (1924).

Referenzaufnahme: 1995, Marco Polo 8.223691 (Ilona Prunyi, New Budapest Quartet). Noten: Paris: Senart 1925 (via IMSLP). Situation: Satz IV: Modérément animé (Ziffer 40, T. 1-4), ca. 3.24-3.32 min.

SCHNABEL, Alexander Maria (1889-1969): Klavierquintett C-Dur op. 17 (1925).

Referenzaufnahme: 2015, Cornetto COR10041 (Malinconia-Ensemble Stuttgart). Noten: o.A. SitUATION: Satz II: Breit und gesangvoll, mit tiefer Empfindung, ca. 4.14-4.31 min. 
KORNAUTH, Egon (1891-1959): Klavierquintett op. 35a (1933 - Eigenbearbeitung der Orchestersuite op. 35).

ReferenZaufnahme: 2009, Cavalli CCD 276 (Andreas Kirpal, Diogenes Quartett). Noten: Wien: Doblinger 1951. SituAtion: II. Satz: Notturno: Moderato, ca. 0.00-0.45 min.

ENESCU, George (1890-1959): Klavierquintett a-Moll op. 29 (1940 - Umarbeitung bis mindestens 1949, UA erst posthum 1964).

Referenzaufnahme: 2003, Naxos 8.557159 (The Solomon Ensemble). Noten: Bucharest: Editura Muzicală a Uniunii Compozitorilor din R.P.R. 1968 (via IMSLP). SituAtion: Satz II: Andante sostenuto e cantabile (Ziffer 16, T. 16-20), ca. 0.00-0.20 min.

Schostakowitsch, Dimitri (1906-1975): Klavierquintett g-Moll op. 57 (1940).

ReFerenZaufnahme: 2019, Erato 0190295540760. Noten: Moskau: DSH 2014. SituAtion: Satz III: Scherzo: Allegretto, ca. 1.10-1.21 min.

MARTINU゚, Bohuslav (1890-1959): Klavierquintett Nr. 2 H. 298 (1944).

ReferenZaufnahme: 2007, Naxos 8.557861 (Karel Košárek, Martinů Quartet). Noten: New York: Schirmer o.J. Situation: Satz II: Adagio, ca. 3.31$4.20 \mathrm{~min}$.

MedTNER, Nikolai (1880-1951): Klavierquintett C-Dur op. posth. (1950 Arbeiten seit 1904).

ReferenZaufnAHME: 1997, Naxos 8.553390 (Konstantin Scherbakov, Ewald Danel, Milan Tedla, Zuzana Bouřova, Jozef Podhoranský). Noten: Moskau: Muzgiz 1963 (via IMSLP - Erstausgabe Frankfurt am Main: Zimmermann 1955, jetzt über Schott zu beziehen). Situation: Satz I: Molto placido (Buchstabe G, T. 11-19), ca. 5.39-6.60 min.

BACEWICZ, Grażyna (1909-1969): Klavierquintett Nr. 1 (1952).

Referenzaufnahme: 2011, Deutsche Grammophon CD DDD 0289477 83329 GH (Krystian Zimerman, Kaja Danczowska, Agata Szymczewska. Ryszard Groblewski, Rafal Kwiatkowski). NOTEN: Krakau: Polskie Wydawnictwo Muzyczne 1953. SituAtion: Satz III: Grave, ca. 2.26-2.36 min.

GuBAIDULINA, Sofia (*1931): Klavierquintett (1958).

Referenzaufnahme: 2002, BIS BIS-CD-898 (Rieko Aizawa, Kai Vogler, Mira Wang, Ulrich Eichenauer, Peter Burns). Noten: Hamburg: Musikverlag Hans Sikorski 1990. SitUATION: Satz I: Allegro, ca. 5.04-5.35 min. 
SCHNITTKE, Alfred (1934-1998): Klavierquintett op. 108 (1976).

REFERENZAUfNAHME: 2003, ECM 4618152 (Alexei Lubimov, Keller Quartett). Noten: Hamburg: Musikverlag Hans Sikorski 1976. SituAtion: Anschluss des Endes des Werks an seinen Beginn und damit die zirkuläre Konzeption des Stücks.

FeldMan, Morton (1926-1987): Piano and String Quartet (1985).

Referenzaufnahme: 1993, Elektra Nonesuch 7559-79 320-2 (Aki Takahashi, Kronos String Quartet). NOTEN: Wien: Universal Edition 1995. SITUATION: Satz I, ca. 42.48-1.19.38 min.

LiEBERMANN, Lowell (*1961): Klavierquintett op. 34 (1991).

ReferenZaufnahme: 2008, Koch International Classics 7743 (David Korevaar, Elizabeth Kipper, Margaret Soper Gutierrez, Matthew Dane, Thomas Heinrich. Noten: King of Prussia/PA: Theodore Presser 1992. Situation: Satz III: Molto adagio, ca. 1.51-2.49 min.

EBEN, Petr (1929-2007): Klavierquintett (1992).

ReferenZaufnahme: 2017, Supraphon SU 4232-2 (Karel Košárek, Martinů Quartet). Noten: Kassel: Bärenreiter o.J. SituAtion: Satz II: Allegretto, ca. 0.00-0.30 min.

VENABLES, Ian (*1955): Klavierquintett op. 27 (1996).

ReFerenZAufNAHME: 2010, SOMM SOMMCD 0101 (Mark Bebbington, Coull Quartet). Noten: Eigenverlag. ${ }^{302}$ SituAtion: Satz I: Molto adagio e espressivo - Allegro ma non troppo, ca. 3.32-4.13 min.

GATES, Philip (*1963): Klavierquintett (1997).

Referenzaufnahme: 2008, Melodist 3130CD (Philip Gates, Carducci Quartet). NOTEn: Eigenverlag (via http://www.philipgates.com/). SituATION: Satz II: Grazioso, ca. 0.39-0.55 min.

KAPUSTIN, NiKOLAI (*1937): Klavierquintett op. 89 (1998).

ReferenZaufnahme: 2004, Triton OVCT-00011 (Nikolai Kapustin, Vladimir Spektor, Alexander Chernov, Svetlana Stepchenko, Alexander Zagorinsky). Noten: Berlin: Boosey \& Hawkes o.J. Situation: Satz I: Allegro, ca. 4.27$4.40 \mathrm{~min}$.

ADÈS, Thomas (*1971): Klavierquintett op. 20 (2001).

Referenzaufnahme: 2005, EMI Classics 724355766427 (Thomas Adès, Arditti Quartet - Uraufführungsbesetzung). ${ }^{303}$ NOTEN: London: Faber Music 2007. SituATION: Satz II (Ziffer 18), ca. 3.58-5.05 min. 
GaAthaug, Morten (*1955): Klavierquintett fis-Moll op. 59 (2005).

RefEREnZAufnAhME: 2007, 2L 2L44SACD (Tore Dingstad, Ensemble Bjørvika). Noten: NB Noter: Oslo o.J. SituAtion: Satz III: Adagio, ca. 0.48-1.09 $\min$.

ABril, Antón García (*1933): Alba de Los Caminos (2007).

Referenzaufnahme: 2009, Verso VRS 2075 (Daniel del Pino, Cuarteto Leonor). ${ }^{304}$ Noten: Madrid: Bolamar Ediciones Musicales 2007. SitUATION: Satz II: Allegro brioso (T. 1-15), ca. 0.00-0.20 min.

FrIES, Albin (*1955): Klavierquintett Nr. 1 h-Moll op. 10 (2007).

ReFerenZaufnahme: 2011, Eigenverlag (Gerhard Schlüsslmayr, Hibiki Quartet). ${ }^{305}$ NOTEN: Eigenverlag (via IMSLP). SitUATION: Satz II: Andante espressivo (T. 75-89), ca. 5.05-6.08 min.

Gourzi, Konstantia (*1962): Vibrato 1 und Vibrato 2 (2010).

ReferenZAufnAhME: 2014, ECM 2309 (Lorenda Ramou, Ensemble Coriolis). Noten: München: Musikproduktion Höflich 2010. SitUAtion: Vergleich der Differenzen in den Klavierpartien, deren Verteilung bei gleichbleibendem Streichersatz unterschiedlich ist, in I in traditionellem Sinne festgelegt, in II innerhalb eines vorgegebenen Rahmens frei.

PiCKER, Tobias (*1954): Live Oaks (2011).

ReferenZaufnahme: 2014, Livemitschnitt via YouTube (Aaron Likness, Fidelio String Quartet). ${ }^{306}$ Noten: Mainz: Schott 2011. Situation: Satz II (T. 24-28), ca. 27.45-28.04 $\mathrm{min}$.

FriedMAN, Jefferson (*1974): The Heart Wakes (2014).

REFERENZAUfNAHME: 2014, Livemitschnitt der Uraufführung via YouTube (Simone Dinnerstein, Chiara String Quartet). ${ }^{307}$ NotEN: Eigenverlag. SitUATION: Satz VI: Is having, had, departed, ${ }^{308}$ ca. $23.11-23.33 \mathrm{~min}$.

IYER, Vijay (*1971): Time, Place, Action (2014).

Referenzaufnahme: 2014, Livemitschnitt via YouTube (Vijay Iyer, Brentano Quartet). ${ }^{309}$ Noten: Mainz: Schott 2014. SituAtion: Satz III, ca. 7.11-7.32 $\mathrm{min}$.

SøREnSEn, Bent (*1958): Rosenbad-Papillons (2014).

REFEREnZAufnAhme: 2018, DACAPO RECORDS 8.226135 (Katrine Gislinge, Stenhammar Quartett - Uraufführungsensemble). NOTEN: Kopenhagen: Wilhelm Hansen 2013. SituAtion: Satz II: Calmo con delicatezza, ca. 0.59-1.06, 1.54-2.08, 2.54-3.08, 3.54-4.50 $\mathrm{min}$. 
SAY, Fazil (*1970): Yürüyen Köşk. Hommage à Atatürk (2017).

Referenzaufnahme: Es liegt noch keine Aufnahme vor. Noten: Mainz:

Schott 2017. SituATiON: Satz I: Enlightenment (T. 1-9).

\section{VERORTUNGEN: ANNÄHERUNGEN AN AUSGEWÄHLTE SitUATIONEN}

Im Anschluss an den Exkurs des vorangegangenen Kapitels wird im Folgenden stellvertretend für gut zwei Dutzend Ausstellungsgegenstände die Verortung des »emotional impact» in der Musik, sein konkreter Bezugspunkt im Gehörten nicht nur über Angaben zu Tonaufnahmen und Noten offengelegt und darin auffindbar gemacht, sondern nun auch näher beschrieben. Eine Auswahl genügt für hiesigen Zweck. Weswegen ich es dabei belasse. Der Punkt, um den es mir hier geht, ist damit gemacht: Digital-Humanities-Forschung im Bereich der Musik wie das erwähnte Projekt Dig That Lick erlaubt es einem nämlich insbesondere, davon ausgehend sehr präzise weiter zu fragen. Dort wird aus einer enormen, Jahrzehnte überspannenden Jazzproduktion extrahiert, wo sich jeweils dieselben Licks in Soli wiederfinden. ${ }^{310}$ Man kann plötzlich, mit Jannidis gesprochen, auch weit jenseits des allgemein bekannten Kernrepertoires an Jazzmusikern und Jazzalben nach Gemeinsamkeiten und Unterschieden, aber vor allem nach den musikalischen Kontexten fragen, in denen die jeweiligen Licks auftauchen. Das Spannendste für mich an solchen digitalen Projekten ist aber eben dies, dass sie einem nämlich Türen öffnen. Sprungbretter sind. Das freilich ist genau das, was das Bemühen, den besagten »emotional impact« in den Klavierquintetten zu verorten, aus ganz anderer Richtung kommend für mich in gleicher Weise leistet: Ungleich genauer von hier aus Kontexte erkunden und in ihrer Rolle für den jeweiligen Anlass - Lick hier, »emotional impact« dort - befragen zu können. Das Folgende ist also kein Ergebnis. Es ist ein Ausgangspunkt. To be continued...

\section{Johannes Brahms (1833-1897): \\ Klavierquintett f-Moll op. 34 (1864)}

Der II. Satze Andante, un poco adagio verharrt in Brahms' Klavierquintett fast schüchtern zwischen einem mächtig dramatischen Allegro non troppo 
und einem rasanten Scherzo: Allegro. ${ }^{311}$ Es ist der Teil, der für mich am meisten nach Schubert klingt. Jenem der schönen Müllerin. Dessen 1853 posthum veröffentlichtes Streichquintett in C-Dur D 956 war in seiner Besetzung mit zwei Celli Ausgangspunkt für jenes verschollene Streichquintett gewesen, mit dem Brahms 1862 das Projekt begann, dessen komplizierter Weg über die Fassung für zwei Klaviere op. 34b Ende 1864 schließlich zum Klavierquintett op. 34 führte. Eher einfache Musik ist dieser II. Satz. Eher Volkston, wie man früher sagte. Hier und da eine schärfere Eintrübung in Moll. Hier und da etwas slawisches Kolorit. Hier und da ein kurzer dramatischer Impuls. Aber doch vor allem schlicht. $\mathrm{Zu}$ rückgenommen. Zart. Viele Dreiklänge. Viel Stufenharmonik. Viele Terzund Sextharmonisierungen der Melodie. Der Kontrast zu den beiden das Andante umrahmenden Sätzen könnte kaum größer sein.

Ganz am Schluss (T. 118 mit Auftakt bis T. 126, ca. 24.02-24.45 min) erscheint gleichfalls in einfachster, innerhalb der Schlüsseltakte 120/121 changierender Harmonisierung (Tonika/I./As-Dur - Subdominantparallele/II./b-Moll | Subdominantparallele/II./b-Moll - Tonika/I.) über dem hier als Orgelpunkt durchgehend wiederholten Tonikagrundton as eine viertönige motivische Figur, die bis dahin bereits mehrfach zu hören war. Immer wieder ansetzte. Aber hier nun über 4 Takte zum Strahlen gebracht wird. Durchbricht. Aufblüht. Das erste Mal im Satz frei atmen darf. Um doch genauso rasch wieder zu verklingen. Zurückgenommen zu werden, um den Satz eine Handvoll Takte später schon zu beschließen. Wie ein Aufleuchten von dem, was möglich gewesen wäre. Eine Geste. Ein Blick. Ein Sehnen. Und vorbei. Chance vertan.

Es ist die Schärfe der Intonation in der Referenzaufnahme in den Sexten der Violinen und dem Nachhallen der Bratsche, wenn die Passage für 2 Takte mit ihren Vorhalten auf schwerer Schlagzeit ihren Höhepunkt erreicht, die diese Interpretation für mich aus den Legionen von Aufführungen und Aufnahmen herausstechen lässt, die ich von diesem Werk kenne. Die mir diese Passage überhaupt erst als »emotional impact« erschlossen hat. ${ }^{312}$ Es klingt für mich, als hörte man für einen Moment nicht fünf Musiker. Sondern einen. Der im Hier und Jetzt empfindet. Erlebt. Artikuliert. Keine Darbietung von etwas, das jemand anderes sich ausgedacht hat. Und dies vor langer Zeit. Sondern etwas, das einem selbst passiert. Gerade. In diesem einen Augenblick. Intonation und Binnendynamik der Interpretation der einzelnen Stimmen: Das macht es hier. 


\section{Marie-Alexis de Castillon de Saint-Victor (1838-1873): Klavierquintett Es-Dur op. 1 (1864)}

Castillon ist ein wenig der >Frédéric Bazille der französischen Klassischen Musik um 1870. ${ }^{313}$ Seine wenigen Werke sind dabei Robert Schumanns Romantik ungleich näher als z.B. Camille Saint-Saëns' Klassizismus in dessen für das französische Klavierquintett $\mathrm{zu}$ dieser Zeit so zentralen Komposition in a-Moll op. 14, ein Jahrzehnt vor Castillons ca. 1863-64 komponiertem op. 1 entstanden. Aber es ist auch weit weg von Brahms' zeitgleich verfasstem op. 34. Die Schlüsselstelle seines Werks steht stellvertretend hierfür: Wenn bei Castillon die Passage etwa zur Mitte des als zweites folgenden Scherzos erscheint, um die es mir geht (Satz II, 5 Takte nach Buchstabe D, T. 278-300, ca. 3.48-4.25 min), hat man bereits ein leidenschaftliches, kontrastreiches Allegro gehört. Dessen ausnehmend sangliches, sehnsuchtsvolles Hauptthema von großem Nachhall prägt einen Satz, der durch unterschiedlichste Stimmungen und Register getrieben wird. An den konstant präsenten motorischen Impuls des Allegros schließt das Scherzo unmittelbar an, aber nicht an die lyrische Kraft des Hauptthemas, sondern an das Majestätische des dortigen Nebenthemas.

Nichts an der zunächst mehrfach aufgerufenen, geradezu herausfahrenden, fast schroffen Gestik des das Scherzo eröffnenden Themas, noch an der den Kontrast dazu bildenden kleinteiligen, pointiert betonten raschen Motorik bereitet einen freilich auf die Romantik des Moments vor, der ab Buchstabe D folgt. Romantik im Sinne von Eichendorffscher Mondnacht, nicht von Hoffmannscher Groteske oder Heinescher Ironie. Die Musik verdunkelt sich schlagartig von C-Dur nach c-Moll. Doch das Satzbild lichtet sich zugleich über diesen Wechsel hinweg. Der so mehr wie eine sachte Eintrübung wirkt. Parallel wird eine Pendelbewegung vorbereitet, welche schließlich in der linken Hand des Klaviers die ganze, ab Tonartwechsel 28-taktige Passage grundiert, ab dem Moment, ab dem die Melodie einsetzt. Cello und zweite Geige ziehen sich für den Augenblick auf sparsame gemeinsame, nur flüchtig hingetupfte Pizzicato-Akkordbrechungen zurück. Die Bratsche hingegen beginnt, parallel die vom Klavier in mittlerer Lage geführte Basslinie der nun von c-Moll aus einsetzenden, recht weichen, starke Affinität zur Dur-Paralleltonart Es-Dur zeigenden harmonischen Progression dieses Abschnitts (c7/b - As6 - B7/as - c7/g - f7 - B7/fes-f-es) zu betonen und zugleich den für die Wirkung der Melodie in dieser 
Passage wesentlichen, genau hier einsetzenden Wechsel zwischen 3/4-Taktund 4/4-Takt-Phrasen hervorzuheben. Bei diesem Spiel mit metrischen Kontrasten überlagert sich die Harmoniefortschreitung (im steten Wechsel 3/4-4/4-Takt) mit der stets auf Schlag einsetzenden, viertaktigen Melodiephrase, die insgesamt zweimal in der ersten Violine und einmal in der rechten Hand des Klaviers in unterschiedlicher Lage erscheint. Und so metrisch etwas überlappt. Leise Instabilität macht sich auf diese Weise breit. Etwas Suchendes. Ein scharfer Gegensatz zur groben Bestimmtheit des Hauptthemas dieses Satzes. Doch die Melodie ist keineswegs neu: Es ist genau dieses grobe, großspurige Hauptthema. Doch nun zart, vorbeihuschend, melancholisch getönt, nicht recht wissend, wo es hin will. Hin gehört. Welch ein Kontrast! Welch ein Effekt! Hat man dies erst einmal erkannt, kann man das Hauptthema des Satzes mit seinem fast schon herrischen Habitus nie mehr so eindimensional hören wie es sich doch eigentlich mit aller Kraft zu geben versucht. So erkundet dieses Scherzo letztlich, welch maximale Gegensätze im Ausdruck in derselben melodischen Phrase liegen.

\section{Giovanni Sgambati (1841-1914): Klavierquintett Nr. 1 f-Moll op. 4 (1866)}

Fünf Akkorde beschließen in Des-Dur den III. Satz von Giovanni Sgambatis erstem Klavierquintett in f-Moll op. 4: Des-Dur - b-Moll - Ges-Dur maj7 - As-Dur 6/7 - Des-Dur. ${ }^{314}$ Die Akkorde schreiten ab T. 20 nach Buchstabe $\mathrm{E}$ in Halben gemächlich voran, der erste Akkord Des schon aus T. 19 kommend. Feierlich. Der Ton f liegt durch, in der 1. Violine und in den Akkordlagen im Klavier. Er bestimmt das Flair der Progression. Denn mit jedem Akkordwechsel erscheint er in neuer Farbe, in anderer Funktion im Akkord: Terz - Quinte - große Septime - große Sexte - Terz.

In diese Gleichmäßigkeit prescht die Bratsche mit ihrem vorletzten Ton. Ein Quartsprung rauf in die Septime der Dominante. Von des nach ges. Nur ein Quartsprung rauf. In einen erwartbaren Akkordton. Des vorhersehbarsten Akkords überhaupt an dieser Stelle. Vom Quintton der Subdominante in den Septimton der Dominante. Aber Musik ist Kontext, Kontext, Kontext. Und dieser verleiht dem ges der Bratsche an dieser Stelle nicht nur maximale Aufmerksamkeit. Der Zusammenhang zwingt diesen Ton zudem in eine Reibung mit dem durchliegenden Ton $\mathrm{f}$. Das zieht das ges schon in 
die Richtung von jenem $\mathrm{f}$, in das es sich auch sogleich auflösen wird. Eine Septime eines Dominantseptakkords mit der Schärfe und Energie eines Quartvorhalts zur Tonika.

\section{Giuseppe Martucci (1856-1909): Klavierquintett C-Dur op. 45 (1878)}

Giuseppe Martuccis einziges Klavierquintett wurde 1877 in einer beruflich wichtigen Phase komponiert und gilt in Italien gemeinhin als erstes reifes Meisterwerk des damals ja erst in seinen frühen 20er Jahren stehenden Komponisten. ${ }^{315}$ Uraufgeführt am 17. März 1878, gewann er damit den prestigeträchtigen Mailänder Kammermusikwettbewerb der Società del Quartetto. Dem ungeachtet wurde das Werk erst anderthalb Jahrzehnte später, dann aber im für Kammermusik zentralen deutschsprachigen Markt bei Kistner in Leipzig verlegt. Vorausgegangen war eine einschneidende Revision durch den Komponisten zu Beginn der 1890er Jahre. Kaum ein Werk eignet sich besser, die spezifischen Qualitäten von Martuccis Musik vorzustellen, als sein op. 45. Elegante Formbehandlung. Ausgesprochen sanglich gehaltene Melodik einschließlich prägnant-akzentuierter Themen. Vielfarbige, spätromantische, den tonalen Rahmen nie herausfordernde Harmonik reich an Sext-, Sept- und Nonakkorden und effektvollen Vorhaltbildungen, jedoch unter Verzicht auf exponierte chromatische Stimmführung, scharfe Brüche oder grobe Kontraste. Kunstvolle, dabei nie aufdringlich-plakative Instrumentierung. Breites Charakterspektrum von sonnigem Überschwang über leichtfüßig-tänzerischen Gestus bis hin zu sanfter, nie depressiver Melancholie. Ausgewogene, in ihren Proportionen konzentrierte, in sich ruhende Musik.

Immer wieder bricht Martucci den vollen Satz aller fünf Instrumente auf. So auch an der Stelle, die mich hier interessiert. Sie erscheint im zweiten Satz Andante con moto bei Buchstabe B nach einem guten Drittel der Aufführungsdauer dieses Satzes (T. 80-91, ca. 3.41-4.09 min). Vorausgegangen ist bis dahin in diesem Satz ein langer Abschnitt (T. 1-49), der bei aller melodischer Prägnanz eher suchend, vorbereitend wirkt. Wie eine Einleitung. In T. 50 hebt das Hauptthema des Satzes an. Majestätisch. Getragen. Zunächst nur durch das vom Klavier begleitete Cello vorgetragen. Ein zweiter Durchlauf durch das Thema folgt im vollen Ensemble. Ein zweitaktige Triolenfigur (T. 78f.) kehrt wieder, die bereits als Überlei- 
tungselement zu hören war, korrespondierend vor Beginn von Buchstabe A (T. 29f.). Sie markiert den Anfang besagter Passage. Das neue Thema, das nun mit Buchstabe B (T. 80) anhebt, wird gleichfalls hiernach im ganzen Ensemble behandelt werden. Seine Vorstellung erfolgt jedoch zunächst in der Bratsche, begleitet nur von den beiden Violinen, die drei- bis vierstimmige Akkorde beisteuern. Im Piano, leiser gehalten als das Mezzoforte der Bratsche. Der 3/4-Takt wird zum 9/8-Takt verdichtet.

Das ist die für mich anmutigste Passage, die im ganzen Buch besprochen wird. Der Charakter des Themas verändert sich zu erhabener Größe, wenn es wenig später (ab T. 97) von der ersten Violine bei voller Streicherbegleitung angestimmt wird, dann eine Dezime höher. Bei seiner Einführung wenige Takte zuvor stellt es sich jedoch geradezu schüchtern vor. Wie fragil diese Anmut bei der ersten Vorstellung des Themas ist, wie schwer in der Interpretation zu erzeugen, hört man freilich deutlich in der Gegenüberstellung der weiteren Aufnahmen des Werks mit der angegebenen Referenzaufnahme. ${ }^{316}$ Die Begleitung der Violinen muss trotz der Mehrstimmigkeit federleicht klingen. Kaum mehr als ein Schatten sein, damit ich diese Passage als anmutig erfahre. Die erhebliche technische Herausforderung dieser beiden Partien hat völlig zurückzutreten. Die vermeintliche Hauptsache, die Melodie, steht also im Vordergrund. Ist aber gar nicht entscheidend für mein ästhetisches Erleben dieser musikalischen Situation.

\section{Christian Sinding (1856-1941): Klavierquintett e-Moll op. 5 (1885)}

Andere schnelle Sätze, die in diesem Buch aufgegriffenen werden, so wie jene in Castillons op. 1 oder Dvořáks op. 81, Nováks op. 12 oder Schostakowitschs op. 57 ziehen sehr viel ihrer Ausdruckskraft aus dem Kontrast der konträren Ausdruckswelten tänzerischer und lyrischer Passagen. Nicht so Christian Sindings einziges Klavierquintett aus den 1880er Jahren, Ende 1885 in Kristiania (heute Oslo) uraufgeführt. ${ }^{317}$ Einem der selten Fälle, in denen es ein Klavierquintett war, das den Komponisten bekannt machte. Das hier an dritter Stelle stehende Vivace ist ein Reigen ohne Pause. Variation wird über den Wechsel von vollstimmigem und lichtem Satzbild gewonnen sowie über den Kontrast von laut und leise. Rhythmisch akzentuiert bleibt die Musik dieses an Wiederholungen reichen, Intermezzo überschriebenen Satzes jedoch die ganze Zeit. Selbst in den kurzen Momenten, 
wenn die Musik wie nach den ersten beiden Durchläufen durch das Hauptthema kurz neu ansetzt, um neuen Schwung aufzunehmen. Der Gegensatz könnte nicht größer sein zu dem elegischen, kaum extrovertierte Emotion zeigenden Andante, welches für gut 10 Minuten Aufführungsdauer unmittelbar zuvor erklingt. Schöne Musik. Aber über weite Strecken eben eher zurückhaltend. Mit einer Ausnahme, einer sich ab T. 9 (mit Auftakt) nach Buchstabe C nach immerhin schon gut $60 \%$ Spielzeit (ca. 6.11-8.07 min) jenes Satzes sehr langsam aufbauenden, konzertanten, aber doch auch hier im Gestus majestätisch-getragenen Steigerung. Diese löst sich zudem unversehens in den verbleibenden gut 2 Minuten Aufführungsdauer wieder auf. Es bleibt nur Nachhall. In Zeitlupe. An zuvor bereits gehörtes thematisches Material. Und wenn man sieht, dass für diese verbleibenden gut 2 Minuten in der Partitur nicht einmal eine Seite vorgehalten wird, ist sofort klar, wie ernst, d.h. langsam das Più Lento hier gemeint ist, das Sinding vorschreibt.

Das hat für sich stehend schon eine starke Ausdruckskraft. Aber vor allem einen satzübergreifenden Wert. Im Kontrast zum Vorangegangenen wirkt der Beginn des Vivace fast wie eine Erlösung. Wie nach einem langen Vortrag. Wenn einen der Applaus urplötzlich aus dem Versunkensein und dem Wandern der Gedanken reißt, man sich unversehens strafft, durchamtet - und los geht es. Das achttaktige Hauptthema setzt dabei im Streichquartett unmittelbar ein und wird zwei Mal wiederholt. Leggiero ist das Motto: Leicht, spielerisch. Die ersten 16 Takte bleiben durchgehend auf dem Tonikagrundton g. Die harmonische Progression ist dabei langsam. Alles licht gehaltenes G-Dur. Für 12 Takte. Die feine, zirkuläre Chromatik über die übermäßige Quinte d - dis - e - dis in den Takten 5-8 bleibt ganz Nuance. Dann puscht die Subdominanteparallele a-Moll (über g) noch etwas die Energie. Diese Energie wird weitergeführt. Das Sprungmotiv, in welches das Hauptthema mündet (T. 5 bzw. 12), wird zum Ausgangspunkt der weiteren melodischen Gestaltung. Die Bewegung der raschen, großräumigen Achtelakkordbrechungen (T. 5-8, 12-15), die dem Sprungmotiv zuvor unterlagen, werden gleichfalls weiterverwendet. An die Stelle des Orgelpunkts $\mathrm{g}$ tritt jedoch eine absteigende, taktweise fortschreitende, ziemlich markante Basslinie in der linken Hand des Klaviers (fis $-\mathrm{e}-\mathrm{d}-\mathrm{c}$ $-\mathrm{h}-\mathrm{b}-\mathrm{a}-\mathrm{as}-\mathrm{g}$ ) über die Akkordfolge D6/fis - C/e $-\mathrm{D}-\mathrm{C}-\mathrm{G} / \mathrm{h}-\mathrm{B}^{\mathrm{o}} 7$ - D7/9/a - D7/55/as. Die chromatische Reibung, welche die Akkordsymbole andeuten, bleibt jedoch auch hier dezent. Mit dem Zielbasston g wird 
eine kleine, zweigeteilte, achtteilige Sequenz erreicht (G - G6 - A9/g D/fis | A/fis - fis6 - D6/fis - A7/e), die wiederum in das besagte Sprungmotiv mündet. Eine achttaktige Steigerung mit diesem führt zurück zum Hauptthema, nun von Klavier und Streichquartett mit vertauschten Rollen und auf der Stufe der Subdominantparallele ausgeführt. Ein kleines Ausmäandern über das Kopfmotiv des Hauptthemas. Und schon setzt der ganze, insgesamt 64-taktige, ganz gleichmäßig gegliederte Eröffnungsabschnitt zur Wiederholung an.

Den Höhepunkt erreicht die hier unmittelbar, ohne jeden Vorlauf ansetzende, stets spielfreudige und obendrein ausgesprochen eingängige Musik mit Griegschem Flair nach fast 6 Minuten Aufführungsdauer, kurz vor Schluss. 540 Takte (einschließlich Wiederholungen) wurden bereits durchlaufen, wenn man diesen Höhepunkt schließlich erreicht (ca. 5.46-5.59 min). Der letztlich nicht mehr ist als ein über mehrere Takte gestreckter Dominant-Sept-Non-Akkord in G-Dur mit mehrfach angespieltem und aufgelöstem Quart-Sext-Vorhalt, der in einem Solotriller des Klaviers auf d endet. Eine kleine Spannung kommt noch dadurch hinzu, dass die Akkordbrechungen der Streicher im 6/8 gegen die Betonungen des Klaviers im 3/4-Grundmetrum des Satzes stehen.

Aber wie so oft bei den Stellen, die in diesem Buch herausgegriffen werden, ist es eigentlich der Kontext, der die besondere Wirkung bringt. Hiesige Passage ist nämlich eine Verlängerung jenes eben beschriebenen, mitreißenden Hauptthemenabschnitts, mit dem das Intermezzo unmittelbar begonnen und den man bis zu diesem Moment bereits vierfach gehört hatte. Der durch diesen neuen Binnenschluss aber nochmal an Emphase gewinnt, indem die Musik nun von subdominantischer auf dominantische Ebene gezogen und um eine verlängerte Schlussformel erweitert wird, die das Ganze nochmals betont. Die Musik jubiliert an dieser Stelle, so laut sie kann. Mit breitem, jugendlichen Lachen.

\section{Antonín Dvořák (1841-1904): Klavierquintett Nr. 2 A-Dur op. 81 B. 155 (1888)}

Dvořáks Klavierquintett Nr. 2 entstand im Spätsommer/Frühherbst 1887.318 Es wurde in Prag schon wenig später am 6. Januar 1888 uraufgeführt und noch im selben Jahr bei Simrock in Berlin als Noten herausgegeben. Es entstand in unmittelbarer Reaktion auf intensive Bemühungen, sein erstes 
Klavierquintett op. 5 umzuarbeiten, das die Tonart A-Dur mit op. 81 teilt. Mit Dumka und Furiant als Bezeichnungen der Mittelsätze ist das Stück zugleich offen ausgewiesen darin, zu jenem Teil des Schaffens von Dvořák zu gehören, in dem er sich mit slawischer Volksmusik als Inspirationsquelle auseinandersetzt. Die Melodien wie die ganze Gestaltung sind ganz seins. Aber bestimmte Elemente aus dieser lokalen Alltagskultur in Form, Rhythmus und Ausdruck dienen als Ausgangspunkt jener Musik. Werden aber unter den Bedingungen intellektueller, höchst origineller und oft virtuoser Klassischer Musik neu gedacht und auf die Reichweite ihres expressiven Potentials hin befragt. Freilich bleibt viel der Unmittelbarkeit dieser musikalischen Alltagspraktiken erhalten. Ein Teil des Charmes dieser Musik für mich. Der III. Satz ist die wahrscheinlich fröhlichste Musik in diesem Buch. Dass die gewählte Form Furiant einen Volkstanz als Bezugspunkt hat, ist die ganze Zeit über präsent. Wenn Furiant tschechisch wörtlich »Der Begeisterte« heißt, ist Dvořáks Satz der Kronzeuge hierfür. Welch gute Laune.

Auf halbem Weg hat Dvořák freilich 28 Takte Poco Tranquillo eingefügt (ca. 1.27-1.50 min). Ein kurzes Luftholen. Ein federleichtes Hin- und Herwiegen. 12 Takte ganztaktig ausgehaltene Klavierakkorde beginnen, pendelnd zwischen Tonika und Subdominante. Die Energie der Musik zuvor wird jedoch gehalten, da der Streichersatz wie Akkordbrechungen konfiguriert ist. Der rhythmische Puls treibt so im Untergrund voran, obwohl das Klavier aus der Sache erkennbar Luft herauszunehmen versucht. Nach 8 Takten erscheint dabei das Hauptthema des Satzes wieder. Als Erinnerung huscht es leise durch die drei oberen Streicher. Diese münden in Akkordflächen des Quartetts. Hier nun kommt der Clou. Die puristische harmonische Progression wird wiederholt. Aber anders inszeniert. Die rechte Hand des Klaviers bricht über den Streicherflächen die Akkorde glockenklar. Die Melodie freilich ist in die linke Hand des Klaviers gewandert. Und wird dort gleichmäßig in Halben inszeniert. Freilich erscheinen diese Halben im 3/4-Metrum immer erst auf der 2. Schlagzeit. Die ganze Passage erhält hierdurch ein Off-Beat-Feel. Was im Groove und Flow des Satzes einen maximalen Gegensatz bildet zum zupackenden Hauptthema, das sogleich wieder einsetzen wird und ganz auf die Betonung schwerer Schlagzeiten hin konzentriert ist. Was die Leichtigkeit der vorangegangenen Episode im Rückblick nur nochmals betonen wird. 


\section{Frank Bridge (1879-1941): Klavierquintett d-Moll H. 49a (1907/1912)}

Das Klavierquintett von Frank Bridge existiert in zwei deutlich voneinander verschiedenen Fassungen. ${ }^{319}$ 1904/05 verfasst und hiernach mehrfach aufgeführt, legte der Komponist es beiseite und unterzog es schließlich 1912 einer grundlegenden Revision. Am 29. Mai 1912 wurde diese Version uraufgeführt. Ebenfalls in London in der Royal College of Music Union, wo schon die Premiere der Erstfassung am 28. Mai 1907 gegeben wurde. Fast genau fünf Jahre zuvor. Deutlichsten Ausdruck fand diese Überarbeitung in der Verschmelzung von Adagio und Scherzo zu einem neuen, gemeinsamen Mittelsatz. Aber auch die anderen beiden Sätze wurden partiell revidiert. Der leise Beginn des Eröffnungssatzes im Unisono von erster Violine und Cello etwa kam neu hinzu. Es ist diese zweite Fassung, über die ich hier spreche.

Und der neue Anfang ist wichtig für jene Passage, die etwa drei Minuten später erklingen wird (Satz I, Ziffer 4, T. 72-83, ca. 2.47-3.12 min). Dem neuen, nun sehr zarten Beginn folgt Allegro moderato ein lang angelegter Spannungsaufbau, der seinen Höhepunkt in einer fortissimo zu spielenden Doppeldominante (E7/9) von d-Moll, der Haupttonart des Satzes, in T. 58 (2.16 min) findet. Dieser Höhepunkt mäandert in der Folge aus, gleich den schwächer werdenden Wellen, die ein ins Wasser geworfener Stein provoziert. Das Tempo der harmonischen Progression wird verlangsamt (T. 58: E7/9, T. 62: g, T. 64: c7/b5). Das Register des vollen Streichquartettsatzes sinkt graduell Schritt für Schritt ab. Mit dem zweigestrichenen fis der ersten Violine und eingestrichenem fis des Cellos am Höhepunkt in T. 58 gestartet, finden sich die beiden Außenstimmen der Streichersatz 10 Takte später fast zwei Oktaven tiefer (g bzw. c) wieder. Ab hier lichtet sich auch die Dichte des Streichersatzes, während im Klaviersatz die kreisenden Akkordarpeggien übergehen in eine viertaktige, aufsteigende, vier Oktaven durchschreitende Akkordbrechung der in T. 68 erreichten neuen Dominante C7/9 - mit dem F-Dur-Dreiklang als neuer Tonika, der Dur-Parallele des bisherigen d-Moll des Satzes, wird in T. 72 jene Passage einsetzen, die hier interessiert. Der ganze Abschnitt ab T. 58 ist sehr delikat. Chromatik wird betont (vgl. die Außenstimmen fis - f, ges - g). Aber die Wirkung ist eher die von matten Pastellfarben, keine von scharfkantiger Dissonanz. Der Taktwechsel 67/68 steht stellvertretend für diese Klangcha- 
rakteristik, wenn sich das es und ges des c7/b5 in das e und g der C7/9Dominante wandelt. Die daran anschließende F-Dur-Passage, um die es mir hier geht, wird also mit graduellen Schattierungsänderungen vorbereitet, die insgesamt den Effekt haben, als ob die Energie der Musik langsam abbremst. Obwohl das, was nun ab T. 72 als Seitenthema folgt, gleichfalls von Chromatik durchzogen sein wird (vgl. nur ab T. 79 über dem Orgelpunkt $\mathrm{g}$ die Durakkordfolge $\mathrm{C}-\mathrm{H}-\mathrm{B}-\mathrm{A}-\mathrm{As}-\mathrm{G}$ ), ist seinem Anfang ein signifikanter Aufhellungseffekt zu eigen. Die C7/9-Dominante hatte ihn bereits vorbereitet. Das reine F-Dur, mit dem das Klavier anhebt, und der plötzliche Wegfall des Streichsatzes, der bis hierhin dominiert hatte, wirken dennoch wie das Heraustreten auf eine Lichtung. Aber nicht in gleißendes Licht. Eher wie ein Hinausluschern auf den verbleibenden Halbschatten.

Dieser ganze erste Satz von Bridges einzigem Klavierquintett ist leidenschaftlich. Aber er ist dabei elegant, anmutig. Und in keinem Moment mehr als hier, im dolce piano con pedale des Klaviers ab T. 72ff.: Eine Musik, die Leichtfüßigkeit im Satz und Leidenschaftlichkeit in der Melodiebildung zu verbinden vermag. Dabei bleibt die klangliche Aussage mehr Ahnung als beherztes Statement. Die Betonung der ersten Schlagzeit ist ausschließlich in die hohe Lage der Melodie verlegt. Im 6/8-Metrum wird im Übrigen die schwere Schlagzeit des ersten Achtels ausgespart. Erst jeweils auf der schwachen zweiten Schlagzeit wird zu begleitenden Akkordbrechungen angesetzt. Ergänzt um eingestreute Quartolen und gegen Ende übergebundene Vorhalte, wirkt das Ganze eher ephemer, wie kaum zu greifen. Wenn man sich orientiert hat, ist der Augenblick schon vorbei.

Diese Musik kommt wieder, zuerst im vollen Ensemblespiel und später, ab Abschnitt 17, nochmal unverändert, aber nun in D-Dur und wieder vom Klavier alleine. Hier wirkt die Passage im Vergleich viel resignierter, nach dem, was musikalisch in der Zwischenzeit geschehen ist, aber auch aufgrund des eine kleine Terz tieferen, deutlich dumpferen Registers des Klaviers. Ein eindrucksvolles Beispiel dafür, wie sehr Lage zählt für die Wirkung.

\section{Florent Schmitt (1870-1958): \\ Klavierquintett h-Moll op. 51 (1909)}

Schmitts Werk wurde am 27. März 1909 in der Pariser Société Nationale de Musique uraufgeführt. ${ }^{320}$ Wir haben es hier mit den Proportionen und Aus- 
drucksextremen einer Mahlerschen Sinfonie zu tun. Fast eine Stunde Aufführungsdauer. Jene Stelle, die mir in der für mich trotz aller thematischen Kohärenz beim Hören kaum überschaubaren Fülle an formalen Dispositionen, emotionalen Zuständen, harmonischen Brüchen und satztechnischen Strategien immer ins Ohr springt, erscheint dabei fast genau zur Mitte von Partitur und Referenzaufnahme (Satz II, ca. 9.03-10.03 min). Und es ist ein Moment von ausgenommener Transparenz und Delikatesse, Schmitts Lehrer Gabriel Fauré würdig, dem dieses Werk gewidmet ist.

6 Takte vor Ziffer 15 beginnt sich der Streichersatz plötzlich aufzulösen. Liegetöne. Eine nach der anderen Stimme steigt aus. Darunter durchschreitet das Klavier, über weite Strecken in unisono geführten Oktaven, einen Tonraum von drei Oktaven nach unten. Es spinnt dabei thematisches Material weiter, das unmittelbar zuvor im vollen Ensemble ausgeleuchtet worden war. Wie ein Nachhall. Mit dieser Passage wird der Musik nicht nur ein Gros ihrer zuvor überbordenden, in konzertanter Manier inszenierten Energie entzogen, sondern auch ein Tonartwechsel vorbereitet. Aus dem Bereich von E-Dur mit einer Betonung des gis-Moll-Klangs auf der III. Stufe kommend, erreicht man mit Ende dieser Überleitung auf T. 1 von Ziffer 15 C-Dur. Sechsmal wird hier nun vom Klavier in unterschiedlichen Lagen der C-Dur-Tonikadreiklang harfengleich in Zweiunddreißigsteln in ansteigenden Umkehrungen gebrochen. Über den liegenden C-Dur-Akkord der Streicher in konstant tiefer Lage. Nun folgt der Hauptteil des Abschnitts: Eine Harmonie pro Takt. C-Dur - g-Moll - C-Dur - g-Moll C-Dur - g-Moll. Mit drei Akkordbrechungen je Takt in wechselnden Umkehrungen im Klavier. Mit einer gleichmäßig voranschreitenden melodischen Linie, die sich aus dem jeweils tiefsten Ton der Akkordbrechung, sprich aus der Folge der Umkehrungen ableitet. Beim dritten Mal C-Dur greift schließlich die 1. Violine diese melodische Binnenlinie in Variation auf. Dann kippt alles in den Beginn der Passage zurück: Die einleitende absteigende Basslinie im Klavier kehrt wieder, nun mit halbem Umfang. Doch die Funktion ist dieselbe. Ein Tonartwechsel wird vorbereitet, nun nach Es-Dur. Dies wird mit Ziffer 16 erreicht. Es wird sodann fast dasselbe noch einmal gespielt, nun in höherer Lage und um eine Wiederholung verkürzt, bevor mit einer letzten, nun nur noch zweitaktigen Basslinie ab Ziffer 17 die Streicher zu übernehmen beginnen. Und die Musik in eine andere Richtung weiterzuziehen beginnt. 
Die Akkordfolge, die das Herzstück dieses Abschnitts grundiert, ist eigentlich schlicht. Sie lebt davon, dass der neu etablierten Tonika ihre Durdominante vorenthalten wird. Anstelle derer erscheint die Mollvariante. Die melodische Binnenlinie der Akkordbrechungen betont dies, indem sie von der Terz e (bzw. g) zur Mollterz b (bzw. des) führt. Die Geschwindigkeit der Akkordbrechungen tut das ihre. Die Situation hat es ungemein Erhabenes für mich. Aber in einer ausnehmend eleganten Spielart. Unaufdringlich. Nicht der große Pinselstrich, das weite Panorama. Nur eine Nuance. Und eine im Kontext der Kunstfertigkeit dieses Klavierquintetts vergleichsweise einfache Stelle. Aber gerade dies Lichte, das dieser Augenblick in die Partitur einbringt, verfehlt nie seine Wirkung auf mich. Im Übrigen in allen Aufnahmen, die ich kenne, so klar hebt sich dieser Moment aus der Partitur ab. ${ }^{321}$

\section{Paul Le Flem (1881-1984): Klavierquintett e-Moll (1910)}

Gebrochene e-Moll-Akkorde des Klaviers in hoher Lage eröffnen Paul Le Flems Klavierquintett, das am 19. März 1910 in der Société National de Musique in Paris uraufgeführt wurde. Sie plätschern. Wie Nieselregen. Instabil dahinmäandernd in ihrem Gegensatz von binärer und tertiärer Metrik in linker und rechter Hand, im Kontrast des Brummelns des tiefen e in Kontraoktavlage und des Glitzerns der Arpeggien in viergestrichener Oktavlage meilenweit darüber. Größtmöglicher Tonraum, maximale Weite. Die Atmosphäre ist gesetzt. Bretagne. Meer. Herbst. Grau. Man kann sich schwer von der Meerassoziation befreien. Von der Farbe und Bewegung der Musik her. Aufgrund ihrer ganzen geradezu kinematographisch-atmosphärischen Landschaftsinszenierung. Das Tempo des Anfangs ist von Le Flem besonders langsam gewählt: Viertel in 48, Lent. Die Streicher setzen in T. 3 im Unisono ein. Leise. Die Musik lässt sich Zeit. Das Tempo der harmonischen Progression ist gleichfalls langsam.

Die ersten 18 Takte klingen hierdurch wie ein Vorspann. Die Szenerie eröffnet mit einer Landschaft noch ganz ohne Protagonisten. In T. 19 nimmt die Musik dann plötzlich Fahrt auf (ca. 1.30-1.51 min). Immer noch zart. Pianissimo. Aber schneller. Der Satz des Klaviers wechselt von Achteln in Zweiunddreißigstelakkordbrechungen. Die beiden Violinen in Achteltriolen. Das Cello spielt im Pizzicato Grundton und Quintette jenes Ak- 
kords, der die ganze Passage T. 19 bis 24 prägt, welche das Ende der Einleitung markiert: a-Moll. Mit dem Auftakt zu T. 25 setzt dann das erste, beherzt vorgetragene Statement des Satzes ein. Davor? Alles Atmosphäre. A-Moll nicht als Dreiklang. Sondern impressionistisch ausgemalt, koloriert mit Quarten, Septimen und Nonen, die unaufgelöst für sich stehen. Letztlich wird das a-Moll nur durch die Basspartie im Cello (T. 19-22, 24) bzw. der linken Hand des Klaviers (T. 23) als solches markiert. Kaum mehr als angedeutet. Im Übrigen brechen Violinen und Klavier in ihrer Begleitung einen e-Moll-Septakkord. Und auch die Melodie der Bratsche ist aus diesem Tonvorrat gestaltet. Das Flirrende der Passage wird hierdurch befördert, dass sich nämlich Subdominante und Tonika in e-Moll quasi überlagern.

Der Höhepunkt dieses Abschnitts ist in T. 23 erreicht, wenn die Melodie der Bratsche, die in T. 20 eingesetzt hatte, ihren Zielton g als Septime des a-Moll-Akkords erreicht und zugleich das Cello vom Pizzicato auf der ersten Schlagzeit coll'arco urplötzlich ins gestrichene Spiel zurückwechselt. Die Cellolinie schreitet in gleichmäßigen Vierteln voran: fis $-\mathrm{e}-\mathrm{d}-\mathrm{h}$. Gefolgt von einem Sprung zurück in die dann in Takt 24 gehaltene Grundquinte a - e. Dass das fis - e im Cello nicht nur wie ein Stoßseufzer, sondern wie ein sich auflösender Sekundvorhalt zum Grundton e klingt, illustriert trefflich das Moment der Überlagerung von Subdominante (a-Moll) und Tonika (e-Moll), die diesen Abschnitt prägt. Der Moment besonderer Poesie ist hier nun die Kollision des g der Bratsche und des Sextvorhalts fis - e im Cello in hoher Lage auf Schlagzeit 1 von T. 23. Diese Reibung verschwindet so rasch wie sie gekommen war. Doch die ganzen anderthalb Minuten Musik bis hierin fühlen sich plötzlich an wie ein Hinarbeiten auf diesen einen ephemeren Augenblick. Wie sich Licht mitunter im rauen Meer bricht, wenn Lichteinfall und Interferenzen verschiedener Wellen für einen kurzen Moment eine Reflexion provozieren wie ein Glitzern, das so schnell vergeht wie es erscheint und mit dem man nicht gerechnet hat, es zu sehen.

\section{Sergej Tanejew (1856-1915): Klavierquintett g-Moll op. 30 (1911)}

Leises steht hier neben Lautem, Zartes neben Brachialem, liedhaft Einfaches neben kontrapunktisch Komplexem, Kammermusikalisches neben 
Konzertant-Orchestralem, mehrstimmig ausdifferenzierter Satz neben solchem, in dem die fünf Instrument vollständig zu einer Einheit verschmelzen. Tanejews spätes Klavierquintett ist eine Kathedrale. Und neben dem Klavierquintett op. 18 von Mieczyslaw Weinberg (1945) jener Beitrag zum Genre mit der größten Renaissance im Tonträgermarkt und in den Konzertprogrammen zuletzt.

In exemplarischer Weise ausgestaltet ist diese Spezifik des Denkens in Extremen im Finale: Allegro vivace in einer Passage etwa gegen Mitte des Satzes, zwischen den Ziffern 223 und 227. Wenn Tanejew auf kurzem Raum eine lang vorbereitete große konzertante Geste samt vollgriffiger Akkordsprünge des Klaviers in wenigen Takten in einen fast schon impressionistischen, von Trillern durchzogenen Streichersatz überführt, der dann in eine Passage mündet, welche maximale Zartheit ausdrückt. Diese acht Takte der Ziffer 226 sind die lyrische Variante desjenigen Themas, welches in unterschiedlichsten Gestalten quasi als Idée fixe bereits vielfach in den gut 40 vorangegangenen Minuten Aufführungsdauer zuvor angeklungen war. Beginnend mit seinem prägnanten melodischen Kopfmotiv im Rahmen des Seitenthemas des ersten Satzes Allegro patetico (vgl. Satz I, Ziffern 21, 25). Diese Passage des Finales ist ein Beispiel dafür, dass in diesem Werk viele thematische und motivische Ideen immer wieder aufgegriffen, variiert und schließlich im Finale zusammengeführt werden, um das Werk in seinen enormen Dimensionen zusammenhalten. Das ist aber nicht der Grund, warum ich immer wieder zu ihr zurückkehre. Die viertaktige Trillerpassage Ziffer 225 hat gerade erst in Quart-Sext-Stellung die nun folgende neue Tonart G-Dur etabliert und mit ihrer Diatonik das Klangbild gelichtet, wenn mit Ziffer 226 ein Moment von seltener Schönheit erscheint (ca. 5.39-5.55 min). Ein Augenblick, der ganz auf einer D-Dur-Dominante ruht, deren Quart-Sept-Gerüst $\mathrm{d}-\mathrm{c}-\mathrm{g}$ auf Liegentönen von Cello und Bratsche die ganze Passage überspannt. Das Klavier steuert gleichmäßige Achtelbrechungen bei, die allerlei diatonische Vorhalte einstreuen. Es ist jedoch die einsam darüber thronende, zuvor bereits gehörte, nun aber voll ausgeschriebene viertaktige melodische Linie der ersten Geige, die den Unterschied macht. Die mit einmaliger variierter Wiederholung über die acht Takte hinweg den Tonraum von fast zwei Oktaven durchschreitet und eine erhabene Sehnsucht ausstrahlt. Musik ist eine Kontextkunst. Doch selten so deutlich wie hier, wo sich dieser kleine Moment erst im Licht der 
Kathedrale, die ihn musikalisch umgibt, in seiner Schlichtheit zu voller Größe entfaltet.

\section{Jean Huré (1877-1930): Klavierquintett D-Dur (1912)}

Jean Huré gehört zu einer goldenen Generation bretonischer Komponisten um Louis Aubert (*1877), Paul Ladmirault (*1877), Jean Cras (*1879), Rhené-Baton $(* 1879)$, Paul Le Flem $(* 1881)$ und dem etwas älteren Joseph-Guy Ropartz (*1864), die Kammermusik von einer großen ästhetischen Affinität zueinander hinterlassen haben. Gerade im Bereich der Klavierkammermusik, wie stellvertretend die in diesem Buch aufgegriffenen Klavierquintette von Cras, Huré und Le Flem veranschaulichen. Avancierte Tonalität reich gerade an unaufgelösten Quart-, Sext-, Sept- und Nonakkorden und aufwändig gebrochenem, oft virtuosem Klaviersatz prägen das Klangbild, ohne impressionistisch im Sinne der historisch dominierenden Zeitgenossen Claude Debussy und Maurice Ravel zu klingen. Spröder im Charakter ist das Schaffen dieser Gruppe. Eher Schwarz-Weiß-Photographie als extrovertiert farbengesättigtes Gemälde. Aber stets konturscharf. Und reich an Momenten von großer Intensität.

Die Postludio überschriebene Schlusspartie von Hurés gut halbstündigem, einsätzigem Klavierquintett, uraufgeführt am 23. Mai 1912 in Paris, hat einen solchen Moment (ab Ziffer 47 bis Takt 9 nach Ziffer 49, ca. 26.42-28.30 min). Andante ist das Tempo, D-Dur die Tonart - so wie sie bald 27 Minuten zuvor mit Beginn des Stücks etabliert wurde. Die Tonart wird hier in zunächst einfachster Diatonik wieder frisch gesetzt. Pianopianissimo. Die Bratsche stimmt ein gleichmäßig in Halben und Vierteln voranschreitendes Thema an, das bis hierhin schon vielfach zu hören war. Aber nie in dieser klaren, einfachen, offen vor einem stehenden Liedform. Korrespondierend gleichmäßig die Begleitung des Klaviers, in sachten Akkordbrechungen. Es sind diese Schlichtheit und Gleichmäßigkeit, die im scharfen Kontrast stehen zur der dekomponierten Passage zuvor (Ziffern 45 und 46, ab ca. $24.36 \mathrm{~min}$ ), die einen langen, sehr leidenschaftlichen Abschnitt (ab Ziffer 35, ca. 20.24 min, zunächst eher schroff, ab Ziffer 40, ca. 22.35 min dann deutlich lyrischer, aber mit gleichbleibend hoher Energie) abbremst und nach Orientierung sucht. Die Musik operiert mit zuvor bereits bekanntem Motivmaterial. Das Ensemble bricht jedoch als Einheit immer 
mehr auf, geht in ein Wechselspiel Streicher - Klavier über, bevor nur noch das Klavier bleibt. Das nach Kontur fahndet und mehrere vage Ansläufe mit eben jenem Quartsprung unternimmt, der ab Ziffer 47 dann in der Bratsche das Hauptthema eröffnen und als melodisches Intervall den ganzen Schlussteil überformen und prägen wird. All das führt jedoch zunächst zu nichts. Bis plötzlich, Ziffer 47, das besagte Hauptthema in seinem ganzen liedhaften Glanz einsetzt. Das vom Klavier begleitete Bratschensolo währt am Ende nur 5 Takte. Sein Effekt ist jedoch groß. Die Musik gewinnt schlagartig an Fasslichkeit und Zug, nachdem Huré ihr zuvor alle Energie genommen hatte. Einmal etabliert, treten bis Ziffer 48 zunächst nach und nach die verbleibenden drei Streicher hinzu. In der Folge wird immer mehr an Intensität aufgebaut, die Lautstärke erhöht, die Harmonik verdichtet, bis schließlich der Höhepunkt im Übergang von T. 7 zu T. 8 nach Ziffer 49 erreicht wird, bevor die Musik Schritt für Schritt in ihren langsamen Schluss überführt wird und ausläuft. Welch erhabener Moment ist dieser Höhepunkt stets für mich! Die 1. Violine bindet an ihrem höchsten Punkt auf zweigestrichenem d über, bevor der besagte Quartsprung folgt. Der Beginn der melodischen Linie des Hauptthemas ist so nach vorne verlängert. Das erhöht die Intensität des Intervalls. Der Quartsprung wirkt dadurch wie eine Auflösung. Befördert dadurch, dass auf T. 8 die Dominante erreicht wird und das liegende $\mathrm{d}$ der 1. Violine zum Quartvorhalt innerhalb des Dominantakkords umgedeutet erscheint. Es ist ein kurzer Augenblick nur. Aber wie durch einen Spalt öffnet sich plötzlich eine endlose Weite vor einem. Wie, wenn man einen historischen Turm besteigt, eine lange Strecke über dunkle Stufen hinter sich bringt, oben heraustritt und das erste Mal in die Ferne schauen kann. Dabei ist es der skizzierte weite Weg an Musik zuvor, der erst den außerordentlichen Effekt dieses flüchtigen Moments im Übergang von T. 7 zu T. 8 nach Ziffer 49 ermöglicht. Eine beeindruckende dramaturgische Anlage in ihrer Spannweite.

\section{Georgy Catoire (1861-1926): Klavierquintett g-Moll op. 28 (1914)}

Im Fall von Georgy Catoires Klavierquintett op. 28 ist es der Schluss des Finals (ab Ziffer 60 bis zum Ende, ca. 6.21-7.11 min). Das dreisätzige Stück ist wohl 1914 entstanden. Für April 1915 sind jedenfalls russische Rezensionen von Aufführungen zu greifen. ${ }^{322}$ Und die Veröffentlichung der 
Noten folgte 1921 in Moskau. Die Referenzaufnahme, eingespielt 2007, scheint freilich jenseits von Rundfunkübertragungen die Erstaufnahme zu sein. Mit Ziffer 60 endet ein ziemlich turbulentes, zuletzt ausnehmend dramatisches Finale, das über die Ziffern 55-58 eine geradezu konzertante Inszenierung gesehen hatte. Virtuos gerade für den Pianisten. Deren Intensität über Ziffer 59 hinweg allerdings in Lage und Lautstärke Stück für Stück zurückgenommen wird bis zur leeren, tiefen Quinte der Durtonika GDur auf Schlagzeit 1 von Ziffer 60. Die ganze Passage hiernach changiert mit den harmonischen Grundfunktionen über den immer präsenten Tonikagrundton g hinweg. Entsprechend groß ist der Effekt, wenn die Subdomaninantparallele über g für einen kurzen Moment die Quint von e nach es eingetrübt bekommt, 5 Takte vor Ende.

Es ist jedoch das dreifache rasche Echo von 2. Violine, Bratsche und dann 1. Violine über der Subdomaninantparallele a7/g auf die Eröffnungslinie der 1. Violine ab T. 4 nach Ziffer 60, der meine ganze Aufmerksamkeit gehört. Zusammen mit dem Lagenwechsel der Violinen, den fortwährenden Vorbehaltbildungen, den Akkordtupfern und -brechungen des Klaviers sowie der beständigen Reibung mit dem stets präsenten Tonikagrundton entsteht ein feines, ganz filigranes, nach Auflösung sehnendes Gespinst. Es ist so schnell vorbei wie es gekommen ist. Und doch steht man da und fragt sich, was einem da gerade widerfahren ist. Wie Moment wie eine Epiphanie.

\section{Ignacy Friedman (1882-1948): Klavierquintett C-Moll (1918)}

In der ersten Hälfte des 20. Jahrhunderts zählte Ignacy Friedman zu den angesehensten Pianisten weltweit. ${ }^{323}$ Insbesondere als Chopin-Interpret. Ein Ruhm, der nur unvollkommen über Tonaufnahmen konserviert ist. Sein Komponieren hingegen ist Insiderwissen. Wie das des Dirigenten Bruno Walter, dessen Klavierquintett sich ebenfalls in der Ausstellung findet.

Doch was für eine herrlich morbide Klanglandschaft betritt man nach 12 Minuten des zweiten Satzes von Friedmans c-Moll-Klavierquintett! Nichts bis dahin hat einen darauf vorbereitet. Die Musik unmittelbar zuvor gibt vielmehr einen treffenden Eindruck von der Ausdrucksvielfalt des Satzes. Ab T. 17 nach Buchstabe C (ca. 6.16 min) war ein schneidig akzentuiertes Menuett mit deutlichem Walzeranklang im 3/4-Takt erklungen. 
T. 17 nach Buchstabe D (ca. 7.37 min) ging dieses über in eine elegante Barkarole im 9/8-Takt, die zwischen Dur und Moll hin und her flackert. Ab T. 18 nach Buchstabe E (ca. 9.25 min) folgte, Vivo con leggerezza, im 3/2Takt ein Marsch. Sehr aufgeregte Musik. Mit einem auffallend akzentuierten Sechstonmotiv im Bass $(\mathrm{d}-\mathrm{cis}-\mathrm{f}-\mathrm{d}-\mathrm{c}-\mathrm{d})$, das trefflich in John Williams' Soundtracks zu Krieg der Sterne gepasst hätte. Mit diesem Motiv hatte bereits gut 10 Minuten zuvor der Satz begonnen.

Was die vorgenannten Aggregatzustände in ihrem steten Wechsel bereits andeuten: Es handelt sich bei diesem Larghetto um einen Variationensatz. Bei dem besagtes Sechstonmotiv eine prägende Rolle spielt. Bis zum Schluss. Der nun, nach Minuten konstant hochgehaltener, aber fortwährend variierter Intensität mit einem plötzlichen Bruch beginnt. T. 15 nach Buchstabe F (ca. 10.19 min). Radikal dünnt das Satzbild aus. Zunächst bleibt nur ein fis liegen (Klavier), dann ein c (2. Violine) und schließlich ein d (Bratsche). Über diesen leeren Klang hallt eine kurze Sechszehntelwechselfigur mehrfach nach. Bis nur noch der Ton d liegt. Andante molto serioso hebt in g-Moll eine einstimmige Linie im Klavier an, die Schritt für Schritt mit schließlich beißender Chromatik bis zur Siebenstimmigkeit verdichtet wird. Die ganze Passage hat das Flair einer Fuge, die T. 8 nach Buchstabe G in einer Dominante endet, die auf Schlag 1 mit c, cis und es als Dissonanzen beginnt und sich binnen eines Taktes zum reinen D-Dur lichtet, bevor das ganze Ensemble in ein unisono gespieltes g kippt.

Hier nun beginnt nach gut 12 Minuten (T. 399-441, ca. 12.00-14.58 min) jene morbide Klanglandschaft, mit der der Satz beschlossen wird. Eine Oktavbrechnung wird eingeführt, in gleichmäßigen Achteln, die das Klavier fast bis zum Ende dieser Schlusspassage spielt. Der erreichte Grundton wird, erst in 2. Violine, dann in der Bratsche, von einer kleinen, triolischen Sechszehntelfigur umspielt. Sie kommt auch hiernach mehrfach wieder. Aufbauend auf Tonfolge und Intervallverhältnis des Hauptthemas, nun nur diatonisch gesetzt, klingt diese Figur eher wie ein Gitarrenriff. Sie hat etwas Schattenhaftes in ihrer Flüchtigkeit. T. 15 nach Buchstabe G erscheint in der 2. Violine das Hauptthema nochmals in ganzer Breite in seiner eigentlichen Form. Dabei ist es gerade der chromatische Eingangsschritt dieses Hauptthemas, der in dieser Schlussvariation abgespalten und zum eigenständigen Stilmittel emanzipiert wird. Oft in scharfen Kontrast zu den Liegetönen bzw. Oktavbrechungen anderer Stimmen gestellt. Diese dissonanten Farben in Verbindung mit dem rhythmisch gleichmäßigen 
Voranschreiten, den vielen Liegetönen/Oktavbrechungen und der chromatischen Aufladung der hier durchschrittenen Mollsphäre sind es, die das Morbide der Stimmung hervorrufen. Und Friedman lässt sich hierfür Zeit. 1/6 der gesamten Aufführungsdauer des Satzes wird auf diesen Abschnitt verwandt. Das ist wohl proportioniert. Denn diese sehr spezielle Atmosphäre, die hier erzeugt wird, braucht Zeit, um ihre Wirkung zu entfalten.

\section{Gabriel Pierné (1863-1937): Klavierquintett e-Moll op. 41 (1919)}

Der II. Satz in Gabriel Piernés Klavierquintett, am 22. Februar 1919 in der Société National de Musique in Paris uraufgeführt, ist ein spanischer Tanz. Genauer genommen ein baskischer. Ein Zortzico. Aber eher die Erinnerung von Parisern an eine Reise in den Süden als Aufenthalt dort. Mehr ihr Berichten gegenüber Freunden daheim, während sie versuchen, ihnen jene Tanzschritte beizubringen, die sie bei Gelegenheit dort unten gelernt haben. Und sich zurücksehnen, weil sich ihr Körper durch die Bewegungen erinnert. Wie es war. Wie es sich angefühlt hat, dort zu sein. Impressionistische Musik. Zart und warm. Schwerelos. Verspielt. Ein kontinuierliches Drehen und Wehen. Und doch von einem dezenten Hauch Melancholie durchzogen. Wie wenn man realisiert, dass etwas nicht mehr ist. Dass es sich nur noch wie eine Erinnerung anfühlt. Federleicht. Wie ein Strich über die Wange. Und doch wie ein leichtes Seufzen im Lächeln. Eine Musik, die zupackt, aber immer etwas unsicher bleibt in ihrem 5/8-Metrum. Immer etwas haltlos.

Wenn etwa zu Anfang von Ziffer 31 (ca. 1.22-1.32 min) die äußeren Streicher und das Klavier mit klaren Ansagen in Des-Dur den schlichten Wechsel von Dominate zu Tonika konturieren, die beide je vier Takte füllen. 2. Violine und Bratsche aber Chromatik hineinschummeln. Wie die Tonfolge in der 2. Violine über die vier Tonikatakte: as - a $|\mathrm{c}-\mathrm{ces}| \mathrm{b}-$ bes $\mid$ as. Keine beißenden Dissonanzen. Ganz zart. Versteckt in der Mittellage. Kaschiert zudem über große Lagensprünge. Aber die ungetrübte Heiterkeit der diatonischen Melodie der 1. Violine verändernd sich hierdurch. Weil nicht selige Terzen oder Sexten entstehen. Sondern Intervalle wie Tritoni. Kleines Detail, großer Unterschied. 


\section{Arnold Bax (1883-1953): Klavierquintett g-Moll GP 167 (1920)}

Autobiographische Deutungen haben (fast) immer etwas Spekulatives. ${ }^{324}$ Oft etwas Anstrengendes, da Apologetisches. Sie stehen allzu leicht anstelle einer eigenen Meinung, dem Zulassen einer eigenen Interpretation. Und spätestens seit dem berühmten Aufsatz Der intentionale Fehlschluss (1946) von Monroe C. Beardsley und William K. Wimsatt vertritt in der Ästhetik kaum einer mehr ernsthaft, dass es auch nur darauf ankäme für die Beurteilung eines Werks, Beweggründe oder Absichten seines Schöpfers zu kennen: Einmal in der Welt, wird die jeweilige Musik unabhängig. Doch auch wenn Beardsley und Wimsatt über Literatur schrieben, hat mir ihre Position für die narrativ ungleich abstraktere Musik immer erst recht eingeleuchtet. Doch bilden die Intensität, Wucht und oft diabolische Freude in Bax Klavierquintett eine Herausforderung für diesen modernen ästhetischen Glaubenssatz. Es entstand während der Zeit des Ersten Weltkriegs, den Bax, aufgrund einer Herzerkrankung ausgemustert, von fern beobachtete. Viele seiner Freunde jedoch dienten und starben an der Westfront oder in Übersee. Mehr noch war Bax mit seiner Familie nach Dublin übergesiedelt. Und engagierte sich überdies persönlich für die republikanische Sache in jenem polarisierten politischen Klima, das 1916 zum gescheiterten Osteraufstand und wenig später in den Irischen Unabhängigkeits- und dann Bürgerkrieg mündete. Wäre dem nicht genug, war Bax privat zur selben Zeit in eine Affäre mit der Pianistin Harriet Cohen verstrickt, für die er wenig später Frau und Kinder verlassen sollte. Es scheint in jener Phase der Entstehung seines Klavierquintetts keinen Aspekt in Bax Leben zu geben, der nicht maximal aufgewühlt und unausgeglichen war. Und sein Klavierquintett klingt jedenfalls genau so.

Es war wie viele Werke jener Jahre von Bax für Cohen komponiert, die gemeinsam mit dem English String Quartet am 19. Dezember 1917 in London auch seine private Erstaufführung gab. Die offizielle Premiere folgte in Londons Queen's Hall am 12. Mai 1920. Dieses Klavierquintett ist reich an emotionalen Vortragsbezeichnungen. Es gibt in ihm Momente von schroffer Gewalt und solche von morbider Resignation. Augenblicke überbordender Freunde und solche ekstatischer Leidenschaft. Szenen melancholischen Sehnens und solche unverhohlener Bedrohung. Aber Bax bezeichnete sich zeitlebens als überzeugten Romantiker. Und es sind die mephisto- 
phelischen Episoden, in denen ich diese Charakteristik bei Bax am deutlichsten höre. Kein Novalischen Hymnen an die Nacht, keine Friedrichschen Allegorien. Aber teuflisch-verspielt im Sinne E.T.A. Hoffmanns, fast schon boshaft im Geiste Heinrich Heines.

Die Passage ab T. 7 nach Buchstabe B mit Auftakt im Finale des Klavierquintetts ist eine solche Stelle (T. 59-75, ca. 2.25-3.05 min). Musik, die später im Satz noch ein zweites Mal wiederkehren wird. Das volle Ensemble ist im Einsatz. Der Komponist verlangt rhythmisch betontes und expressives Spiel. Der formale Aufbau des Abschnitts ist einfach. Periodenbau nach Lehrbuch. Zweitaktiger Vordersatz des Klaviers, zweitaktige Nachsatzantwort der 1. Violine. Der Rest begleitet. Das Spiel wird wiederholt, mit alternativem Nachsatz. Dann übernimmt die Bratsche den Vordersatz. Das Klavier antwortet. Eine Antwort, die beim zweiten Mal von den anderen Streichern übernommen und fortgeführt wird in eine kleine Überleitung des Streichquartetts. 6 Takte, bis der nächste Formteil anhebt. Die jeweils zweitaktige harmonische Abfolge in G-Dur läuft über den durchgehend präsenten Tonikagrundton $\mathrm{g}$, spielt mit dem steten Wechsel von Dur und Moll in den Akkorden der Kadenzstufen und erlaubt hierdurch einiges an Farbigkeit in der motivisch stabil gehaltenen, repetitiv angelegten Melodieführung: C6/g [S] - D7 $7-3 / g$ [D] - C6/g [S] - g6 [t] D G - d7 $7^{\mathrm{b}} 5 / \mathrm{g}$ d6 $5 / \mathrm{g}$ $\mathrm{d} 7 / \mathrm{g} \mathrm{D} 4 / 9 / \mathrm{g}[\mathrm{d} / \mathrm{D}]-\mathrm{g}^{0}-\mathrm{d} 7^{\mathrm{b}} 5 / \mathrm{g}$ d6 $5 / \mathrm{g} \mathrm{D} 4 / 7 / \mathrm{g}$ D7/g - D7/g. Was diese Akkordsymbole nur unvollkommen andeuten können, ist das hohe, delikat umgesetzte Tempo der chromatischen Schattierungswechsel. Sein volles Potential wird jedoch unüberhörbar mit dem Einsatz des Themas in der Bratsche erreicht (T. 67f.). Durch die zweifache Präsentation dieser melodischen Phrase zuvor im Klavier vorbereitet, ist dieser an sich schon sehr prägnante Vordersatz zu diesem Zeitpunkt für den Hörer melodisch bereits klar etabliert. Das macht sich Bax zunutze, um in der Bratschenvariante nun jenes $\mathrm{f}$ zu inszenieren, das an dieser Stelle der entscheidende Ton für den Charakter der Musik ist, indem es die hier eigentlich erreichte Dominante D nach Moll verschiebt. Die Eleganz der melodischen Phrase profitiert dabei, jedenfalls für meine Ohren, sehr davon, wenn das Tempo wie in der Referenzaufnahme etwas zurückgenommen und die Passage nicht wie in den anderen Vergleichsaufnahmen zu hören fast gehetzt gespielt wird. ${ }^{325}$ »Cantabile. Singing«, wie es in der Partitur heißt. Denn dann erst gewinnt dieser Moment für mich jene Doppelbödigkeit, die ein mephistophelisches 
Grinsen ausmacht. Es ist beeindruckend, was für einen beachtlichen Unterschied für die Wirkung an dieser Stelle alleine die Wahl des Tempos macht.

\section{Gabriel Fauré (1845-1924): \\ Klavierquintett Nr. 2 c-Moll op. 115 (1921)}

Es ist für mich das eindringlichste Unisono der Geschichte dieses Genres. ${ }^{326}$ Takt 4 nach Ziffer 4 im III. Satz. Bei 3.21 min. Andante moderato. Der Aufbau bis dahin hat enorme Ausmaße. Und doch sind es nur 17 Takte. Er beginnt ab Ziffer 3 (ca. 2.03 min). Klavier solo im Wechsel mit unisono geführten langsamen Streicherlinien der oberen drei Streicher. Das Klavier etabliert eine synkopische Begleitung. Die Musik schwebt. Alles ist leicht. Und doch schwermütig. Graduell wird die Intensität angehoben, bis bei ca. 2.50 min (T. 10 nach Ziffer 3) das ganze Ensemble wieder zusammentritt. Der Streichersatz wird hier wieder vierstimmig. Er verdichtet sich aber zugleich durch hinzutretende Chromatik und rhythmische Versetzung der Stimmen. Und gewinnt Schritt für Schritt über eine Oktave an Tonraum.

Das besagte Unisono auf dem Ton $\mathrm{h}$ ist das Ziel dieser ganzen Entwicklung. Der Höhepunkt. Es spannt sich über zwei Oktaven auf. Es markiert einen Endpunkt. Und zugleich den Beginn einer hinreißenden achttaktigen Partie in Sequenzen, die erst an Energie gewinnt, bevor sie schrittweise in sich zusammenfällt, um mit Ziffer 5 auf der Tonika G-Dur zu enden. Das Herzstück des Satzes. Hiernach setzt die Musik neu an. In der Aufnahme des Quintetto Fauré di Roma klingt dieser Moment, wenn der Ton h erreicht ist, wie ein Stoßseufzer. Alles löst sich auf. Und doch ist nichts geklärt.

Faurés zweites Klavierquintett hatte am 21. Mai 1921 Premiere. Wie so viele bleibende Stücke französischer Kammermusik in der Société Nationale de Musique in Paris. Doch Fauré, zu diesem Zeitpunkt schon von einem massiven Hörleiten geplagt, konnte das Feine und das Verwundete dieser Musik zu diesem Zeitpunkt selbst schon nicht mehr hören.

\section{Alfred Schnittke (1934-1998): Klavierquintett op. 108 (1976)}

Es liegt nahe, Alfred Schnittkes Klavierquintett als Repräsentanten musikalischer Postmoderne zu rezipieren. ${ }^{327}$ Dazu trägt schon die zeitliche Nähe 
zur Diskussion um die sogenannte Polystilistik bei, die untrennbar mit dem Namen Schnittke verbunden ist und ab 1968 in seinem Denken und Wirken Raum greift. Es geht weniger um Zitate und Allusionen. Sondern um ein Nebeneinander ganz unterschiedlicher Musiktraditionen. Das wird in dem Klavierquintett von Schnittke auch für den Laien leicht nachvollziehbar ausgeführt. Anstatt etwa auf erst zu identifizierende Zitate aus Werken Dritter, wie sie in postmoderner Musik jener Zeit oft so wichtig sind, greift Schnittke auf Klassiker musikgeschichtlicher Referenzialität zurück: Im 2. Satz wird - wie in vielen Kompositionen Schnittkes - das B-A-C-H-Motiv prominent ausgelotet. Des Weiteren begegnet man im Klavierquintett dem spätgregorianischen Dies-Irae-Motiv in Gestalt seiner ersten Töne, die sich im zweiten Satz schrittweise mit dem B-A-C-H-Motiv verschränken (vgl. dort T. 197ff.). Auch das gleich zu Beginn des Klavierquintetts exponierte Kreuzmotiv cis $-\mathrm{d}-$ cis - his - cis findet sich mit derselben, eine Todesthematik akustisch symbolisierenden Funktion schon bei Bach. So entstehen letztlich Bezüge zu zahlreichen, ganz unterschiedlichen musikalischen Epochen und Ausdrucksformen, u.a. mittels barocker Seufzer-Figuren, klassischem Sonatenhauptsatz und eben der in der Rezeption fest mit musikalischer Romantik verbundenen Gattung des Klavierquintetts selbst. Geradezu als Musterbeispiele für jene Polystilistik können dabei die ersten beiden Sätze dieses fünfsätzigen Werks gelten:

Die Bitonalität des Beginns des ersten Satzes inszeniert Polystilistik z.B. durch das Übereinanderlagern von an sich zwar einfach identifizierbaren, da klar konturierten musikalischen Elementen. Die im Kontext der musikalischen Konventionen aber, als ungleichzeitig klassifiziert, eben nicht gleichzeitig erwartet werden. Dieser Prozess, dessen Ansatzpunkt an dieser Stelle zunächst auf harmonischer Ebene inszeniert wird, vollzieht sich in einer anhand der Noten auffällig plakativen Weise: Verschiedene Tonarten werden bei deutlichem Lagenabstand auf die beiden Hände des Pianisten verteilt (T. 1-2: cis-Moll über C-Dur, T. 3-4: G-Dur über g-Moll, T. 5-6: Des-Dur respektive Cis-Dur über D-Dur).

Im zweiten Satz verlagert sich die Idee der Polystilistik auf die Ebenen von Stilelementen und Epochenkennzeichen. Es trifft ein strukturell recht überschaubar gehaltener g-Moll-Walzer von Beginn an auf das B-A-C-HMotiv, wenig später dann gleichzeitig auf das spätgregorianische DiesIrae-Motiv. Insgesamt ist der Satz mit der Anhäufung an Sekundreibungen überdies von unverkennbar zeitgenössischem Charakter. Nicht zuletzt 
provoziert durch das fortwährende Aufeinandertreffen des Tones $\mathrm{h}$ des B-A-C-H-Motivs mit der Mollterz b des g-Moll-Tonikaakkordes. Vier verschiedene musikalische Zeitalter und Milieus sind so in verschiedenen Schichten der Musik des zweiten Satzes präsent und überlagern einander: Spätmittelalterliche Gregorianik (Dies-Irae-Motiv), Barock (B-A-C-HMotiv), großstädtische Unterhaltungsmusik des 19. Jahrhunderts (Walzer) und Neue Musik des frühen 20. Jahrhunderts (Exponierung nicht tonal begründeter Sekundreibungen).

Dergleichen bietet dem Interpreten Ansatzpunkte für die Interpretation und dem Hörer Ebenen, die er verfolgen kann. Dies ist sehr klug konstruierte Musik. Doch scheint mir das nicht der entscheidende Grund dafür zu sein, dass sich in diesem Fall die Diskographie geradezu überbordend ausnimmt. Zwei Dutzend Aufnahmen! Schnittkes Klavierquintett ist tonträgerseitig der größte Hit des Genres post-1945, wenn es denn in der abseitigen musikalischen Nische der Kammermusik etwas wie einen Hit geben kann. Und das mit einem durchaus sperrigen, anstrengenden, in vielem intellektuellen Stück. Die gleichzeitige, unmittelbar emotionale Kraft dieser Musik jedoch mag hier eine entscheidende Rolle dafür spielen, dass ausgerechnet dieses Werk so viele Künstler interessiert. Mein Zugang erfolgt jedenfalls primär über diese Ebene, auch wenn mir die Polystilistik dieser Musik auch intellektuell viel Spaß bereitet. Doch dieses Stück ist vom Komponisten zuvorderst als Trauermusik angelegt. Am stärksten vielleicht im Finale. Wenn die anmutige Schlichtheit einer vierzehn Mal in hoher Lage wiederholten Klaviermelodie auf eine ausgesprochen dissonante, mit Vierteltonreibungen arbeitende Streicherfaktur in deutlich abgesetzter, tiefer Lage trifft. Das Werk ist Schnittkes verstorbener Mutter zugeeignet und greift immer wieder ihre Vorlieben auf. Der Charakter der Gedenkkomposition reicht bis zur zirkulären Anlage des Klavierquintetts. Diese geht soweit, dass das Werk an seinem Ende einen unmittelbaren Anschluss an den Beginn erlaubt. Man könnte es dadurch theoretisch immer wieder in ununterbrochener Folge wiederholen. Und dies mit Tonaufnahmen auch tatsächlich unschwer ausprobieren. Mit beeindruckender Wirkung. Dieser konzeptuelle Effekt, der in der Praxis von Aufführungen und Tonaufnahmen natürlich nie umgesetzt wird, ist stärker als jener irgendeiner Einzelstelle dieses Stücks auf mich: Eine faszinierende Idee für ein Werk über Tod und Ende. 


\section{Thomas Adès (*1971): \\ Klavierquintett op. 20 (2001)}

Aufschlussreich ist die Aufstellung der Aufführungen auf der Website des Komponisten Thomas Adès. ${ }^{328}$ Seit der Uraufführung am 29. Oktober 2001 beim Melbourne International Festival of the Arts sind 78 Darbietungen verzeichnet und zwei weitere für 2019 angekündigt. ${ }^{329}$ Die Präsenz ist über die Jahre ziemlich gleichmäßig. Die Gruppe der Interpreten vergrößert sich stetig. Die Liste durchzugehen, gibt einem einen exemplarischen Eindruck, was es heißt, unter den Bedingungen zeitgenössischer Kammermusik ein Erfolgsstück, ein regelmäßig aufgeführtes Werk zu sein. Das ist interessant, denn Adès' Musik ist trotz seiner Prominenz unter den zeitgenössischen Komponisten durchaus widerständig, beißend, schräg, kompliziert. Diese Musik hat nichts mit Kuschelklassik, Filmmusik oder Neoklassik zu tun. Sie ist anstrengend. Fordernd. Und sucht Extreme. Man vergleiche nur das Spektrum der Dynamiken in der Partitur. Oder die Heterogenität, ja das scheinbare Wirrwarr der metrischen Angaben in den Noten. Oder die Komplexität der rhythmischen Relation der Instrumente. Aber wie andere heutige Komponisten auch, scheut Adès nicht mehr Tonalität um jeden Preis. Sie wird vielmehr als Kontrastmittel immer wieder zugelassen. Und macht diese Musik dadurch anschlussfähig, zugänglich, nahbar.

Markierungen in der Partitur deuten darauf hin, dass man Adès Klavierquintett als dreisätzige Komposition lesen kann (Satz I: Ziffern 1-12, II: 13-18, III: 19-24). Auch wenn diese Musik von gut 20 Minuten Aufführungsdauer ohne Pause gespielt wird und ihre zwei Hauptthemen alle Werkteile hindurch präsent bleiben. Die mit dem Komponisten am Klavier entstandenen Tonaufnahmen weisen jedenfalls eine Dreiteiligkeit über unterschiedliche Tracknummern als solche aus. Folgt man dieser Strukturannahme, findet sich die Passage, die mich stets besonders interessiert, am Ende des zweiten Teils. Die Partitur, die auf der Website des Verlages zur Gänze einzusehen ist, weist dieser Partie die Ziffer 18 zu (ca. 3.58-5.05 min). Sie folgt einem langen Abschnitt 17, zu dessen Beginn das Klavier zunächst auf Einstimmigkeit reduziert und dann ganz aus dem Ensemblespiel genommen wird. Die Streicher erkunden für den Rest von Ziffer 17 im homophonen Satz unterschiedlicher Sonoritäten, ausführlichen Vorgaben hierzu in der Partitur folgend. Die Musik verlangsamt sich und wird leise. Es ist alles präzise ausnotiert, klingt aber wie ein lang angelegtes 
Rubato. Mit Ziffer 18 erscheint plötzlich das Klavier wieder. Eine Variante des zweiten Themas. Die Streicher setzen in tiefer Lage ihr Spiel in Klangblöcken fort, nur um gelegentliche kleine motivische Akzente erweitert und metrisch gegeneinander verschoben, so dass ihre Begleitung etwas Waberndes bekommt. Das Klavier operiert gleichfalls mit homophonen Akkorden in langsamem Wechsel, aber in höherer Lage und metrisch unabhängig von den Streichern. Das sieht man in der Partitur schon auf den ersten Blick daran, dass hier die Taktstriche von Streichquartett und Klavier versetzt zueinander liegen. Eine darüber vermerkte, alternative rhythmische Notation der Klavierstimme verdeutlicht den unbedingten Willen zur Unabhängigkeit der beiden Ensembleteile nur noch mehr. So wirkt die Klavierstimme an dieser Stelle einerseits kristallklar und präzise, andererseits ein wenig desorientiert. Korrespondierend muten die Dissonanzen in den Klavierakkorden durch die weite Lage des Akkordsatzes fast schon sanft an. Dies ist Musik, die aufwendig zyklisch konzipiert ist und detailreich notiert. An der Stelle mit der stärksten Wirkung auf mich jedoch vor allem atmosphärisch wirkt und einen stark improvisatorischen Gestus hat, obwohl die Partitur keinen Aufwand scheut, Präzision zu fordern und ihr Erreichen durch aufwändige Umschreibung zu fördern. Dieser Kontrast zwischen Machart und Effekt und der desorientierte Charakter der Passage machen ihn für mich aus.

\section{Antón García Abril (*1933):}

\section{Alba de Los Caminos (2007)}

Uraufgeführt wurde Alba de Los Caminos am 22. Juli 2007 in Málaga beim Festival de Música Clásica de Villanueva del Rosario. Antón García Abril war zu diesem Zeitpunkt bereits Mitte $70 .{ }^{330}$ Nach einer langen Laufbahn als Komponist und vor allem als Musikpädagoge an Spaniens höchsten Ausbildungsstätten. Überraschenderweise hat Alba de Los Caminos alles, was die so jung auftretende Neoklassik will. Das Jetzige. Das Zugängliche. Das Repetitive. Das Tonale. Das Popwissen im Klassikgewand. Diese Musik holt einen ab. Aber sie nimmt einen eben auch mit. Auf etwas, dass die Reise lohnt. Wo etwas passiert. Das Zuhören einfordert. Das anspruchsvoll zu spielen ist. Und hierdurch der Musik umgekehrt mehr ermöglicht. 
Die ohne Pause gespielten fünf Sätze sind verschieden im Ausdruck, reich an Farben, Kontrasten und Melodien. In ihrem thematischen Material und ihrer formalen und thematisch-motivischen Disposition sind die Werkteile dabei aufwändig zyklisch miteinander verbunden. Der Charakter ist freilich oft rhapsodisch, wirkt frei wie eine Fantasie. Aber das Stück hat Momente von enormer rhythmischer Energie und Zielstrebigkeit. Ein solcher erscheint mit Beginn des zweiten Satzes (vgl. T. 1-15, ca. 0.00-0.20 min). Wie aus dem Nichts. Mit dem Gestus einer großen Rockband. Die Akkordfolge dieser Passage in Des-Dur über den Orgelpunkt As könnte auch von Gruppen wie The Who stammen. Offen. Monumental. Erhaben. Assus4 - As7sus4 - Desj7/As - Beses/as [notiert als A/gis]. Volles Ensemble. Forte. Schnell: Allegro brioso. Im Rhythmus scharf betont und irregulär. Dreitaktige Phrasen. Der Puls wechselt im 2/2-Metrum stetig zwischen 4/4, 3/4 und 2/4, immer wieder zusätzlich versetzt mit der Betonung schwacher Schlagzeiten. Aber doch ist die Musik regulär und symmetrisch genug, um so etwas wie Groove und Flow zu entwickeln. Die tiefen Streicher spielen einen harten, gleichmäßigen Beat. Die Musik treibt so mächtig voran. Klassik mit Punkenergie. Ohne Crossover zu sein. Es ist diese Energie, auf die ich stets höre, die mich in diese musikalische Situation hineinzieht.

\section{Albin Fries (Gerhard SchlüssImayr) (*1955): Klavierquintett Nr. 1 h-Moll op. 10 (2007)}

Albin Fries ist das Pseudonym, unter dem Gerhard Schlüsslmayr seit gut anderthalb Jahrzehnten im spätromantischen Stil komponiert. Beruflich ist er Konzertpianist und seit 1985 als Solokorrepetitor an der Wiener Staatsoper tätig, seit 1989 als stellvertretender Studienleiter. Zeitgemäß habe er komponiert, erklärte er mir in einem eMail-Gespräch, bis ein persönliches Erlebnis, eine Geschenkkomposition und deren kühle Aufnahme, in ihm auslöste, für das Ganze nochmals im spätromantischen Stil anzusetzen. Und diese Erfahrung wie eine Befreiung gewirkt habe und eine enorme Schaffensfreude in ihm auslöste. Es sagt viel über das heutige Musikleben im Bereich Klassischer Musik, das ein Künstler von dieser Qualität und diesem professionellen Background es für notwendig erachtet, für ein derartiges Projekt in Retromanier doch besser einen Künstlernamen zu wählen, so provozierend wirkt es auf viele. Die meisten von Fries' Werken sind in 
Eigenregie in Tonaufnahmen und Noten über IMSLP zugänglich gemacht und zugleich über einen eigenen Kanal auf YouTube. Ein Musterbeispiel dafür, was das Internet für eine letztlich Nischenkultur wie das Klavierquintett an Gewinn gebracht hat. Insbesondere für quer liegende Sonderfälle wie diesen. Man muss nach wie vor gefunden werden. Interpreten gewinnen. Sich ein Publikum schaffen. Die Herausforderung bleibt. Wächst eher noch in der Angebotsflut. Aber man kann gefunden werden. Ungleich einfacher als früher. Wie im Fall von Fries mit ein paar Klicks.

Fries hat zahllose Lieder geschrieben, Opern, Klaviermusik. Aber es sind die großdimensionierten Kammermusikwerke, denen meine unverhohlene Zuneigung gehört. Den beiden Klavierquintetten, den zwei Streichquartetten und dem Klavierquartett. Schlicht weil ich sie oft höre. Immer wieder hören mag. In dem, was sie sein wollen, sind sie für mich vollkommene Musik. Sie sind überreich an musikalischen Situationen, die ich hier unter dem Stichwort »emotional impact« ausstellen könnte. Eine typische findet sich im langsamen II. Satz des ersten Klavierquintetts in h-Moll. T. 75-89 (ca. 5.05-6.08 min). Mit dieser Passage kommt ein ziemlich aufgewühlter Abschnitt des Satzes zur Ruhe. E-Dur. Piano. Das Klavier eröffnet solo. Fragiler Satz: Triolen über oft übergebundenen Duolen. Ein feingesponnenes Netz. Alles Nuance. So viel passiert gar nicht. Stufenharmonik. Gleichmäßige Melodieführung mit einem wirkungsvollen, aber doch dezenten kleinen Akzent am Phrasenende.

Es ist nicht einfach, auf einen Blick zu erklären, warum diese Musik nicht trivial klingt. Aber sie tut es nicht. Sie ist anmutig. Sie ist retro, aber nie Fake. Das ist kein postmodernes Referenzspiel. Keine Ironie. Keine Dekonstruktion. Diese Musik will so sein. Sie klingt dabei stets aufrichtig empfunden. Sie ist ernst gemeint. Im Kontext populärer Musik würde man an dieser Stelle authentisch sagen. Es war eine Entdeckung für mich, ästhetisch zu erfahren, dass diese Zuschreibung auch unter den Bedingungen Klassischer Musik, wo ich so gänzlich anders sozialisiert bin in meiner Erwartungshaltung, genauso für mich funktionieren kann wie wenn etwa ein Singer-Songwriter mit einem Lied, das auf denselben Akkorden und Tonfolgen basiert wie schon zahllose vor ihm, mich wirklich berühren kann, weil es glaubhaft mir etwas über den Künstler erzählt. Oder durch die Art seines Tuns über mich. Und Kategorien wie Fortschritt oder Innovation in diesem Augenblick nebensächlich werden. Das Gleiche ist eben nicht dasselbe. 


\section{Vijay lyer (*1971): Time, Place, Action (2014)}

Vijay Iyer, mittlerweile Professor in Harvard und MacArthur Foundation Fellow, ist einer der bekannten zeitgenössischen, seit Mitte der 2000er Jahre besonders viel diskutierten Jazzpianisten. ${ }^{331}$ Wie viele andere der renommierten Klavierspieler dieser Profession seiner Generation auch, wie etwa Brad Mehldau (*1970), Jason Moran (*1975) oder Robert Glasper (*1978), erkundet Iyer die Grenzen des Jazz zu anderen Musikbereichen und verlässt neben dem Kerngeschäft der Solo- und Ensemblearbeit, die klar im Jazzkontext verortet, mit Jazzmusikern produziert und als Jazz ausgewiesen ist, diesen immer wieder auch ausdrücklich. ${ }^{332}$ Time, Place, Action gehört zu eben diesen Aktivitäten. ${ }^{333}$ Uraufgeführt am 15. Februar 2014 in Houston, ist dies schon das zweite Werk Iyers für Klavierquintett. Nach Mutations I-X (2005), einer zehnteiligen Suite für Klavierquintett und Elektronik. ${ }^{334}$ Time, Place, Action ist dem afroamerikanischen Schriftsteller und Politaktivisten Amiri Baraka gewidmet. Es ist bislang nicht auf Tonträger veröffentlicht. Nach Fries schon das zweite jener Werke in dieser Ausstellung, für die man hoffen muss, in den Tiefen des Internets fündig zu werden.

Die Sache liegt hier aber noch komplizierter. Denn Time, Place, Action changiert in seinen acht Sätzen zwischen notierter Komposition und improvisierten Partien. Es entstand für das Brentano Quartet, das mit Iyer u.a. auch die Uraufführung spielte. ${ }^{335}$ In einem längeren Videointerview auf dem YouTube-Kanal von Ensemblemitglied Mark Steinberg gibt Iyer ausführlich Auskunft über sein Konzept für Time, Place, Action. Er spricht vor allem über den Fokus des Stücks auf Fragen von Timing und Groove, wenn man gleichzeitig das Tempo moduliert, kombiniert mit den besonderen Herausforderungen für die Ensembleinteraktion in einem teilimprovisierten Kontext. ${ }^{336}$ Was intellektuell und abstrakt klingt. Aber einen stark unmittelbaren Effekt hat in den Händen eines Improvisationskünstlers wie Iyer. Gleich drei unterschiedliche Livemitschnitte in derselben Besetzung sind derzeit im Internet verfügbar, die einen Eindruck zu geben vermögen von dem Spielraum, den die Partitur gewährt. Mit deutlich unterschiedlichem Ergebnis.

Meine Referenzaufnahme ist eine Version aus dem April 2014. Sie ist die in meinem Ordner. Eine Übertragung durch WQYR, New Yorks Radio- 
station für Klassische Musik. Dort ist es der dritte Formteil, der gespielt wird (ca. 4.32-8.10 min). Nach kurzer Einleitung und mehreren Anläufen mündet die Musik in eine ostinate Figur des Streichquartetts, eine Art stilisierter Tango, über die das Klavier improvisiert. In dieser Interpretation verdichtet sich der Klavierpart ab ca. 7 min zunehmend und geht über in eine ungemein elegante Repetition von leisen homophonen Klavierakkorden (ca. 7.11-7.32 min). In der Version von 2017 spielt Iyer im entsprechenden Werkteil (ca. 34.28-37.50 min), der dort erst als vierter Formteil und fünf Minuten später erscheint, etwas gänzlich anderes. Die für mich besondere musikalische Situation ist in diesem Fall also gar nicht festgelegter Teil des Werkes, sondern Augenblick in einer bestimmten Interpretation und dem dortigen Erkunden des Improvisationsraums geschuldet, den die Partitur gewährt. Ein Grenzfall also, inwiefern man hiermit noch etwas über das Stück selbst sagt. Denn das, was mir an der Referenzaufnahme so besonders hörenswert erscheint, ist Teil des Werks - schon aufgrund der Streichquartettpartie - und doch zugleich nicht, da nicht regelmäßig zu hörender Bestandteil dieses Klavierquintetts.

\section{Bent Sørensen (*1958): \\ Rosenbad - Papillons (2014)}

Zachary Woolfe verglich 2017 in der New York Times die Musik von Bent Sørensen mit der Malerei von George Seurat. ${ }^{337}$ Die Referenz zum großen französischen Pointillisten der Fin de Siècle und der Tradition in der Malerei, die Seurat mitbegründete, finden sich immer wieder in Berichten über Sørensens Musik. So schief Vergleiche zwischen den Künsten, Epochen und Stilen stets bleiben müssen: In diesem Fall kann man auf diesem Weg tatsächlich in plastischer Weise eine irritierende, aber charakteristische Eigenschaft von Sørensens Musik überraschend präzise veranschaulichen, die einem auch in vielen Gemälden des Pointillismus begegnet. Diese Bilder zeigen einerseits klar konturierte Figuren und Gegenstände bei streng geometrisch durchkomponiertem Bildaufbau, andererseits verschwimmen ihre Konturen durch die Malweise sofort und dies je stärker, je näher man herantritt. Dabei schwirren sie geradezu, bedingt durch die Arbeit mit kleinsten Farbtupfern und dem Simultankontrast von benachbarten, nebeneinanderliegenden Farbflächen. 
Genau solche ästhetischen Charakteristika begegnen einem tatsächlich auch in Rosenbad - Papillons wieder. ${ }^{338}$ Uraufgeführt am 28. Februar 2014 in Berlin, keine zwei Wochen nach der Premiere von Iyers Time, Place, Action. Etwa im zweiten Satz Calmo con delicatezza, um den es mir hier geht, eine Musik, die gleichfalls geprägt ist von einem steten Kontrast zwischen Schwirren - hier durch je gut 50 Sekunden lange, atonale Tremolopassagen der Streicher - und Kontur - durch melodisch wie harmonisch klar konturierte Phrasen in romantischer Kadenzharmonik, die in unterschiedlicher Ausführung vier Mal erscheinen (vgl. 0.59-1.06, 1.54-2.08, 2.54-3.08, 3.54-4.50 min). Beim ersten Auftreten wird die Kontur nur angedeutet mittels einer kontextbedingt ungemein poetisch wirkenden melodischen Eröffnungsgeste e - cis - e im Klavier, welche zu einem Tonika-ADur-Akkord gehört und deren Schlusston dann zum Quartvorhalt eines Doppeldominant-H-Dur-Akkordes verwandelt wird. Beim zweiten Mal wird die beim ersten Mal nur angedeutete Passage des Klaviers voll ausgeführt. Beim dritten Mal zunächst von den Tremoli der Streicher überlagert und verschattet, wobei das Tremolospiel dann den Klaviersatz ansteckt und in diesen wandert. Beim vierten und letzten Mal schließlich verleiht eben diese Spieltechnik dem Klaviersatz etwas Stockendes, Stotterndes. Die Kadenzharmonik des Klaviers klingt nun verunsichert inmitten der Streicher, was einen melancholischen Effekt hat.

Die konzeptuelle Nähe zu den ästhetischen Charakteristika, für die der Begriff Pointillismus steht, zeigt sich in diesem Klavierquintett noch auf anderer Ebene: der strengen geometrischen Komposition. Denn RosenbadPapillons gehört zu einer Trilogie: Der Witz der streng geometrischen Komposition ist nicht nur, dass der Klavierpart in allen drei Fassungen der stets siebensätzigen Papillons derselbe bleibt, aber sich die Musik der anderen beteiligten Instrumente und damit der Kontext ändert, in dem der Klavierpart operiert und den mal Streichquartett (Rosenbad), mal Streichorchester (Mignon) und mal gemischtes Streicher-Bläser-Kammerensemble (Pantomine) beisteuern. Die Anordnung der Sätze wechselt überdies je nach Trilogieteil, was ohne eine solch streng geometrische Kompositionsweise nicht sinnvoll möglich wäre: Das Calmo con delicatezza ist mal der zweite (Rosenbad), mal der sechste (Pantomine) und mal der letzte Satz (Mignon).

Doch steht wie im Pointillismus auch die streng geometrische Kompositionsweise gar nicht im Vordergrund des ästhetischen Erfahrungsraums, 
den die Musik hier aufspannt. Ist es dort die spezifische Farbigkeit, konzentrieren sich auch Beschreibungen von Sørensens Musik auf etwas anderes, nämlich atmosphärische Begriffe wie Nebel, Rauch, Echo, Traum, Schattenspiel, Ruhelosigkeit usw., um das Vage, Flüchtige, Flirrende, Präsente und sich doch Entziehende in ihrem Habitus und Timbre einzufangen. Oder auf Begriffe wie Zerbrechlichkeit, Zartheit und Stille, um ihre Intimität zu kennzeichnen. Auch das `Nordische` wird immer wieder bemüht, um sich an ihre räumliche Weite anzunähern. Oder Vergleiche zu Musik von ästhetischen Ahnen wie Claude Debussy, Jean Sibelius und dem Arnold Schöneberg seiner atonalen Phase bemüht, um die harmonische Freiheit und Instabilität von Sørensens Stil anzudeuten. Was all diese Strategien der Annäherung bereits durchblicken lassen, wird beim Hören dieser Musik sofort klar: Sørensen schreibt eine sehr emotionale, einnehmende, unmittelbar zugängliche Musik von hohem Wiedererkennungswert. Viele Kommentatoren reagieren auffallend irritiert über sich selbst, da es ausgerechnet das Wort Schönheit ist, das in ihren Reaktionen auf Sørensens Musik dominiert, aber eben in einem nicht trivialisierend gemeinten Sinn. Eine Selbstverständlichkeit, mit der diese oft tabuisierte ästhetische Kategorie selten im Kontext zeitgenössischer Kunstmusik erscheint. Zu der Sørensen aber unstrittig gezählt und dabei auch stets ihrer avantgardistischen Seite zugeschlagen wird. Alles Schroffe, Brüchige, scharf Akzentuierte, in dynamische und harmonische Extreme Getriebene, sich Entziehende, Flirrende scheint bei Sørensen nie Hauptsache oder gar Selbstzweck zu sein, sondern im Dienste von Momenten von großer Transparenz und Poesie zu stehen. Wie der angesprochenen melodischen Eröffnungsgeste e - cis - e.

\section{Fazil Say $\left({ }^{*} 1970\right)$ : \\ Yürüyen Köşk. Hommage à Atatürk (2017)}

Fazil Says Hommage an den Staatsgründer der türkischen Republik, Mustafa Kemal Atatürk, existiert in vier Fassungen: Klavier solo (op. 72a, UA: 6. September 2017), Klavierquintett (op. 72b, UA: 6. September 2017), Klavier plus Streichorchester (op. 72c, UA: 7. März 2019) und Klavier plus Kammerorchester (auf der Verlagsseite bislang nur als vorliegend angemerkt). ${ }^{339}$ Say tourt derzeit mit der Fassung für Klavierquintett. Auf Tonträger aufgenommen ist bislang aber nur die Version für Klavier solo. ${ }^{340}$ Die Noten der Variante für Klavierquintett sind jedoch bereits erschienen 
und u.a. über verschiedene Bibliotheken unschwer greifbar. Die Verbindung aus Tonaufnahme der Klavierfassung und Partitur gibt genügend Anlass zur Hoffnung.

Says Klavierquintett findet sich also noch gar nicht im besagten Ordner auf meinem iPod. Es steht nur ganz oben auf der Wunschliste nach einer Tonaufnahme. Allein schon der ersten 9 Takte wegen. Diesem eleganten, impressionistischen Changieren auf II. und III. Stufe in C-Dur im 7/8-Takt. Den beiden messerscharfen melodischen Phrasen, die in T. 5 bzw. 7 eingreifen. Der Klangfarben, die das Streichquartett beisteuert, mit Pizzicati, Glissandi und Vogelgeräuschen. All dies einmal zusammen hören zu können. Say steht hier mit Absicht am Schluss. Um eine Grenze zu markieren. An der Neues entsteht. Und Schritt für Schritt erst erreichbar wird für Hörer. Dabei derzeit noch eine Stufe weniger greifbar ist als Iyers Time, Place, Action. Noch einen Schritt vorher liegt. Aber doch schon da ist.

Das Klavierquintett ist ein lebendiges Genre. Ständig kommt neue Musik dazu. Wie zuletzt eben 2007 bzw. 2008 die beiden Klavierquintette h-Moll op. 10 und e-Moll op. 22 von Fries, 2007 Abrils Alba de Los Caminos, 2014 Sørensens Rosenbad-Papillons, Iyers Time, Place, Action oder 2017 eben Says Yürüyen Köşk. Überhaupt sind, seit ich Mitte der 1990er Jahre anfing, mich für das Genre zu interessieren, gar nicht so wenige Werke erschienen, die für mich keine bloße Statistik sind. Keine historiographische Fußnote der Vollständigkeit halber für eine Chronik der Gegenwart des Genres. Oder lediglich intellektuelle Herausforderung, mit der ich mich aus welchem historiographischen Grund auch immer beschäftige. Oder Musik, die mich beeindruckt, ohne aber leider wirklich so richtig meins zu werden. So wie z.B. Olli Mustonens (*1967) dreisätziges Klavierquintett (UA: 12. Juni 2015) mit einem herrlich vorantreibendem, in your face Drammatico e passionato als Eröffnungssatz, das schlicht knallt und keine Gefangenen macht. Düster. Hart. Unerbittlich. Mit Heavy-MetalEnergie. ${ }^{341}$ Sondern viele Arbeiten, die ich tatsächlich immer wieder höre. So wie die in die Ausstellung zu Beginn des Kapitels aufgenommenen Klavierquintette von Lowell Liebermann (1991) und Ian Venables (1995), Philip Gates (1997) und Nikolai Kapustin (1998), Thomas Adès (2001) und Morten Gaathaug (2005), Konstantia Gourzi (2010), Tobias Picker (2011) und Jefferson Friedman (2014).

Fries, Abril, Sørensen, Iyer und Say. Liebermann, Gates, Kapustin, Adès, Gaathaug, Gourzi, Picker und Friedman. Von Spätromantik über Jazz 
und gemäßigte Moderne bis Postmoderne und Avantgarde - das Spektrum, welches das Genre des Klavierquintetts heute erkundet, welches die zuvor genannten Namen stellvertretend repräsentieren, ist enorm. Und reicht bis zu Chilly Gonzales' Neoklassik-Popalbum Chambers (2015). Und das ist nur die Musik, die ich höre. Dabei fehlen in dieser Liste noch all die Namen wirklich harter Avantgardeästhetik, die sich heutzutage gleichfalls immer wieder des Klavierquintetts bedienen. Und die hier nur fehlen, weil dieses Buch keine Geschichte des Klavierquintetts ist, sondern einen Auseinandersetzung mit meinem eigenen Hörverhalten. Mit »emotional impact« auf mich. Und es schlicht unehrlich wäre, fürs akademische Prestige etwa so zu tun, als würde ich diese Musik ebenfalls hören, wenn ich mich nicht beruflich mit dem Genre beschäftige. Was natürlich wenig über die Musik sagt, aber viel über mich und meine Prioritäten als Hörer. Ob es nun die nur Spezialisten vertrauten Protagonisten heutiger Neuer und Neuester Musik sind oder ihre alten großen Namen. Wie Hans Werner Henzes (1926-2012) dreisätziges Quintetto (UA: 25. März 1993) z.B. mit seiner faszinierenden Fassungsgeschichte und dem fulminanten Effekt, wenn es als Schluss in voller Geschwindigkeit einfach abbricht und verhallt. ${ }^{342}$ Oder Elliott Carters (1908-2012) einsätziges, aber in verschiedene Episoden aufgeteiltes Klavierquintett (UA: 18. November 1998), das die Ensembleteile Klavier und Streichquartett in einen scharfen Kontrast zueinander zwingt. ${ }^{343}$ Und eine Grobheit in der klanglichen Inszenierung schafft, die ein geradezu voyeuristisches Interesse weckt. Wie wenn man einem fremden Paar beim Streiten zusieht. Und es auch für den Außenstehenden faszinierend ist, zuzuschauen, gleich wie abstoßend oder absurd oder verstörend der Streit auch wirkt - weil es unübersehbar für die Beteiligten um existentielle Emotionen geht. ${ }^{344}$

Das Klavierquintett ist keine museale, abgeschlossene Praxis. Und mit jedem neuen Beitrag ändert sich das Genre. Und potentiell die >Alternative Musikgeschichte $<$ des besagten Ordners auf meinem iPod. Man hofft darauf. Man tastet sich an neue und neueste Stücke wie Says oder Iyers, Friedmans und Sørensens heran. Man findet erst nur Meldungen von ihnen. Dann Aufführungshinweise. Rezensionen vielleicht. Manchmal Bootlegs auf Plattformen auf YouTube oder als Rundfunkübertragungen. In Ausschnitten oder vollständig. Man beginnt zu ahnen. Und dann kommen die Noten, vielleicht ein Konzert in erreichbarer Entfernung und, mit etwas Glück, am Ende offizielle, hochwertig interpretierte und produzierte Auf- 
nahmen. Die etwas bringen, das einen ein zweites Mal hinhören, zu dieser Musik vielleicht sogar immer wieder zurückkehren lässt.

Es sind gute Zeiten für das Klavierquintett. Und für jeden, der wie ich eine Affinität zu diesem Ensembleformat hat. In der Erschließung von Altem. Aber eben auch in der Schaffung von Neuem. Es gibt so viel zu entdecken. So viel mehr als noch vor kurzem. Und all dies verpasst man, wenn man in dem engen Horizont verharrt, dem einen die Leitmedien Klassischer Musik zugestehen. Zeit, sich auf die Suche zu machen. Und eigene Ausstellungen zu entwerfen. 



\section{Anmerkungen}

1 Vgl. Frédéric Döhl: »Potential und Risiken des Archival Turns in den Digital Humanities für die Musikwissenschaft«, in: Archiv für Musikwissenschaft 75/4 (2018), S. 301-320.

2 In den vergangenen Jahren gab es viel Fundamentalkritik. Vgl. nur für einige bekannte und mit den vielen Reaktionen aus der DH-Community quittierte Beispiele Kathryn Schulz: »The Mechanic Muse: What Is Distant Reading?«, in: The New York Times (24. Juni 2011), www.nytimes.com/2011/06/26/ books/review/the-mechanic-muse-what-is-distant-reading.html; Adam Kirsch: »Technology Is Taking Over English Departments. The False Promise of the Digital Humanities«, in: The New Republic (2. Mai 2014), https://newrepublic. com/article/117428/limits-digital-humanities-adam-kirsch; Danielle Allington/ Sarah Brouillette/David Golumbia: »Neoliberal Tools (and Archives): A Political History of Digital Humanities«, in: Los Angeles Review of Books (1. Mai 2016), lareviewofbooks.org/article/neoliberal-tools-archives-political-historydigital-humanities; Timothy Brennan: »The Digital-Humanities Bust«, in: The Chronicle of Higher Education (15.10.2017), www.chronicle.com/article/ The-Digital-Humanities-Bust/241424; Nan Z. Da: »The Computational Case against Computational Literary Studies«, in: Critical Inquiry 45/3 (2019), S. 601-639, https://www.journals.uchicago.edu/doi/pdfplus/10.1086/702594.

3 Vgl. stellv. Bernhard Rieder/Theo Röhle: »Digital Methods: Five Challenges«, in: Understanding Digital Humanities, hrsg. von David Berry, Palgrave Macmillan: Basingstoke 2012, S. 67-84; Manfred Thaller (Hrsg.): Controversies around the Digital Humanities = Historical Social Research 37/3 [Special Issue] (2012), https://www.gesis.org/hsr/volltext-archiv/2012/373-digital-human ities/; Melissa Terras/Julianne Nyhan/Edward Vanhoutte (Hrsg.): Defining Digital Humanities. A Reader, Ashgate: Farnham 2013; Gerben Zaagsma: »On 
Digital History«, in: BMGN - Low Countries Historical Review 128/4 (2013), https://www.bmgn-lchr.nl/articles/abstract/10.18352/bmgn-lchr.9344/, S. 3-29; Constanze Baum/Thomas Stäcker: »Methoden - Theorien - Projekte«, in: Grenzen und Möglichkeiten der Digital Humanities (= Sonderband der Zeitschrift für digitale Geisteswissenschaften, Bd. 1), hrsg. von dies., 2015, http:// www.zfdg.de/sb001_023; Martin Huber/Sybille Krämer/Claus Pias (Hrsg.): Digitalität. Theorien und Praktiken des Digitalen in den Geisteswissenschaften, 2016, https://digigeist.hypotheses.org/; Mareike König: »Was sind Digital Humanities? Definitionsfragen und Praxisbeispiel aus der Geschichtswissenschaft«, in: Digital Humanities am DHIP, 2016, https://dhdhi.hypotheses.org/ 2642; Mark Sample: »Difficult Thinking about the Digital Humanities«, in: Debates in the Digital Humanities, hrsg. von dies., 2016, http://dhdebates.gc. cuny.edu/debates/text/90; William G. Thomas III: »The Promise of the Digital Humanities and the Contested Nature of Digital Scholarship«, in: A New Companion to Digital Humanities, hrsg. von Susan Schreibman/Ray Siemens/John Unsworth, Wiley Blackwell: Malden/MA 2016, S. 524-537; Jörg Wettlaufer: »Neue Erkenntnisse durch digitalisierte Geschichtswissenschaft(en)? Zur hermeneutischen Reichweite aktueller digitaler Methoden in informationszentrierten Fächern«, in: Zeitschrift für digitale Geisteswissenschaften (2016), http:// zfdg.de/2016_011; Angela Dressen: »Grenzen und Möglichkeiten der digitalen Kunstgeschichte und der Digital Humanities - eine kritische Betrachtung der Methoden«, in: Critical Approaches to Digital Art History = kunsttexte.de Nr. 4, 2017, https://edoc.hu-berlin.de/bitstream/handle/18452/19402/Angela\%20 Dressen\%20-\%20final.pdf; James Smithies: The Digital Humanities and the Digital Modern, Palgrave Macmillan: London 2017; Manfred Thaller: »Geschichte der Digital Humanities« und »Digital Humanities als Wissenschaft«, in: Digital Humanities. Eine Einführung, hrsg. von Fotis Jannidis/Hubertus Kohle/Malte Rehbein, J.B. Metzler: Stuttgart 2017, S. 3-18; Sybille Krämer/ Martin Huber: »Dimensionen Digitaler Geisteswissenschaft. Zur Einführung in diesen Band «, in: Wie Digitalität die Geisteswissenschaften verändert: Neue Forschungsgegenstände und Methoden (= Sonderband der Zeitschrift für digitale Geisteswissenschaften, Bd. 3), hrsg. von dies., 2018, http://www.zfdg. de/sb003_013; Gabriel Viehhauser: »Digital Humanities als Geisteswissenschaften. Zur Auflösung einer Tautologie«, in: Digital Humanities. Perspektiven der Praxis, hrsg. von Peggy Bockwinkel/Beatrice Nickel/Gabriel Viehhauser, Frank \& Timme: Berlin 2018, S. 17-42; Ted Underwood: Distant Ho- 
rizons. Digital Evidence and Literary Change, University of Chicago Press: Chicago 2019.

4 Vgl. zum Begriff Historische Aufführungspraxis Lawrence Dreyfus: »Early Music Defended Against Its Devotees: A Theory of Historical Performance in the Twentieth Century«, in: The Musical Quarterly 69/3 (1983), S. 297-322; Harry Haskell: The Early Music Revival, Thames and Hudson: London 1988; Nicholas Kenyon (Hrsg.): Authenticity and Early Music, Oxford University Press: New York/Oxford 1988; Richard Taruskin: Text and Act: Essays on Music and Performance, Oxford University Press: New York/Oxford 1995; Lydia Goehr: The Imaginary Museum of Musical Works: An Essay in the Philosophy of Music, 2. Auflg., Oxford University Press: New York/Oxford 2007, S. 279-284. Vgl. zum Begriff Invented Tradition Eric Hobsbawm/Terence Ranger (Hrsg.): The Invention of Tradition, Cambridge University Press: Cambridge 1983. Vgl. zum Konflikt zwischen diesen beiden Konzepten im Fall der Barbershop Harmony Frédéric Döhl: »From Harmonic Style to Genre: The Early History (1890s-1940s) of the Uniquely American Musical Term Barbershop«, in: American Music 32/2 (2014), S. 123-171; Frédéric Döhl: »Zu einigen Strategien der Genreetablierung und -verstetigung in einem >Traditionalist Genre` populärer Musik. Die Barbershop Harmony zwischen Historischer Aufführungspraxis und `Invented Tradition««, in: Musiktheorie 30/1 (2015), S. 9-22.

5 Vgl. Wilhelm Altmann: Handbuch für Klavierquintettspieler. Wegweiser durch die Klavierquintette, Verlag für musikalische Kultur und Wissenschaft: Wolfenbüttel 1936; Gottfried Heinz: Die Geschichte des Klavierquintetts von den Anfängen bis Robert Schumann, Männeles: Neckargemünd 2001, S. 237287; Joanne Richardson: The Piano Quintet. Influence of Medium on Genre [Diss.], City University London: London 2014, http://openaccess.city.ac.uk/ 3163/, S. 268-304; Raymond Silvertrust: The Silvertrust Guide to Piano Quintets and Sextets for Piano \& Strings, Eigenverlag: Riverwoods/IL o.J., http:// www.editionsilvertrust.com/guide-to-piano-quintets.htm.

6 Vgl. etwa Carl Dahlhaus: »Zur Theorie musikalischer Gattungen«, in: Systematische Musikwissenschaft, hrsg. von Carl Dahlhaus/Helga de la Motte-Haber, Laaber: Laaber 1982, S. 109-124; Hermann Danuser: »Gattung« in: Die Musik in Geschichte und Gegenwart, 2. Auflg., hrsg. von Ludwig Finscher, Sachteil Bd. 3, Bärenreiter/J.B. Metzler: Kassel/Stuttgart 1995, Sp. 1055-1069; Carl Dahlhaus: »Was ist eine musikalische Gattung?«, in: Carl Dahlhaus. 
Gesammelte Schriften, Bd. 1, hrsg. von Hermann Danuser, Laaber: Laaber 2000, S. 348-358.

7 Vgl. Dick Hebdige: Subcultures: The Meaning of Style, Methuen: London 1979; Sarah Thornton: Club Cultures. Music Media and Subcultural Capital, Polity Press: Cambridge 1995; Ken Gelder (Hrsg.): The Subcultures Reader, 2. Auflg., Routledge: New York 2005; Rupa Huq: Beyond Subculture. Pop, Youth and Identity in a Postcolonial World, Routledge: New York 2006; Ken Gelder: Subcultures: Cultural Histories and Social Practice, Routledge: New York 2007; Ross Haenfler: Subcultures: The Basics, Routledge: New York 2014.

8 Vgl. Will Straw: "Systems of Articulation, Logics of Change: Scenes and Communities in Popular Music«, in: Cultural Studies 5/3 (1991), S. 361-375; Roy Shuker: »scenes«, in: ders., Popular Music: The Key Concepts, 2. Auflg., Routledge: New York 2005, S. 238-240; Robert Futrell/Pete Simi/Simon Gottschalk: »Understanding Music in Movements: The White Power Music Scene«, in: The Sociological Quarterly 47/2 (2006), 275-304; Fabian Holt: Genre in Popular Music, University of Chicago Press: Chicago 2007, S. 6.

9 Vgl. etwa Charles Rosen: The Classical Style: Haydn-Mozart-Beethoven, Faber and Faber: London 1971; Leonard B. Meyer: »Toward a Theory of Style«, in: The Concept of Style, hrsg. von Berel Lang, Cornell University Press: Ithaca/NY 1987, S. 21-71; Leonard B. Meyer: Style and Music. Theory, History and Ideology, University of Pennsylvania Press: Philadelphia/PA 1989; Peter van der Merwe: Origins of the Popular Style: The Antecedents of Twentieth-Century Popular Music, Clarendon Press: Oxford 1989, S. 3; Allan F. Moore: „Categorical Conventions in Music Discourse: Style and Genre«, in: Music \& Letters 82/3 (2001), S. 432-442, hier S. 432; James Wierzbicki (Hrsg.): Music, Sound and Filmmakers: Sonic Style in Cinema, Routledge: New York 2012; Jennifer C. Lena: Banding Together. How Communities Create Genres in Popular Music, Princeton University Press: Princeton/NJ 2012, S. 6.

10 Die nachfolgende Begriffsbestimmung habe ich meinem seit geraumer Zeit im Druck befindlichen Beitrag »Genre« für den Band Musik und Medien entnommen, der im Rahmen der Reihe Kompendien Musik beim Laaber-Verlag erscheinen soll, mit den ausführlichen Literaturangaben zu den Kurzverweisen im Text dann am Ende dieser Endnote.

Es bedarf einer Klarstellung, was ich mit Genre meine, weil Genres unauflöslich immer eine Verbindung von einerseits Kontinuität stiftenden Momenten 
(z.B. musikalische und soziale Konventionen), anderseits Instabilität und hierin doch zugleich eben auch Dynamik verantwortenden Momenten (z.B. Vielfalt der Meinungen und Funktionen) darstellen. Schon auf ganz basaler Ebene macht es einen großen Unterschied, ob man sich für die erstgenannten oder die letztgenannten Aspekte interessiert - oder beides auszubalancieren versucht. Es ist schon deswegen gar nicht so einfach, zu sagen, was ein Genre ist und was es ausmacht, obwohl über Genres zu sprechen Alltag ist. Für Vertreter der erstgenannten Perspektive schaffen Genres vor allem Stabilität im historischen Wandel und Orientierung in einer für den Einzelnen unüberschaubaren Maße an musikalischer Produktion und musikbezogener Kommunikation (vgl. Frith 1996, Negus 1999, Holt 2006, Brackett 2016). Für Vertreter letztgenannter Perspektive erweist sich diese Stabilität als eine leere Behauptung, die im schlimmsten Fall obendrein alles Individuelle dem Diktat von Genrenormen und der Suche nach Ähnlichkeiten und Ähnlichem opfert (vgl. Derrida 1994; Drott 2013). Wieder andere meinen, dass gerade Individualität anders als über vergleichsweise stabile Genrebegriffe gar nicht erst als solche wahrnehm- und beurteilbar wäre (vgl. Carroll 2009).

Welche Position man auch vertritt: Es ist unvermeidbar, dass Musik stets zu Genres gehört, und zugleich unmöglich, Genres quasi rein zu bestimmen (vgl. Berger/Döhl/Morsch 2016, S. 7-10). Genres sind Diskursräume, in denen Schnittmengen ausgehandelt werden (vgl. Atton 2010, S. 523). Immer wird ein Erwartungshorizont an Musik herangetragen, der nicht erst durch diese Musik entstanden ist. Immer verbleiben Dimensionen im Vergleich von Musik und Erwartungshorizont, die nicht zur Deckung gelangen. Man kann keine genrelose Musik schreiben oder spielen - auch wenn Musiker das auffallend oft behaupten, wenn sie für sich künstlerische Autonomie reklamieren (vgl. Toynbee 2000, S. 104; Holt 2006, S. 4; Drott 2013, S. 3). Zugleich kann man keine Musik schreiben oder spielen, in der ein Genre aufgeht - auch wenn Kritiker gerne von >Prototyp< oder >Inbegriff< eines Genres sprechen und damit so tun, als ob eben dies ginge. Das ist, was Jacques Derrida in seinem grundlegenden Aufsatz Das Gesetz der Gattung »Teilhabe ohne Zugehörigkeit« (1994, S. 252) nennt. Schaut man genau hin, sieht man u.a. eine Vielzahl an Akteuren, die sich explizit oder durch ihr Handeln konkludent an Genrebestimmungen beteiligen (1). Man erkennt des Weiteren eine Vielfalt an Funktionen der Genrekategorie (2). Und man beobachtet ein immanentes, fortwährendes, unvermeidliches Ringen von Momenten, die für Stabilität sorgen, mit solchen, die für Instabilität, zugleich aber eben auch für Dynamik verantwortlich sind (3). 
(1) Eine Vielzahl an Akteuren beteiligt sich an der Bestimmung von Genres. Dass es überhaupt solche Akteure gibt, die sich bisweilen sogar gezielt und bewusst um den Prozess der Genrebestimmung bemühen, zeigt sich z.B. immer dann besonders deutlich, wenn Genres gegen die Nutzung durch Dritte verteidigt werden, deren Handeln als genrefremd angesehen wird (vgl. Toynbee 2000 , S. 103, 111). Keineswegs ist dabei gesichert oder vereinheitlicht, wessen Meinung jeweils den Ausschlag gibt (vgl. Brackett 2015, S. 196). Die Erfahrung zeigt allerdings, dass es sehr oft einen großen Unterschied macht, wen man wann in welcher Position fragt (vgl. Anderton 2010, S. 421). Es existieren zudem große Unterschiede zwischen den Genres. Und auch innerhalb eines Genres können sich Sichtweisen ändern (vgl. Lena/Peterson 2008). Die Sache verkompliziert sich dabei nicht nur zusätzlich durch die Vielzahl der Akteure. Akteure können zudem mehrere Positionen gleichzeitig innehaben oder ihre Position wechseln. Ein Labelmitarbeiter kann z.B. gleichzeitig Musiker und Musikhörer sein. Seine Sichtweisen können sich je nach Kontext verschieben, müssen das aber natürlich nicht (vgl. Wald 2009, S. 6). Völlig unabhängig von der Frage multipler Akteurspositionen können sich auch schlicht die Ansichten eines jeden Diskursteilnehmers ändern. Die Gruppen der Akteure sind darüber hinaus in sich heterogen. Musikberichterstattung etwa: Fachmedien werden von professionellen Journalisten betreut, aber z.B. im Bereich der Fanzines von Fans (vgl. Atton 2010). Und verändern ihre Zusammensetzung fortwährend durch Ausscheiden und Neueintritt alter bzw. neuer Mitglieder. Es sind also sehr unterschiedliche, dabei in sich heterogene Typen von Akteuren involviert mit durchaus unterschiedlichen Zielen. Verbände und staatliche Institutionen der direkten Kulturpolitik verstehen sich oft z.B. als Institutionen zur Förderung und zum Erhalt von Genres, wie sich mustergültig an der Verankerung des Jazz im öffentlichen Bildungs- und Subventionssystems studieren lässt (vgl. Ake/Garrett/Goldmark 2012, S. 2). Genredefinition erfolgt dann im Einklang mit diesen Zielen. Industrielle Akteure hingegen orientieren sich z.B. bevorzugt am Ziel der Umsatzgenerierung und -optimierung (vgl. Frith 1996, Negus 1999). Aber auch hier ist Vorsicht geboten. Die typischen Akteure dürfen nicht zu schablonenhaft interpretiert werden: Die Rolle der Musikwirtschaft ist z.B. eine hochkomplexe, die auf sehr unterschiedliche Weise das Feld der Genrebestimmung beeinflusst und in keinem Fall im Sinne binärer Opposition - alles kommerziell Relevante absorbierende, Genreidentitäten dabei negierender Mainstream vs. Genrekulturen (vgl. Miller 2010; Keunen 2014; Nathaus 2015, S. 24f.) - gedacht werden kann. Zumal für alle Ak- 
teurspositionen mehrere Ziele gleichzeitig relevant sein können. Einem Musiker mag es um künstlerische Freiheit gehen und zugleich um soziale Relevanz und hinreichende Einnahmen, um von seiner Musik leben zu können. Die Akteure stehen nicht nur in einem komplexen Verhältnis auf der Ebene der Ziele zueinander. Sie können auch Genres unterschiedlich interpretieren, Fans z.B. die Arbeit eines Musikers genreseitig ganz anders zuordnen als es dem Musiker als adäquat vorschwebt (vgl. Atton 2010, S. 522). Dann steht es dem Musiker frei, das zurückzuweisen. Je nach Ziel (Genrezugehörigkeit, Prestige, Erlöse usw.) mag ihm das aber nichts nutzen. Dabei geht es für die Genrebildung nicht zwingend zuvorderst um Fragen von Konsens und Herrschaft, d.h. darum, wer alles mitreden kann und wer sich am Ende wie durchsetzt. Immer wieder gibt es nämlich auch Genres, für die gerade der Mangel an Konsens und vielmehr der offene Streit über ästhetische und soziale Normen identitätsstiftend ist, gerade in avantgardistischeren Kontexten, die hieraus viel an Dynamik und Produktivität ziehen (vgl. Atton 2012).

(2) Genres dienen überdies ganz unterschiedlichen Funktionen. Die Verwendung von Genrebegriffen ist z.B. eine dominierende Kommunikationsstrategie in der Musikwirtschaft (seit Tonaufnahmen Noten als primäre Distributionsform von Musik abgelöst haben, vgl. Toynbee 2000, S. 103, 115; Wald 2009, S. 89) und in den Kommunikationsmedien, insbesondere im Musikjournalismus. Neben Künstlernamen und Emotionsbegriffen fungieren Genrebegriffe heutzutage mehr denn je als ein Hauptorientierungsmittel in einem für den Einzelnen unüberschaubaren Musikangebot (vgl. Negus 1999, S. 29; Holt 2006, S. 2), das nur in Deutschland alleine im Bereich der Tonaufnahmen alljährlich um mehr als 20.000 Veröffentlichungen wächst und Phänomene wie die Hauptplaylist von Spotify kennt, über die aktuell mehr als 40 Millionen Nutzer wöchentlich personalisierte Empfehlungen bekommen. Dass Genres für Künstler auf Produktionsseite Spielräume und Spielregeln definieren, zu denen sie sich verhalten können, ist jedoch nicht nur in organisatorischer und wirtschaftlicher Hinsicht produktiv. Nach dergleichen Rahmungen und Orientierungspunkten besteht nämlich auch unter den Kreativen selbst eine nicht geringe Sehnsucht in Zeiten, in denen es in den Künsten in vielen Bereichen eben an verbindlichen Grenzen, Dogmatiken und Lehren fehlt, wie schon Adorno konstatierte (vgl. 1970, S. 9), da die alten Maßstäbe ihre einstige Autorität verloren haben (vgl. Kramer 1995, S. 5). Das kann man mit Danto als künstlerische Freiheit feiern (vgl. Danto 1997, S. 5, 12. 27f.). Man kann aber umgekehrt auch den damit einhergehenden Verlust betonen, wie es Lyotard 
tut: »Composers today have the feeling that everything is possible and that they must invent for each work not only its musical form, but the rules of the music« (Lyotard 2009, S. 39). Schon Arnold Schönberg kämpfte mit der Erfahrung, dass ihn die in harmonischer Sicht maximale Freiheit der Atonalität an anderer Stelle massiv einschränkte, da es in einem Gestaltungsraum, in dem alles möglich ist, problematisch wird, zu überraschen oder lange dramatische Formen zu gestalten - und komponierte in Reaktion hierauf ein Jahrzehnt lang nicht. Ihm fehlte plötzlich ein Erwartungshorizont, mit dem er operieren konnte. Genau an einer solchen Stelle machen Genres Angebote. Deren regulatorische Kraft kann man schon daran ablesen, wie wenige erfolgreiche Crossoverkünstler vom Schlage eines Leonard Bernstein, David Bowie, Bobby McFerrin oder André Previn es gibt, die genreseitig ein wirklich weites Betätigungsfeld aufweisen. So steckt in der Anziehungs- und Regulierungskraft des Genrekonzepts für Musikschaffen wie Musikrezeption stets ein ambivalenter Balanceakt zwischen Ermöglichen und Verhindern (vgl. DeNora 2005, S. 148). Aber Genres sind vielmehr. Genres konditionieren in mannigfaltigster Weise den sozialen Umgang mit Musik (vgl. Nathaus 2015, S. 23). Sie fungieren in vielen Fällen z.B. als primärer Bezugspunkt kultureller Identität (vgl. Toynbee 2000, S. 177; DeNora 2007, S. 153; Brackett 2015, S. 190). Genrekonventionen steuern bei allen Akteuren Erwartungen und ermöglichen hierdurch überhaupt erst, zu vergleichen, einen Diskurs zu etablieren und sich nicht in einer disparaten Sammlung von Einzelfällen zu verlieren (vgl. Neale 1980, S. 19; Holt 2006, S. 3; Carroll 2009, S. 93-99). Genres schreiben zudem Musik Bedeutung ein (vgl. Frow 2006, S. 10), stehen aber immer auch zugleich für Wertzuschreibungen Genres sind nie neutrale, bloß sachlich beschreibende Begriffe (vgl. Holt 2006, S. 17). Genrediskurse schließen dadurch eine Vielzahl sozialer Faktoren ein, einschließlich Rasse, Geschlecht, Religion und Nation (vgl. Döhl 2008; Ake/ Garrett/Goldmark 2012, 3f.; Brackett 2015, S. 190; James 2017, S. 24). Genrezuordnungen gehen dabei regelmäßig mit weitreichenden, ganz praktischen Konsequenzen einher, von Qualitätsurteilen und Entscheidungen über Zugehörigkeit bis zur Frage, welche Clubs, Festivals oder Playlists einer Musik offen stehen (vgl. Atton 2010, S. 522; Ake/Garrett/Goldmark 2012, S. 3f.).

(3) Wäre dem nicht schon genug, sind Genres eben stets im selben Atemzug stabil und instabil, gleichermaßen von Kontinuität zu Vorherigem und Veränderungen geprägt (vgl. Wald 2009, S. 28; Atton 2010, S. 522f.; Brackett 2015, S. 190). Es gibt wie gesehen keine Musik, die nicht zu einem Genre gehört, keine Musik, die nur zu einem Genre gehört, und keine Musik, in der ein Gen- 
re aufgeht. Jeder neue Beitrag verändert jedoch zugleich ein Genre und seine Konventionen (vgl. Brackett 2015, S. 195). Es gibt umgekehrt Normen, die übergeordnet in einer Vielzahl Genres auftreten, so die Grundregeln kommerziell relevanter Musik: Einfachheit, Repetitivität und Kürze (vgl. Suisman 2009, S. 277). Dies erschwert gleichfalls die Präzision von Genrebegriffen. Manche Genres werden dabei sehr alt, andere scheinen nur eine Saison, eine Modewelle lang zu existieren (vgl. Berger/Döhl/Morsch 2016, S. 8). Manche Genres bleiben lange allgemein relevant, manche nur kurz, andere wiederum verlassen nie »einen Status des Marginalen und Prekären« (Berger/Döhl/ Morsch 2016, S. 8). Gleichzeitig kann sich ein neues Publikum ein Genre erobern und es dabei auch verändern (vgl. Toynbee 2000, S. 116). Oder der originale Musikerkreis wird verdrängt, etwa im Zuge einer musikindustriellen Erschließung (vgl. ebd.). Musiker fusionieren und erweitern aber auch selbst fortwährend bestehende Genres oder spalten Genrespezifika ab und gewichten sie neu, all dies oftmals sogar gezielt als künstlerische Strategie (vgl. Walser 1993, S. 27).

$\mathrm{Zu}$ diesen drei hier exemplarisch herausgegriffenen Ebenen der Akteure, Funktionen und Dynamiken des Genrebegriffs kommen eine ganze Reihe weiterer grundsätzlicher Herausforderungen hinzu, die Genre zu einer derart fluiden und zugleich attraktiven, da gerade hierin lebensnahen Kategorie machen: So ist der Genrebegriff z.B. nicht trennscharf geschieden von Begriffen wie Stil, Subkultur, Szene oder Gattung. Sie werden mal synonym, mal partiell überlappend, mal kategorisch getrennt voneinander gebraucht. In unterschiedlichen Gegenstandsbereichen westlicher Musik wie klassischer Instrumentalmusik, Oper, Jazz, Filmmusik und Populärer Musik existieren dabei verschiedene Traditionen und Konventionen im Begriffsgebrauch (vgl. Moore 2001, S. 432). Die Lesart dieser Begriffe wechselt aber immer wieder auch innerhalb desselben musikalischen Milieus zwischen verschiedenen Beiträgen. Darüber hinaus treten Unterschiede auf, je nachdem, ob der Beitrag aus der Musikwissenschaft stammt oder aber eine musikalische Praxis zum Gegenstand hat, aber z.B. aus Sicht von Nachbardisziplinen wie der philosophischen Ästhetik, Soziologie, Kulturwissenschaft oder Geschichtswissenschaft verfasst wurde. Neben fragilem Begriffsgebrauch stellt die Quellenlage ein weiteres fundamentales Problem der Genreforschung dar: Regelmäßig fehlt es an klar identifizierbaren, expliziten Gründungsdokumenten, Regelbüchern, Kriterienkatalogen usw., was einen zu einer Diskursanalyse, oft gar Diskursrekonstruktion zwingt, die regelmäßig z.B. Oral History einzubeziehen hat (vgl. Holt 2006, S. 14). 
Schon, ab wann ein Genre existiert und wer für seinen Namen verantwortlich ist (vgl. Fabbri 2012, S. 179f.), lässt sich meist nur ausnahmsweise präzise sagen. Und man muss an dieser Stelle zudem sehr vorsichtig sein, denn allzu oft ergibt eine solche retroaktive Suche vor allem Gründungsmythen und die Behauptung einer quasi reinen Lehre, was das Genre seigentlich` mal war und wieder sein sollte (vgl. Atton 2011, S. 324). Auch wenn die Namensgebung eine hinreichende, aber keine notwendige Bedingung für die Existenz eines Genres ist, so ist die Vergabe von Genrenamen ein wichtiger Indikator, zeigt sich hier doch oft, dass Akteure das Erreichen eines Aggregatzustands ausmachen, dem eine neu gewonnene Spezifik innewohnt, die etwas unterscheidbar macht - worin auch immer diese Eigenart im Einzelfall liegt (vgl. Fabbri 2012, S. 187f.). Ob das Neue dann wirklich neu ist, spielt regelmäßig nur eine untergeordnete Rolle, da es sich bei der Namensgebung zuvorderst um eine soziale, vor allem kommunikative Geste handelt. Im Wechsel und Zusammenspiel der beteiligten Akteursgruppen entstehen fortwährend neue Genrenamen - die dabei keineswegs musikalisch-deskriptiv (Noise Rock, Cool Jazz) angelegt sein müssen, sondern z.B. atmosphärische (Psychedelic, Trance), inhaltlich-thematische (Christian Pop, Christmas Carol), geographische (Charleston, Britpop), sozio-kulturelle (College Rock, Lounge), relationale (Post-Punk, Neo-Soul) oder symbolische (Hair Metal, Baggy) Gesichtspunkte betonen können (vgl. Marino 2012, S. 245f.). Schon in einer eng begrenzten Fallstudie zur elektronischer Tanzmusik im Großbritannien der Jahre 1998 und 1999 förderte Kembrew McLeod mehr als 300 Subgenrenamen unter dem Genremantel der EDM zu Tage, von »abstract beat, abstract drum-n-bass, acid house, acid jazz, acid rave« über »downtempo funk, downtempo future jazz, drill-n-bass, dronecore, drum-n-bass « und »hard chill ambient, intelligent drum-n-bass, intelligent jungle, intelligent techno, miami bass« bis »twilight electronica, two-step, UK acid, UK breakbeat, underground, world-dance« (McLeod 2001, S. 60). Eine zentrale Konsequenz einer solchen Begriffsinflation und -partikularisierung ist natürlich, dass die »normative Bedeutung von Genremodellen und die Halbwertzeit generischer Begriffe« (Wicke 2010, S. 8) sinkt - so wie Genres selbst oftmals nur einen vergleichsweise kurzen Moment im popkulturellen Rampenlicht haben und hiernach vielfach auf deutlich kleinerer Flamme, von einem überschaubaren Kreis von Liebhabern meist dogmatisch deutlich enger ausgelegt (vgl. Lena/Peterson 2008, S. 703-707), weiterköcheln (vgl. Toynbee 2000, S. 116). Das Operieren mit Genrebegriffen ist dabei kommunikativ gesehen in jeder Hinsicht ein permanentes Justieren zwischen zu allgemein (z.B. Pop, 
Jazz) und zu speziell (z.B. Happy Hardcore oder Progressive Low Frequency). Begriffe wie »Metagenre« oder »Subgenre« zeigen dieses Problem an (vgl. McLeod 2001; Anderton 2010). Und doch ist es notwendig, beide Extreme stets gleichzeitig im Blick zu halten - das Verallgemeinern und das Interesse an der Besonderheit des Einzelfalls -, damit die Kategorie des Genres als Verständnis- und Beschreibungsmittel produktiv sein kann (vgl. Holt 2006, S. 7). Genres bedeuten zu einer bestimmten Zeit für bestimmte Leute etwas Bestimmtes (vgl. Brackett 2015, S. 192). Das setzt eine erhebliche historische und soziale Sensibilität voraus, gerade wenn man sich Genres in der Vergangenheit annähert. Denn die Gefahr ist groß, dass man aktuelle Genrevorstellungen auf die Vergangenheit projiziert (vgl. Miller 2010, S. 11). Diese Gefahr ist im hiesigen Fall noch größer als bei anderen historiographischen Fragen, da Genreformierung, insbesondere die damit einhergehende Konventionsbildung, meist stark retrospektive Züge trägt (vgl. Anderton 2010, S. 422). Genres gehen nicht in musikalischen Konventionen auf, sind jedoch keineswegs beliebig in den musikalischen Möglichkeiten, die sie gestatten (vgl. Toynbee 2000, S. 102; Brackett 2016, S. 3). Das Klavierquintett ist hierfür ganz typisch in dem Besetzungsgebot, das es formuliert. Jedes Genre scheint hierbei einen eigenen Toleranzbereich auszuhandeln, bis wohin etwas noch dem Genre als zugehörig angesehen werden kann. Manche sind sehr strikt wie Barbershop Harmony oder Northern Soul (vgl. Toynbee 2000, S. 126; Döhl 2015), andere nicht. Je genauer man die musikalischen Konventionen eines Genres zu beschreiben versucht, desto exklusiver geht man natürlich vor, in dem man zwangsläufig anfängt, Musik auszusortieren, die anderen wie selbstverständlich als dem Genre zugehörig erscheint (vgl. Toynbee 2000, S. 104, 126; Brackett 2016, S. 3). Unterlässt man derart analytische Bemühungen jedoch, ignoriert man die musikalische Praxis, in der regelmäßig ganz bewusst künstlerische Entscheidungen getroffen werden, die z.B. eine Genrezuordnung musikalisch absichern sollen. So problematisch diese widersprüchliche Situation auch bleibt, so klar ist jedoch, dass solche Annäherungen bei aller Vorsicht analytisch durchaus erreichbar sind: Man denke zum Beleg nur an die Möglichkeit, Genres außerhalb ihres Ursprungskontextes zu parodieren (vgl. Brackett 2016, S. 12f). Ohne benenn- und adaptierbare Ähnlichkeiten, die u.a. eine Wiedererkennbarkeit gestatten, wäre dies nicht möglich (vgl. Toynbee 2000, S. 110; Fabbri 2012, S. 186). So oder so muss man jedoch aufpassen, denn das, was in vielen Genres vorkommt, ist nicht zwangsweise weniger wichtig für ein bestimmtes Genre als das, was ihm an Besonderem zu eigen ist und auf das man sich allzu leicht 
konzentriert (vgl. Wald 2014, S. 26). Abgesehen davon, dass gerade musikalische Eigenarten besonders selten Genres exklusiv zu eigen bleiben, sondern gerade und oftmals ausgesprochen rasch ihren Weg auch in andere Genrekontexte finden. Man denke z.B. an Bluesphrasierung oder Funkrhythmisierung. Letztlich muss man Genres relational zueinander verstehen. Genres existieren im Verhältnis zu einem komplexen Netzwerk anderer Genres. Nur ein relationaler Zugriff erlaubt, die Andersartigkeit und zugleich Identität von Genres, nämlich im Verhältnis zu anderen zu verstehen.

In dieser Endnote angeführte bzw. zitierte Literatur: Theodor W. Adorno: Ästhetische Theorie [1970] (= Theodor W. Adorno. Gesammelte Schriften, Bd. 7, hrsg. von Rolf Tiedemann), Suhrkamp: Frankfurt am Main 2003; Jacques Derrida: »Das Gesetz der Gattung« [orig. frz. 1980], in: ders.: Gestade, Passagen: Wien 1994, S. 245-284; Stephen Neale: Genre, British Film Institute: London 1980; Robert Walser: Running with the Devil: Power, Gender, and Madness in Heavy Metal Music, Wesleyan University Press: Middletown/CT 1993; Lawrence Kramer: Classical Music and Postmodern Knowledge, University of California Press: Berkeley/CA 1995; Simon Frith: Performing Rites. On the Value of Popular Music, Harvard University Press: Cambridge/MA 1996; Arthur C. Danto: After the End of Art. Contemporary Art and the Pale of History, Princeton University Press: Princeton/NJ 1997; Keith Negus: Music Genres and Corporate Cultures, Routledge: New York 1999; Jason Toynbee: Making Popular Music. Musicians, Creativity and Institutions, Arnold: London 2000; Kembrew McLeod: »Genres, Subgenres, SubSubgenres and Social Differentiation within Electronic/Dance Music Communities«, in: Journal of Popular Music Studies 13 (2001), S. 59-75; Allan F. Moore: »Categorical Conventions in Music Discourse: Style and Genre«, in: Music \& Letters 82/3 (2001), S. 432-442; Fabian Holt: »Genre Formation in Popular Music«, in: Musik \& Forskning 28 (2003), S. 77-91; Tia DeNora: »Music and Social Experience«, in: The Blackwell Companion to the Sociology of Culture, hrsg. von Mark D. Jacobs/Nancy Weiss Hanrahan, Blackwell: Oxford 2005, S. 147-159; John Frow: Genre, Routledge: New York 2006; Fabian Holt: Genre in Popular Music, University of Chicago Press: Chicago 2006; Jennifer C. Lena/Richard A. Peterson: »Classification as Culture. Types and Trajectories of Music Genres«, in: American Sociological Review 73 (2008), S. 697-718; Noël Carroll: On Criticism, Routledge: New York 2009; Jean-François Lyotard: »Music and Postmodernity « [org. frz. 1996], in: new formations 66 (2009), S. 37-45; Elijah Wald: How the Beatles Destroyed Rock 
'n' Roll. An Alternative History of American Popular Music, Oxford University Press: New York/Oxford 2009; Chris Anderton: »A Many-Headed Beast: Progressive Rock as European Meta-Genre«, in: Popular Music 29/3 (2010), S. 414-435; Chris Atton: »Popular Music Fanzines: Genre, Aesthetics, and the >Democratic Conversation«", in: Popular Music and Society 33/4 (2010), S. 517-531; Karl Hagstrom Miller: Segregating Sound: Inventing Folk and Pop Music in the Age of Jim Crow, Duke University Press: Durham/NC 2010; Peter Wicke: »Genres, Stile und musikalische Strömungen populärer Musik in Deutschland«, Bonn 2010, http://www.miz.org/static_de/themenportale/einfue hrungstexte_pdf/04_JazzRockPop/wicke_genres.pdf, S. 1-11; Chris Atton: $»$ Fan Discourse and the Construction of Noise Music as a Genre $«$, in: Journal of Popular Music Studies 23/3 (2011), S. 324-342; David Ake/Charles Hiroshi Garrett/Daniel Goldmark: »Introduction«, in: Jazz/Not Jazz, hrsg. von dies., University of California Press: Berkeley/CA 2012, S. 1-9; Chris Atton: »Genre and the Cultural Politics of Territory: The Live Experience of Free Improvisation«, in: European Journal of Cultural Studies 15/4 (2012), S. 427-441; Franco Fabbri: »How Genres Are Born, Change, Die: Conventions, Communities and Diachronic Processes«, in: Critical Musicological Reflections. Essays in Honour of Derek B. Scott, hrsg. von Stan Hawkins, Ashgate: Farnham 2012, S. 179-191; Jennifer C. Lena: Banding Together. How Communities Create Genres in Popular Music, Princeton University Press: Princeton 2012; Eric Drott: »The End(s) of Genre«, in: Journal of Music Theory 57/1 (2013), S. 1-45; Gert Keunen: Alternative Mainstream. Making Choices in Pop Music, Antennae: Amsterdam 2014; Elijah Wald: »Forbidden Sounds: Exploring the Silences of Music History« in: Geschichte wird gemacht, hrsg. von Dietrich Helms/Thomas Phleps, transcript: Bielefeld 2014, S. 25-39; David Brackett: »Popular Music Genres: Aesthetics, Commerce and Identity«, in: The SAGE Handbook of Popular Music, hrsg. von Andy Bennett/Steve Waksman, Thousand Oaks/ CA: SAGE 2015, S. 189-206; Gabriele Marino: ") What Kind of Genre Do You Think We Are?^ Genre Theories, Genre Names and Classes within Music Intermedial Ecology«, in: Music Analysis Experience. New Perspectives in Musical Semiotics, hrsg. von Constantino Maeder/Mark Reybrouck, Leuven University Press: Leuven 2015, S. 239-254; Klaus Nathaus: »From Versatility to Art and Authenticity. The Making of Popular Music's Genre Matrix, 18901930«, in: MusikTheorie 30/1 (2015), S. 23-41; David Brackett: Categorizing Sound. Genre and Twentieth-Century Popular Music, University of California Press: Berkeley/CA 2016; Frédéric Döhl: Mashup in der Musik. Fremdrefe- 
renzielles Komponieren, Sound Sampling und Urheberrecht, transcript: Bielefeld 2016; Robin James: »Is the Post- in Post-Identity the Post- in PostGenre? «, in: Popular Music 36/1 (2017), S. 21-32.

11 Genrebestimmung nach Frédéric Döhl: Mashup in der Musik. Fremdreferenzielles Komponieren, Sound Sampling und Urheberrecht, transcript: Bielefeld 2016, S. 131f. unter Bezugnahme insbesondere auf Stephen Neale: Genre, British Film Institute: London 1980, S. 19-22; Franco Fabbri, »A Theory of Musical Genres: Two Applications«, in: Popular Music Perspectives, hrsg. von David Horn/Philipp Tagg, IASPM: Gothenburg/Exeter 1982, S. 52-81, hier S. 52, 55; Sarah Thornton: Club Cultures. Music Media and Subcultural Capital, Polity Press: Cambridge 1995, S. 14; Keith Negus: Music Genres and Corporate Cultures, Routledge: New York 1999, S. 25; Christine Glendhill: »Rethinking Genre«, in: Reinventing Film Studies, hrsg. von Christine Gledhill/Linda Williams, Arnold: London 2000, S. 221-243, hier S. 223; Jim Samson: »Genre«, in: Grove Music Online, 2001 (Abruf am 7. August 2014); Kembrew McLeod: »Genres, Subgenres, Sub-Subgenres and Social Differentiation within Electronic/Dance Music Communities«, in: Journal of Popular Music Studies 13 (2001), S. 59-75, hier S. 60; Stuart Borthwick/Ron Moy: Popular Music Genres: An Introduction, Edinburgh University Press: Edinburgh 2004, S. 3; Roy Shuker: »genre; meta genres; subgenres«, in: ders., Popular Music: The Key Concepts, 2. Auflg., Routledge: New York 2005, S. 145-149, hier S. 147f.; John Frow: Genre, Routledge: New York 2006, S. 10; Fabian Holt: Genre in Popular Music, University of Chicago Press: Chicago 2007, S. 2; Jennifer C. Lena/Richard A. Peterson: »Classification as Culture. Types and Trajectories of Music Genres«, in: American Sociological Review 73 (2008), S. 697-718, hier S. 698; Jennifer C. Lena: Banding Together. How Communities Create Genres in Popular Music, Princeton University Press: Princeton/NJ 2012, S. 6; David Hesmondhalgh: The Cultural Industries, 3. Auflg., SAGE: Los Angeles/CA 2013, S. 32.

12 Simon Frith: Performing Rites. On the Value of Popular Music, Harvard University Press: Cambridge/MA 1996, S. 87, 94.

13 Vgl. Pierre Bourdieu: „Ökonomisches Kapital, kulturelles Kapital, soziales Kapital«, in: Soziale Ungleichheiten, hrsg. von Reinhard Kreckel, Schwartz: Göttingen 1983, S. 183-198, insb. S. 185.

14 Vgl. Wilhelm Altmann: Handbuch für Klavierquintettspieler. Wegweiser durch die Klavierquintette, Verlag für musikalische Kultur und Wissenschaft: Wolfenbüttel 1936, S. 24; Basil Smallman: The Piano Quartet and Quintet. 
Style, Structure, and Scoring, Clarendon Press: Oxford 1994, S. 53; Gottfried Heinz: Die Geschichte des Klavierquintetts von den Anfängen bis Robert Schumann, Männeles: Neckargemünd 2001, S. 1, 216; Joanne Richardson: The Piano Quintet. Influence of Medium on Genre [Diss.], City University London: London 2014, http://openaccess.city.ac.uk/3163/, S. 51.

15 Vgl. Basil Smallman: The Piano Quartet and Quintet. Style, Structure, and Scoring, Clarendon Press: Oxford 1994, S. 51; Gottfried Heinz: Die Geschichte des Klavierquintetts von den Anfängen bis Robert Schumann, Männeles: Neckargemünd 2001, S. 216.

16 Vgl. Basil Smallman: The Piano Quartet and Quintet. Style, Structure, and Scoring, Clarendon Press: Oxford 1994, S. 51; Klaus Aringer: »Deutsche Romantik«, in: Die Sonate: Formen instrumentaler Ensemblemusik (= Handbuch der musikalischen Gattung, Bd. 5), hrsg. von Claus Brockmaier/Siegfried Mauser, Laaber: Laaber 2005, S. 263-284, hier S. 270.

17 Vgl. Basil Smallman: The Piano Quartet and Quintet. Style, Structure, and Scoring, Clarendon Press: Oxford 1994, S. 51; Gottfried Heinz: Die Geschichte des Klavierquintetts von den Anfängen bis Robert Schumann, Männeles: Neckargemünd 2001, S. 1.

18 Vgl. Colin Lawson: »The String Quartet as a Foundation for Larger Ensembles«, in: The Cambridge Companion to the String Quartet, hrsg. von Robin Stowell, Cambridge University Press: Cambridge 2003, S. 310-327, hier, S. 323.

19 Vgl. zu dieser Werkgruppe innerhalb der Gattung mit Klavierquintetten u.a. von Max Bruch, Friedrich Gernsheim, Hermann Goetz, Karl Goldmark, Heinrich von Herzogenberg, Friedrich Kiel, August Klughardt, Joachim Raff, Carl Reinecke, Josef Rheinberger oder Ferdinand Thieriot weiter Michael Aschauer: Einheit durch Vielfalt? Das Klavierkammermusikwerk ausgewählter »Konservativer um Johannes Brahms: Klaviertrios, Klavierquartette und Klavierquintette von Robert Fuchs, Hermann Goetz, Karl Goldmark, Heinrich von Herzogenberg, Josef Gabriel Rheinberger, Richard Strauss und Robert Volkmann, Peter Lang: Frankfurt am Main 2006.

20 Joël-Marie Fauquet: »Chamber Music in France. From Cherubini to Debussy«, in: Nineteenth-Century Chamber Music, hrsg. von Stephen E. Hefling, Routledge: New York 1998, S. 287-314, hier S. 303f.

21 Klaus Aringer: »Deutsche Romantik«, in: Die Sonate: Formen instrumentaler Ensemblemusik (= Handbuch der musikalischen Gattung, Bd. 5), hrsg. von 
Claus Brockmaier/Siegfried Mauser, Laaber: Laaber 2005, S. 263-284, hier S. 270 .

22 Gut eine Generation, nachdem Ignaz Schuppanzigh, ein wichtiger künstlerischer Partner Beethovens, 1808 in Wien das sogenannte Rasumowsky-Quartett formiert hatte, das als erstes seiner Art gilt. Vgl. Hans Heinrich Eggebrecht (Hrsg.), Riemann Musik Lexikon, Sachteil, Mainz 1967, S. 912. Freilich wechselte seine Besetzung wiederholt, vgl. Jan Caeyers: Beethoven: Der einsame Revolutionär. Eine Biographie, C.H. Beck: München 2012, S. 392f. Diese Rahmenbedingung machen das Klavierquintett als Gattung dabei besonders attraktiv: Fast alle berühmten Pianisten des 20. Jahrhunderts von Swjatoslaw Richter bis Glenn Gould und Streichquartette vom Julliard Quartet bis zum Emerson String Quartet haben Klavierquintette aufgeführt und aufgenommen.

23 Vgl. einführend Wilhelm Altmann: Handbuch für Klavierquintettspieler. Wegweiser durch die Klavierquintette, Verlag für musikalische Kultur und Wissenschaft: Wolfenbüttel 1936; Basil Smallman: The Piano Quartet and Quintet. Style, Structure, and Scoring, Clarendon Press: Oxford 1994; Gottfried Heinz: Die Geschichte des Klavierquintetts von den Anfängen bis Robert Schumann, Männeles: Neckargemünd 2001; Joanne Richardson: The Piano Quintet. Influence of Medium on Genre [Diss.], City University London: London 2014, http://openaccess.city.ac.uk/3163/ sowie die im letzten Kapitel genannten Aufsätze zu Einzelwerken.

24 Damit sind die ihrem Selbstverständnis wie ihrer öffentlichen Wirkung nach maßgeblich für Kanonisierung verantwortlichen Instanzen benannt. Eine Differenzierung, die Autoren wie Joseph Kerman oder Carl Dahlhaus mit Vehemenz einforderten. Vgl. Joseph Kerman: »A Few Canonic Variations«, in: Critical Inquiry 10 (1983/84), S. 107-125, insb. S. 111; Carl Dahlhaus: »Historik - Grundlagen der Musikgeschichte - Ästhetik«, in: Gesammelte Schriften, Bd. 1, hrsg. von Hermann Danuser, Laaber: Laaber 2000, S. 11-155, insb. S. 97.

25 Udo Göttlich: »Massenmedium«, in: Metzler Lexikon. Medientheorie Medienwissenschaft, hrsg. von Helmut Schanze, J.B. Metzler: Stuttgart 2002, S. $193 f$.

26 Noël Carroll: Art in Three Dimensions, Oxford University Press: Oxford/New York 2010, S. 47.

27 Vgl. zur Klassikbetrieb Nina Polaschegg: Populäre Klassik-Klassik populär: Hörerstrukturen und Verbreitungsmedien im Wandel, Böhlau: Köln 2005; Dawn Bennett: Understanding the Classical Music Profession. The Past, the Present and Strategies for the Future, Ashgate: Aldershot 2008; Michael 
Custodis: Klassische Musik heute. Eine Spurensuche in der Rockmusik, transcript: Bielefeld 2009; Jörn Peter Hiekel (Hrsg.): Populär vs. elitär. Wertvorstellungen und Popularisierungen der Musik heute, Schott: Mainz 2013; Martin Lücke: »Der deutsche Klassikmarkt: Eine wirtschaftliche Betrachtung«, in: Musikwirtschaft 2.0. Bestandsaufnahme und Perspektiven, hrsg. von Steffen Höhne/Matthias Maier/Wolf-Georg Zaddach, Leipziger Universitätsverlag: Leipzig 2014, S. 71-92; Marius Carboni: »The Classical Music Business«, in: The Music Industry Handbook, 2. Auflg. hrsg. von Paul Rutter, Routledge: New York 2016, S. 195-223; Chris Dromey/Julia Haferkorn (Hrsg.): The Classical Music Industry, Routledge: New York 2018; Gregor Herzfeld (Hrsg.): Popularisierung »klassischer« Musik = Musiktheorie 33/4 (2018), S. 282-368; Christian Kellersmann: »Die ıneue Klassik«", in: Das Konzert II. Beiträge zum Forschungsfeld der Concert Studies, hrsg. von Martin Tröndle, transcript: Bielefeld 2018, S. 379-387 (erweiterte Fassung von Christian Kellersmann: „Wege der Erneuerung«, in: Das Konzert I. Neue Aufführungskonzepte für eine klassische Form, 2. Auflg., hrsg. von Martin Tröndle, transcript: Bielefeld 2011, S. 221-226); Barbara Balba Weber: Entfesselte Klassik. Grenzen öffnen mit künstlerischer Musikvermittlung, Stämpfli und Cie.: Bern 2018.

28 Vgl. Marius Carboni: »The Classical Music Business«, in: The Music Industry Handbook, 2. Auflg. hrsg. von Paul Rutter, Routledge: New York 2016, S. 195-223, hier S. 202f.; William Robin: »How a Somber Symphony Sold More Than a Million Records«, in: The New York Times (9. Juni 2017), https:// www.nytimes.com/2017/06/09/arts/music/how-a-somber-symphony-sold-mor e-than-a-million-records.html.

29 Vgl. Marius Carboni: »The Classical Music Business«, in: The Music Industry Handbook, 2. Auflg. hrsg. von Paul Rutter, Routledge: New York 2016, S. 195-223, hier S. 195-203; Eve Klein: »When Divas and Rock Stars Collide: Interpreting Freddie Mercury and Montserrat Caballé's Barcelona«, in: Popular Music, Stars and Stardom, hrsg. von Stephen Loy/Julie Rickwood/Samantha Bennett, Australian National University Press: Canberra 2018, S. 115-135, hier S. 116-119.

30 Vgl. Theodor W. Adorno: »Kulturindustrie. Aufklärung als Massenbetrug«, in: Theodor W. Adorno, Gesammelte Schriften Bd. 3, hrsg. von Rolf Tiedemann, Suhrkamp: Frankfurt am Main 21984, S. 19-60 (mit Max Horkheimer als Dialektik der Aufklärung); Pierre Bourdieu: Die feinen Unterschiede. Kritik der gesellschaftlichen Urteilskraft, Suhrkamp: Frankfurt am Main 2003; Gerhard 
Schulze: Die Erlebnisgesellschaft. Kultursoziologie der Gegenwart, 2. Auflg., Campus: Frankfurt 2005; Hartmut Böhme: Fetischismus und Kultur. Eine andere Theorie der Moderne, Rowohlt: Reinbek bei Hamburg 2006.

31 Vgl. Colin Lawson: »The String Quartet as a Foundation for Larger Ensembles«, in: The Cambridge Companion to the String Quartet, hrsg. von Robin Stowell, Cambridge University Press: Cambridge 2003, S. 310-327, hier, S. 324-327.

32 Vgl. 1984, Philips 411 107-1 (Elena Bashkirova, Gideon Kremer, Kathrin Rabus, Gerard Caussé, Ko Iwaski); 1991, Virgin Classics 0777-7590402-4 (Ludmilla Berlinsky, Borodin String Quartet); 1991, Etcetera KTC 1124 (Fred Oldenburg, Mondriaan String Quartet); 1992, BIS CD-547 (Roland Pöntinen, The Tale Quartet); 1993, Sony Classical SK 53357 (Irina Schnittke, Mark Lubotsky, Tamaz Batiashvili, Grazyna Filipajtis-Lubotsky, Karl-Bernhard von Stumpff); 1994, Russian Disc RD CD 10031 (Constantine Orbelian, Moscow String Quartet); 1995, Globe 5069 (The Harmonies of the World); 1996, Amati AMI9304 (Maida Magomedbekowa, Lysenko-Quartett); 1996, Erasmus Muziekproducties WVH170 (Doelen Ensemble); 1996, Hyperion CDA66885 (Capricorn); 1998, BMG 74321562642 (Yuri Smirnov, Gideon Kremer, Tatiana Grindenko, Yuri Bashmet, Karina Georgian - Aufnahme von 1977); 1998, Arabesque Recordings Z6707 (Gary Graffman, The Lark Quartet); 1999, Naxos 8.554728 (AFCM Ensemble); 2000, Marquis Classics (Lev Natochenny, Penderecki String Quartet); 2000, Naxos 8.554830 (Boris Berman, Vermeer Quartet); 2001, ASV Quicksilva CD QS 6251 (Barbican Piano Trio, Jan Peter Schmolck, James Boyd) = 2004, Black Box BBM1093; 2002, Northern Flowers NF/PMA 9908 (Yuri Serov, Lidia Kovalenko, Alexey Baev, Alexey Popov, Kirill Timofeev); 2003, ECM 4618152 (Alexei Lubimov, Keller Quartett); 2011, Crystal Classics N 67083 (Ewa Kupiec, Petersen Quartett) = 2012, Delta Classics DLT 90083; 2011, EMI Classics 5099907083624 (Lilya Zilberstein, Dora Schwarzberg, Lucia Hall, Lida Chen, Jorge Bosso); 2013, ATMA Classique ACD 22669 (Louise Bessette, Quatuor Molinari); 2014, Melodiya MEL CO 0172 (Eliso Virsaladze, Borodin Quartet - Aufnahme von 1986); 2016, Odradek ODRCD341 (Erato Alakiozidou, Lutosławski Quartet).

33 Vgl. auch 1996, ASV CD DCA 678 (Allan Schiller, Coull String Quartet); 2009, Hyperion CDA67726 (Piers Lane, Goldner String Quartet); 2009, Somm Recordings SOMMCD 087 (Michael Dussek, Bridge Quartet); 2010, Dutton Vocalion CDLX 7254 (London Bridge Ensemble); 2010, Naxos 8.572474 
(Ashley Wass, The Tippett Quartet); 2015, Naxos 8.571355 (Raphael Terroni, Bingham String Quartet - Reissue, Aufnahme von 1989).

34 Vgl. Ulrike Altig/Michel Clement/Dominik Papies, »Marktübersicht und Marktentwicklung der Musikindustrie«, in: Ökonomie der Musikindustrie, hrsg. von Michel Clement/Oliver Schusser/Dominik Papies, 2. Auflg., Gabler: Wiesbaden 2008, S. 17-26, hier S. 24; Paul Rutter: The Music Industry Handbook, New York 2011, S. 113, 118; Geoffrey P. Hull/Thomas Hutchinson/ Richard Strasser, The Music Business and Recording Industry, 3. Auflg., Routledge: New York 2011, S. 174; Tim J. Anderson: Popular Music in a Digital Music Economy. Problems and Practices for an Emerging Service Industry, Routledge: New York 2014, S. 7.

35 Vgl. https://portal.dnb.de/opac.htm? method=moveDown\&currentResultId=\%2 2 klavierquintett $\% 22 \% 26 \mathrm{dnb}$.dma\&categoryId=music. Suche im Katalog des Deutschen Musikarchivs nach »Klavierquintett«.

36 Die Angaben wurden zusammengetragen aus den Angaben in den Katalogen auf den Websites der drei Labels plus den Angeboten auf Amazon, JPC, discogs.com, arkivmusic.com, musicline.de (Phononet) und im Bielefelder Katalog.

37 Vgl. https://soundcloud.com/search/sounds?q=\%22piano\%20quintet\%22; http s://soundcloud.com/search/albums?q=\%22piano\%20quintet $\% 22 ;$ https://sound cloud.com/search/sets?q=\%22piano\%20quintet\%22. Suche nach »Piano Quintet« (oder »Klavierquintett $«)$ in Tracks, Alben und Playlists.

38 Vgl. http://www.ard.de/home/radio/Was_lief_wann_wo_/96872/index.html.

39 Vgl. Gerald Spindler: »Text und Data Mining - urheber- und datenschutzrechtliche Fragen«, in: GRUR (2016), S. 1112-1120; Benjamin Raue: »Text und Data Mining«, in: $C R$ (2017), S. 656-662; Benjamin Raue: »Urheberrecht der digitalen Wissen(schaft)sgesellschaft«, in: GRUR (2017), S. 11-19; Fabian Rack: »Music Data Mining und das Urheberrecht«, in: Jahrbuch für Musikwirtschafts- und Musikkulturforschung 1 (2018), S. 23-51.

40 Vgl. Frédéric Döhl: »Potential und Risiken des Archival Turns in den Digital Humanities für die Musikwissenschaft«, in: Archiv für Musikwissenschaft 75/4 (2018), S. 301-320.

41 Vgl. https://www.youtube.com/results?search_query=\%22Piano+Quintet $\% 22$ $\& \mathrm{sp}=\mathrm{CAM} \% 253 \mathrm{D}$.

42 Vgl. https://www.youtube.com/watch?v=q-Of5GTSBPk. Aus der Neoklassik kommen im Übrigen auch weitere Beiträge von außerhalb der Liste, aber 
innerhalb des Genres, die Popmusikzahlen erreichen, so Chilly Gonzales' $»$ Advantage Points« $(\sim 577.000)$, wie noch Thema sein wird.

43 Ich danke herzlich Bernhard Neuhoff und Martina Bulla für die freundliche Unterstützung durch die Erstellung einer entsprechenden Repertoirestatistik aus dem internen Archivsystem.

44 Vgl. stellvertretend zum Bereich Konzerte und Konzertprogramm Barbara Wiermann: »musiconn.performance - Musikalische Ereignisdaten im Fachinformationsdienst Musikwissenschaft«, in: Kooperative Informationsstrukturen als Chance und Herausforderung, hrsg. von Achim Bonte/Juliane Rehnolt, De Gruyter: Berlin 2018, S. 398-415.

45 Vgl. http://www.miz.org/statistiken/konzerte-musiktheater-s1511.

46 E-Mails von Andrea Hammes (SLUB Dresden, 7.3.2019) und Jens Kindermann (GEMA, 18.3.2019) an den Autor.

47 Vgl. stellvertretend zum Bereich Konzerte und Konzertprogramm Barbara Wiermann: »musiconn.performance - Musikalische Ereignisdaten im Fachinformationsdienst Musikwissenschaft«, in: Kooperative Informationsstrukturen als Chance und Herausforderung, hrsg. von Achim Bonte/Juliane Rehnolt, De Gruyter: Berlin 2018, S. 398-415, hier S. 404-409.

48 Vgl. https://www.musikverein.at/konzertarchiv; https://www.carnegiehall.org/ About/History/Performance-History-Search?q=\&dex=prod_PHS.

$49 \mathrm{Vgl}$. http://www.miz.org/themenportale/konzerte-musiktheater/konzerthaeuser -s119; http://www.miz.org/themenportale/konzerte-musiktheater/musikfestspie le-festwochen-festivals-s626http://www.miz.org/themenportale/konzerte-musi ktheater/konzertdirektionen-kuenstleragenturen-s558.

50 Vgl. https://issuu.com/search?q=klavierquintett.

51 Vgl. http://www.musikadler.de/suche.html.

52 Vgl. http://www.spectrumconcerts.com/home/\%C3\%BCber-spectrum/1988-20 19-repertoire/.

53 Vgl. https://issuu.com/concerti.

54 Vgl. Hans Heinrich Eggebrecht: Musik im Abendland, Piper: München 1996; Alex Ross: The Rest is Noise: Das 20. Jahrhundert hören, Piper: München 2009; Werner Keil: Musikgeschichte im Überblick, 3. Auflg., UTB: Paderborn 2018.

55 Vgl. Carl Dahlhaus (Hrsg.): Neue Handbuch der Musikwissenschaft. Bd. 13: Register, Laaber: Laaber 1995.

56 Vgl. Richard Taruskin: Music in the Nineteenth Century, Oxford University Press: New York/Oxford 2010, S. 139, 731, 754, 776. 
Vgl. J. Peter Burkholder/Donald Jay Grout/Claude V. Palisca: A History of Western Music, 9. Auflg., Norton: New York 2014, S. 598, 728, 754.

58 Vgl. Paul Griffiths: A Concise History of Western Music, Cambridge University Press: Cambridge 2009, S. 186, 208.

59 Vgl. Walter Frisch: Music in the Nineteenth Century. Western Music in Context, Norton: New York 2013, S. 46, 179.

60 Vgl. Jim Samson (Hrsg.): The Cambridge History of Nineteenth-Century Music, Cambridge University Press: Cambridge 2002, S. 190, 203, 504.

61 Vgl. Matthew Rye: 1001 Klassik-Alben, die sie hören sollten, bevor das Leben vorbei ist, Ed. Olms: Zürich 2008; Arnold Werner-Jensen: Das Reclam Buch der Musik, 3. Auflg., Reclam: Stuttgart 2012; Kurt Pahlen: Die große Geschichte der Musik, Cormoran: München 1998.

62 Vgl. Basil Smallman: The Piano Quartet and Quintet. Style, Structure, and Scoring, Clarendon Press: Oxford 1994; Gottfried Heinz: Die Geschichte des Klavierquintetts von den Anfängen bis Robert Schumann, Männeles: Neckargemünd 2001; Michael Aschauer, Einheit durch Vielfalt? Das Klavierkammermusikwerk ausgewählter »Konservativer « um Johannes Brahms: Klaviertrios, Klavierquartette und Klavierquintette von Robert Fuchs, Hermann Goetz, Karl Goldmark, Heinrich von Herzogenberg, Josef Gabriel Rheinberger, Richard Strauss und Robert Volkmann, Peter Lang: Frankfurt am Main 2006.

63 Vgl. für einen Überblick Christine Lemke-Matwey: »Ektasen mit viel Rosenwasser. Richard Wagner wird 200 - und die Wagner-Literatur dreht sich emsig im Kreis«, in: DIE ZEIT 41 (13. November 2012), http://www.zeit.de/2012/ 41/Richard-Wagner-200-Geburtstag-Literatur; Wolfram Goertz: »Wie klingen Gier, Wonne, Tod? Zum 200. Geburtstag von Richard Wagner gibt es Berge von Büchern. Über die Musik findet sich darin - fast nichts!«, in: DIE ZEIT 31 (7. August 2013), http://www.zeit.de/2013/31/literatur-sachbuch-richard-wagn er-200-jahre.

64 Vgl. zuletzt etwa Alexander Rehding: Beethoven's Symphony No. 9, Oxford University Press: New York/Oxford 2017; Christopher Alan Reynolds: Wagner, Schumann, and the Lessons of Beethoven's Ninth, University of California Press: Berkeley/CA 2015; Harvey Sachs: The Ninth. Beethoven and the World in 1824, Faber and Faber: London 2010; Christina M. Stahl: Was die Mode streng geteilt?! Beethovens Neunte während der deutschen Teilung, Schott: Mainz 2009; Michael Wasserman: Le sacre de l'hiver. La Neuvième Symphonie de Beethoven, un mythe de la modernité japonaise, Les Indes Savantes: Pa- 
ris 2006; Dieter Hildebrandt: Die Neunte. Schiller, Beethoven und die Geschichte eines musikalischen Welterfolgs, Hanser: München 2005; Mario Bois: Beethoven et l'hymne de l'Europe. Genèse et destin de l'Hymne à la joie, Séguier: Biarritz 2005; Gianfranco Belardini: La nona sinfonia de Beethoven. Una storia universale, Il Candelaio: Florenz 2004; David Benjamin Levy: Beethoven: The Ninth Symphony, Yale University Press: New Haven 22003; Esteban Buch: Beethovens Neunte. Eine Biographie, Propyläen: Berlin 2000; Karl-Heinz Köhler: ...Tochter aus Elysium. Werden und Uraufführung der Neunten Sinfonie Ludwig van Beethovens und die abenteuerlichen Wege des zerteilten Autographs, Peter Lang: Frankfurt am Main 2000; Nicholas Cook: Beethoven: Symphony No. 9, Cambridge University Press: Cambridge 1993; Andreas Eichhorn: Beethovens Neunte Symphonie. Die Geschichte ihrer Aufführung und Rezeption, Bärenreiter: Kassel 1993; Johannes Bauer: Rhetorik der Überschreitung. Annotationen zu Beethovens Neunter Symphonie, Centaurus: Pfaffenweiler 1992; Dieter Rexroth: Ludwig van Beethoven, Symphonie Nr. 9 d-Moll op. 125, 2. Auflg., Schott: Mainz 1988.

65 Vgl. Basil Smallman/Christina Nobach/Michael Töpel: »Klavierkammermusik«, in: Die Musik in Geschichte und Gegenwart, 2. Auflg., Sachteil Bd. 5, hrsg. von Ludwig Finscher, Kassel/Stuttgart 1996, S. 326-346; Ludwig Finscher, »Gattungen und Besetzungen der Kammermusik«, in: Reclams Kammermusikführer, 13. Auflg., hrsg. von Arnold Werner-Jessen, Reclam: Stuttgart 2005, S. 11-132, hier S. 88-92 (vgl. zum Streichquartett S. 101-116).

66 Vgl. David Fenton: »Piano Quintet«, in: New Grove Dictionary of Music and Musicians. Second Edition, hrsg. von Stanley Sadie, MacMillan: London 2001. Abruf über Grove Music Online.

67 Vgl. auch John H. Baron: Chamber Music. A Research and Information Guide, 3. Auflg., Routledge: New York 2010, S. 29, 121-124.

68 Vgl. Handbuch der musikalischen Gattungen (div. wechselnde Hrsg. je Band) Laaber: Laaber 2000-2010; Handbuch der Musik im 20. Jahrhundert (div. wechselnde Hrsg. je Band), Laaber 1999-2011.

69 Vgl. Dorothea Redepenning: Geschichte der russischen und sowjetischen Musik, 3. Bd., Laaber: Laaber 1994/2008.

70 Vgl. Ingeborg Allihn (Hrsg.): Kammermusikführer, dtv/Bärenreiter: Kassel/ München 2000; Meyers Lexikonverlag (Hrsg.): Harenberg Kulturführer Kammermusik, 3. Auflg., Meyers Lexikonverlag: Mannheim ${ }^{3} 2008$; Melvin Berger: Guide to Chamber Music, Dover: Mineola/NY 2001; James M. Keller: Chamber Music: A Listener's Guide, Oxford University Press: New York/ 
Oxford 2011; Lucy Miller Murray: Chamber Music: An Extensive Guide for Listeners, Scarecrow: Lanham/MD 2015.

71 Vgl. Colin Lawson: »The String Quartet as a Foundation for Larger Ensembles«, in: The Cambridge Companion to the String Quartet, hrsg. von Robin Stowell, Cambridge University Press: Cambridge 2003, S. 310-327, hier, S. 324-327.

72 Vgl. Heinz Josef Herbort: »Tod und Verklärung«, in: DIE ZEIT 36 (2.9.1994), http://www.zeit.de/1994/36/tod-und-verklaerung/seite-4; Heinz Josef Herbort: »Kein Randbezirk von Byzanz mehr«, in: DIE ZEIT 36 (1.9.1995), http:// www.zeit.de/1995/36/Kein_Randbezirk_von_Byzanz_mehr/seite-4; Michael Naura: »Zeit zum Hören«, in: DIE ZEIT 52 (22.12.1995), http://www.zeit.de/ 1995/52/Zeit_zum_Hoeren; Andreas Nentwich: »Im Kerker des Zweifels«, in: DIE ZEIT 43 (19.10.2000), http://www.zeit.de/2000/43/Im_Kerker_des_Zwei fels; Oswald Beaujean: »Inwendig glühend«, in: DIE ZEIT 26 (20.6.2002), http://www.zeit.de/2002/26/Inwendig_gluehend; Oswald Beaujean: »Schostakowitschs Schatten«, in: DIE ZEIT 12 (13.3.2003), http://www.zeit.de/feuille ton/kulturbrief/kulturbrief_140303; Volker Hagedorn: »Raff in Form«, in: DIE ZEIT 36 (28.8.2003), http://www.zeit.de/2003/36/Raff_in_Form; Frank Hilberg, »Musik. In der Meisterschmiede«, in: DIE ZEIT 4 (21.1.2004), http:// www.zeit.de/feuilleton/kulturbrief/2004/04/tontraeger1; Anonymous: »Kon zerte«, in: DIE ZEIT 17 (21.4.2004), http://www.zeit.de/feuilleton/kulturbrief/ 2004/17/konzerte; Volker Hagedorn: »Allein mit seinem Willen«, in: DIE ZEIT 49 (25.11.2004), http://www.zeit.de/2004/49/Allein_mit_seinem_Wil len/seite-2; Christine Lemke-Matwey: »Klassik. Scheppert wie im Westen«, in: DIE ZEIT 31 (27.7.2006), http://www.zeit.de/2006/31/D-Aufmacher; Volker Hagedorn: »Die Klassik-Platte. Forelle Blau«, in: DIE ZEIT 12 (14.4. 2009), http://www.zeit.de/2013/07/CD-DVD-Jazz-Klassik-Pop-Film; Wolfram Goertz: »Pianist Éric Le Sage«, in: DIE ZEIT 35 (31.8.2010), http://www. zeit.de/2010/35/Pianist-Le-Sage; Volker Hagedorn: »Komponist Gabriel Fauré«, in: DIE ZEIT 41 (4.11.2011), http://www.zeit.de/2011/41/M-Faure; Volker Hagedorn: »August, der Starke«, in: DIE ZEIT 7 (7.2.2013), http://www. zeit.de/2013/07/CD-DVD-Jazz-Klassik-Pop-Film.

73 Carl Dahlhaus: »Was ist Musikgeschichte?«, in: Europäische Musikgeschichte, Bd. 1, hrsg. von Sabine Ehrmann-Herfort/Ludwig Finscher/Giselher Schubert, Bärenreiter/Metzler: Kassel/Stuttgart 2002, S. 59-79, hier S. 65.

74 Friedrich Geiger/Tobias Janz: »Ökonomie und Kanon«, in: Der Kanon der Musik. Theorie und Geschichte. Ein Handbuch, hrsg. von Klaus Pietschmann/ 
Melanie Wald-Fuhrmann, edition text + kritik: München 2013, S. 858-888, hier S. $862 f$.

75 Friedrich Geiger/Tobias Janz: »Ökonomie und Kanon«, in: Der Kanon der Musik. Theorie und Geschichte. Ein Handbuch, hrsg. von Klaus Pietschmann/ Melanie Wald-Fuhrmann, edition text + kritik: München 2013, S. 858-888, hier S. 862.

76 Vgl. Carl Dahlhaus: „Was ist Musikgeschichte?«, in: Europäische Musikgeschichte, Bd. 1, hrsg. von Sabine Ehrmann-Herfort/Ludwig Finscher/Giselher Schubert Bärenreiter/Metzler: Kassel/Stuttgart 2002, S. 59-79, hier S. 60. Vgl. einführend zum Thema einschließlich einer Übersicht über die Standardliteratur zum Thema Melanie Wald-Fuhrmann: »Musikwissenschaft«, in: Handbuch Kanon und Wertung, hrsg. von Gabriele Rippl/Simone Winko, J.B. Metzler: Stuttgart 2013, S. 371-379.

77 Vgl. Narasimhan Anand/Richard A. Peterson: »The Production of Culture Perspective«, in: Annual Review of Sociology 30 (2004), S. 311-334.

78 Melanie Wald-Fuhrmann: »Musikwissenschaft«, in: Handbuch Kanon und Wertung, hrsg. von Gabriele Rippl/Simone Winko, J.B. Metzler: Stuttgart 2013, S. 371-379, hier S. 378.

79 Vgl. einführend zu den Methoden der Digital Humanities Ruth Reiche/Rainer Becker/Michael Bender/Mathew Munson/Stefan Schmunk/Christof Schöch: Verfahren der Digital Humanities in den Geistes- und Kulturwissenschaften, GOEDOC/DARIAH_DE: Göttingen 2014, http://webdoc.sub.gwdg.de/pub/ mon/dariah-de/dwp-2014-4.pdf; Tanya E. Clement: »Where Is Methodology in Digital Humanities?«, in: Debates in the Digital Humanities, hrsg. von Matthew Gold/Lauren F. Klein, 2016, http://dhdebates.gc.cuny.edu/debates/text/ 65; Susanne Kurz: Digital Humanities. Grundlagen und Technologien für die Praxis, 2. Auflg., Springer: Wiesbaden 2016; Lorna Hughes/Panos Constantopoulos/Costis Dallas: »Digital Methods in the Humanities: Understanding and Describing their Use across the Disciplines«, in: A New Companion to Digital Humanities, hrsg. von Susan Schreibman/Ray Siemens/John Unsworth, Wiley Blackwell: Malden/MA 2016, S. 150-170; Angela Dressen: »Grenzen und Möglichkeiten der digitalen Kunstgeschichte und der Digital Humanities eine kritische Betrachtung der Methoden«, in: Critical Approaches to Digital Art History = kunsttexte.de Nr. 4, 2017, https://edoc.huberlin.de/bitstream/han dle/18452/19402/Angela\%20Dressen\%20-\%20final.pdf; Fotis Jannidis/Hubertus Kohle/Malte Rehbein: Digital Humanities. Eine Einführung, J.B. Metzler: 
Stuttgart 2017; Lewis Levenberg/Tai Neilson/David Rheams (Hrsg.): Research Methods for the Digital Humanities, Palgrave Macmillan: Cham 2018.

80 Vgl. http://dig-that-lick.eecs.qmul.ac.uk/.

81 Vgl. Andrew Goldstone/Ted Underwood: »The Quiet Transformations of Literary Studies: What Thirteen Thousand Scholars Could Tell Us«, in: New Literary History 45/3 (2014), S. 359-384, https://www.ideals.illinois.edu/bit stream/handle/2142/49323/QuietTransformations.pdf?sequence=2\&isAllowed $=\mathrm{y}$.

82 Vgl. Ossi Naukkarinen/Darius Pacauskas: »Aesthetics in Digital Worlds«, in: Contemporary Aesthetics 16 (2018), https://contempaesthetics.org/newvolume /pages/article.php?articleID=837. Vgl. für eine Beispieluntersuchung Ralf von Appen: Der Wert der Musik. Zur Ästhetik des Populären, transcript: Bielefeld 2007.

83 Man kann sogar darauf hoffen, dass dereinst ein technisches und methodisches Level erreicht wird, auf dem mit Computerunterstützung großangelegte Werkund Interpretationsvergleiche möglich werden, um z.B. quantitativ zu überprüfen, ob hinter Kernrepertoirebildung und Qualitätsunterstellung benennbare Differenzen in kompositorischer Machart oder ästhetischen Reaktionen zwischen den Genrebeiträgen stehen. Vgl. Johanna Barzen/Uwe Breitenbücher/ Linus Eusterbrock/Michael Falkenthal/Frank Hentschel/Frank Leymann: »The Vision for MUSE4Music«, in: Computer Science - R\&D 32/3-4 (2017), S. 323-328.

84 Vgl. Bruno Latour: »Why Has Critique Run Out of Steam? From Matters of Fact to Matters of Concern«, in: Critical Inquiry 30/2 (2004), S. 225-248, hier S. 237-242.

85 https://www.fu-berlin.de/sites/dhc/programme/termine/dh-gespraech-sose19-3. html.

86 Anselm Gerhard: ")Kanon< in der Musikgeschichtsschreibung. Nationalistische Gewohnheiten nach dem Ende der nationalistischen Epoche«, in: Der Kanon der Musik. Theorie und Geschichte. Ein Handbuch, hrsg. von Klaus Pietschmann/Melanie Wald-Fuhrmann, München 2013, S. 54-71, hier S. 68.

87 Joseph Kerman: Contemplating Music: Challenges to Musicology, Harvard University Press: Cambridge/MA, S. 12. Vgl. im Übrigen die Einleitung ebd., S. 11-30, und Kap. 4, S. 113-154.

88 Anselm Gerhard: ")Kanon< in der Musikgeschichtsschreibung. Nationalistische Gewohnheiten nach dem Ende der nationalistischen Epoche«, in: Der Kanon der Musik. Theorie und Geschichte. Ein Handbuch, hrsg. von Klaus 
Pietschmann/Melanie Wald-Fuhrmann, edition text + kritik: München 2013, S. 54-71, hier S. 67. Vgl. zu Edison Morton Jr., Daniel L.: Sound Recording. The Life Story of a Technology, The John Hopkins University Press: Baltimore/MD 200, S. 8.

89 Vgl. Bundesverband Musikindustrie: Musikindustrie in Zahlen 2017, Berlin 2018, www.musikindustrie.de/fileadmin/bvmi/upload/02_Markt-Bestseller/Mi Z-Grafiken/2017/BVMI_ePaper_2017.pdf; Deutsches Musikinformationszentrum: Gesamtangebot und Neuerscheinungen von Pop- und Klassik-Tonträgern (physisch), Bonn 2017, www.miz.org/downloads/statistik/102/102_ Gesamtangebot_Pop-Klassiktontraeger_2017.pdf.

90 Vgl. Maria Eriksson/Rasmus Fleischer/Anna Johansson/Pelle Snickars/Patrick Vonderau: Spotify Teardown. Inside the Black Box of Streaming Music, MIT Press: Cambridge/MA 2019, S. 96. Vgl. des Weiteren z.B. https://www.cnet. $\mathrm{com} /$ news/spotify-vs-apple-music-which-is-the-best-music-service-comparedcomparison/; https://www.androidcentral.com/youtube-music-vs-spotify; https ://www.digitaltrends.com/music/apple-music-vs-spotify/.

91 Vgl. Felix Oberholzer-Gee/Koleman Strumpf: »File Sharing and Copyright«, in: Innovation Policy and the Economy 10/1 (2010), S. 19-55, hier S. 20; Deutsches Musikinformationszentrum: Gesamtangebot und Neuerscheinungen von Pop- und Klassik-Tonträgern (physisch), Bonn 2017, www.miz.org/down loads/statistik/102/102_Gesamtangebot_PopKlassiktontraeger_2017.pdf.

92 Peter Gülke: »Die Verjährung der Meisterwerke. Überlegungen zu einer Theorie der musikalischen Interpretation«, in: ders.: Auftakte - Nachspiele. Studien zur musikalischen Interpretation, Metzler/Bärenreiter: Stuttgart/Kassel 2006, S. 181-192, hier S. 181.

93 Vgl. Albrecht Riethmüller: ") She Speaks, Yet She Says Nothing: What of That?^. Romeo and Juliet bei Hector Berlioz und Leonard Bernstein «, in: Albrecht Riethmüller: Annäherung an Musik. Studien und Essays, hrsg. von Insa Bernds/Michael Custodis/Frank Hentschel, Franz Steiner Verlag: Stuttgart 2007, S. 140-154.

94 Vgl. die Aussagen von Ben Ratliff im Podcast Jon Caramanica: »Popcast: A Send-Off for Ben Ratliff«, in: The New York Times (5. August 2016), https:// www.nytimes.com/2016/08/05/arts/music/popcast-a-send-off-for-ben-ratliff.h tml.

95 Vgl. Òscar Celma: Music Recommendation and Discovery. The Long Tail, Long Fail, and Long Play in the Digital Music Space, Springer: Heidelberg 2010; Anja Nylund Hagen: »The Playlist Experience: Personal Playlists in 
Music Streaming Services«, in: Popular Music and Society 38/5 (2015), S. 625-645; Peter Knees/Markus Schedl: Music Similarity and Retrieval. An Introduction to Audio- and Web-based Strategies, Springer: Berlin 2016; Kieran Fenby-Hulse, »Rethinking the Digital Playlist: Mixtapes, Nostalgia, and Emotionally Durable Design«, in: Networked Music Cultures. Contemporary Approaches, Emerging Issues, hrsg. von Raphaël Nowak/Andrew Whelan, London 2016, S. 171-188; Bastian Lange: »The Evolution of Music Tastemakers in the Digital Age: The Rise of Algorithms and the Response of Journalists«, in: The Production and Consumption of Music in the Digital Age, hrsg. von Brian J. Hracs/Michael Seman/Tarek E. Virani, Routledge: New York 2016, S. 237-247; Dietmar Jannach/Geoffray Bonnin: »Music Recommendation«, in: Music Data Analysis. Foundations and Applications, hrsg. von Claus Weihs/ Dietmar Jannach/Igor Vatolkin/Günter Rudolph, CRC Press: New York 2017, S. 563-588; Maria Eriksson/Anna Johansson: »)Keep Smiling! «: Time, Functionality and Intimacy in Spotify's Featured Playlists«, in: Cultural Analysis 16/1 (2018), S. 68-84; Sofia Johansson/Ann Werner/Patrik Åker/Gregory Goldenzwaig: Streaming Music. Practices, Media, Cultures, Routledge: New York 2018; Maria Eriksson/Rasmus Fleischer/Anna Johansson/Pelle Snickars/ Patrick Vonderau: Spotify Teardown. Inside the Black Box of Streaming Music, MIT Press: Cambridge/MA 2019.

96 Gerhard Lauer: »Die Vermessung der Kultur. Geisteswissenschaften als Digital Humanities«, in: Big Data. Das neue Versprechen der Allwissenheit, hrsg. von Heinrich Geiselberger/Tobias Moorstedt, Suhrkamp: Berlin 2013, S. 99116, hier S. 110.

97 Vgl. Frédéric Döhl: »Potential und Risiken des Archival Turns in den Digital Humanities für die Musikwissenschaft«, in: Archiv für Musikwissenschaft 75/4 (2018), S. 301-320; Frédéric Döhl: »Digital Humanities und Bibliotheken. Über technisch-organisatorische Infrastruktur hinausgedacht«, in: ZfBB - Zeitschrift für Bibliothekswesen und Bibliographie 66/1 (2019), S. 4-18.

98 Dieser Abschnitt zur Verfasstheit der Digital Humanities ist übernommen aus Frédéric Döhl: »Potential und Risiken des Archival Turns in den Digital Humanities für die Musikwissenschaft«, in: Archiv für Musikwissenschaft 75/4 (2018), S. 301-320, hier S. 307-311.

99 Mareike König: »Was sind Digital Humanities? Definitionsfragen und Praxisbeispiel aus der Geschichtswissenschaft«, in: Digital Humanities am DHIP, 2016, https://dhdhi.hypotheses.org/2642. 
100 Vgl. Susan Schreibmann/Ray Siemens/John Unsworth: »Preface«, in: A New Companion to Digital Humanities, hrsg. von dies., Wiley Blackwell: Malden/ MA 2016, S. xvii-xviii, hier S. xvii.

101 Vgl. https://whatisdigitalhumanities.com/; Matthew Gold/Lauren F. Klein: »Digital Humanities: The Expanded Field«, in: Debates in the Digital Humanities, hrsg. von dies., 2016, http://dhdebates.gc.cuny.edu/debates/text/51; Mareike König: »Was sind Digital Humanities? Definitionsfragen und Praxisbeispiel aus der Geschichtswissenschaft«, in: Digital Humanities am DHIP, 2016, https://dhdhi.hypotheses.org/2642.

102 Julie Thompson Klein: Interdisciplining Digital Humanities: Boundary Work in an Emerging Field, University of Michigan Press: Ann Arbor/MI 2015, https://www.press.umich.edu/2525468/interdisciplining_digital_humanities.

103 Für eine Einführung in die Vorgeschichte der Digital Humanities, vgl. Julie Thompson Klein: Interdisciplining Digital Humanities: Boundary Work in an Emerging Field, University of Michigan Press: Ann Arbor/MI 2015, https ://www.press.umich.edu/2525468/interdisciplining_digital_humanities, Kap. 1 und 2; Manfred Thaller: »Geschichte der Digital Humanities« und »Digital Humanities als Wissenschaft«, in: Digital Humanities. Eine Einführung, hrsg. von Fotis Jannidis/Hubertus Kohle/Malte Rehbein, J.B. Metzler: Stuttgart 2017, S. 3-18.

104 Open access hier: http://www.digitalhumanities.org/companion/. Eine zweite Ausgabe, welche die Entwicklungen in den anderthalb Jahrzehnten dazwischen reflektiert, erschien 2016.

105 Vgl. https://www.musicology.org/networks/sg/digital-musicology.

106 Vgl. John Ashley Burgoyne/Ichiro Fujinaga/J. Stephen Downie: »Music Information Retrieval«, in: A New Companion to Digital Humanities, hrsg. von Susan Schreibmann/Ray Siemens/John Unsworth, Wiley Blackwell: Malden/ MA 2016, S. 213-227, hier S. 215.

107 Einen guten Überblick über den Stand der Dinge bis 2011 in Deutschland gibt die vom CCeH hrsg. Broschüre Digitale Geisteswissenschaften, https://dighum.de/sites/dig-hum.de/files/cceh_broschuereweb.pdf.

108 Adam Kirsch: »Technology Is Taking Over English Departments. The False Promise of the Digital Humanities«, in: The New Republic (2.5.2014), https:// newrepublic.com/article/117428/limits-digital-humanities-adam-kirsch.

109 Vgl. BMBF: Newsletter August 2018, www.research-in-germany.org/en/info service/newsletter/newsletter-2018/august-2018/digital-humanities--the-rise-of -a-new-academic-discipline.html. 
110 Vgl. Patrick Sahle: Zur Professionalisierung der Digital Humanities, https:// dhd-blog.org/?p=6174.

111 Vgl. Stephen Robertson: »The Differences between Digital Humanities and Digital History«, in: Debates in the Digital Humanities, hrsg. von Matthew Gold/Lauren F. Klein, 2016, http://dhdebates.gc.cuny.edu/debates/text/76.

112 Vgl. Camilla Leathem/Domink Adrian: Survey and Analysis of Basis Social Science and Humanities research at the Science Academies and Related Research Organisations in Europe (SASSH), ALLEA: Berlin 2015, https:// edoc.bbaw.de/frontdoor/index/index/docId/2331; DFG (GRF): http://gepris.df g.de/gepris/OCTOPUS, Suchbegriff »Digital Humanities«.

113 So wurde es z.B. im Rahmen des Akademientag 2018 für die Akademienförderung postuliert.

114 Vgl. etwa die Hochschulen in Bamberg, Bern, Bielefeld, Darmstadt, Erlangen, Gießen, Göttingen, Graz, Hamburg, Köln, Leipzig Lüneburg, Mainz, München, Passau, Regensburg, Saarbrücken, Stuttgart, Trier, Tübingen, Wuppertal, Würzburg und Zürich.

115 Vgl. http://www.gcdh.de/en/; http://cceh.uni-koeln.de/; http://dch.phil-fak.unikoeln.de/; http://kompetenzzentrum.uni-trier.de/de/; https://informationsmodel lierung.uni-graz.at/.

116 Vgl. https://www.hsu-hh.de/kdh/; http://tasaom.iwr.uni-heidelberg.de:8080/; http://mainzed.org/de/; https://www.uni-muenster.de/news/view.php?cmdid=8 993; https://www.uni-stuttgart.de/forschung/verbuende/digital-humanities/; http://kallimachos.de/kallimachos/index.php/Hauptseite.

117 Vgl. http://www.ifdhberlin.de/; https://www.saw-leipzig.de/de/ueber-die-akade mie/arbeitsgruppen/ag-digital-humanities-mitteldeutschland.

118 Vgl. https://www.listserv.dfn.de/sympa/subscribe/digmus; http://das.american studies.de/; https://www.historikerverband.de/arbeitsgruppen/ag-digitale-gw.ht ml; http://digitale-kunstgeschichte.de/; https://www.mediaevistenverband.de/di gitale-mediaevistik/; http://deutscher-romanistenverband.de/der-drv/ag-digitale -romanistik/; http://www.semiotik.eu/Digital-humanities. Die AG Germanistik im digitalen Zeitalter im Deutschen Germanistenverband hat keine Webpräsenz. Fotis Jannidis ist ein möglicher Ansprechpartner hier.

119 Vgl. https://www.allea.org/working-groups/overview/working-group-e-human ities/; https://www.akademienunion.de/arbeitsgruppen/ehumanities/ag-ehuma nities/; https://libereurope.eu/strategy/digital-skills-services/digitalhumanities/; https://www.vdb-online.org/kommissionen/forschungsnahe-dienste/. 
120 Vgl. http://www.digitalhumanities.org/dhq/; https://www.digitalstudies.org/; http://journalofdigitalhumanities.org/; https://dhcommons.org/; http://www.zf dg.de/; https://academic.oup.com/dsh.

121 Vgl. nur Dariah-DE (Hrsg.): Doing Digital Humanities - A DARIAH Bibliography, https://www.zotero.org/groups/113737/doing_digital_humanities_-_a_ dariah_bibliography?

122 Für einen Einstieg und Überblick, vgl. Anne Burdick/Johanna Drucker/Peter Lunenfeld/Todd Presner/Jeffrey Schnapp: Digital_Humanities, MIT-Press: Cambridge/MA 2012; Manfred Thaller (Hrsg.): »Controversies around the Digital Humanities«, in: Historical Social Research 37/3 (2012), https://www. gesis.org/hsr/volltext-archiv/2012/373-digital-humanities/; Melissa Terras/Julianne Nyhan/Edward Vanhoutte (Hrsg.): Defining Digital Humanities. A Reader, Ashgate: Farnham 2013; Susanne Kurz: Digital Humanities. Grundlagen und Technologien für die Praxis, 2. Auflg., Springer: Wiesbaden 2016; Matthew Gold/Lauren F. Klein (Hrsg.): Debates in the Digital Humanities, 2016, http://dhdebates.gc.cuny.edu/debates/text/51; Susan Schreibmann/Ray Siemens/John Unsworth (Hrsg.): A New Companion to Digital Humanities, Wiley Blackwell: Malden/MA 2016; Fotis Jannidis/Hubertus Kohle/Malte Rehbein: Digital Humanities. Eine Einführung, J.B. Metzler: Stuttgart 2017.

123 Vgl. Frédéric Döhl: »Potential und Risiken des Archival Turns in den Digital Humanities für die Musikwissenschaft«, in: Archiv für Musikwissenschaft 75/4 (2018), S. 301-320.

124 Vgl. für die Musikwissenschaft so zuletzt etwa Christiane Wiesenfeldt: "Zu viele Noten? Digital Humanities 2/6«, in: Frankfurter Allgemeine Zeitung (10.5.2018), http://www.faz.net/aktuell/feuilleton/hoch-schule/digital-humaniti es-eine-bilanz-2-6-musikwissenschaft-15579191.html.

125 Ramón Reichert: »Einführung«, in: Big Data. Analysen zum digitalen Wandel von Wissen, Macht und Ökonomie, hrsg. von ders., transcript: Bielefeld 2014, S. 9-31, hier S. 13, 20.

126 Vgl. Frédéric Döhl: »Potential und Risiken des Archival Turns in den Digital Humanities für die Musikwissenschaft«, in: Archiv für Musikwissenschaft 75/4 (2018), S. 301-320.

127 Fotis Jannidis: »Digitale Literaturwissenschaft«, in: Digitalität. Theorien und Praktiken des Digitalen in den Geisteswissenschaften, https://digigeist.hypo theses.org/114. Vgl. ebenso Gerhard Lauer: »Die Vermessung der Kultur. Geisteswissenschaften als Digital Humanities«, in: Big Data. Das nеие Versprechen der Allwissenheit, hrsg. von Heinrich Geiselberger/Tobias Moor- 
stedt, Suhrkamp: Berlin 2013, S. 99-116, hier S. 109f; Malte Rehbein: »Geschichtsforschung im digitalen Raum. Über die Notwendigkeit der Digital Humanities als historische Grund- und Transferwissenschaft«, in: Papstgeschichte im digitalen Zeitalter. Neue Zugangsweisen zu einer Kulturgeschichte Europas, hrsg. von Klaus Herbers/Viktoria Trenkle, Böhlau Verlag: Köln 2018, S. 19-44; Malte Rehbein: ")L'historien de demain sera programmeur ou il ne sera pas.<(Digitale) Geschichtswissenschaften heute und morgen, in: Digital Classics Online 4/1 (2018), https://journals.ub.uni-heidelberg.de/index. $\mathrm{php} / \mathrm{dco} /$ article/view/48491/41870.

128 Vgl. stellvertretend zum Bereich Konzerte und Konzertprogramm Barbara Wiermann: »musiconn.performance - Musikalische Ereignisdaten im Fachinformationsdienst Musikwissenschaft «, in: Kooperative Informationsstrukturen als Chance und Herausforderung, hrsg. von Achim Bonte/Juliane Rehnolt, De Gruyter: Berlin 2018, S. 398-415.

129 Zitat aus der Erinnerung von Lauers Keynote Lecture. Die Aussage war zugespitzt, Lauers Position ist tatsächlich vermittelnder, vgl. Gerhard Lauer: »Die Vermessung der Kultur. Geisteswissenschaften als Digital Humanities«, in: Big Data. Das neue Versprechen der Allwissenheit, hrsg. von Heinrich Geiselberger/Tobias Moorstedt, Suhrkamp: Berlin 2013, S. 99-116.

130 Vgl. Bruno Latour: »Why Has Critique Run Out of Steam? From Matters of Fact to Matters of Concern«, in: Critical Inquiry 30/2 (2004), S. 225-248.

131 Frank Hentschel: »Über Wertung, Kanon und Musikwissenschaft«, in: Der Kanon der Musik. Theorie und Geschichte. Ein Handbuch, hrsg. von Klaus Pietschmann/Melanie Wald-Fuhrmann, edition text + kritik: München 2013, S. 72-85, hier S. 75.

132 Vgl. im direkten Kontrast zueinander z.B. die benachbart erschienenen Aufsätze Frank Hentschel: »Über Wertung, Kanon und Musikwissenschaft«, in: Der Kanon der Musik. Theorie und Geschichte. Ein Handbuch, hrsg. von Klaus Pietschmann/Melanie Wald-Fuhrmann, edition text + kritik: München 2013, S. 72-85, hier S.79, 81; Michael Walter: »Kanonbildung durch begründetes Werturteil in der Musikwissenschaft«, in: ebd., S. 86-100, S. 93.

133 Vgl. für eine Diskussion genau solcher Positionen Malte Rehbein: »>L'historien de demain sera programmeur ou il ne sera pas.`(Digitale) Geschichtswissenschaften heute und morgen, in: Digital Classics Online 4/1 (2018), https://journals.ub.uni-heidelberg.de/index.php/dco/article/view/48491/41870.

134 Ben Ratliff: Every Song Ever. Twenty Ways to Listen to Music Now, Allen Lane: London 2016, S. 3f., 10. 
135 Ted Underwood: Why Everyone Should Welcome the Coming Controversy over Digital Humanities, 2011, https://tedunderwood.com/2011/01/21/a-fewreflections-on-things-that-arent-digital/.

136 Lawrence Kramer: The Hum of the World. A Philosophy of Listening, University of California Press: Berkeley/CA 2019, S. 14. Vgl. ausführlich Lawrence Kramer: Interpreting Music, University of California Press: Berkeley/CA 2011, S. 50-62.

137 Paul Guyer: A History of Modern Aesthetics, Bd. 1, Cambridge University Press: Cambridge 2014, S. 9-11, 25f., 34.

138 Das Misstrauen der Emotionsforschung gegen jede Form des Selbstberichts teile ich nicht. Vgl. hierzu Patrik N. Juslin: »Music and Emotion: Seven Questions, Seven Answers«, in: Music and the Mind. Essays in Honour of John Sloboda, hrsg. von Irène Deliège/Jane W. Davidson, Oxford University Press: New York/Oxford 2011, S. 113-135, hier S. 114-116.

139 Bruno Latour: »Why Has Critique Run Out of Steam? From Matters of Fact to Matters of Concern«, in: Critical Inquiry 30/2 (2004), S. 225-248, hier S. 232.

140 Vgl. Ludwig Wittgenstein: Philosophische Untersuchungen, Suhrkamp: Frankfurt am Main 1999, S. 313 (= Nr. 144 a.E.).

141 Ebd.

142 Vgl. nur die im zweiten Kapitel aufgegriffenen Argumente aus Joseph Kerman: »How We Got into Analysis, and How to Get Out«, in: Critical Inquiry 7/2 (1980), S. 311-331; Joseph Kerman: Contemplating Music: Challenges to Musicology, Harvard University Press: Cambridge/MA 1985; Albrecht Riethmüller: »Fremdsprache Musik«, in: Albrecht Riethmüller: Annäherung an Musik. Studien und Essays, hrsg. von Insa Bernds/Michael Custodis/Frank Hentschel, Franz Steiner Verlag: Stuttgart 2007, S. 255-268; Lawrence Kramer: Interpreting Music, University of California Press: Berkeley/CA 2011; Nicholas Cook: Beyond the Score. Music as Performance, Oxford University Press: New York/Oxford 2013; Lawrence Kramer: The Thought of Music, University of California Press: Berkeley/CA 2016.

143 Vgl. Frédéric Döhl: »Potential und Risiken des Archival Turns in den Digital Humanities für die Musikwissenschaft«, in: Archiv für Musikwissenschaft 75/4 (2018), S. 301-320; Frédéric Döhl: »Digital Humanities und Bibliotheken. Über technisch-organisatorische Infrastruktur hinausgedacht«, in: ZfBB - Zeitschrift für Bibliothekswesen und Bibliographie 66/1 (2019), S. 4-18. 
144 Frank Hentschel: Bürgerliche Ideologie und Musik. Politik der Musikgeschichtsschreibung in Deutschland 1776-1871, Campus: Frankfurt am Main 2006, S. 11.

145 Nicholas Cook/Mark Everist: »Preface«, in: Rethinking Music, hrsg. von diess., Oxford University Press: New York/Oxford 1999, S. v-xii, hier S. v.

146 Walter Feilchenfeldt: Vincent van Gogh. The Years in France. Complete Paintings 1886-1890, Philip Wilson: London 2013, S. 138.

147 Endstation Sehnsucht [1951], Warner Bros. DVD 1000052297, 2006.

148 The Royal Ballet: Romeo and Juliet [1966], Kultur DVD D1183, 1999.

149 F. Scott Fitzgerald: The Great Gatsby [1925], Broadview Press: Peterborough 2007, S. 142.

150 Vgl. Basil Smallman: The Piano Quartet and Quintet. Style, Structure, and Scoring, Clarendon Press: Oxford 1994.

151 Vgl. Wilhelm Altmann: Handbuch für Klavierquintettspieler. Wegweiser durch die Klavierquintette, Verlag für musikalische Kultur und Wissenschaft: Wolfenbüttel 1936.

152 Vgl. Hayden White: Metahistory. Die historisch Einbildungskraft im 19. Jahrhundert in Europa [1973], 2. Auflg., Fischer: Frankfurt am Main 2015.

153 Carl Dahlhaus: »Was ist Musikgeschichte?«, in: Europäische Musikgeschichte, Bd. 1, hrsg. von Sabine Ehrmann-Herfort/Ludwig Finscher/Giselher Schubert, Bärenreiter/Metzler: Kassel/Stuttgart 2002, S. 59-79, hier S. 65.

154 Bruno Latour: »Why Has Critique Run Out of Steam? From Matters of Fact to Matters of Concern«, in: Critical Inquiry 30/2 (2004), S. 225-248. Hier zitiert nach der an diese Stelle instruktiv erläuternden ausführlicheren Übersetzung von Heinz Jatho als Elend der Kritik. Vom Krieg um Fakten zu Dingen von Belang, Diaphanes: Zürich 2007. Latour spricht im Original nur von »matters of concern«.

155 Vgl. Martin Seel: Ästhetik des Erscheinens, Suhrkamp: Frankfurt am Main 2003, S. 43.

156 Den Streit in der Forschung, ob es sich nun um Gefühle, Stimmungen, eine spezielle Art ästhetischer Gefühle oder gar keine wirkliche Emotion, sondern z.B. eine Erfahrung von An- und Entspannung handelt, lasse ich hier außen vor, da jedenfalls für mein eigenes ästhetisches Erleben, um das es hier geht, die Frage klar im ersten Sinne beantwortet ist. Vgl. Patrick G. Hunter/E. Glenn Schellenberg: »Introduction to the Study of Music and Emotion«, in: Music Perception, hrsg. von Mari Riess Jones/Richard R. Fay/Arthur N. Popper, Springer: New York 2010, S. 129-164, hier S. 129-137; Patrik N. Juslin: 
»Emotional Reactions to Music«, in: The Oxford Handbook of Music Psychology, hrsg. von Susan Hallam/Ian Cross/Michael Thaut, 2. Auflg., Oxford University Press: New York/Oxford 2016, S. 197-214, hier S. 199 f.

157 Vgl. Albrecht Wellmer: Versuch über Musik und Sprache, Carl Hanser: München 2009, S. 9. Kursivierung gemäß Vorlage.

158 Vgl. Martin Seel: Ästhetik des Erscheinens, Suhrkamp: Frankfurt am Main 2003, S. 43.

159 Vgl. Joseph Kerman: »How We Got into Analysis, and How to Get Out«, in: Critical Inquiry 7/2 (1980), S. 311-331; Joseph Kerman: Contemplating Music: Challenges to Musicology, Harvard University Press: Cambridge/MA 1985, S. 17.

160 Richard Taruskin: »Foreword«, in: Lydia Goehr: The Imaginary Museum of Musical Works, 2. Auflg., Oxford University Press: Oxford 2007, S. viii.

161 Noël Carroll: On Criticism, Routledge: New York 2009, S. 14.

162 Elijah Wald: How the Beatles Destroyed Rock 'n' Roll. An Alternative History of American Popular Music, Oxford University Press: New York/Oxford 2009, S. 8.

163 Paul Guyer: A History of Modern Aesthetics, Bd. 1, Cambridge University Press: Cambridge 2014, S. 9-11, 25f., 34.

164 Ebd., S. 10.

165 Für meine Zwecke jedenfalls. In der Ästhetik ist die Begriffsgeschichte durchaus komplex, wie Guyer anmerkt. Vgl. ebd., S. 9.

166 Vgl. Theodor W. Adorno: »Schöne Stellen« [1965], in: Theodor W. Adorno, Gesammelte Schriften Bd. 18., Musikalische Schriften V, hrsg. von Rolf Tiedemann, Suhrkamp: Frankfurt am Main 2003, S. 695-718, insb. S. 700.

167 Vgl. Arthur C. Danto, »Embodiment, Art History, Theodicy, and the Abuse of Beauty: A Response to My Critics«, in: Inquiry 48/2 (2005), S. 189-200, hier S. 190f.

168 David Hume: »On the Standard of Taste« [1757], in: David Hume. Essays. Moral, Political, and Literary, hrsg. von T. H. Green/T. H. Grose, Scientia: Aalen 1992, S. 266-284, hier S. 268f.

169 Darauf wird noch zurückzukommen sein. Vgl. einführend zu dem Unterschied Patrik N. Juslin/John A. Sloboda: Handbook of Music and Emotion. Theory, Research, Applications, Oxford University Press: New York/Oxford 2010; Peter Rinderle: Die Expressivität von Musik, Mentis: Paderborn 2010; Patrik N. Juslin: »Music and Emotion: Seven Questions, Seven Answers«, in: Music and the Mind. Essays in Honour of John Sloboda, hrsg. von Irène Deliège/Jane W. 
Davidson, Oxford University Press: New York/Oxford 2011, S. 113-135; Susan Hallam/Ian Cross/Michael Thaut (Hrsg.): The Oxford Handbook of Music Psychology, 2. Auflg., Oxford University Press: New York/Oxford 2016; Stefan Zwinggi: Musik als affektive Selbstverständigung. Eine integrative Untersuchung über musikalische Expressivität, Karl Aber: Freiburg im Breisgau 2016; Alexander Wilfing: »>Autonomania< und `Ideology of Autonomy<. Die Autonomie-Diskussion in der analytischen Musikästhetik und der New Musicology«, in: Von der Autonomie des Klangs zur Heteronomie der Musik. Musikwissenschaftliche Antworten auf Musikphilosophie, hrsg. von Nikolaus Urbanek/Melanie Wald-Fuhrmann, J.B. Metzler: Stuttgart 2018, S. 179-210.

170 Vgl. Hans Ulrich Gumbrecht: »Epiphanien«, in: Dimension ästhetischer Erfahrung, hrsg. von Joachim Küpper/Christoph Menke, Suhrkamp: Frankfurt am Main 2003, S. 203-222.

171 Ben Ratliff: Every Song Ever. Twenty Ways to Listen to Music Now, Allen Lane: London 2016, S. 228. Kursivierung hinzugefügt.

172 Heinz Schlaffer: »Wolfgang Braungart/Joachim Jacob, Stellen, schöne Stellen. Oder: Wo das Verstehen beginnt (Besprechung)«, in: Arbitrium 32/1 (2014), S. 10-12, hier S. 11. Vgl. auch Theodor W. Adorno: »Schöne Stellen« [1965], in: Theodor W. Adorno, Gesammelte Schriften Bd. 18., Musikalische Schriften $V$, hrsg. von Rolf Tiedemann, Suhrkamp: Frankfurt am Main 2003, S. 695718, hier S. 700.

173 Vgl. Hans Ulrich Gumbrecht: »Epiphanien«, in: Dimension ästhetischer Erfahrung, hrsg. von Joachim Küpper/Christoph Menke, Suhrkamp: Frankfurt am Main 2003, S. 203-222.

174 Stefan Majetschak: Ästhetik. Eine Einführung, Junius: Hamburg 22010, S. 48.

175 Martin Seel: Ästhetik des Erscheinens, Suhrkamp: Frankfurt am Main 2003, S. 190.

176 Paul Guyer: A History of Modern Aesthetics, Bd. 1, Cambridge University Press: Cambridge 2014, S. 25f., 34.

177 Vgl. Nicholas Cook: Beyond the Score. Music as Performance, Oxford University Press: New York/Oxford 2013, S. 32, 56-90.

178 Joseph Kerman: Contemplating Music: Challenges to Musicology, Harvard University Press: Cambridge/MA 1985, S. 18f.

179 Susan McClary: »Writing about Music - and the Music of Writing«, in: The Future of Scholarly Writing. Critical Interventions, hrsg. von Angelika Bammer/Ruth-Ellen Boetcher Joeres, Palgrave Macmillan: Basingstoke 2015, S. 205-214, hier S. 206. 
180 Albrecht Riethmüller: »Musikästhetik und musikalischer Genuss«, in: $\mathrm{Al}$ brecht Riethmüller: Annäherung an Musik. Studien und Essays, hrsg. von Insa Bernds/Michael Custodis/Frank Hentschel, Franz Steiner Verlag: Stuttgart 2007, S. 155-190, hier S. 189.

181 Albrecht Riethmüller: »Erkaltete Gefühle. Wie in den Diskursen über Musik die Emotionalität unter die Räder kam«, in: ders.: Lost in Music. Essays zur Perspektivierung von Urteil und Erfahrung, Franz Steiner Verlag: Stuttgart 2015, S. 198-203, hier S. 198.

182 Frank Hentschel: Bürgerliche Ideologie und Musik. Politik der Musikgeschichtsschreibung in Deutschland 1776-1871, Campus: Frankfurt am Main 2006, S. 11.

183 Theodor W. Adorno: Ästhetische Theorie (= Theodor W. Adorno. Gesammelte Schriften, Bd. 7, hrsg. von Rolf Tiedemann), Suhrkamp: Frankfurt am Main 2003, S. 449.

184 Michel-Rolph Trouillot: Silencing the Past: Power and the Production of History, Beacon Press: Boston 1995, S. 26.

185 Friedrich Geiger/Tobias Janz: »Ökonomie und Kanon«, in: Der Kanon der Musik. Theorie und Geschichte. Ein Handbuch, hrsg. von Klaus Pietschmann/ Melanie Wald-Fuhrmann, edition text + kritik: München 2013, S. 858-888, hier S. 862f.

186 Vgl. Eberhard Ortland: »Ästhetik als Wissenschaft der sinnlichen Erkenntnis«, in: Deutsche Zeitschrift für Philosophie 49/2 (2001), S. 257-274; Ursula Franke: »Sinnliche Erkenntnis - was sie ist und was sie soll. A.G. Baumgartens Ästhetik-Projekt zwischen Kunstphilosophie und Anthropologie«, in: Aufklärung 20 (2008), S. 73-99.

187 Martin Seel: Ästhetik des Erscheinens, Suhrkamp: Frankfurt am Main 2003, S. $16 f$.

188 Ben Ratliff: Every Song Ever. Twenty Ways to Listen to Music Now, Allen Lane: London 2016, S. 228.

189 Vgl. Stefan Majetschak: Ästhetik. Eine Einführung, Junius: Hamburg 22010, S. 41-51.

190 A. O. Scott: Better Living Through Criticism. How to Think about Art, Pleasure, Beauty, and Truth, Jonathan Cape: London 2016, S. 52, 54.

191 Ebd., S. 80.

192 Ramón Reichert: »Digital Humanities«, in: Handbuch Medienwissenschaft, hrsg. von Jens Schröter, J.B. Metzler: Stuttgart 2014, S. 511-515, hier S. 514. 
193 Vgl. einführend Frédéric Döhl: »Potential und Risiken des Archival Turns in den Digital Humanities für die Musikwissenschaft«, in: Archiv für Musikwissenschaft 75/4 (2018), S. 301-320; Frédéric Döhl: »Digital Humanities und Bibliotheken. Über technisch-organisatorische Infrastruktur hinausgedacht«, in: ZfBB - Zeitschrift für Bibliothekswesen und Bibliographie 66/1 (2019), S. 4-18.

194 Ramón Reichert: »Digital Humanities«, in: Handbuch Medienwissenschaft, hrsg. von Jens Schröter, J.B. Metzler: Stuttgart 2014, S. 511-515, hier S. 514.

195 Julie Thompson Klein: Interdisciplining Digital Humanities: Boundary Work in an Emerging Field, Michigan University Press: Ann Arbor/MI 2015, Kap. 2, https://www.press.umich.edu/2525468/interdisciplining_digital_humanities.

196 Bruno Nettl: »Contemplating Ethnomusicology: What Have We Learned?«, in: Archiv für Musikwissenschaft 67/3 (2010), S. 173-186, hier S. 184.

197 Ludwig Wittgenstein: Philosophische Untersuchungen, Suhrkamp: Frankfurt am Main 1999, S. 313 (= Nr. 144 a.E.).

198 Vgl. zur Streitbarkeit Melanie Wald-Fuhrmann: »Musik und Subjektivität«, in: Historische Musikwissenschaft. Grundlagen und Perspektiven, hrsg. von Michele Calella/Nikolaus Urbanek, J.B. Metzler: Stuttgart 2013, S. 289-306.

199 Vgl. Elijah Wald: How the Beatles Destroyed Rock 'n' Roll. An Alternative History of American Popular Music, Oxford University Press: New York/ Oxford 2009, S. 10.

200 Lawrence Kramer: The Hum of the World. A Philosophy of Listening, University of California Press: Berkeley/CA 2019, S. 14.

201 Vgl. Frank Hentschel, Bürgerliche Ideologie und Musik. Politik der Musikgeschichtsschreibung in Deutschland 1776-1871, Campus: Frankfurt am Main 2006.

202 Susan Sontag: »Against Interpretation«, in: Against Interpretation and Other Essays, 6. Auflg., Dell: New York 1966, S. 13f. Abs. vor »What« im Original.

203 Gerhard Schulze: Die Erlebnisgesellschaft. Kultursoziologie der Gegenwart, Campus: Frankfurt am Main 1992, S. 67.

204 Lawrence Kramer: The Hum of the World. A Philosophy of Listening, University of California Press: Berkeley/CA 2019, S. 13.

205 Vgl. Georg W. Bertram: Kunst als menschliche Praxis. Eine Ästhetik, Suhrkamp: Berlin 2014, S. 13, 19.

206 Vgl. Stefan Zwinggi: Musik als affektive Selbstverständigung. Eine integrative Untersuchung über musikalische Expressivität, Karl Aber: Freiburg im Breisgau 2016. 
207 Vgl. Matthias Vogel: »Music that changed my life. Pop-Musik und Selbstverständnis« (2018) in: Pop weiter denken. Neue Anstöße aus Jazz Studies, Philosophie, Musiktheorie und Geschichte, hrsg. von Ralf von Appen/André Doehring, transcript: Bielefeld 2018, S. 211-228.

208 Ben Ratliff: Every Song Ever. Twenty Ways to Listen to Music Now, Allen Lane: London 2016, S. 3f., 10.

209 Vgl. https://www.youtube.com/watch?v=CmNUhNHG-_Y.

210 Vgl. Nina Polaschegg: Populäre Klassik - Klassik populär: Hörerstrukturen und Verbreitungsmedien im Wandel, Böhlau: Köln 2005. Vgl. auch Jörn Peter Hiekel (Hrsg.): Populär vs. elitär. Wertvorstellungen und Popularisierungen der Musik heute, Schott: Mainz 2013; Christian Kellersmann: »Die >neue Klassik««, in: Das Konzert II. Beiträge zum Forschungsfeld der Concert Studies, hrsg. von Martin Tröndle, transcript: Bielefeld 2018, S. 379-387 (erweiterte Fassung von Christian Kellersmann: »Wege der Erneuerung«, in: Das Konzert I. Neue Aufführungskonzepte für eine klassische Form, 2. Auflg., hrsg. von Martin Tröndle, transcript: Bielefeld 2011, S. 221-226); Marius Carboni: »The Classical Music Business«, in: The Music Industry Handbook, 2. Auflg. hrsg. von Paul Rutter, Routledge: New York 2016, S. 195-223; Julia Haferkorn: »Dancing to Another Tune: Classical Music in Nightclubs and Other Non-Traditional Venues«, in: The Classical Music Industry, hrsg. von Chris Dromey/Julia Haferkorn, Routledge: New York 2018, S. 148-171. Primäre Ressource für Informationen über die Neoklassik sind bislang aber musikjournalistische Beiträge, Interviews sowie Rezensionen von Konzerten und Alben (vgl. nur die vielen Kritiken auf Popseiten wie pitchfork.com), aber auch allgemeinere Berichte wie Selim Bulut: »Just Don't Call It \Indie Classical««, in: The Guardian (1.11.2013), https://www.theguardian.com/music/musicblog/20 13/nov/01/neo-classical-nils-frahm; Tobias Ruderer: »Die neueste Form der Gebrauchsmusik nennt sich Neoklassik. Spur einer Regression«, in: VANMagazin (29.7.2015), https://van.atavist.com/neoklassik; Bettina Jech: »NeoClassical: The Unexciting Mixture «, in: Goethe-Institut Australien (2016), https:/www.goethe.de/ins/au/en/kul/mag/20771217.html; Hartmut Welchser: »Wo Genres nicht mehr gedehnt oder ausgetrickst werden, sondern einfach gesprengt, entsteht meistens ziemlich langweilige Musik«, VAN-Magazin (1.2. 2017), https://van.atavist.com/genreloses-feelgood; Michael Hann: »Neoclassical Superstars: Leaders of a Gentle Revolution«, in: Financial Times (4.7. 2018), https://www.ft.com/content/402bb948-7de9-11e8-af48-190d103e32a4; Anna Codrea-Rado: »Is It Classical, or Pop? Nils Frahm Is Worried, but Not 
About That «, in: The New York Times (1.2.2018), https://www.nytimes.com/ 2018/02/01/arts/music/nils-frahm.html; Jesper Klein: »Zwischen den Stilen«, in: niusic.de (5.2.2019), https://www.niusic.de/artikel/reportage-pascal-schum acher (unter Verweis auf eine Konzertreihe der Philharmonie Luxemburg in der Spielzeit 2018/19 mit Vertretern der Neoklassik wie Helbig, Arnalds und Gonzales). Man vgl. auch die Reihe »Neue Meister« beim großen Indielabel Edel Germany (https://neue-meister-music.com/de/), die Christian Kellersmann aufgebaut hat, der zuvor bei Universal Classics vergleichbare Initiativen wie Yellow Lounge oder die Reecomposed-Reihe initiierte und seit Anfang 2019 für das Themengebiet bei BMG zuständig ist.

211 Vgl. William Robin: »The Rise and Fall of >Indie Classical«: Tracing a Controversial Term in Twenty-First Century New Music«, in: Journal of the Society for American Music 12/1 (2018), S. 55-88.

$212 \mathrm{Zu} \mathrm{Brahms,} \mathrm{Sørensen} \mathrm{und} \mathrm{Say,} \mathrm{vgl.} \mathrm{die} \mathrm{Besprechungen} \mathrm{später.}$

Im Fall von John Alden Carpenter war sein 1934 komponiertes Klavierquintett sein letztes vollendest größeres Kammermusikwerk. Ein hörenswertes Stück, vgl. 2002, Naxos 8.559103 (Paul Posnak, Vega Quartet). In Carpenters Fall nahm die Musik die interessante Wendung hin zu einer Sinfonie, Carpenters II. In den Jahren 1946/47 überarbeitete er beide Varianten. Eine dritte, unvollständig gebliebene Revision des Klavierquintetts findet sich obendrein in den Manuskripten Carpenters. Vgl. an Literatur zu Komponist und Werk Gregory Burnside Pike: The Three Versions of the Quintet for Piano and Strings by John Alden Carpenter [Diss.], University of Miami: Miami 1981; Joan O'Connor: John Alden Carpenter. A Bio-Bibliography, Greenwood Press: Westport/CT 1994; Howard Pollack: Skyscraper Lullaby: The Life and Music of John Alden Carpenter, Smithsonian Institute Press: Washington D.C. 1995 = John Alden Carpenter. A Chicago Composer, University of Illinois Press: Urbana 2001.

Im Fall von Henze steht sein dreisätziges Klavierquintett aus den frühen 1990er Jahren im Kontext von Trauermusik. Insgesamt hat das Klavierquintett mit den drei Sätzen Con Ferocia - Adagio - Litania über weite Strecken einen vergleichsweise sphärischen Charakter, vgl. 1999, Philips 4467102 (Peter Serkin, Guarneri Quartet - Uraufführungsbesetzung, Ersteinspielung). Die oft einstimmigen Linien des Klaviers im Register der Violinen sorgen für ein auffallend hohes Maß an Verschmelzung der Ensembleteile. Jedoch ist es die Ekstase des Schlusses, die den stärksten Eindruck auf mich hinterlässt. Wiederholt setzt die Musik an und bricht wieder ab. Um plötzliche in einer 
hochenergetische Motorik überzugehen. Wie wenn ein Auto Gas gibt und immer schneller wird. Die Umgebung erscheint immer verschwommener. Die Intensität wächst. Doch plötzlich, völlig unvermittelt, bricht die Musik ab. Kein Verlangsamung. Kein Aufprall. Nichts als Nachhall bleibt. Und völlige Irritation. Die Arbeit entstand parallel zu Henzes Requiem. In diesem Fall erschöpft sich die Beziehung jedoch nicht wie so oft in zeitlicher Koinzidenz oder recht oberflächlichen Ähnlichkeiten im Ausdruck. Henzes Requiem, im gleichen Jahr wie das Klavierquintett uraufgeführt, sind, wie bereits der Untertitel anmerkt, »Neun geistliche Konzerte für Klavier solo, konzertierende Trompete und großes Kammerorchester«. Die drei Sätze des Klavierquintetts sind zugleich Vorstudien und Variationen der Sätze II-IV dieses Requiem. Die Distanz ist jedoch hoch, schon aufgrund der Größe seines Orchesterapparats und den damit einhergehenden, ungleich größeren Differenzierungsmöglichkeiten in der Orchestration, von denen Henzes vielfarbige Partitur auch reich gebraucht macht. Vgl. an Literatur zu Komponist und Werk Peter Petersen: Hans Werner Henze. Werke der Jahre 1984-1993, Schott: Mainz 1995; Hans-Klaus Jungheinrich (Hrsg.): Im Laufe der Zeit - Kontinuität und Veränderung bei Hans Werner Henze, Schott: Mainz 2002; Jens Rosteck: Hans Werner Henze. Rosen und Revolution. Eine Biographie, Propyläen: Berlin 2009; Norbert Abels/Elisabeth Schmierer (Hrsg.): Hans Werner Henze und seine Zeit, Laaber: Laaber 2013; Stephen C. Dowes: After Mahler. Britten, Weill, Henze, and Romantic Redemption, Cambridge University Press: Cambridge 2013.

Im Fall von Gourzis Vibrato 1 und Vibrato 2, geschrieben für die Festspiele Europäische Wochen Passau 2010 und in deren Rahmen von Siegfried Mauser und dem Rodin Quartett uraufgeführt, handelt es sich um hypnotische Studien von gut 8 Minuten Aufführungsdauer. So nah aufeinander bezogen, dass die Komponistin in einer nicht alltäglichen Werknotiz anrät, sie nicht direkt hintereinander zu spielen. Vibrato 2 wirkt dabei wie ein freier gehaltener Kommentar auf Vibrato 1. Die Streichquartette spielen in beiden Varianten dasselbe. Das Klavier hat aber in Vibrato 2 Wahlmöglichkeiten, wann und wie oft es seinen Part spielt, ganz frei in seinem Tempo. Anders als in Vibrato 1. Vibrato 1 schreitet langsam, aber unerbittlich im 5/4-Metrum voran. Eine immer gleiche Basslinie, vorgestellt in Klavier (linke Hand) und Cello, kreist und kreist: $\mathrm{e}-\mathrm{f}-\mathrm{c}-\mathrm{a}-\mathrm{h}$. Das bleibt auch in Vibrato 2. Konsonante Musik. Dissonanz als Farbe. Diatonische Sekundreibungen vor allem zu Beginn. Aber nicht als Hauptsache. Bewusst leise Musik. Mit vielen kleinen Details. Und einem be- 
eindruckend desorientierenden Effekt des 5/4-Metrum, das vordergründig doch so gleichmäßig voranschreitet. Man achte z.B. auf den Effekt, den es in der Referenzaufnahme macht, wenn Vibrato 1 als Ende einfach abbricht, in der hier aufgenommenen Version von Vibrato 2 aber an derselben Stelle des Streichquartettsatzes nicht aufhört zu spielen. Sondern noch ein wenig nachsinnt. Vgl. 2014, ECM 2309 (Lorenda Ramou, Ensemble Coriolis). Vgl. an Literatur zu Komponistin und Werk Steffen Ramlow/Hannes Ravic: Der Taktstock: Dirigenten erzählen von ihrem Instrument, Zsolnay: Wien 2001, S. 116118; Anon.: »11 Fragen an Konstantia Gourzi«, in: Neue Musikzeitung 56/3 (2007), https://www.nmz.de/artikel/11-fragen-an-konstantia-gourzi; Hans-Dieter Grünefeld: »Aus Allem Eins und aus Einem Alles - die Komponistin Konstantia Gourzi«, in: Musik \& Theater 6 (2013), S. 33-35, https://web.archive. org/web/20161029193047/http://www.konstantiagourzi.com/content/33_35_g ourzi.pdf; Christoph Schlüren: Konstantia Gourzi: Ein biographischer Essay, 2016, https://repertoire-explorer.musikmph.de/de/konstantia-gourzi/ (Verlagsseite).

213 Uraufführung: München, 7. Januar 1980. Referenznoten: Berlin: Ries \& Erler, 2002. Aufnahmen: 1993, Timpani 1C1018 (François Kerdoncuff, Quatuor Sine Nomine); 1996, Bayer Records BR 100269 (Daniele Bellik, Quatuor Élyséen - Aufnahme von 1979); 2004, Tacet 119 (Clarens Quintet) = 2017, Tacet B 119; 2004, Faculty WFFC-0601 (Erwin Stein, Michael Arlt, Albena Danailova, Ruth Elena Schindel, Dietrich von Kaltenborn).

214 Vgl. 2004, Tacet 119 (Clarens Quintet), 47.27-47.37 min.

215 Roman Brotbeck: »Alles sehr allmählich: Anmerkungen zum Komponisten Wilhelm Furtwängler«, in: Wilhelm Furtwängler in Diskussion, hrsg. von Chris Walton, Amadeus: Winterthur 1996, S. 41-55, hier S. 41.

216 Elisabeth Furtwängler/Günter Birkner (Hrsg.): Wilhelm Furtwängler. Aufzeichnungen 1924-1954, Brockhaus: Wiesbaden 1980, S. 275. Vgl. des Weiteren insb. Roman Brotbeck: »Alles sehr allmählich: Anmerkungen zum Komponisten Wilhelm Furtwängler«, in: Wilhelm Furtwängler in Diskussion, hrsg. von Chris Walton, Amadeus: Winterthur 1996, S. 41-55; Günter Birkner: Furtwänglers Selbstverständnis als Komponist, in: Furtwängler-Studien I, hrsg. von Sebastian Krahnert, Ries \& Erler: Berlin 1998, S. 36-45; Bruno d'Heudières: „Wilhelm Furtwängler als Komponist - das Ethos eines Künstlers«, in: Furtwängler-Studien I, hrsg. von Sebastian Krahnert, Ries \& Erler: Berlin 1998, S. 71-95; Roman Brotbeck: »Der dirigierende Komponist. Anmerkungen zu Furtwänglers kompositorischem Werk«, in: Zwischen Skylla 
und Charybdis. Wilhelm Furtwängler im Brennpunkt, hrsg. von der Stiftung Lucerne Festival, Lucerne Festival: Luzern 2004, S. 75-82; Norbert Meurs: »Dirigieren oder Komponieren? Der merkwürdige Zwiespalt des Wilhelm Furtwängler«, in: Musikgeschichten - Vermittlungsformen, hrsg. von Martina Bick/Julia Heimerdinger/Krista Warnke, Böhlau: Köln 2010, S. 147-158; Wolfgang Auhagen: »Wilhelm Furtwängler: ein Dirigent des `Espressivo《«, in: Ereignis und Exegese: Musikalische Interpretation, Interpretation der Musik, hrsg. von Camilla Bork/Tobias Robert Klein/Burkhard Meischein/ Andreas Meyer/Tobias Plebuch, Ed. Argus: Schliengen 2011, S. 54-63; Chris Walton: »Wilhelm Furtwängler and the Return of the Muse«, in: ders., Lies and Epihanies. Composers and Their Inspiration from Wagner to Berg, University of Rochester Press: Rochester/NY 2014, S. 94-109 [dt. 2017]; John Knight: »Prolific Composers and Transcribers in Spite of Active Conducting: Felix Weingartner, Wilhelm Furtwängler, and Leopold Stokowski«, in: Dirigieren und Komponieren, hrsg. von Alexander Drčar/Wolfgang Gratzer, Rombach: Freiburg im Breisgau 2017, S. 99-130.

217 Haruki Murakami: Wovon ich rede, wenn ich vom Laufen rede, DuMont: Köln 2008, S. 6.

218 Vgl. Elijah Wald: How the Beatles Destroyed Rock 'n' Roll. An Alternative History of American Popular Music, Oxford University Press: New York/ Oxford 2009; Elijah Wald: »Forbidden Sounds: Exploring the Silences of Music History«, in: Geschichte wird gemacht. Zur Historiographie populärer Musik, hrsg. von Dietrich Helms/Thomas Phleps, transcript: Bielefeld 2014, S. 25-39.

219 Vgl. Pierre Bourdieu: »Ökonomisches Kapital, kulturelles Kapital, soziales Kapital«, in: Soziale Ungleichheiten, hrsg. von Reinhard Kreckel, Schwartz: Göttingen 1983, S. 183-198.

220 Vgl. Patrik N. Juslin: »Music and Emotion: Seven Questions, Seven Answers«, in: Music and the Mind. Essays in Honour of John Sloboda, hrsg. von Irène Deliège/Jane W. Davidson, Oxford University Press: New York/Oxford 2011, S. 113-135, hier S. 114-116.

221 Theodor W. Adorno: Ästhetische Theorie (= Theodor W. Adorno. Gesammelte Schriften, Bd. 7, hrsg. von Rolf Tiedemann), Suhrkamp: Frankfurt am Main 2003, S. 449.

222 Vgl. Patrik N. Juslin/John A. Sloboda: Handbook of Music and Emotion. Theory, Research, Applications, Oxford University Press: New York/Oxford 2010; Susan Hallam/Ian Cross/Michael Thaut (Hrsg.): The Oxford Handbook 
of Music Psychology, hrsg. von, 2. Auflg., Oxford University Press: New York/Oxford 2016.

223 Trotz Adornos Vorwurf des Dilettantismus, wenn man nicht zugleich über die Musik als Ganzes reflektiere. Vgl. Theodor W. Adorno: Ästhetische Theorie (= Theodor W. Adorno. Gesammelte Schriften, Bd. 7, hrsg. von Rolf Tiedemann), Suhrkamp: Frankfurt am Main 2003, S. 449.

224 Albrecht Riethmüller: »Fremdsprache Musik«, in: Albrecht Riethmüller: Annäherung an Musik. Studien und Essays, hrsg. von Insa Bernds/Michael Custodis/Frank Hentschel, Franz Steiner Verlag: Stuttgart 2007, S. 255-268, hier S. 268.

225 Vgl. einführend zum Kuratorischen Paul O’Neill (Hrsg.): Curating Subjects, Open Ed.: London 2007; Paul O’Neill: »The Curatorial Turn: From Practice to Discourse «, in: Issues in Curating Contemporary Art and Performance, hrsg. von Judith Rugg/Michèle Sedgwick, Intellect: Bristol 2007, S. 13-28; Hans Ulrich Obrist: A Brief History of Curating, JRP Ringier Kunstverlag: Zürich 2009; Sheena Iyengar: The Art of Choosing, Grand Central: New York 2010; Beatrice von Bismarck/Jörn Schafaff/Thomas Weski (Hrsg.): Cultures of the Curatorial, Sternberg Press: Berlin 2012; Paul O'Neill: The Culture of Curating and the Curating of Culture(s), MIT Press: Cambridge/MA 2012.

226 Hans Ulrich Gumbrecht: »Was am 〉Kuratieren« nervt«, in: Digital/Pausen. FAZ Blogs (3.8.2012), https://blogs.faz.net/digital/2012/08/03/warum-kuratier en-nervt-80/.

227 Dorothea von Hantelmann: »Affluence and Choice. The Social Significance of the Curatorial«, in: Cultures of the Curatorial, hrsg. von Beatrice von Bismarck/Jörn Schafaff/Thomas Weski, Sternberg Press: Berlin 2012, S. 41-51, hier S. 47.

228 Nicht, dass es in diesen Forschungsbereichen allzu viele Anschlussmöglichkeiten zu meinem Thema hier gäbe, vgl. Dean Keith Simonton: »Emotion and Composition in Classical Music: Historiometric Perspectives«, in: Handbook of Music and Emotion. Theory, Research, Applications, hrsg. von Patrik N. Juslin/John A. Sloboda, Oxford University Press: New York/Oxford 2010, S. 347-366, hier S. 362; Patrik N. Juslin: »Emotional Reactions to Music«, in: The Oxford Handbook of Music Psychology, hrsg. von Susan Hallam/Ian Cross/Michael Thaut, 2. Auflg., Oxford University Press: New York/Oxford 2016, S. 197-214, hier 201.

229 Vgl. William K. Wimsatt/Monroe C. Beardsley: »Der intentionale Fehlschluss «, Roland Barthes: »Der Tod des Autors« und Michel Foucault: »Was 
ist ein Autor?«, in: Texte zur Theorie der Autorschaft, hrsg. von Fotis Jannidis/Gerhard Lauer/Matias Martinez/Simone Winko, Reclam: Stuttgart 2000, S. 84-104, 185-193, 198-229.

$230 »$ In most academic disciplines, the premium put on objectivity [...]«, wie es bei McClary heißt. Vgl. Susan McClary: »Writing about Music - and the Music of Writing «, in: The Future of Scholarly Writing. Critical Interventions, hrsg. von Angelika Bammer/Ruth-Ellen Boetcher Joeres, Palgrave Macmillan: Basingstoke 2015, S. 205-214, hier S. 205. "And if it is the nature of much music theory to de-emphasise both individual agency and history, then the same is true of music psychology«, wie es bei Nicholas Cook heißt. Vgl. Nicholas Cook: Beyond the Score. Music as Performance, Oxford University Press: New York/Oxford 2013, S. 61.

231 Vgl. Robert Christgau: »Writing about Music is Writing First«, in: Popular Music 24/3 (2005), S. 415-421, hier S. $415 f$.

232 Vgl. David Howes/Constance Classen: Ways of Sensing. Understanding the Senses in Society, Routledge: New York 2014, S. 11-13; David Howes: »Law's Sensorium: On the Media of Law and the evidence of the Senses in Historical and Cross-Cultural Perspective«, in: Sensing Law, hrsg. von Sheryl N. Hamilton/Diana Majury/Dawn Moore/Neil Sargent/Christiane Wilke, Routledge: New York 2017, S. 53-72, hier S. 55f. Vgl. zu den hieran anschließenden mal musiknahen, mal musikfernen Themen und Schwerpunkten der Sound Studies einführend Holger Schulze (Hrsg.): Sound Studies: Traditionen - Methoden - Desiderate. Eine Einführung, transcript: Bielefeld 2008; Trevor Pinch/Karin Bijsterveld (Hrsg.): The Oxford Handbook of Sound Studies, Oxford University Press: New York/Oxford 2012; Andi Schoon/Axel Volmar (Hrsg.): Das geschulte Ohr. Eine Kulturgeschichte der Sonifikation, transcript: Bielefeld 2012; Jonathan Sterne (Hrsg.): The Sound Studies Reader, Routledge: New York 2012; Axel Volmar/Jens Schröter (Hrsg.): Auditive Medienkulturen. Techniken des Hörens und Praktiken der Klanggestaltung, transcript: Bielefeld 2013; Jens Gerrit Papenburg/Holger Schulze (Hrsg.): Sound as Popular Culture A Research Companion, MIT Press: Cambridge/MA 2016; Michael Bull (Hrsg.): The Routledge Companion to Sound Studies, Routledge: New York 2019.

233 Maurice Merleau-Ponty: Phänomenologie der Wahrnehmung, De Gruyter: München 1974, S. 7.

234 Vgl. Bruno Latour: »Why Has Critique Run Out of Steam? From Matters of Fact to Matters of Concern«, in: Critical Inquiry 30/2 (2004), S. 225-248. 
235 Lydia Goehr: The Imaginary Museum of Musical Works, 2. Auflg., Oxford University Press: New York/Oxford 2007, S. 174.

236 Vgl. Nicholas Cook: Beyond the Score. Music as Performance, Oxford University Press: New York/Oxford 2013, S. 6; Nicholas Cook: Music as Creative Practice, Oxford University Press: New York/Oxford 2018, S. 49.

237 Vgl. Christoph Conrad: »Die Dynamik der Wenden. Von der neuen Sozialgeschichte zum cultural turn«, in: Geschichte und Gesellschaft 22 [Sonderheft] (2006), S. 133-160; Doris Bachmann-Medick: Cultural Turns. New Orientations in the Study of Culture, De Gruyter: Berlin 2016.

238 Wolfgang Braungart/Joachim Jacob: Stellen, schöne Stellen. Oder: Wo das Verstehen beginnt, Wallstein: Göttingen 2012, S. 7, 9.

239 Vgl. Martin Seel: Ästhetik des Erscheinens, Suhrkamp: Frankfurt am Main 2003, S. 190.

240 Vgl. Lawrence Kramer: Interpreting Music, University of California Press: Berkeley/CA 2011, S. 46-62.

241 Jerrold Levinson: Musical Concerns. Essays in Philosophy of Music, Oxford University Press: New York/Oxford 2015, S. 25f.

242 Jerrold Levinson: Music, Art, and Metaphysics. Essays in Philosophical Aesthetics, Oxford University Press: New York/Oxford 2011, S. 333f.

243 Vgl. für einen einführenden Überblick Patrik N. Juslin/John A. Sloboda: Handbook of Music and Emotion. Theory, Research, Applications, Oxford University Press: New York/Oxford 2010; Peter Rinderle: Die Expressivität von Musik, Mentis: Paderborn 2010; Susan Hallam/Ian Cross/Michael Thaut (Hrsg.): The Oxford Handbook of Music Psychology, 2. Auflg., Oxford University Press: New York/Oxford 2016; Stefan Zwinggi: Musik als affektive Selbstverständigung. Eine integrative Untersuchung über musikalische Expressivität, Karl Aber: Freiburg im Breisgau 2016; Alexander Wilfing: »>Autonomania und `Ideology of Autonomy<. Die Autonomie-Diskussion in der analytischen Musikästhetik und der New Musicology«, in: Von der Autonomie des Klangs zur Heteronomie der Musik. Musikwissenschaftliche Antworten auf Musikphilosophie, hrsg. von Nikolaus Urbanek/Melanie Wald-Fuhrmann, J.B. Metzler: Stuttgart 2018, S. 179-210.

244 Vgl. Patrik N. Juslin: »Music and Emotion: Seven Questions, Seven Answers«, in: Music and the Mind. Essays in Honour of John Sloboda, hrsg. von Irène Deliège/Jane W. Davidson, Oxford University Press: New York/Oxford 2011, S. 113-135. 
245 Vgl. Vladimir J. Konečni: »Music, Affect, Method, Data: Reflections on the Carroll Versus Kivy Debate«, in: American Journal of Psychology 126/2 (2013), S. 179-195.

246 Vgl. Susan McClary: »Writing about Music - and the Music of Writing«, in: The Future of Scholarly Writing. Critical Interventions, hrsg. von Angelika Bammer/Ruth-Ellen Boetcher Joeres, Palgrave Macmillan: Basingstoke 2015, S. 205-214, hier S. 205.

247 Die Herkunft des Zitats scheint unklar, vgl. http://www.78s.ch/2010/01/07/drpop-von-wem-stammt-das-zitat- $\% \mathrm{E} 2 \% 80 \% 9 \mathrm{Cwriting}$-about-music-is-like-dan cing-about-architecture/.

248 Vgl. Lawrence Kramer: The Thought of Music, University of California Press: Berkeley/CA 2016, S. 46; Robert Christgau: »Writing about Music is Writing First«, in: Popular Music 24/3 (2005), S. 415-421, hier S. 415.

249 Albrecht Riethmüller: »Fremdsprache Musik«, in: Albrecht Riethmüller: Annäherung an Musik. Studien und Essays, hrsg. von Insa Bernds/Michael Custodis/Frank Hentschel, Franz Steiner Verlag: Stuttgart 2007, S. 255-268, hier S. 265.

250 Vgl. Nelson Goodman: Sprachen der Kunst [1968], Suhrkamp: Frankfurt am Main 1997.

251 Albrecht Riethmüller: »Fremdsprache Musik«, in: Albrecht Riethmüller: Annäherung an Musik. Studien und Essays, hrsg. von Insa Bernds/Michael Custodis/Frank Hentschel, Franz Steiner Verlag: Stuttgart 2007, S. 255-268, hier S. 266

252 Albrecht Riethmüller: »Fremdsprache Musik«, in: Albrecht Riethmüller: Annäherung an Musik. Studien und Essays, hrsg. von Insa Bernds/Michael Custodis/Frank Hentschel, Franz Steiner Verlag: Stuttgart 2007, S. 255-268, hier S. 264.

253 Robert DiYanni: »Sound and Sense. Writing about Music«, in: Journal of Basic Writing 2/4 (1980), S. 62-71, hier S. 62.

254 Vgl. stellv. nur zuletzt Stephen A. Crist/Roberta Montemorra Marvin (Hrsg.): Historical Musicology: Sources, Methods, Interpretations, University of Rochester Press: Rochester/NY 2004; J. P. E. Harper-Scott/Jim Samson (Hrsg.): An Introduction to Music Studies, Cambridge University Press: Cambridge 2009; Nicole Schwindt-Gross: Musikwissenschaftliches Arbeiten. Hilfsmittel - Techniken - Aufgaben, Bärenreiter: Kassel 2010; Burkhard Meischein: Einführung in die historische Musikwissenschaft, Dohr: Köln 2011; Marcus S. Kleiner/ Michael Rappe (Hrsg.): Methoden der Populärkulturforschung, LIT Verlag: 
Münster 2012; Kordula Knaus/Andrea Zedler (Hrsg.): Musikwissenschaft studieren. Arbeitstechnische und methodische Grundlagen, Herbert Utz: München 2012; Laurie Sampsel: Music Research: A Handbook, Oxford University Press: New York 22013; Matthew Gardner/Sara Springfeld, Musikwissenschaftliches Arbeiten. Eine Einführung, Bärenreiter: Kassel 2014; Jan Hemming: Methoden der Erforschung populärer Musik, Springer VS: Wiesbaden 2016 .

255 Vgl. stellv. nur Robert DiYanni: »Sound and Sense. Writing about Music«, in: Journal of Basic Writing 2/4 (1980), S. 62-71; Mark A. Radice: Irvine's Writing about Music, 3. Auflg., Amadeus Press: New York 1999; Patricia J. Flowers: »What Was That? - Talking about What We Hear in Music«, in: Applications of Research in Music Education 21/2 (2002), S. 42-51; James R. Cowdery: How to Write About Music. The RILM Manual of Style, RILM: New York 2006; Jonathan Bellman: A Short Guide to Writing about Music, 2. Auflg., Person Longman: New York 2007; Richard J. Wingell: Writing about Music. An Introductory Guide, 4. Auflg., Prentice Hall: Upper Saddle River/NJ 2009; Thomas Donahue: A Style and Usage Guide to Writing about Music, Scarecrow Press: Lanham/MD 2010; Trevor Herbert: Music in Words. A Guide to Researching and Writing about Music, 2. Auflg., ABRSM: London 2012; Martina Krause-Benz/Lars Oberhaus (Hrsg.): Musik und Gefühl. Interdisziplinäre Annäherungen in musikpädagogischer Perspektive, Olms: Hildesheim 2012; D. Kern Holoman: Writing about Music. A Style Sheet, University of California Press: Berkeley/CA ${ }^{32014}$; Susan McClary: »Writing about Music - and the Music of Writing«, in: The Future of Scholarly Writing. Critical Interventions, hrsg. von Angelika Bammer/Ruth-Ellen Boetcher Joeres, Palgrave Macmillan: Basingstoke 2015, S. 205-214; Marc Woodworth/Ally-Jane Grossan (Hrsg.): How to Write about Music. Excerpts from the 33 1/3 Series, Magazines, Books and Blogs with Advice from Industry-Leading Writers, Bloomsbury: New York 2015. Vgl. auch z.B. Rebekka Hüttmann: Wege der Vermittlung von Musik. Ein Konzept auf der Grundlage allgemeiner Gestaltungsprinzipien, Wißner: Augsburg 2009; Wolfgang Rüdiger (Hrsg.): Musikvermittlung - wozu? Umrisse und Perspektiven eines jungen Arbeitsfeldes, Schott: Mainz 2014.

256 Vgl. Lawrence Kramer: Interpreting Music, University of California Press: Berkeley/CA 2011, S. 13-17; Lawrence Kramer: The Thought of Music, University of California Press: Berkeley/CA 2016, S. 23-64. 
257 Vgl. nur an jüngeren Studien stellv. zuletzt Gunter Reus: »Musikjournalismus - Ergebnisse aus der wissenschaftlichen Forschung «, in: Wissenschaftliche Perspektiven auf Musik und Medien, hrsg. von Stefan Weinacht/Helmut Scherer, Wiesbaden 2008, S. 85-102; Till Krause/Stefan Weinacht: »Musikzeitschriften«, in: Handbuch Musik und Medien, hrsg. von Holger Schramm, Konstanz 2009, S. 329-361; Gunter Reus: »Musikjournalismus in der Zeitung «, in: Handbuch Musik und Medien, hrsg. von Holger Schramm, Konstanz 2009, S. 299-328; Chris Atton: »Writing About Listening: Alternative Discourses in Rock Journalism«, in: Popular Music 28/1 (2010), S. 53-67; Òscar Celma: Music Recommendation and Discovery. The Long Tail, Long Fail, and Long Play in the Digital Music Space, Springer: Heidelberg 2010; Don McLeese: »Straddling the Cultural Chasm: The Great Divide between Music Criticism and Popular Consumption «, in: Popular Music and Society 33/4 (2010), S. 433-447; André Doehring: Musikkommunikatoren: Berufsrollen, Organisationsstrukturen und Handlungsspielräume im Popmusikjournalismus, transcript: Bielefeld 2011; Christoph Griessner: »Journalistische Qualität von Musikkritiken. Eine inhaltsanalytische Untersuchung unter Berücksichtigung subkultureller Funktionen von Popjournalismus«, in: Weißbuch Kulturjournalismus, hrsg. von Wolfgang Lamprecht, Wien 2012, S. 563-573; Paula Hearsum: »Music Journalism«, in: Specialist Journalism, hrsg. von Barry Turner/ Richard Orange, New York 2013, S. 107-123; Christoph Jacke: Einführung in Populäre Musik und Medien, LIT Verlag: Berlin 22013, insb. S. 115-131; Chris Atton: „Curating Popular Music. Authority and History, Aesthetics and Technology«, in: Popular Music 33/3 (2014), S. 413-427; Gert Keunen: »The Social Process of Second-Order Selection«, in: ders., Alternative Mainstream. Making Choices in Pop Music, Amsterdam 2014, S. 311-362; Gunter Reus/ Teresa Naab: »Verhalten optimistisch. Wie Musikjournalistinnen und Musikjournalisten ihre Arbeit, ihr Publikum und ihre Zukunft sehen - eine Bestandsaufnahme«, in: Publizistik, 59/2 (2014), S. 107-133; Marco Frei: »Klassikfeuilleton«, in: Kulturjournalismus. Portraits \& Perspektiven, hrsg. von Petra Weber, Berlin 2015, S. 251-265; Anja Nylund Hagen: »The Playlist Experience: Personal Playlists in Music Streaming Services«, in: Popular Music and Society 38/5 (2015), S. 625-645; Morten Michelsen: »Music Criticism and Taste Culture«, in: The Routledge Reader in the Sociology of Music, hrsg. von John Sheperd/Kyle Devine, New York 2015, S. 211-220; Jeremy Wade Morris/Devon Powers: „Control, Curation and Musical Experience in Streaming Music Services«, in: Creative Industries Journal 8/2 (2015), S. 106-122; 
Simon Warner, »In Print and On Screen: The Changing Character of Popular Music Journalism«, in: The SAGE Handbook of Popular Music, hrsg. von Andy Bennett/Steve Waksman, Thousand Oaks/CA 2015, S. 439-455; Chris Atton: »Writing about Popular Music: Continuity, Change and Convergence in a Digital Age«, in: Simon Obert/Fritz Trümpi (Hrsg.): Musikkritik: Historische Zugänge und systematische Perspektiven, Mille Tre: Wien 2015, S. 11-32; Kieran Fenby-Hulse, »Rethinking the Digital Playlist: Mixtapes, Nostalgia, and Emotionally Durable Design«, in: Networked Music Cultures. Contemporary Approaches, Emerging Issues, hrsg. von Raphaël Nowak/Andrew Whelan, Palgrave Macmillan: London 2016, S. 171-188; Charles Lindsay: »An Exploration Into How the Rise of Curation Within Streaming Services Has Impacted How Music Fans in the UK Discover New Music«, in: Journal of Promotional Communications 4/1 (2016), S. 115-141; Dietmar Jannach/Geoffray Bonnin, »Music Recommendation«, in: Music Data Analysis. Foundations and Applications, hrsg. von Claus Weihs/Dietmar Jannach/Igor Vatolkin/ Günter Rudolph, New York 2017, S. 563-588; Joachim Mischke: »Musikjournalismus für alle - zwischen Qualität und Quote?«, in: Studien zum 250. Todestag Johann Matthesons. Musikschriftstellerei und -journalismus in Hamburg, hrsg. von Simon Kannenberg, Weidler: Berlin 2017, S. 207-230; Gunter Reus/Ruth Müller-Lindenberg (Hrsg.): Die Notengeber. Gespräche mit Journalisten über die Zukunft des Musikkritik, Springer: Wiesbaden 2017; Gunter Reus: »Musikjournalismus in Zeitungen und Blogs«, in: Handbuch Musik und Medien, 2. Auflg., hrsg. von Holger Schramm, Springer: Wiesbaden 2019 (im Druck).

258 Susan Holtfreter: Die Musikkritik im Wandel. Eine soziologisch-textlinguistische Untersuchung, Peter Lang: Frankfurt am Main 2013, S. 166f. Vgl. auch Robert D. Schick: Classical Music Criticism, Routledge: New York 1996; Marc Woodworth/Ally-Jane Grossan (Hrsg.): How to Write about Music. Excerpts from the 33 1/3 Series, Magazines, Books and Blogs with Advice from Industry-Leading Writers, Bloomsbury: New York 2015.

259 Vgl. Hans Heinrich Eggebrecht/Albrecht Riethmüller (Hrsg.): Handwörterbuch der musikalischen Terminologie, Franz Steiner Verlag: Wiesbaden/Stuttgart 1992-2005, https://www.sim.spk-berlin.de/handwoerterbuch_der_musika lischen_terminologie_1742.html; D. Kern Holoman: Writing about Music. A Style Sheet, 3. Auflg., University of California Press: Berkeley/CA 2014. 
260 Vgl. Max Haas: „Warum schreibt man über Musik?«, in: Historische Musikwissenschaft. Grundlagen und Perspektiven, hrsg. von Michele Calella/Nikolaus Urbanek, J.B. Metzler: Stuttgart 2013, S. 26-34.

261 Vgl. Peter Benary: Klangrede und Wortlaut. Zum Verhältnis von Musik und Sprache, Chronos: Zürich 2006.

262 Vgl. stellv. nur zuletzt Enrico Fubini: Geschichte der Musikästhetik, J.B. Metzler: Stuttgart 1997, S. 204-213, 385-414; Albrecht Riethmüller (Hrsg.): Sprache und Musik. Perspektiven einer Beziehung, Laaber: Laaber 1999; Albrecht Wellmer: Versuch über Musik und Sprache, Carl Hanser: München 2009; Klaus Wolfgang Niemöller: Der sprachhafte Charakter der Musik, 2. Auflg., Dohr: Köln 2010; Christian Grüny (Hrsg.): Musik und Sprache. Dimensionen eines schwierigen Verhältnisses, Velbrück: Weilerwist 2012; Christian Utz (Hrsg.): Musik-Sprachen. Beiträge zur Sprachnähe und Sprachferne von Musik im Dialog mit Albrecht Wellmer, Pfau: Saarbrücken 2013; Diedrich Diederichsen: Über Pop-Musik, Kiepenheuer \& Witsch: Köln 2014, S. 87-114; Hartmut Krones (Hrsg.): Rhetorik und Sprache, De Gruyter: Berlin 2016.

263 Vgl. stellv. nur Gerhard Albersheim: Die Tonsprache, Schneider: Tutzing 1980; Peter Benary: Klangrede und Wortlaut. Zum Verhältnis von Musik und Sprache, Chronos: Zürich 2006.

264 Vgl. Annette Kreutziger-Herr/Winfried Bönig: Die 101 wichtigsten Fragen: Klassische Musik, C.H. Beck: München 2009, S. 131; Kathleen Marie Higgins: The Music between Us. Is Music a Universal Language?, University of Chicago Press: Chicago 2012; Stefan Zwinggi: Musik als affektive Selbstverständigung. Eine integrative Untersuchung über musikalische Expressivität, Karl Aber: Freiburg im Breisgau 2016, S. 70.

265 Diedrich Diederichsen: Über Pop-Musik, Kiepenheuer \& Witsch: Köln 2014, S. 107.

266 Vgl. Lawrence Kramer: Interpreting Music, University of California Press: Berkeley/CA 2011, S. 46.

267 Lawrence Kramer: Interpreting Music, University of California Press: Berkeley/CA 2011, S. 54. Vgl. auch Lawrence Kramer: The Hum of the World. A Philosophy of Listening, University of California Press: Berkeley/CA 2019, S. 14-16.

268 Vgl. Kai Müller: »Der Unfassbare. Zum Tod von David Bowie«, in: Der Tagesspiegel (12.1.2016), https://www.tagesspiegel.de/themen/reportage/zum -tod-von-david-bowie-der-unfassbare/12817544.html. 
269 »Nicht nur wurden durch ihn zahllose Genres wie Glam Rock, New Wave,

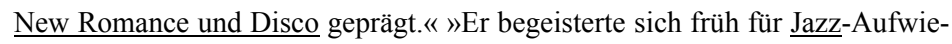
gler wie Charles Mingus und John Coltrane und spielte selbst Saxophon.« »Eines androgynen, märchenhaft-wilden Alter Ego, das Schminke und Fummel in der Rockmusik populär machte.«

270 »Er begeisterte sich früh für Jazz-Aufwiegler wie Charles Mingus und John Coltrane $[\ldots] . \ll$

271 »Klar, wurde er jetzt auch nostalgisch, wenn er sich in >Where Are We Now an den Potsdamer Platz erinnerte, auf dessen Brache er einst vom Hansa Studio aus blicken konnte, während er `Heroes « sang.«

272 Kein Anschauungsbeispiel im Müllers Text. Eine gute Übersicht zum Einstieg, auf welche Parameter sich alles achten ließe, findet sich in Robert D. Schick: Classical Music Criticism, Routledge: New York 1996, S. 141f. Eine ausführliche Vertiefung bieten Lawrence Kramer: Interpreting Music, University of California Press: Berkeley/CA 2011; Ben Ratliff: Every Song Ever. Twenty Ways to Listen to Music Now, Allen Lane: London 2016.

273 »Der deutschen Maschinen-Musik setzte Bowie mit seinen drei Alben 〉Low`, $>$ Heroes` (beide 1977) sowie >Lodger` (1979) und beraten von Mastermind Brian Eno ein elegisches Denkmal [...].«»Ohnehin suchte Bowie stets nach Gefährten, die secht` waren, die keinen Umweg brauchten für ihre Emotionen und geradeheraus auf ein Ziel zustürmten wie Lou Reed oder Iggy Pop."

274 ") Blackstar〈 ist Bowies Abschiedsgruß."

275 »Sein Körper - eine Skulptur, die Musik - lebendiger Raum.«»[...] und das alte Warhol-Credo in zeitgemäß apokalyptischer Zuspitzung - >we can be heroes, just for one day.«

$276 »$ Mit erschöpften Atemgeräuschen, stolpernden, einander überholenden Beats und der kraftlos gewordenen, geisterhaften Stimme eines Sterbenden, der noch einmal große Fragen wälzt.« »Und sein erster Hit >Space Oddity< trägt diese defensive Vorsicht in sich wie ein Wasserzeichen, das von der hymnischen Melodie erst allmählich übermalt wird.«

$277 »[\ldots]$ in die Fläche treibende Schrammelriffs der Gitarre [...].«

$278 »[\ldots]$ in die Fläche treibende Schrammelriffs der Gitarre, stupide Beats in der Endlosschleife [...].« »Musikalisch stach in den 90er Jahren nur noch `Outside` heraus, eine Musik, die auch für ein Computerspiel konzipiert war und Bowies wachsendes Interesse an undeterminierten, frei wabernden Sounds verriet.« 
279 Frank Sibley: »Ästhetische Begriffe« [1959/62], in: Das ästhetische Urteil. Beiträge zur sprachanalytischen Ästhetik, hrsg. von Rüdiger Bittner/Peter Pfaff, Kiepenheuer \& Witsch: Köln 1977, S. 87-110, hier S. 90f. Anhand der Auswertung und des Vergleichs von Fallbeispielen aus der Musikkritik ist diese theoretische Position empirisch unterlegt z.B. in Robert D. Schick: Classical Music Criticism, Routledge: New York 1996; Christiane Thim-Mabrey: Grenzen der Sprache - Möglichkeiten der Sprache: Untersuchungen zur Textsorte Musikkritik, Peter Lang: Frankfurt am Main 2001; Robert Christgau: »Writing about Music is Writing First«, in: Popular Music 24/3 (2005), S. 415-421; Susan Holtfreter: Die Musikkritik im Wandel. Eine soziologischtextlinguistische Untersuchung, Peter Lang: Frankfurt am Main 2013. Zahlreiche Anthologien von Fallbeispielen stehen überdies zur Verfügung, sich unschwer selbst an einem Vergleich zu versuchen, z.B. Jacques Barun: Pleasures of Music. An Anthology of Writing about Music and Musicians from Cellini to Bernard Shaw, The Viking Press: New York 1951; Nicolas Slonimsky: Lexicon of Musical Invective. Critical Assaults on Composers since Beethoven's Time [1953], Norton: New York 2000; Friedrich Geiger: Verdikte über Musik. Eine Dokumentation, J.B. Metzler: Stuttgart 2005 oder die Buchreiche Da Capo Press' Best Music Writing (2000-2011).

280 Frank Sibley: »Ästhetische Begriffe« [1959/62], in: Das ästhetische Urteil. Beiträge zur sprachanalytischen Ästhetik, hrsg. von Rüdiger Bittner/Peter Pfaff, Kiepenheuer \& Witsch: Köln 1977, S. 87-110, hier S. 91.

281 »Mit erschöpften Atemgeräuschen, stolpernden, einander überholenden Beats und der kraftlos gewordenen, geisterhaften Stimme eines Sterbenden, der noch einmal große Fragen wälzt.« Eine ausführliche Vertiefung zum Thema Metaphern in der Musikvermittlung findet sich in Roger W. H. Savage: Hermeneutics and Music Criticism, Routledge: New York 2010, S. 85-102; Daniel Hesselmann: In Methapern über Musik sprechen. Grundlagen zur Differenzierung metaphorischer Sprache im Musikunterricht, Dohr: Köln 2015.

282 »Es brauchte Jahre, um diese Verlegenheit abzulegen. Und sein erster Hit >Space Oddity< trägt diese defensive Vorsicht in sich wie ein Wasserzeichen, das von der hymnischen Melodie erst allmählich übermalt wird.« »Der deutschen Maschinen-Musik setzte Bowie mit seinen drei Alben >Low<, >Heroes` (beide 1977) sowie >Lodger` (1979) und beraten von Mastermind Brian Eno ein elegisches Denkmal: [...].«

283 »Mit erschöpften Atemgeräuschen, stolpernden, einander überholenden Beats und der kraftlos gewordenen, geisterhaften Stimme eines Sterbenden, der noch 
einmal große Fragen wälzt.« »Mit 〉Let's Danceく hatte Bowie 1983 abermals einen solchen Hit und tanzte im weißen Anzug eines Midnight-Macho durch die Disco.«

$284 »$ Mit dem Effekt, dass eine heitere, zutiefst depressive Musik entstand. Sie drückte einfach nur Bowies Trauer darüber aus, nicht der sein zu können, der er gerne wäre, $[\ldots] . \ll$

285 »Mit dem Effekt, dass eine heitere, zutiefst depressive Musik entstand.«

286 »Das ist die letzte Inszenierung des ewigen Verwandlungskünstlers, eine treue, versöhnliche Geste, die den edlen Charakter Bowies auch im Abgang noch einmal sichtbar macht. Wenn man auch hören kann, dass ihm dieser nicht leicht fällt. Mit schmerzhafter Dringlichkeit beklagt er, nicht `alles` geben zu können. Der Schatten senkt sich auf sein Lebenscredo: >Seeing more and feeling less / Saying No but meaning Yes / This is all I ever meant / that's the message that I sent.«

287 »Als die Popmusik ihr Heil in den Methoden suchte, derer sich Bowie lange vorher bedient hatte, wandte er sich von ihr ab. Mit seinen elektronischen Ausflügen der späten 90er gewann er kaum jemanden für sich.«

288 Man könnte den Beispieltext von Müller insgesamt so lesen. Der Text versucht vor allem, Bowies Vielseitigkeit zu vermitteln. Auf sprachlicher Ebene korrespondiert das damit, dass der Text mit ganz unterschiedlichen Stil- und Beschreibungsmitteln arbeitet. Hauptanwendungsbereich wäre aber die Ekphrasis.

289 Vgl. Robert Christgau: »Writing about Music is Writing First«, in: Popular Music 24/3 (2005), S. 415-421, hier S. $416 f$.

290 Vgl. Jacques Derrida: »Das Gesetz der Gattung« [1980], in: ders.: Gestade, Passagen-Verlag: Wien 1994, S. 245-284.

291 Für Anmerkungen und Vorschläge an dieser Stelle danke ich Mario Dunkel.

292 »Mit erschöpften Atemgeräuschen, stolpernden, einander überholenden Beats und der kraftlos gewordenen, geisterhaften Stimme eines Sterbenden, der noch einmal große Fragen wälzt.« »Denn als früherer Kunststudent faszinierte ihn die Idee des Gesamtkunstwerks. Sein Körper - eine Skulptur, die Musik lebendiger Raum. $« » E r$ kaufte sich ein großzügiges Appartement, und_da er immer weniger auf andere Musiker angewiesen sein wollte, entstanden viele seiner Alben nun am Computer."

293 »Mit erschöpften Atemgeräuschen, stolpernden, einander überholenden Beats und der kraftlos gewordenen, geisterhaften Stimme eines Sterbenden, der noch einmal große Fragen wälzt.« »Denn als früherer Kunststudent faszinierte ihn 
die Idee des Gesamtkunstwerks. Sein Körper - eine Skulptur, die Musik lebendiger Raum." »Er kaufte sich ein großzügiges Appartement, und da er immer weniger auf andere Musiker angewiesen sein wollte, entstanden viele seiner Alben nun am Computer."

294 »Bowie ließ eine exquisite Auswahl weißer Session-Musiker in Philadelphia schwarzen Soul spielen und verzichtete auf den Mumpitz der Selbstüberhöhung.«

295 »Doch das Geheimnis der Popularität findet sich nicht nur in guten Songs, konventionell genug, um von vielen Menschen gemocht zu werden, aber auch neu und so ambitioniert, dass sie dem Publikum neue Gefühle erschließen. Mit >Let's Danceく hatte Bowie 1983 abermals einen solchen Hit [...].« Sehr interessant sind an dieser Stelle die Aussagen vieler Musikjournalisten, die einen Schwerpunkt auf ihre eigene Hörposition legen, bei Popkonzerten etwa am Rand, in der Menge, vor der Bühne, tanzend oder stehend, vgl. Marc Woodworth/Ally-Jane Grossan (Hrsg.): How to Write about Music. Excerpts from the 33 1/3 Series, Magazines, Books and Blogs with Advice from IndustryLeading Writers, Bloomsbury: New York 2015, S. 45-48.

296 https://www.youtube.com/watch? $\mathrm{v}=\mathrm{Jb} 7 \mathrm{vDC} 5$ les4.

297 Alte Gesamtausgabe. Via IMSLP ist u.a. auch die Ausgabe von 1865 und das Autograph einsehbar. Das Werk ist als Teil der neuen wissenschaftlichen Brahms-Gesamtausgabe - Serie II, Bd. 4 - auch bereits in aktuellerer historisch-kritischer Fassung erschienen, vgl. München: Henle 1999. Und in vielen weiteren zeitgenössischen Ausgaben.

Soweit möglich habe ich für diese virtuelle, imaginierte Ausstellung mit Noten gearbeitet, die frei im Internet einzusehen sind. Das ist Absicht. Um einen schnellen ersten Nachvollzug zu ermöglichen. Denn darum geht es hier. Aktuelle, manchmal auch wissenschaftlich-kritische Ausgaben, sind bei Bedarf rasch recherchiert. Werke mit Kontrabass und ohne 2. Violine sind mit [*] gekennzeichnet. Das angegebene Jahr ist, soweit ermittelbar, jenes der Uraufführung, ansonsten jenes der Erstveröffentlichung, hilfsweise der Entstehung, wenn die Uraufführung und die Erstveröffentlichung sehr viel später erfolgten.

$298 \mathrm{https}: / /$ www.youtube.com/watch?v=Jb7vDC5les4.

$299 \mathrm{http}: / / \mathrm{www} . e d i t i o n s i l v e r t r u s t . c o m /$ fruhling-piano-quintet-Op.30.htm.

300 Vgl. auch Michael Staudinger (Hrsg.): Bruno Walter erinnern, Universal Edition: Wien 2013 (mit Noten des Klavierquintetts).

301 Über deutsche Bibliotheken kann man auch die Erstausgabe einsehen, vgl. Mainz: Schott 1915. 
$302 \mathrm{https} / /$ ianvenables.com/product/piano-quintet-op-27-full-score-and-parts/.

303 Vgl. auch 2008, Live at Colburn School (https://www.youtube.com/watch?v= TLOdqOhnf4Q; https://www.youtube.com/watch?v=oYmBSBZL1-w - Gloria Cheng, Calder Quartet); 2015, Signum Classics SIGCD413 (Thomas Adès, Calder Quartet); 2017, Cybele Records SACD 261603 (Dimitri Vassilakis, DoelenKwartet).

304 Vgl. auch 2007, Sello Autor SA01398 (Paula Coronas, García Abril Quartett [heute: Estarellas Quartett] - Ersteinspielung); 2007, Live at Málaga (https:// www.youtube.com/watch?v=8De-BkMjNZg - Paula Coronas, García Abril Quartett [Satz I]).

305 https://imslp.org/wiki/Piano_Quintet_No.1\%2C_Op.10_(Fries\%2C_Albin).

306 https://www.youtube.com/watch?v=Bk5Uwbc5QmQ. Vgl. auch 2014, Tzadik TZ 9007 (Sarah Rothenberg, Brentano String Quartet - Uraufführungsbesetzung).

307 https://www.newsounds.org/story/listen-jefferson-friedman-chiara-string-quart et-simone-dinnerstein/ und https://blogcritics.org/concert-review-chiara-stringquartet-and-simone-dinnerstein-piano-quintets-brahms-and-friedman/ (Simone Dinnerstein, Chiara String Quartet - Uraufführung). Vgl. auch 2015, Live at The Metropolitan Museum of Art (https://www.metmuseum.org/metmedia/vi deo/concerts/chiara-string-quartet-simone-dinnerstein - Simone Dinnerstein, Chiara String Quartet).

308 Ein sechszeiliges Gedicht, dessen Zeilen sich auf das Stück als Ganzes beziehen, dient den Sätzen zugleich als Überschrift. Ein Satz, ein Vers. Das Gedicht stammt von Margaret LeMay und wurde laut dem Programmheft zur Uraufführung in Reaktion auf Friedmans Musik geschrieben. Diese ist also keine Gedichtvertonung. Es passt jedoch nicht weniger durch diese vertauschten Rollen. Im Gegenteil: »The Heart Wakes Into: the way in - which touches | the lights of others cut into the hills. | Dawn alone, being | rent and want; | the fold of bald wings | is having, had, departed.« https://blogs.loc.gov/music/files/ 2014/12/Fine-Program-Final.12.01.2014.pdf, S. 16.

309 https://www.youtube.com/watch?v=dMxQDbQiTNU - Vgl. auch 2014, Live at Da Camera of Houston (https://www.youtube.com/watch?v=QfsIpE21mIU Vijay Iyer, Bretano Quartet - Ausschnitt [Sätze 5, 6 und 8] von der Uraufführung); 2017, Live at the Ojai Music Festival (https://www.youtube.com/wat ch?v=Xik1JQEC5kI - Vijay Iyer, Brentano Quartet).

310 Vgl. http://dig-that-lick.eecs.qmul.ac.uk/. 
311 Vgl. Marie-Agnes Dittrich: »Tradition und Innovation im Klavierquintett in fMoll op. 34«, in: Die Kammermusik von Johannes Brahms: Tradition und Innovation: Bericht über die Tagung Wien 1997, hrsg. von Gernot Gruber, Laaber: Laaber 2001, S. 175-185; Siegfried Oechsle: »Klaviertrios, Klavierquartette, Klavierquintett«, in: Brahms-Handbuch, hrsg. von Wolfgang Sandberger, J.B. Metzler: Stuttgart 2009, S. 408-436; Thomas Hauschka: »Klavierquintett f-Moll op. 34 - Sonate für zwei Klavier, op. 34bis«, in: Johannes Brahms. Interpretationen seiner Werke, Bd. 1, hrsg. von Claus Brockmaier/ Siegfried Mauser, Laaber: Laaber 2013, S. 227-233. Aufgrund der kanonischen Position von Komponist und Werk finden sich zahlreiche weitere Bemerkungen zu op. 34 in Brahms-Literatur, allg. Literatur zur Kammermusik (einschl. Konzertführern) und allg. Literatur zur Musikgeschichte des 19. Jahrhunderts.

312 »Emotional impact« für dieses säulenheilige Standardstück des Genres habe ich tatsächlich erst hier das erste Mal erfahren. In dieser Darbietung. Als ob es diese Autorität von einem Meisterwerk für mich vermenschlicht hat. Nicht nur Sockel. Nicht nur Kanon. Nicht nur Lehrbuchwissen. Hier geht es auch um etwas, dass mich angeht. Eine spannende Erfahrung, zu sehen, wie viel eine einzige Interpretation noch für einen ändern kann nach so vielen desselben Stücks, die man zuvor schon gehört hatte.

313 Smallman gibt 1870 als Entstehungsjahr an, vgl. Basil Smallman: The Piano Quartet and Quintet. Style, Structure, and Scoring, Clarendon Press: Oxford 1994, S. 74. Castillon-Spezialist Fauquet im Booklettext zur Referenzaufnahme vermerkt aber den 5. April 1864 für die Uraufführung und 10. März 1870 als Tag der Erstaufführung in Paris. Referenzliteratur zum Komponisten ist einzig Joël-Marie Fauquet: Alexis de Castillon, sa vie, son ouvre [Diss.], L'École Pratique des Hautes Études: Paris 1976.

Bazille, nur wenig jünger als Castillon, gehörte zum Kern der frühen Impressionisten, deren Entwicklung er aufgrund seiner von Haus guten ökonomischen Situation auch wirtschaftlich wesentlich förderte. Bazille freilich starb als einziger aus diesem Kreis früh, kurz vor dem Durchbruch dieser künstlerischen Bewegung als Soldat im Deutsch-Französischen Krieg von 1870/71. Auch Castillon diente und seine ohnehin fragile Gesundheit erholte sich nicht mehr von diesem Krieg. Er erlag wenig später 1873 einem Fieber. Bazille und Castillon verbinden daher nicht nur Alter, Epoche und Kriegserfahrung, die Qualität ihrer Arbeit und deren ästhetische Anmutung. Gemeinsam ist ihnen auch eine zentrale Position bei der Etablierung künstlerischer Gruppen, an deren 
zentraler Bedeutung für die Kunst- bzw. Musikproduktion des verbleibenden 19. Jahrhunderts in Frankreich ihr eigenes Schaffen aufgrund ihres frühen Todes aber kaum mehr einen Anteil haben konnte. An Bazilles einstige Position innerhalb der Gruppe, die erst nach Bazilles Tod ab ihrer ersten gemeinsam Ausstellung 1874 Impressionisten genannt werden sollte, erinnern z.B. die gegenseitigen Portraits mit Pierre-Auguste Renoir und Claude Monet, Bazilles Aufnahme in Henri Fatin-Latours Gruppenportrait Un atelier aux Batignolles (1870) neben u.a. Édouard Manet, Monet und Renoir oder Bazilles eigenes Ensemblebild L'atelier de Bazille (1870). Castillon seinerseits war wiederum nach dem Krieg Gründungssekretär der Société Nationale de Musique und stand am 17. November 1871 neben Werken u.a. von César Franck, Jules Massenet und Camille Saint-Saëns auf dem Programm des ersten Konzerts jener Vereinigung, die für gut zwei Generationen ein großer Motor für die Produktion neuer heimischer Kammermusik wurde.

314 Vgl. Ennio Speranza: "Alcuni appunti sulla musica da camera di Giovanni Sgambati: i due Quartetti per archi e i due Quintetti per archi e pianoforte«, in: La Musica di Giovanni Sgambati, hrsg. von Paola Canfora/Francescantonio Pollice, Curci: Mailand 2014, S. 87-108.

315 Vgl. Folco Perrino: Giuseppe Martucci, 4 Bd., Centro Studi Martucciani: Novara 1992-2009; Francesco Bissoli: »Il Quintetto per pianoforte e archi op. 45. Modificazioni e intenzioni compositive nell'autografo del Conservatorio di Napoli«, in: Giuseppe Martucci. Da Capua all'Accademia di Santa Cecilia, hrsg. von Antonio Rostagno, Accademia Nazionale di Santa Cecilia: Rom 2012, S. 275-294.

316 Vgl. 1992, Aura 416-2 (Patrizia Prati, Quartetto di Venezia); 1995, Dynamic 1999 (Ex Novo Ensemble); 2016, Brilliant Classics 94968 (Maria Semeraro, Quartetto Noferini).

317 Vgl. Katharine Leiska: Skandinavische Musik in Deutschland um 1900. Symphonien von Christian Sinding, Victor Bendix und Carl Nielsen zwischen Gattungstradition und Nord-Imagines, Peter Lang: Frankfurt am Main 2012.

318 Vgl. David R. Beveridge: »Dvořák’s Piano Quintet, Op. 81: The Schumann Connection«, in: Chamber Music Quarterly (1984), S. 2-10; David R. Beveridge: »Dvořák's Dumka and the Concept of Nationalism in Music Historiography«, in: Journal of Musicological Research 12/4 (1993), S. 303-325; Klaus Döge: Dvorák. Leben - Werke - Dokumente, 2. Auflg., Atlantis: Zürich 1997; Wolfgang Winterhager: „Gebrochene Akkordik als Begleitfiguration in Dvoráks Klavierkammermusik, am Beispiel von op. 81 und op. 87«, in: The Work 
of Antonín Dvoŕák (1841-1904): Aspects of Composition - Problems of Editing - Reception, hrsg. von Jarmila Gabrielová/Jan Kachlík, Akademie der Wissenschaften der Tschechischen Republik: Prag 2007, S. 111-119; Markéta Štědronská: Die Klaviermusik von Antonín Dvořák. Studien und Vergleiche mit Werken von Brahms, Schneider: Tutzing 2010. Aufgrund der kanonischen Position von Komponist und Werk finden sich zahlreiche weitere Bemerkungen zu op. 81 in Dvořák-Literatur, allg. Literatur zur Kammermusik (einschl. Konzertführern) und allg. Literatur zur Musikgeschichte des 19. Jahrhunderts.

319 Vgl. Fabian Huss: The Chamber Music of Frank Bridge [Diss.], University of Bristol: Bristol 2010, https://core.ac.uk/download/pdf/33134389.pdf (insb. S. 161-182); Trevor Bray: Frank Bridge. A Life in Brief, 2016, http://trevor-braymusic-research.co.uk/Bridge\%20LinB/contents.html; Fabian Huss: The Music of Frank Bridge, The Boydell Press: Woodbridge 2015.

Die frühe, noch tonale Kammermusik von Bridge zwischen Jahrhundertwende und Weltkriegsende steht ganz im Zentrum seines damaligen Schaffens. Sie ist voller solcher Momente wie der skizzierten Passage des Klavierquintetts, über die man sich an dieser Stelle mit gleichem Recht beugen könnte. Die extrovertierte Rückkehr des Kopfthemas im I. Satz der Cellosonate d-Moll H. 125 (1913-17) etwa. Oder die strahlende, vollstimmige Eröffnung des Streichsextetts Es-Dur H. 107 (1906-12). Die schmeichelnden Vorhaltgesten in der Mitte des II. Satzes des Klavierquartetts fis-Moll H. 94 (1910). Das düstere Kopfthema des I. Satzes des Klaviertrios c-Moll H. 79 (1907). Die nebeligen Akkordflächen des Beginns des Finales des Streichquartetts Nr. 2 g-Moll H. 115 (1914-15). Der Beginn des Andante aus dem Streichquintett e-Moll H. 7 (1901), der klingt, als würde der späte Dvořák der Neunten Sinfonie e-Moll op. 95 Aus der neuen Welt Urlaub in Cambridgeshire machen. Der zweifach kurz vor dem abschließenden Agitato der Stretta am Ende des I. Satzes des Streichquartetts Nr. 1 e-Moll H. 70 (1906) auftauchende, lang gehaltene, terzlose dominant Sept-Non-Akkorde, der in seinem scharf akzentuierten musikalischen Kontext faszinierend isoliert klingt.

320 Vgl. Catherine Lorent: Florent Schmitt, Bleu Nuit: Paris 2012.

321 Vgl. auch 1989, Live Broadcast via BBC Radio 3 (https://soundcloud.com/us er-272563906/schmitt-piano-quintet-in-b-minor-op-51-1-lent-et-grave-animewith-57-sec-spoken-introduction-2-lent; https://soundcloud.com/user-2725639 06/schmitt-piano-quintet-in-b-minor-op-51-movement-3 - Music Group of London); 1993, EMI Classics 077775484021 (Florent Schmitt, Quatuor Clavet - Reissue [II. Satz], Aufnahme von 1935); 2008, Timpani 1C1152 (Chris- 
tian Ivaldi, Quatuor Stanislas); 2011, Live at Hortus Festival/Niederlande (https://www.concertzender.nl/programma/concertzender-live-4135/ - Hortus Ensemble); 2011, Naxos 8.570489 (Solisten-Ensemble Berlin).

322 Vgl. Anna Zassimova: Georges Catoire. Seine Musik, sein Leben, seine Ausstrahlung, Kuhn: Berlin 2011, S. 323-328.

323 Vgl. Allan Evans: Ignaz Friedman. Romantic Master Pianist, Indiana University Press: Bloomington/IN 2009.

324 Vgl. Lewis Foreman: Bax. A Composer and His Times, 3. Auflg., The Boydell Press: Woodbridge 2007.

325 Vgl. auch 2010, Naxos 8.572474 (Ashley Wass, The Tippett Quartet); 2010, Live at Indian Summer in Levoca Festival [Slowakei] (https://www.youtube. com/watch?v=M-jhPsZvxhA - Jonathan Powell, Post Scriptum Quartet).

326 Vgl. Jean-Michael Nectoux: Gabriel Fauré. A Musical Life, Cambridge University Press: Cambridge 1991; Claudia Breitfeld: Form und Struktur in der Kammermusik von Gabriel Fauré, Bärenreiter: Kassel 1992; Marie-Maud Thomas: »Stilelemente in Faurés Kammermusik«, in: Gabriel Fauré. Werk und Rezeption, hrsg. von Peter Jost, Bärenreiter: Kassel 1996, S. 58-65.

327 Dieser Paragraph übernimmt Auszüge aus Frédéric Döhl: »Postmoderne, biographische und gattungsgeschichtliche Motive in Alfred Schnittkes Klavierquintett«, in: Postmoderne hinter dem Eisernen Vorhang, hrsg. von Stefan Weiss/Amrei Flechsig, G. Olms: Hildesheim 2013, S. 107-119. Vgl. des Weiteren Joachim Hansberger: »Alfred Schnittke im Gespräch über sein Klavierquintett und andere Kompositionen«, in: Zeitschrift für Musikpädagogik 20/7 (1982), S. 44-50; Alfred Schnittke: »In memoriam (Orchesterfassung des Klavierquintetts)« und »Klavierquintett«, in: Alfred Schnittke zum 60. Geburtstag, hrsg. von den Internationalen Musikverlagen Hans Sikorski, Internationale Musikverlage Hans Sikorski: Hamburg 1994, S. 105-106 und 119-120; Gottfried Eberle: »Figur und Struktur von Kreuz und Kreis am Beispiel von Alfred Schnittkes Klavierquintett«, in: Alfred Schnittke zum 60. Geburtstag, hrsg. von den Internationalen Musikverlagen Hans Sikorski, Internationale Musikverlage Hans Sikorski: Hamburg 1994, S. 46-54; Frans C. Lemaire: »Tod einer Mutter - zum Klavierquintett von Alfred Schnittke«, in: Alfred Schnittke zum 60. Geburtstag, hrsg. von den Internationalen Musikverlagen Hans Sikorski, Internationale Musikverlage Hans Sikorski: Hamburg 1994, S. 223-226; Basil Smallman: The Piano Quartet and Quintet. Style, Structure, and Scoring, Clarendon Press: Oxford 1996, S. 150-157; Alexander Ivashkin: Alfred Schnittke, Phaidon: London 1996; Alexander Ivashkin (Hrsg.): A Schnittke Reader, Indiana 
University Press: Bloomington/IN 2002, S. 87-90; Walter-Wolfgang Sparrer: »Quintett für Klavier und Streicher (1972)«, in: Geschichte der Musik im 20. Jahrhundert: 1945-1975, hrsg. von Hanns-Werner Heister, Laaber: Laaber, 2005, S. 303-307; Dorothea Redepenning: »Polystilistik in Alfred Schnittkes Konzeption«, in: dies., Geschichte der russischen und sowjetischen Musik, Bd. II/2, Laaber: Laaber 2008, S. 684-699; Christian Storch: »Tanz an die verstorbene Mutter? - Der B-A-C-H-Walzer in Alfred Schnittkes Klavierquintett«, in: L'art macabre, hrsg. von Uli Wunderlich, Europäische Totentanzvereinigung: Düsseldorf 2008, S. 201-212.

328 Vgl. Christopher Fox: »Tempestuous Times: The Recent Music of Thomas Adès«, in: The Music Times 145 (2004), S. 41-56; John Roeder: »Co-operating Continuities in the Music of Thomas Adès«, in: Music Analysis 25/1-2 (2006), S. 121-154; Thomas Adès/Tom Service: Thomas Adès: Full of Noises. Conversations with Tom Service, Farrar, Straus and Giroux: New York 2012; Dominic Wells: »Plural Styles, Personal Style: The Music of Thomas Adès«, in: Tempo 66/260 (2012), S. 2-14; Emma Gallon: »Narrativities in the Music of Thomas Adès: The Piano Quintet and 〉Brahms««, in: Music and Narrative since 1900, hrsg. von Michael L. Klein/Nicholas Reyland, Indiana University Press: Bloomington/IN 2013, S. 216-233; Philip Stoecker: »Aligned Cycles in Thomas Adès's Piano Quintet«, in: Music Analysis 33/1 (2014), S. 32-64; Edward Venn: »Thomas Adès and the Spectres of `Brahms««, in: Journal of the Royal Musical Association 140/1 (2015), S. 163-212; Felix Wörner: »Tonality as >Irrationally Functional Harmony«: Thomas Adès’s Piano Quintet«, in: Tonality since 195, hrsg. von Felix Wörner/Ullrich Scheideler/Philip Rupprecht, Franz Steiner Verlag: Stuttgart 2017, S. 295-312.

329 Vgl. http://thomasades.com/compositions/piano_quintet.

330 Vgl. Fernando J. Cabañas Alamán: Antón García Abril. Sonidos en libertad, 2. Auflg., Ediciones del ICCMU: Madrid 2001; Paula Coronas Valle: Antón García Abril. Imagen y música en armonía, Primera Edición: Málaga: 2009; Paula Coronas Valle: La obra pianistica de Antón García Abril, Universidad de Málaga: Málaga 2010; Yanira Soria: Antón García Abril: Alba de los Caminos and the Concept of Polimelodiosidad [Diss.], University of Houston: Houston 2016, https://uh-ir.tdl.org/handle/10657/2643, insb. S. 16-36.

331 Vgl. Nate Chinen: Playing Changes. Jazz for the New Century, Pantheon: New York 2018, S. 137-155. 
332 Vgl. etwa die Projektübersicht für >Klassische Werkeく bei Iyers Mainzer Verleger Schott, https://www.eamdc.com/audio/presskit/83b47b02-56a1-11e7-ad dd-0a1edf4187b9/.

333 Vgl. Nate Chinen: »DNA of a Polymath, Restlessly Mutating«, in: The New York Times (7. März 2014), https://www.nytimes.com/2014/03/09/arts/music/v ijay-iyers-new-release-bridges-string-quartet-and-improvisation.html.

334 Vgl. 2014, ECM 2372 (Vijay Iyer, Miranda Cuckson, Michi Wiancko, Kyle Armbrust, Kivie Cahn-Lipman).

335 An deren Projekt Fragments hatte Iyer sich neben Komponistinnen und Komponisten wie Charles Wuorinen, Sofia Gubaidulina, John Harbison, Bruce Adolphe und Stephen Hartke beteiligt. Die Idee des Ensembles war, ausgehend von eigenen Erfahrungen mit einem Schubert-Fragment, zeitgenössische Komponisten zu bitten, ausgehend von weiteren Fragmenten bekannter Komponisten etwas zu schreiben. Iyer entschied sich für Mozart. So entstand 2011 Mozart Effects. Vgl. https://www.brentanoquartet.com/project/an-introductionto-the-brentano-string-quartets-fragments-project/.

$336 \mathrm{Vgl}$. https://www.youtube.com/watch?time_continue=2\&v=-hOFmDcT_qc.

337 Vgl. Zachary Woolfe: »Hear the Piece That Won One of Music's Biggest Prizes«, in: The New York Times (28.11.2017), https://www.nytimes.com/20 17/11/28/arts/music/grawemeyer-award-bent-sorensen.html. Anlass für Woolfes Kommentar war die Verkündung des Gewinns des Grawemeyer Kompositionspreises 2018 für sein Triplekonzert für Klaviertrio und Orchester L'isola della città. Mit 100.000 Dollar dotiert, eine der bedeutendsten Auszeichnungen ihrer Art.

338 Evoziert Papillons offenkundig die Erinnerung an Robert Schumanns gleichnamiges op. 2, verweist Rosenbad auf die letzte Erzählung von Karen Blixen, Ehrengard, die zur Zeit von Schumanns Schaffen spielt und dort einem Schloss seinen Namen gibt.

339 Vgl. https://de.schott-music.com/shop/autoren/fazil-say; https://www.youtube. com/watch?v=RV8LIDQz_Tw.

340 Vgl. 2019, Warner 0190295504656.

341 Vgl. 2018, Live in Brüssel, https://www.youtube.com/watch?v=P1hA0Rz4 QFo; 2018, Live in Melbourne, https:/www.youtube.com/watch?v=jB5nMI4 U90s. Noten: Mainz: Schott o.J..

342 Vgl. 1999, Philips 4467102 (Peter Serkin, Guarneri Quartet - Uraufführungsbesetzung). Noten: Mainz: Schott 1993. 
343 »The work is in one movement of many changing characters and contrasts. The moods and materials of the piano are contrasted with those of the string quartet, which, itself, is a combination of four different strands that maintain somewhat independent existences, played by the four strings. « Elliott Carter: Programmhinweis, https://www.boosey.com/pages/cr/catalogue/cat_detail?=\& musicid=3066\&langid $=1$.

344 Vgl. 2003, Mode 128 (Ursula Oppens, Arditti Quartet - Uraufführungsbesetzung, Ersteinspielung); 2001, Live [o.A.] (https://www.youtube.com/watch ?v=PvhUqpOcxZE - Marc-André Hamelin, Arditti Quartet). Noten: London: Boosey \& Hawkes 1997. 


\section{Musikwissenschaft}

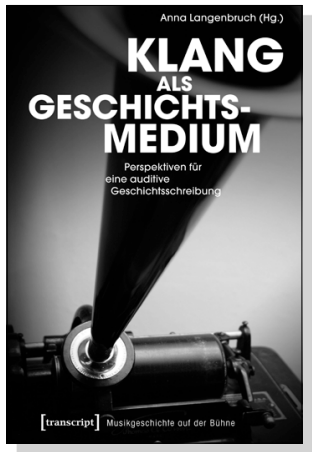

Anna Langenbruch (Hg.)

Klang als Geschichtsmedium

Perspektiven für eine auditive Geschichtsschreibung

Januar 2019, 282 S., kart., Klebebindung, 19 SW-Abbildungen $34,99 €(D E), 978-3-8376-4498-2$

E-Book: 34,99 € (DE), ISBN 978-3-8394-4498-6

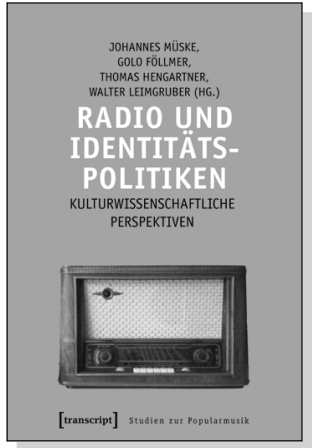

Johannes Müske, Golo Föllmer,

Thomas Hengartner, Walter Leimgruber (Hg.)

\section{Radio und Identitätspolitiken}

Kulturwissenschaftliche Perspektiven

Januar 2019, 290 S., kart., Klebebindung, 22 SW-Abbildungen $34,99 €(D E), 978-3-8376-4057-1$

E-Book: 34,99€ (DE), ISBN 978-3-8394-4057-5

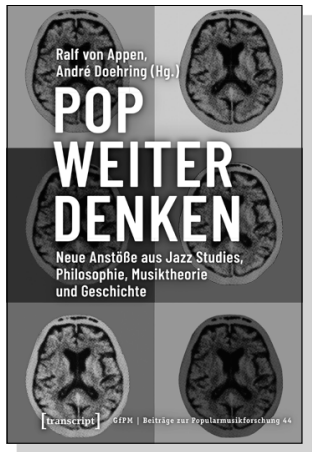

Ralf von Appen, André Doehring (Hg.)

\section{Pop weiter denken}

Neue Anstöße aus Jazz Studies, Philosophie, Musiktheorie und Geschichte

2018, 268 S., kart., Klebebindung, 6 Farbabbildungen $22,99 €(D E), 978-3-8376-4664-1$

E-Book: 20,99 € (DE), ISBN 978-3-8394-4664-5 


\section{Musikwissenschaft}
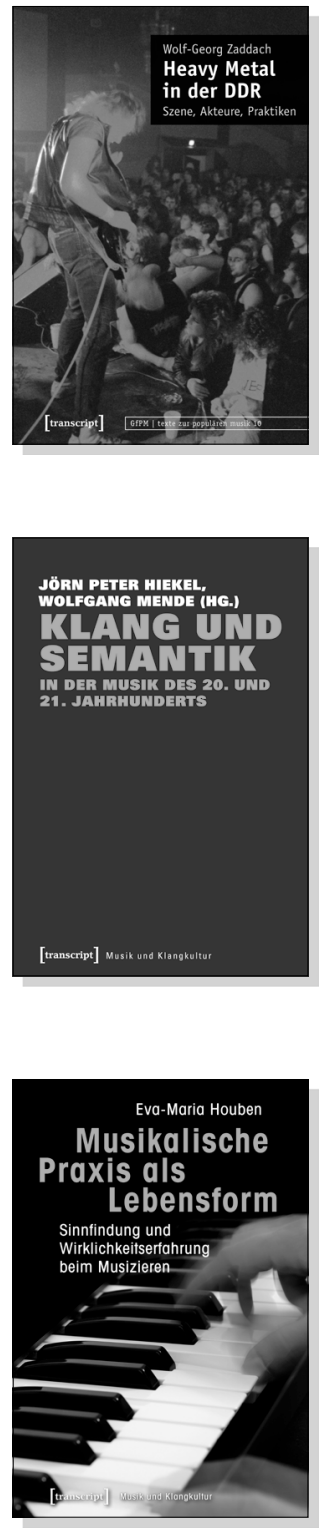

Wolf-Georg Zaddach

Heavy Metal in der DDR

Szene, Akteure, Praktiken

2018, 372 S., kart., Klebebindung,

21 SW-Abbildungen, 11 Farbabbildungen

39,99€ (DE), 978-3-8376-4430-2

E-Book: 39,99 € (DE), ISBN 978-3-8394-4430-6

Jörn Peter Hiekel, Wolfgang Mende (Hg.)

Klang und Semantik in der Musik

des 20. und 21. Jahrhunderts

2018, 268 S., kart., Klebebindung, 42 SW-Abbildungen

$34,99 €(D E), 978-3-8376-3522-5$

E-Book: 34,99 € (DE), ISBN 978-3-8394-3522-9

Eva-Maria Houben

Musikalische Praxis als Lebensform

Sinnfindung und Wirklichkeitserfahrung beim Musizieren

2018, 246 S., kart., Klebebindung, 84 SW-Abbildungen

29,99€ (DE), 978-3-8376-4199-8

E-Book: kostenlos erhältlich als Open-Access-Publikation 